\title{
Evaluation of the Transport and Resuspension of a Simulated Nuclear Waste Slurry
}

T. E. Carleson

D. C. Brown

R. E. Hart

M. E. Peterson, Technical Manager

September 1987

Prepared for the U.S. Department of Energy under Contract DE-AC06-76RLO 1830

Pacific Northwest Laboratory Operated for the U.S. Department of Energy by Battelle Memorial Institute 


\title{
DISCLAIMER
}

This report was prepared as an account of work sponsored by an agency of the United States Government. Neither the United States Government nor any agency thereof, nor Battelle Memorial Institute, nor any of thesir employees, makes any wartanty, expressed or implied, or assumes any legal liability or responsibility for the accuracy, completeness, or usefulness of any information, apparatus, product. or process disclosed, or represents that its use would not infringe privately owned rights. Reference herein to any specific commercial product, process, or service by trade name, trademark, manufacturer, or otherwise, does not necessarily constitute or imply its endorsement, recommendation, or favoring by the United States Government of any agency thereof, or Battelle Memorial institute. The views and opinions of asthors expressed herein do not necessarly state or reflect those of the United States Government or any agency thereof, or Batteile Memorial Institute.

\author{
PACIFIC NORTHWEST LABORATORY \\ operated by \\ BATTELLE MEMORIAL INSTITUTE \\ for the \\ UNITED STATES DEPARTMENT OF ENERGY \\ under Contract DE-AC06-76RLO 1830
}

Printed in the United States of America
Availabie from
Nationat Technical Information Service
United States Department of Commerce
5285 Port Royal Rosd
Springfield, Virginia 22161
NTIS Price Codes
Microfiche A01
Printed Copy
Pages
$001-025$
$026-050$




\title{
33679000442832
}

\author{
NUCLEAR WASTE TREATMENT PROGRAM
}

EVALUATION OF THE TRANSPORT AND RESUSPENSION OF A SIMULATED NUCLEAR WASTE SLURRY

T. E. Carleson(a)

D. C. Drown (a)

R. E. Hart (a)

M. E. Peterson, Technical Manager

September 1987

Prepared for

U.S. Department of Energy under Contract DE-ACO6-76RLO 1830

Pacific Northwest Laboratory Richland, Washington 99352

(a) Department of Chemical Engineering University of Idaho Moscow, Idaho 83843 
$\underline{\text { SUMMARY }}$

The Department of Chemical Engineering at the University of Idaho conducted research on the transport and resuspension of a simulated high-level nuclear waste slurry under a grant through the Northwest College and University Association for Science (NORCUS). This research is being sponsored by the Nuclear Waste Treatment Program (NWTP). at the Pacific Northwest Laboratory (PNL). (a) The NWTP conducts generic research aimed toward the future commercialization of high-level liquid waste (HLLW) treatment technologies. The NWTP also addresses the research and development needs for the HLLW treatment demonstration being conducted for the Department of Energy (DOE) at west Valley, New York.

In the United States, the reference process for treating both defense and civilian HLLW is vitrification using the liquid-fed ceramic melter process. In this process, the HLLW is blended with glass-forming materials, and the resulting slurry is metered into a refractory-lined, joule-heated melting tank. The melter feed pump, as well as other process pumps, must be able to transport the slurry at velocities high enough to prevent the deposition of solids in the equipment. Also, the pump must be sufficiently over-sized to be able to resuspend any solids that might sett?e out during unplanned pump outages. The nonNewtonian behavior of the slurry complicates the evaluation of the transport and resuspension characteristics of the slurry.

The resuspension of a simulated (nonradioactive) melter feed slurry was evaluated using a siurry designated as WV-205. The simulated slurry was developed for the West Valley Demonstration Project and was used during a pilot-scale ceramic melter (PSCM) experiment conducted at PNL in July 1985 (PSCM-21).

The scope of this study involved determining the transport characteristics of a fully suspended slurry and the resuspension characteristics of settled solids in a pilot-scale pipe loop. The goal of the work was to predict the

(a) Operated for the U.S. Department of Energy by Battelle Memorial Institute under Contract DE-AC06-76RLO 1830. 
transport and resuspension of a full-scale system based on rheological data for a specific slurry. The rheological behavior of the slurry was evaluated using a concentric cylinder rotational viscometer, a capillary tube viscometer, and the pilot-scale pipe loop. The results obtained from the three approaches were compared.

The slurry contained 36 wt\% total solids with 25 wt\% suspended solids. The slurry density was $1.3 \mathrm{~g} / \mathrm{mL}$. The slurry contained solids with a median volume diameter of 5 to $9 \mathrm{\mu m}$. The fine particle size resulted in a homogeneous slurry with a slow settling rate of $0.0012 \mathrm{~cm} / \mathrm{min}$ at $24^{\circ} \mathrm{C}$.

The rheological properties of the slurry permitted it to be classified as a yield pseudoplastic fluid or, in some cases, a Bingham plastic fluid. The shear stress versus shear rate data from the concentric cylinder rotational viscometer were evaluated using both a linear regression method and a nonlinear curve fit method. The rheological parameters varied depending on the method chosen. The nonlinear curve fit was found to produce the best statistical answer for the yield pseudoplastic model. Wall slip was evaluated and was not occurring in this viscometer.

The rheological parameters were also determined using a capillary tube viscometer. The data were fit to the yield pseudoplastic model using a nonlinear curve fit method. The shear rate data were corrected to account for the non-Newtonian fluid behavior prior to the nonlinear regression analysis. Wall slip was evaluated and was not occurring in this viscometer.

A pilot-scale pipe loop was used to evaluate the transport and resuspension of the simulated melter feed slurry. The pipe loop contained pressure transducers interfaced with a computer data acquisition system to measure and record the transient and steady-state pressure drops for both the slurry transport and the resuspension experiments. The system was calibrated with both water and a sugar solution. The pilot-scale system used three different pipe diameters, $6.35 \mathrm{~mm}, 9.52 \mathrm{~mm}$, and $12.7 \mathrm{~mm}$. The use of three pipe diameters permits scale-up of the data to other pipe diameters.

The rheological behavior of the slurry was determined from the pipe 100p experiments. The rheological parameters differ from the results obtained using 
the rotational viscometer but are in better agreement with results from the capillary tube viscometer. This can be explained by the different shear rates evaluated in the systems. The maximum shear rates evaluated in the concentric cylinder rotational viscometer were a factor of four smaller than the shear rates evaluated in the pipe loop. The capillary tube viscometer and pipe loop were evaluated over the same shear rates. The rheological models developed for the rotational viscometer, capillary viscometer, and pipe loop were used to plot the shear stress versus shear rate over a range of 0 to $2000 \mathrm{~s}^{-1}$. All three methods were in agreement at the low shear rates.

For the pipe loop experiments, the laminar-to-turbulent transition point for the siurry ranged from a Reynolds number of approximately 2800 with the 6.35-mm-diameter pipe to 3600 with the 12.7-mm-diameter pipe. The transition Reynolds numbers and the laminar data (friction factor versus Reynolds number relationships) agree with predictions using Hanks' model (Hanks and Hanks 1984) for a yield pseudoplastic fluid. An empirical parameter in the yield pseudoplastic model must be corrected in order to correlate the turbulent data (friction factor versus Reynolds number relationships).

The resuspension experiments were conducted after the slurry had settled in the pipe loop for $20 \mathrm{~h}$ and $44 \mathrm{~h}$. After these settling times, the slurry was relatively easy to resuspend. Resuspension occurred at flow rates of 10 to $30 \mathrm{~mL} / \mathrm{s}$, which corresponded to Reynolds numbers in the laminar region. Typically, resuspension would not occur until turbulent flow was reached. The WV-205 slurry contained small particles which created a homogeneous slurry. The lift and drag forces on the particles easily overcame the gravity forces, which enabled resuspension to occur at the low flowrates and in the laminar region. Therefore, designing the pumping system to transport the slurry above the laminar and transition regions will permit the resuspension of a settled bed of WV-205 slurry. 


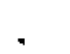

'

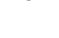




\section{CONTENTS}

SUMMARY

i $i$ i

1.0 INTRODUCTION AND OBJECTIVES $\ldots \ldots \ldots \ldots \ldots \ldots \ldots \ldots \ldots \ldots \ldots \ldots \ldots \ldots \ldots \ldots \ldots \ldots$

2.0 CONCLUSIONS AND RECOMMENDATIONS $\ldots \ldots \ldots \ldots \ldots \ldots \ldots \ldots \ldots \ldots \ldots \ldots \ldots \ldots . \ldots \ldots$

3.0 TRANSPORT OF NON-NEWTONIAN FLUIDS $\ldots \ldots \ldots \ldots \ldots \ldots \ldots \ldots \ldots \ldots \ldots \ldots \ldots \ldots \ldots$

3.1 BACKGROUND FOR ROTATIONAL VISCOMETER EXPERIMENTS .......... 3.3

3.2 BACKGROUND FOR CAPILLARY AND PIPE LOOP EXPERIMENTS $\ldots \ldots \ldots \ldots . .$.

4.0 RESUSPENSION OF NON-NEWTONIAN SLURRIES $\ldots \ldots \ldots \ldots \ldots \ldots \ldots \ldots \ldots \ldots \ldots \ldots$

5.0 SIMULATED MELTER FEEd SLURRY PREPARATION AND CHARACTERIZATION $\ldots \ldots .5 .1$

5.1 MELTER FEED SLURRY PREPARATION $\ldots \ldots \ldots \ldots \ldots \ldots \ldots \ldots \ldots \ldots \ldots \ldots \ldots . \ldots \ldots \ldots \ldots \ldots \ldots$

5.1 .1 Composition of WV-205 Slurry .................. 5.2

5.1.2 Slurry Preparation Procedures ................... 5... 3

5.2 MELTER FEED SLURRY CHARACTERIZATION $\ldots \ldots \ldots \ldots \ldots \ldots \ldots \ldots \ldots \ldots \ldots . \ldots$

5.2 .1 Physical Properties ........................ 5.5

5.2 .2 Rheological Properties ...................... 5.11

6.0 PILOT-SCALE TRANSPORT OF HOMOGENEOUS SLURRY $\ldots \ldots \ldots \ldots \ldots \ldots \ldots \ldots \ldots . . \ldots . \ldots$

6.1 DESCRIPTION OF EXPERIMENTAL APPARATUS $\ldots \ldots \ldots \ldots \ldots \ldots \ldots \ldots \ldots . . \ldots \ldots$

6.1 .1 Capillary Viscometer .......................... 6.1

6.1 .2 Pipe Loop Apparatus ......................... 6.3

6.2 CALIBRATION OF APPARATUS $\ldots \ldots \ldots \ldots \ldots \ldots \ldots \ldots \ldots \ldots \ldots \ldots \ldots \ldots \ldots \ldots \ldots \ldots \ldots \ldots \ldots \ldots$

6.3 NEWTONIAN FLUID EXPERIMENTS $\ldots \ldots \ldots \ldots \ldots \ldots \ldots \ldots \ldots \ldots \ldots \ldots \ldots \ldots . \ldots . \ldots \ldots$

6.4 SLURRY TRANSPORT EXPERIMENTS $\ldots \ldots \ldots \ldots \ldots \ldots \ldots \ldots \ldots \ldots \ldots \ldots \ldots \ldots . \ldots \ldots$

6.4 .1 Capillary Tube Results ........................ 6.11

6.4 .2 Pipe Loop Results .......................... 6.12

6.4 .3 Laminar Flow Results ......................... 6.15 
6.4 .4 Laminar and Turbulent Results ................ 6.20

7.0 RESUSPENSION OF SETTLED SLURRY $\ldots \ldots \ldots \ldots \ldots \ldots \ldots \ldots \ldots \ldots \ldots \ldots \ldots \ldots$

7.1 OESCRIPTION OF RESUSPENSION APPARATUS $\ldots \ldots \ldots \ldots \ldots \ldots \ldots \ldots \ldots . . \ldots$

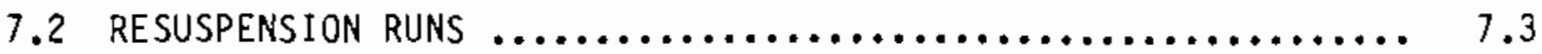

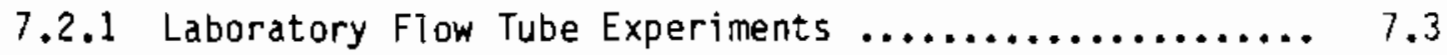

7.2.2 Pipe Loop Resuspension Experiments ............. 7.4

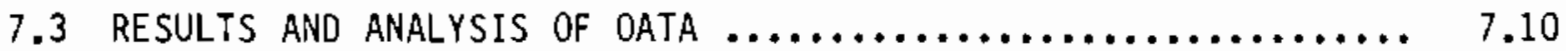

8.0 COMPARISON OF RESULTS TO OTHER STUOIES $\ldots \ldots \ldots \ldots \ldots \ldots \ldots \ldots \ldots \ldots \ldots . . \ldots . \ldots$

8.1 HOMOGENEOUS SLURRY TRANSPORT EXPERIMENTS $\ldots \ldots \ldots \ldots \ldots \ldots \ldots \ldots . . . . . .$.

8.2 SLURRY RESUSPENSION EXPERIMENTS $\ldots \ldots \ldots \ldots \ldots \ldots \ldots \ldots \ldots \ldots \ldots . \ldots .4$

9.0 COMPARISON OF LABORATORY TO PILOT SCALE RESULTS $\ldots \ldots \ldots \ldots \ldots \ldots \ldots . . . . . . . . .$.

10.0 RAMIFICATIONS OF RESULTS TO DESIGN OF FULL-SCALE SYSTEMS $\ldots \ldots \ldots 10.1$

10.1 PUMPING REQUIREMENTS FOR TRANSPORT $\ldots \ldots \ldots \ldots \ldots \ldots \ldots \ldots \ldots \ldots$

10.2 PUMPING REQUIREMENTS FOR RESUSPENSION $\ldots \ldots \ldots \ldots \ldots \ldots \ldots \ldots \ldots . \ldots$

11.0 REFERENCES $\ldots \ldots \ldots \ldots \ldots \ldots \ldots \ldots \ldots \ldots \ldots \ldots \ldots \ldots \ldots \ldots \ldots \ldots \ldots \ldots \ldots$ 
FIGURES

1.1 Vitrification Process $\ldots \ldots \ldots \ldots \ldots \ldots \ldots \ldots \ldots \ldots \ldots \ldots \ldots, 1.2$

4.1 Shields Diagram for Particulate Transport .................... 4.4

4.2 Generalized Phase Diagram for Transport of Suspensions ......... 4.5

4.3 Mean Particle Diameter Versus Mean Velocity for Identification of Heterogeneous and Homogeneous Flow Patterns $\ldots \ldots \ldots \ldots \ldots \ldots . .4 .7$

4.4 Friction Loss Versus Velocity for Determining Critical

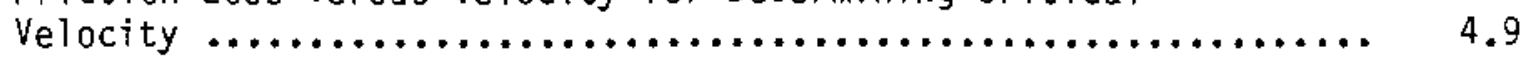

5.1 Setting Rate Curves for WV-205 Slurry at Three Total Solid

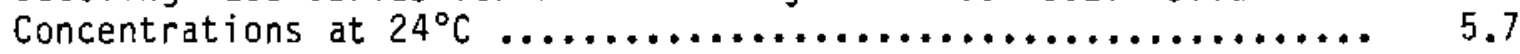

5.2 Settling Rate Curves for WV-205 Slurry at Three Total Solid

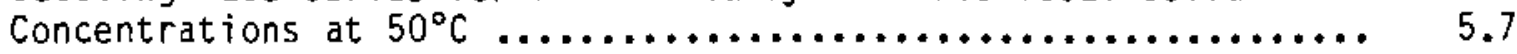

5.3 Settling Rate Determined by Measuring the Slurry Interface Height Versus Time in a Vertical Graduated Cylinder ............ 5.9

5.4 Settling Rate Determined by Measuring the Slurry Interface Height Versus Time in a Horizontal Cylinder ................. 5.10

5.5 Schematic of a Haake Viscometer $\ldots \ldots \ldots \ldots \ldots \ldots \ldots \ldots \ldots \ldots \ldots . \ldots .12$

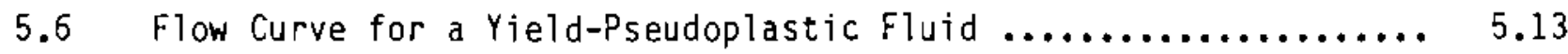

5.7 Shear Stress Versus Shear Rate for the West Valley Slurry Using

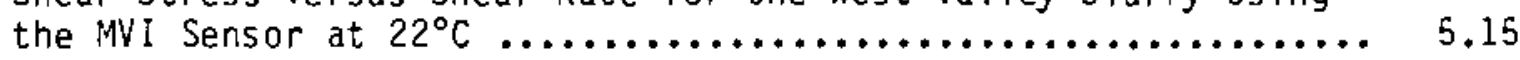

5.8 Shear Stress Versus Shear Rate for the West Valley Slurry

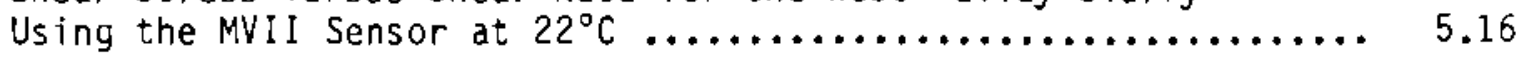

5.9 Angular Speed Versus Rotational Cylinder Stress for the WV-205 Slurry Using Three Different Sensors $\ldots \ldots \ldots \ldots \ldots \ldots \ldots . . .6 .18$

5.10 Angular Speed Versus the Ratio of Cup Radius to Bob Radius at Various Rotational Cylinder Stresses ....................... 5.19

5.11 Shear Stress Versus Shear Rate for West Valley Slurry Indicating Differences Between Linear and Nonlinear Curve Fitting Techniques

5.12 Rheograms for 10,36 , and 50 wt $\%$ Solids Concentration Slurry at $22^{\circ} \mathrm{C}$ 
5.13 Rheograms for 10, 36, and $50 \mathrm{wt} \%$ Solids Concentration Slurry at $50^{\circ} \mathrm{C}$

5.14 Bob Stress Versus Angular Speed for WV-205 Slurry Using

Three Different Sensors

5.15 Bob Stress Versus Shear Rate for WV-205 Slurry Using

Three Different Sensors

5.16 Apparent Viscosity Versus Shear Rate for 10 wt\% and

36 wt\% Solids Concentration Slurry at $22^{\circ} \mathrm{C}$ and $50^{\circ} \mathrm{C}$

5.17 Apparent Viscosity Versus Shear Rate for 50 wt\% Solids

Concentration Slurry at $22^{\circ} \mathrm{C}$ and $50^{\circ} \mathrm{C}$

6.1 Schematic Diagram of Capillary Viscometer ................. 6.2

6.2 Schematic Diagrarn of Pipe Flow Test Loop $\ldots \ldots \ldots \ldots \ldots \ldots \ldots . \ldots . . . . .6$

6.3 Shear Stress Versus Shear Rate for a 42 wt: Sugar Solution Using the Capillary Tube Viscometer with All Four Tube Diameter.

6.4 Pipe-Loop Calibrations of Shear Stress Versus Shear Rate Using a $60 \mathrm{wt} \%$ Sugar-Water Solution in the $6.35-\mathrm{mm}-1 \mathrm{D}$ Pipe ......

6.5 Logarithmic Plot of Pipe Loop Calibrations Showing Shear Stress

Versus Shear Rate Using Water in the 6.35-mm-10 Pjpe

6.6 Fanning Friction Factor Versus Reynolds Number for All Three Pipe Loop Diameters Using Both Water and Sugar-Water Solution ...

6.7 Logarithmic Plot of Shear Stress Versus Adjusted Shear Rate for the WV-205 Slurry Determined Using the Capillary Tube Viscometer

6.8 Shear Stress Versus Shear Rate for WV-205 Slurry Determined

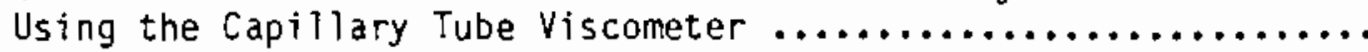

6.9 Evaluation of Wall Slip in the Capillary Tube Viscometer

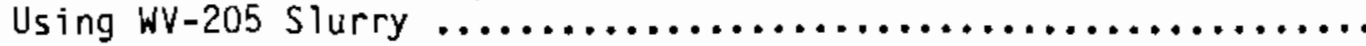

6.10 Logarithmic Plot of Shear Stress Versus Shear Rate Initial

Results for WV-205 Slurry in Laminar Flow in Three Pipe

Diameters

6.11 Initial Shear Stress Versus Shear Rate Results for the WV-205 slurry

6.12 Initial Shear Stress Versus Shear Rate Results for the 
6.13 Logarithmic Plot of Shear Stress Versus Shear Rate for the W-205 Slurry in Laminar Flow in Three Pipe Diameters .......... 6.19

6.14 Shear Stress Versus Shear Rate in Replicate Runs for the WV-205 Slurry Fit to the Bingham Plastic Model ............... 6.21

6.15 Shear Stress Versus Shear Rate in Replicate Runs for the WV-205 Slurry Fit to the Yield Pseudoplastic Model ........... 6.22

6.16 Shear Stress Versus Shear Rate for WV-205 Slurry for the

Two Testing Periods

6.17 Logarithmic Plot of Shear Stress Versus Shear Rate for WV-205 Slurry Using 6.35-mm-ID Pipe for the Two Testing Periods

6.18 Logarithmic Plot of Shear Stress Versus Shear Rate for WV-205 Slurry Using 9.52-mm-ID Pipe for the Two Testing Periods

6.19 Logarithmic Plot of Shear Stress Versus Shear Rate for WV-205 Slurry Using 12.7-mm-ID Pipe for the Two Testing Periods

6.20 Logarithmic Plot of Shear Stress Versus Shear Rate for WV-205 Slurry in Laminar and Turbulent Flow Using Three Pipe Diameters

6.21 Fanning Friction Factor Versus Reynolds Number for WV-205

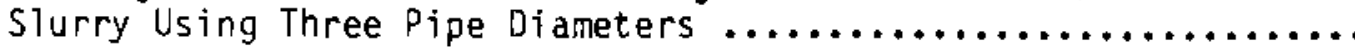

7.1 Resuspension Apparatus of 24-in.-Long Glass Tube

7.2 Pressure Drop Versus Resuspension Time for WV-205 Slurry at a $15.7 \mathrm{~mL} / \mathrm{s}$ Flow Rate After $2 \mathrm{U} h$ of Settling in 12.7-mm-ID Pipe

7.3 Pressure Drop Versus Time for Fully Homogeneous Slurry Transported Through 12.7-mm-ID Pipe

7.4 Pressure Drop Versus Resuspension Time for WV-205 Siurry at a $26.4 \mathrm{~mL} / \mathrm{s}$ Flow Rate After $20 \mathrm{~h}$ of Settling in 12.7-mrn-ID Pipe

7.5 Pressure Drop Versus Resuspension Time for WV-205 Slurry at a $39.5 \mathrm{~mL} / \mathrm{s}$ Flow Rate After $20 \mathrm{~h}$ of Settling in 12.7-mm-ID Pipe

7.6 Pressure Drop Versus Resuspension Time for WV-205 Siurry at a $114 \mathrm{~mL} / \mathrm{s} \mathrm{Flow} \mathrm{Rate} \mathrm{After} 20 \mathrm{~h}$ of Settling in 12.7-min-ID Pipe 
7.7 Pressure Drop Versus Resuspension Time for WV-205 Slurry at a $194 \mathrm{~mL} / \mathrm{s}$ Flow Rate After $20 \mathrm{~h}$ of Settling in

12.7-mm-1D Pipe

7.8 Pressure Drop Versus Resuspension Time for WV-205 Slurry at a $0.29 \mathrm{~mL} / \mathrm{s}$ Flow Rate After $44 \mathrm{~h}$ of Settling in 12.7-mm-ID Pipe

7.9 Pressure Drop Versus Resuspension Time for WV-205 Slurry at a $40 \mathrm{~mL} / \mathrm{s}$ Flow Rate After $44 \mathrm{~h}$ of Settiting in 12.7-mm-1D Pipe

7.10 Pressure Drop Versus Resuspension Time for WV-205 Slurry at a $9.4 \mathrm{~mL} / \mathrm{s}$ Flow Rate After $20 \mathrm{~h}$ of Settling in

9.52-mm-1D Pipe

7.11 Pressure Drop Versus Resuspension Time for WV-205 Slurry at a $51.5 \mathrm{~mL} / \mathrm{s}$ Flow Rate After $20 \mathrm{~h}$ of Settling in 9.52-mm-ID Pipe

7.12 Pressure Drop Versus Resuspension Time for WV-205 Slurry at a $73.8 \mathrm{~mL} / \mathrm{s}$ Flow Rate After $20 \mathrm{~h}$ of Settling in 9.52-mm-ID Pipe

7.13 Pressure Drop Versus Resuspension Time for WV-205 Slurry at a $117 \mathrm{~mL} / \mathrm{s}$ Flow Rate After $20 \mathrm{~h}$ of Settling in 9.52-mm-ID Pipe

7.14 Pressure Drop Versus Resuspension Time for WV-205 SIurry at a $50.1 \mathrm{~mL} / \mathrm{s}$ Flow Rate After $44 \mathrm{~h}$ of Settling in 9.52-mm-1D Pipe

7.15 Pressure Drop Versus Resuspension Time for WV-205 Slurry at a $13 \mathrm{~mL} / \mathrm{s}$ Flow Rate After $20 \mathrm{~h}$ of Settling in 6.35-mIn-ID Pipe

7.16 Pressure Drop Versus Resuspension Time for WV-205 Slurry at a $45.8 \mathrm{~mL} / \mathrm{s}$ Flow Rate After $20 \mathrm{~h}$ of Settling in 6.35-mm-ID Pipe

7.17 Pressure Drop Versus Resuspension Time for WV-205 Slurry at a $57.5 \mathrm{~mL} / \mathrm{s}$ Flow Rate After $20 \mathrm{~h}$ of Settiting in 6.35-mm-ID Pipe

7.18 Pressure Drop Versus Resuspension Time for WV-205 Slurry at a $79.5 \mathrm{~mL} / \mathrm{s}$ Flow Rate After $20 \mathrm{~h}$ of Settling in 6.35-mm-10 Pipe 
8.1 Friction Factor Versus Reynolds Number for WV-205 Using the 6.35-mm-ID Pipe and the Yield Pseudoplastic Rheological

Model by Hanks

8.2 Friction Factor Versus Reynolds Number for WV-205 Using the 9.52-mm-ID Pipe and the Yield Pseudoplastic Rheological

Model by Hanks

8.3 Friction Factor Versus Reynolds Number for WV-205 Using the 12.7-mm-ID Pipe and the Yield Pseudoplastic Rheological Model by Hanks

8.4 Friction Factor Versus Reynolds Number for WV-205 Slurry with the 6.35-mm-ID Pipe

8.5 Friction Factor Versus Reynolds Number for WV-205 Slurry with the 6.35-min-ID Pipe and Fit to the Torrance Equation

8.6 Friction Factor Versus Reynolds Number for WV-205 Slurry with the 9.52-mm-ID Pipe and Fit to the Torrance Equation

8.7 Friction Factor Versus Reynolds Number for WV-205 STurry with the 12.7-mm-1D Pipe and Fit to the Torrance Equation .......

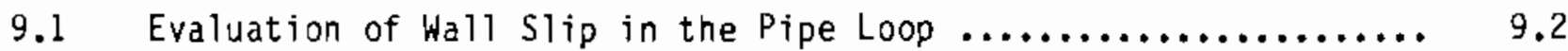

9.2 Comparison of Shear Stress Versus Shear Rate for the Haake Viscometer Using Three Different Sensors and Initial Pipe

9.3 Comparison of Shear Stress Versus Shear Rate for the Haake Viscometer Using Three Different Sensors and Later Pipe Loop Data

9.4 Comparison of Shear Stress Versus Shear Rate as Determined by the Rotational Viscometer, Capillary Viscometer, and Pipe Loop..

10.1 Logarithmic Plot of Shear Stress Versus Shear Rate Data for Water at Three Pipe Diameters Using Bowen's Method ............ 10.2

10.2 Logarithmic Plot of Shear Stress Versus Shear Rate Data for Slurry at Three Pipe Dianeters Using Bowen's Method 


\section{TABLES}

5.1 Simutated WV-205 Slurry Composition ..................... 5.4

5.2 Physical Properties of Simulated Melter Slurry at Three

Total Solids Concentrations ............................. 5.6

5.3 Settling Rates of Simulated Melter Slurry at 10 wt\%,

36 wt $\%$ and $50 \mathrm{wt} \%$ Total Solids Concentrations ................. 5.8

5.4 Rheological Constants Using the Rotational Viscometer ......... 5.20

5.5 Comparison of Linear and Nonlinear Curve Fitting Routines

Using the MVI Sensor and $36 \mathrm{wt} \%$ Total Solids Slurry at $22^{\circ} \mathrm{C} \ldots \ldots .22$

5.6 Yield Stress for Slurry Allowed to Settle for $20 \mathrm{~h}$ and

$44 \mathrm{~h}$ in the Haake viscometer ........................... 5.27

5.7 Rheological Parameters for the Slurry Supernate .............. 5..27

5.8 Summary of Rheological Parameters for WV-205 Slurry .......... 5.28

6.1 Rheological Constants for 60 wt\% Sugar Water Runs

in the Pipe Loop .................................... 6.7

6.2 Capillary Tube Rheological Parameters ...................... 6..13

6.3 Rheological Constants for STurry Runs .................... 6.17

6.4 Rheological Constants for Replicate Slurry Runs .............. 6.20

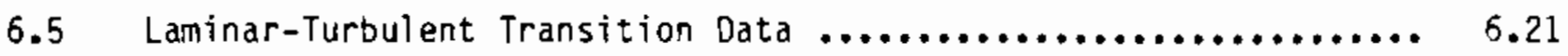

7.1 Resuspension Test Plan ................................ 7.4

7.2 Resuspension Parameters .............................. 7.18 


\subsection{INTRODUCTION AND OBJECTIVES}

The evaluation of the transport and resuspension characteristics of a simulated high-level nuclear waste slurry was conducted for the Nuclear waste Treatment Program (NWTP) at PNL. The NWTP conducts generic research aimed toward the future commercialization of high-level liquid waste (HLLW) treatment technologies. A primary objective of the NWTP is to complete development, testing, and process modeling of HLLW treatment technology. This program will help to: 1) establish the acceptability of the selected waste forms for storage and disposal, 2) verify the reliability and licensability of processes for producing the waste forms, and 3 ) demonstrate the technology by solidifying actual radioactive wastes.

The NWTP also addresses research and development needs for the HLLW treatment demonstration being conducted at West Valley, New York for the Department of Energy (DOE). Therefore, the slurry selected for evaluation in this study was a simulated West Valley waste composition. The West Valley facility was formerly operated by Nuclear Fuel Services Company (NFS) as a commercial nuclear fuel reprocessing plant.

The reference process in the United States for treating both defense and civilian HLLW is vitrification in a liquid-fed ceramic melter. A process flow diagram is shown in Figure 1.1. In the process, the HLLW is blended with glass-forming materials, and the resulting slurry is metered into a refractorylined joule-heated melting tank. There the water is driven off, and the various compounds decompose to form basic oxides that melt into a homogeneous glass. The resulting glass is poured into stainiess steel canisters for later burial in a federal repository.

The melter feed pump, as well as other pumps, must be able to transport the slurry at velocities high enough to prevent solids from depositing in the equipment. The equipment must be sized to resuspend any solids that might settle due to unplanned pump outages. The non-Newtonian behavior of the slurry complicates the evaluation of the transport and resuspension characteristics of the slurry. Experimental data and an adequate theoretical model must be available to properly size the pump in terms of flow rate and discharge pressure. 


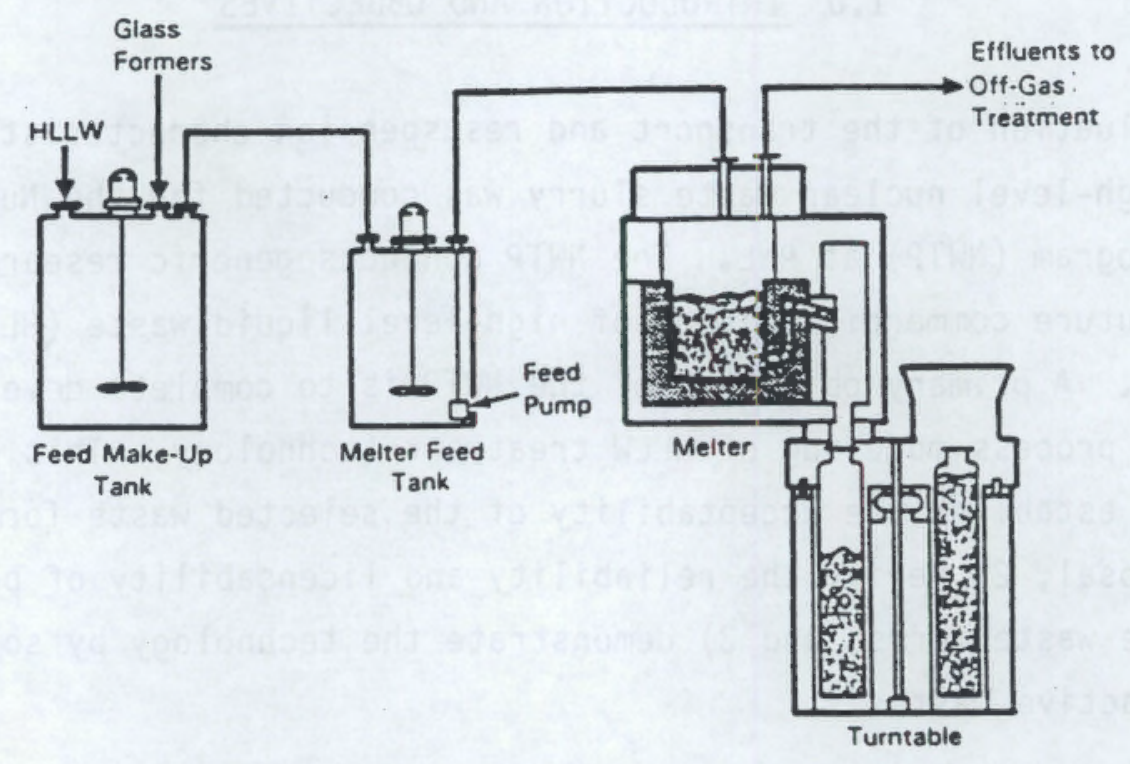

FIGURE 1.1. Vitrification Process

The specific objectives for the evaluation of the transport and resuspension characteristics of a West Valley simulated melter feed slurry were to: 1) determine the pressure drop and velocity required to resuspend settled solids in a pilot-scale pipe loop consisting of three different pipe diameters, 2) evaluate the rheological behavior of the slurry using a Haake concentric cylinder rotational viscometer, capillary tube viscometer, and data from the pipe loop experiments, 3) identify the pressure drop and velocity relationship for transporting fully suspended slurry through the pipe loop, and 4) compare the experimental results with theoretical and empirical models identified in the literature.

-Trademark of Haake Buchler Instruments, Inc. 


\subsection{CONCLUSIONS AND RECOMMENDATIONS}

The pilot-scale pipe loop experiments provided a quantitative description of the transport and resuspension characteristics of a simulated West Valley melter feed slurry. Specific conclusions and recommendations are provided below.

The evaluations used a simulated west valley melter feed slurry identified as WV-205. The physical and rheological properties of the simulated slurry were measured for this evaluation. The rheological properties were measured using both a Haake viscometer and capillary tube viscometer. In addition, the rheological properties were determined from the pipe loop experiments. The slurry evaluated in this study is a non-Newtonian fluid and can be classifjed as a yield pseudoplastic or, in some cases, a Bingham plastic fluid. All three viscosity measuring methods were in agreement at the low shear rates.

Wall slip was not found to be present in the Haake viscometer (MvI sensor), the capillary tube viscometer, or the pipe loop. The shear rate determined using the Haake viscometer must be corrected to account for non-Newtonian behavior of the slurry. The shear rates measured in the capillary tube viscometer and the pipe loop were corrected to account for non-Newtonian fluid behavior using the method of Mooney and Rabinowistch (Mooney 1931).

Resuspension of the settled slurry occurred at flowrates of 10 to $30 \mathrm{~mL} / \mathrm{s}$ in the laminar flow regime. This was an unexpected result. Typically, resuspension would not occur until turbulent flow was reached. However, further evaluation indicated that the lift and drag forces on the particles easily

overcame the gravity forces to permit resuspension in the laminar region. Resuspension at these flowrates is predicted based on literature models (Kao and wood 1974). The solids settled for $44 \mathrm{~h}$ required more time for resuspension than those that settled for $22 \mathrm{~h}$ because of greater compaction. Recommendations for further work are listed below:

- Modify the yield pseudoplastic model to correct the empirical parameter $\mathrm{B}$ to better fit the experimental data in the turbulent region. 
- Operate the pipe loop with a Newtonian fluid with a viscosity of $15 \mathrm{cP}$ to determine the pressure drop in both the laminar and turbulent regions.

- Develop a model for transient pressure drop during flow startup and resuspension and compare this model with the experimental results.

- Develop a model for predicting the yield stress of the slurry from fundamental data on the particle-particle interactions due to Van der Wals, electrostatic, and steric forces.

- Operate the pipe test loop, capillary viscometer, and Haake viscometer using a heterogeneous slurry such as coal slurry or melter slurry with larger particle sizes. Compare the three results and verify theoretical models.

- Operate the pipe loop continuously at low flow rates for long periods of time (30 days) to determine the onset and rate of sedimentation in the pipes.

Completion of this recommended work will enable a more accurate prediction of the slurry transport and resuspension characteristics in a full-scale feed system. 


\subsection{TRANSPORT OF NON-NEWTONIAN FLUIDS}

Concentrated fine particle suspensions usually behave as non-Newtonian fluids. The rheological character of a fluid is determined from a plot of the shear stress versus the rate of shear. Plots showing a linear relationship through the origin are characterized as Newtonian fluids. All other fluids are classified as non-Newtonian. There are numerous non-Newtonian fluid models for a nonlinear relationship between the shear stress and the rate of shear. The yield pseudoplastic model (sometimes referred to as the generalized Bingham plastic, yield power law, or Herschel-Buckley model) is:

$$
\tau=\tau_{y}+K\left(\frac{d u}{d r}\right)^{n}
$$

$$
\text { where } \begin{aligned}
\tau & =\text { shear stress } \\
\tau_{y} & =\text { yield stress } \\
\mathrm{K} & =\text { consistency index } \\
\mathrm{n} & =\text { flow behavior index } \\
\mathrm{du} / \mathrm{dr} & =\text { shear rate. }
\end{aligned}
$$

When the flow behavior index is one, the model reduces to the Bingham plastic model in which the consistency index is usually referred to as the coefficient of rigidity. When the yield stress is zero, the yield pseudoplastic model reduces to the pseudoplastic or power law model. When the yield stress is zero and the flow behavior index is one, the yield pseudoplastic model reduces to the Newtonian model. The West valiey slurry can be modeled as a yield pseudoplastic fluid (Peterson, McCarthy, and Muhlstein 1986), though the simpler pseudoplastic or Bingham plastic models might prove to be adequate.

If a fluid has a yield stress, another important parameter is the Hedstrom number. The Hedstrom number for a Bingham plastic model is defined as:

$$
H e=\frac{\tau_{y} D^{2} \rho}{k^{2}}
$$


where $\tau_{y}=y j e l d$ stress

$D=$ pipe diameter

$\rho=$ density

$K=$ consistency index

Hanks (1978) modified the Hedstrom number in terms of his yield power law model as follows:

$$
\mathrm{He}=\frac{D^{2} \rho}{\tau y}\left(\tau_{y} / K\right)^{2 / n}
$$

where $n=$ flow behavior index.

Hanks (1978) developed a mathematical model for yield power law fluids. Therefore, this model can be used for yield pseudoplastic fluids. This model is very complex mathematically, containing several implicit equations and one equation requiring numerical integration. The model will predict the transition velocity, Reynolds number, and the Fanning friction factor for laminar and turbulent flow. The model incorporates an empirical factor, "B", which is used to fit the turbulent flow data. For a Newtonian fluid such as water, $B=22$ (Hanks 1978). For non-Newtonian slurries such as a coal slurry, Hanks (1978) derived a relationship between $B$, the Hedstrom number, and the flow behavior index. This correlation is used in the yield pseudoplastic model to allow prediction of the Fanning friction factor versus Reynolds number plot.

The sections that follow present a theoretical background for the evaluation of slurry rheology, i.e., type of fluid (Bingham plastic, yield pseudoplastic, Newtonian), and the corresponding rheological parameters such as yield stress, flow consistency index, etc., from experiments with a pipe loop, capillary tube viscometer, or concentric cylinder rotational viscometer. In these measurements, the non-Newtonian behavior as well as wall slip should be accounted for when appropriate. Methods to adjust for these are discussed. 


\subsection{BACKGROUND FOR ROTATIONAL VISCOMETER EXPERIMENTS}

For a rotating concentric cylinder viscometer of the Searle type where the bob or center cylinder rotates while the cup or outer cylinder is stationary, the shear stress at the wall of the bob can be determined by (Skelland 1967, Schramm 1981):

$$
\tau_{b}=\frac{T}{2 \pi L R_{b}^{2}}
$$

where $T=$ measured torque on the bob

$L=$ effective length of the bob

$R_{b}=$ radius of the bob.

But unlike the capillary tube viscometer (Section 3.2), no exact mathematical equation exists for the rate of shear in terms of measurable quantities without an a priori assumption of a rheological model. For instance, for a pseudoplastic or power law fluid when the ratio of the cup to bob radius, $S$, is 1.75 or less, the relationship of shear rate to the other parameters is (Skelland 1967):

$$
(d u / d r)_{b}=\frac{4 \pi N C_{r}}{\left(1-s^{-2}\right)}
$$

where $(d u / d r)_{b}$ is the shear rate at the bob surface, $\mathbb{N}$ is the rotational speed of the bob, and $c_{r}(a)$ is a function of $S$ and the slope, $n$ ", of a logarithmic plot of torque versus rotational speed. The slope, $n^{\prime \prime}$, is defined as

$$
n^{\prime \prime}=\frac{\mathrm{d} \log T}{\mathrm{~d} \log \mathrm{N}}
$$

(a) See Skelland 1967 for a table of $C_{r}$ values. 
A modification of the above expression was given by Darby (1984) when higher order terms in the expression for $C_{r}$ are truncated:

$$
(d u / d r)_{b}=\frac{4 \pi N}{n^{\prime \prime}\left(1-s^{-2 / n^{n}}\right)}
$$

where $\quad S=R_{c} / R_{b}$, cup radius/bob radius

$$
\begin{aligned}
(d u / d r)_{b} & =\text { shear rate at the bob } \\
N & =\text { rotational speed of the bob } \\
n^{\prime \prime} & =(d \log T) /(d \log N) .
\end{aligned}
$$

For a small gap between the cylinders, i.e., $S=1.4$, and $n^{\prime \prime}$ between 0.8 and 1.5, $C_{r}$ varies from 1.077 to 0.903 . For the rotational type viscometers, the international standard is for $S$ to range between 1 and 1.10 (Schramm 1981) which means $C_{r}$ is $\sim 1$ for many fluids where the flow behavior index is between 0.5 and 1.5 .

Many of the current references (Skelland 1967, Schramm 1981) do not discuss the effect of yield stress on the calculations from rotational viscometers. If the fluid has a yield stress, $\tau_{y}$, the error in calculating the shear rate from the above equation will be maximum [around $13 \%$ in terms of $\mathrm{du} / \mathrm{dr}$, (Darby 1984)] at the point where $\tau_{b}=\tau_{y}\left(R_{c} / R_{b}\right)^{2}$ for $S=1.25$ and will decrease on either side of this point. Indeed, Darby (1984) comments that the effect of yield stress is negligible for most shear-thinning fluids.

As an example of the use of a rheological model in the determination of the relationship between rotational speed and the shear stress, consider the determination of the yield stress and consistency index in a rotational viscometer of the Searle type in which the cup is stationary and the bob rotates. Following the derivation of the Reiner-Rivlin equation in 1927 (8ird, Stewart, and Lightfoot 1960), the relationship between the stress and the velocity gradient for a Bingham plastic fluid between two concentric cylinders in which the inner cylinder rotates is:

$$
\tau_{r \theta}=\tau_{y}+k r \frac{d}{d r}\left(\frac{v_{\theta}}{r}\right)
$$


where $\tau_{\Gamma \theta}=$ momentum flux in the $r$ direction

$$
\begin{aligned}
\tau_{y} & =\text { yield stress } \\
k & =\text { consistency index or coefficient of rigidity } \\
r & =\text { radial distance } \\
v_{\theta} & =\text { angular velocity }
\end{aligned}
$$

since the momentum flux is in the $r$ direction $\left(\tau_{r \theta}=\right.$ momentum flux in the $r$ direction) and the angular velocity, $v$, decreases with $r$. The momentum flux is related to the torque exerted on the inner cylinder, $T$, the length of the bob, $L$, and the radius, $r$, by

$$
\tau_{r \theta}=\frac{T}{2 \pi r^{2} L}
$$

The stress at the cylinder wall or the bob can be found by substituting either $R_{c}$ or $R_{b}$ in the above expression. By substituting the expression for the stress into Equation (3.8) and integrating between $R_{b}$ and $r$, one obtains

$$
v(\theta)=\Omega r-\frac{r T}{4 \pi K L}\left(\frac{1}{R_{b}{ }^{2}}-\frac{1}{r^{2}}\right)+\frac{\tau_{y} r}{K} \ln \left(\frac{r}{R_{b}}\right)
$$

Where $\Omega$ is the angular speed at the bob surface. If the stress between the bob and cup falls below the yield stress, at a position $r$, there will be no motion between $r$ and $R_{c}$. For this situation, the angular speed is

$$
\Omega=\frac{\tau_{b}}{2 K}\left(1-\frac{R_{b}^{2}}{r^{2}}\right)+\frac{\tau}{K} \ln \left(\frac{r}{R_{b}}\right)
$$

If the stress remains above the yield stress throughout the region between the bob and cylinder, one obtains 


$$
\Omega=\frac{\tau_{b}}{2 K}\left(1-\frac{R_{b}^{2}}{R_{c}^{2}}\right)+\frac{\tau_{y}}{K} \ln \left(\frac{R_{c}}{R_{b}}\right)
$$

This last expression is the Reiner Rivlin equation which is the same whether the bob rotates and the cup remains stationary or the cup rotates and the bob remains stationary. For various bob sizes where the cup stress does not fall below the yield stress, a plot of stress versus rotational speed should be linear from which the consistency, $K$, can be determined from the slope and the yield stress, $\tau_{y}$, can be determined from the intercept.

For non-Newtonian fluids other behavior can complicate the analysis of rheological data. In slurries, particles can migrate away from the wall leaving a thin layer of carrier fluid. Both of these situations may lead to a reduction in apparent viscosity at the wall, resulting in conditions analogous to what would be expected if the velocity was not zero at the wall. The phenomenon is referred to as "effective" slip at the wall (Skelland 1967, Oldroyd 1960, Jastrzebski 1967).

Correction techniques for effective wall slip are not well defined for a rotational viscometer. Skelland (1967) has presented a technique to estimate wall slip in rotational viscometers. This involves the use of three different gap distances between the bob and cup to provide three values of $S ; S_{a}=R_{2} / R_{1}$, $S_{b}=R_{3} / R_{2}$, and $S_{c}=R_{3} / R_{1}$, the ratio of cup to bob radius, where $R_{1}<R_{2}<R_{3}$. For this method, both the cup and bob radius must be varied. The viscometer is then operated to allow determination of the three rotational speeds, $N_{12}, N_{23}$, $\mathrm{N}_{13}$ required to yield the same torque, $T$, for each of the respective $S$ values, $S_{a}, S_{b}$, and $S_{c}$. The effective slip coefficient at the surface at a radius of $R_{2}$ is found from:

$$
B_{2}=2 \pi^{2} R_{2}^{2}\left(N_{12}+N_{23}-N_{13}\right) / T
$$

Determination of $B_{2}$, at various values of $T$ corresponding to different $\tau_{2}$ values, allows one to plot the slip coefficient as a function of shear stress at the surface, $R_{2}$, since the shear stress at the surface is $\tau_{2}=T /\left(2 \pi R_{2}{ }^{2} L\right)$. 
The effective slip velocities for each surface, bob and cup, are calculated by muitiplying the slip coefficient by the shear stress at the appropriate surface, i.e., $u_{b s}=$ slip velocity at bob surface $=\beta_{b} \tau_{b}$. The true shear rate at the bob surface is then found by mutiplying the difference between the measured bob velocity, $u_{b}$, less the slip bob velocity, $u_{b s}$, and the slip cup velocity, $u_{c s}$, divided by the measured bob velocity, $u_{b}$, all times the shear rate, $(d u / d r)_{b}$, as determined from Equation 3.7 , i.e.,

$$
(d u / d r)_{b}(\text { adjusted })=(d u / d r)_{b}(c a l c u l a t e d)\left[\frac{\left(u_{b}-u_{b s}-u_{c s}\right)}{u_{b}}\right]
$$

In a viscometer of the Searle type (such as the Haake Rotovisco 100) the outer cylinder is stationary and serves as a temperature regulating chamber and the inner cylinder rotates. In such a device it is not possible to change the outer radius. For this device the effective slip velocity can be determined by a method recommended by $\operatorname{Van}$ Wezer (1963). For three or more gap sizes the rotational speed is determined for a given cylinder stress. The speed is then plotted as a function of $S=R_{c} / R_{b}$. The curve is extrapolated to $S=1$ at which point the rotational speed equals the sum of the slip velocity/radius at the cylinder and bob surface. The slip velocity is about one-half of the rotational speed at this point.

In a study of the rheology of coal slurries, Tsai and Knell (1986) failed to find any effect of gap width upon the apparent viscosity for Iaminar flow and for gap widths between 0.15 and $0.58 \mathrm{~cm}$ ( $S$ values between 1.14 and 1.38 ). Capillary tube experiments over the same shear rates of 20 to $800 \mathrm{sec}^{-1}$ showed wall slip. In another rotational viscometer investigation with gap widths of 0.118 and $0.035 \mathrm{~cm}$ (S of 1.07 and 1.02 ) and using a $30 \mathrm{wt} \%$ slurry of carbon black, Mannheimer (1985) found that wall slip was important, increases at both high and low shear rates, and could account for the hysteresis in the rheology plot that is considered characteristic of a thixotropic fiuid. Mannheimer's expression for determining the mean wall slip from two gap sizes, $\mathrm{H}_{1}$ and $\mathrm{H}_{2}$, for two bob sizes, $R_{1}$ and $R_{2}$, is: 


$$
u_{s m}=\frac{2 \pi\left[\left(R_{1} N_{1} / H_{1}\right)-\left(R_{2} N_{2} / H_{2}\right)\right]}{H_{1}-H_{2}} H_{1} H_{2}
$$

where $H_{1}>H_{2}$ and $u_{s m}$ corresponds to the mean stress, $\tau_{n}$, and mean shear rate, $(\mathrm{du} / \mathrm{dr})_{m}$, defined as:

$$
\begin{aligned}
\tau_{n} & =T /\left(\pi R_{n}{ }^{2} L\right) \\
(d u / d r)_{n} & =2 \pi R_{n} / H \\
R_{n} & =\left(R_{c}+R_{b}\right) / 2
\end{aligned}
$$

It appears, then, that wall slip may be important for small values of $S$, below 1.10 based upon the references above, and may account for some hysteres is effects. In conclusion, wall slip effects should be checked for in the rotational viscometer if the gap size, $S$, based upon the ratio of cup radius to bob radius, is less than 1.1 or the fluid has a very large or small flow behavior index. In addition, the shear rate should be corrected for non-Newtonian fluid behavior.

\subsection{BACKGROUND FOR CAPILLARY AND PIPE LOOP EXPERIMENTS}

The plot of shear stress versus the rate of shear in laminar flow is known as a rheogram. Capillary-tube or rotary-concentric-cylinder viscometers such as the Haake are generally used to determine the rheogram from which the parameters for the rheological models are derived. This process is straightforward and well-defined for Newtonian fluids, but is complicated for non-Newtonian fluids. For a capillary tube, the shear stress at the wall can be determined by the pressure drop $\Delta P$, over a tube length $L$ with a tube diameter $D$ using:

$$
\tau_{W}=\frac{D \Delta P}{4 L}
$$


Mooney (1931) and Rabinowitsch (1929) independently developed an equation for the rate of shear at the wall, independent of any assumption as to the rheological behavior of the fluid or slurry:

$$
\left(\frac{d u}{d r}\right)_{W}=\frac{8 V}{D}\left[\frac{3}{4}+\frac{1}{4} \frac{d \ln (8 v / D)}{d \ln (D \Delta P / 4 L)}\right]
$$

where $1 / n^{\prime}=\frac{d \ln (8 V / D)}{d \ln (D \Delta P / 4 L)}$

The assumptions for this equation are: 1) the flow is laminar at steady state, 2) the fluid is time independent, and 3) the velocity is zero at the wall which is generally referred to as "no wall slip." For a Newtonian fluid in laminar flow, $n^{\prime}=1$ which results in

$$
\left(\frac{d u}{d r}\right)_{w}=\frac{8 V}{D}
$$

For non-Newtonian fluids, the derivative, $n '$, in the Mooney-Rabinowitsch equation will vary and is determined graphically to derive the rate of shear at the wall. The pseudoshear rate is determined from measurements as $8 \mathrm{~V} / \mathrm{D}$. The slope of a logarithmic plot of shear stress, $D \Delta P / 4 \mathrm{~L}$, versus pseudoshear rate, $8 \mathrm{~V} / 0$, is determined at incremental values of shear rate. This slope is the parameter $n^{\prime}$ and is used to adjust the pseudoshear rate for non-Newtonian behavior in accordance with the Mooney-Rabinowitsch equation (3.17). The adjustment is required because the shear stress is not a linear function of shear rate for non-Newtonian fluids in a capillary tube or pipe loop.

The subsequent logarithmic plot of shear stress versus adjusted shear rate for a yield power law fluid can be used to determine the flow behavior index, $n$, from the slope at any given value of shear rate. The $y$-axis intercept of the extension of this derivative yields the $\log$ of the fluid consistency index, $K$, (Dodge and Metzner 1959). These values will not be constant unless the fluid can be modeled by a yield power law relationship. Indeed, for a yield pseudoplastic fluid, it can be shown that the slope, $n^{\prime}$, equals: 


$$
n^{\prime}=\frac{d \ln \tau_{w}}{d \ln (d u / d r)_{w}}=\left(1-\tau_{y} / \tau_{w} \cdot n\right.
$$

This equation indicates that the slope of the curve is zero when the shear stress equais the yield stress (at low shear rates) and approaches $n$ at large shear rates. The plot is concave upward.

Wall slip can be estimated, if it exists, by using different diameter capillary tubes. Presumably the effective wall slip velocity is a function of the shear stress and would vary inversely with pipe diameter at a constant flow (01droyd 1960). The plot of in $\tau_{w}$ versus in (du/dr) should yield different lines for each pipe diameter if wall slip exists. This applies to purely laminar flow. The rheogram is corrected to account for effective wall slip (Skelland 1967).

An effective slip coefficient, $\beta$, equal to $u_{s} / \tau_{W}$, where $u_{s}$ is the effective slip velocity at the tube wall and $\tau_{W}$ is the shear stress at the wall, $D \triangle P / 4 L$ is used to determine the wall slip. The steps required to determine the slip coefficient are as follows:

1. Plot $Q /\left(\pi \tau_{w} R^{3}\right)$ versus $\tau_{w}$ for laminar flow for a range of tubes of different radii but equal length. Since the velocity is finite at the wall for slip, the relationship between these groups is found by a derivation similar to the derivation of the Mooney-Rabinowitsch equation as:

$$
\frac{Q}{\left(\pi \tau_{w} R^{3}\right)}=\beta / R+\left(1 / \tau_{W}^{4}\right) \int\left(\tau_{r}\right)^{2} f\left(\tau_{r}\right) d \tau_{r}
$$

Note that the oniy term that is a function of pipe size is the term including slip, i.e., $B / R$. Therefore, for no "effective" slip, $\beta=0$, all of the curves will coincide. If slip is present, the curves will be vertically offset depending upon the pipe radius, $R$. 
2. If a series of curves is obtained, values of the ordinate, $Q /\left(\pi \tau R^{3}\right)$, are determined for each $R$ at a corresponding value of $\tau_{w}$.

3. For a constant vaiue of $\tau_{W}, Q /\left(\pi \tau_{W} R^{3}\right)$ is plotted as a function of $1 / R$ to allow determination of $\beta$ from the slope, i.e., slope $=\beta$.

4. A series of $B$ values is then found corresponding to different $\tau_{W}$ values and a plot of $B$ versus $\tau_{W}$ is made.

The flow rate is then adjusted to correct for slip by the equation

$$
Q\left(\begin{array}{c}
\text { without } \\
\text { slip }
\end{array}\right)=Q \text { (measured) }-B \pi t_{W} R^{2}
$$

This adjusted flow rate is used to find the average velocity. The average velocity can then be used to calculate the pseudoshear rate which is used in the Mooney-Rabinowitsch analysis to determine the adjusted shear rate and eventually to yield a plot of shear stress versus adjusted shear rate from which rheological parameters can be determined. The equations above will be used to check for wall slip in the pipe loop and capillary tube experiments.

wall slip has been found to vary from $\beta=0.0008 \mathrm{~cm}^{3} /$ dyn-s for a shear stress of $800 \mathrm{dyn} / \mathrm{cm}^{2}$ to $\beta=0.0172 \mathrm{~cm}^{3} / \mathrm{dyn}-\mathrm{s}$ for a shear stress of $2800 \mathrm{dyn} / \mathrm{cm}^{2}$ for $30 \mathrm{wt} \%$ kaolinite (0.55-ym particles) in water for $3.17-$, 4.76-, 6.35-mIn-ID pipes (Jastrzebski 1967). Kaolinite is a yield pseudoplastic fluid exhibiting a flow behavior index of around 0.20 and a yield stress that increases with concentration from $6.1 \mathrm{~Pa}$ at $30 \mathrm{wt} \%$ to $57 \mathrm{~Pa}$ at 50 wt\% slurry. At shear rates between 100 and $10,000 \mathrm{~s}^{-1}$, the difference between the apparent shear stress and the shear stress adjusted for slip is less than about $15 \%$ of the unadjusted value for the 6.35-mm-ID pipe and increased to about $30 \%$ for the 3.17-mm-ID pipe. The difference increased as the shear rate decreased for shear rates below $100 \mathrm{~s}^{-1}$. A similar effect of pipe diameter upon effective viscosity for laminar flow in pipes less than $6.35-\mathrm{mm}$ diameter carrying coal slurries is reported by Tsai and Knell (1986). These results indicate that wall slip may be significant for yield pseudoplastic slurries in pipe diameters less than $6.35 \mathrm{~mm}$. 
In conclusion, pipe flow data should be adjusted for non-Newtonian behavior. Wall slip should be checked and corrected to develop a sound rheological model. The need to account for non-Newtonian behavior and wall slip in many studies of slurries and the lack of studies that do account for these have lead certain investigators to prefer one type of viscometer over another. For instance, some investigators (Pouska and Link 1981) have commented, "...it seems that the rotational viscometer is better suited as a screening device where numerous samples must be studied or where only comparative results are needed. The rheometer (i.e., capillary tube viscometer) is better suited where more precise data are needed, such as for engineering design studies." Other investigators (Tsai and Knell 1986) indicate that good agreement is obtainable between the two viscometer types provided that appropriate corrections are made for the effect of $S$ and non-Newtonian fluid behavior. This is also a conclusion of Motyka and Randall (1983). They found that pressure-drop predictions based upon their unadjusted 8 rookfield rotational viscometer results were $30 \%$ higher than those observed for slurry flow through a $76.2-\mathrm{mm}-I D$ pipe; i.e., the Brookfield data should be adjusted for non-Newtonian behavior.

Both the rotational viscometer and the capillary tube viscometer may exhibit anomalies at low shear rates due to settling out of the slurry solids (Hanks 1980). This phenomenon may result in an abrupt drop of the shear stress as the shear rate decreases to zero. Such behavior often lead to the conclusion that the slurry is a yield pseudoplastic fluid when it may actually be a Bingham plastic fluid. 


\subsection{RESUSPENSION OF NON-NEWTONIAN SLURRIES}

The transport of solids in suspension has been studied for many years and there are numerous correlations for the critical design velocity (Carleton and cheng 1974). The definition of this critical velocity varies considerably among different authors, so one has to be careful of how it is defined. Carleton and Cheng (1974) have summarized many of the definitions which have been used:

- Sliding Bed Veiocity - This is the velocity at which the shearing forces in the liquid are just sufficient to move the particles lying on the floor of the pipe.

- Saltating velocity - At this velocity the particles are picked up by the liquid and deposited further along the pipe.

- Suspending Velocity - This is the velocity at which the particles are just picked up and remain in suspension and is the velocity which should be used for designing most pipelines. But it can be difficult to determine with precision, particularly if there is a wide distribution of particle sizes.

- Depositing Velocity - This is the point at which the particles settle-out as the velocity is lowered. The particles may settle as a static bed or as a moving bed. The depositing velocity is obviously related to the suspending velocity but the two are not necessarily equa 1.

- Velocity Corresponding to a Minimum in the Pressure Gradient Curve This velocity is usually referred to as the critical velocity although the term has also been used for other velocities. The minimum in the pressure gradient curve can, in theory, be located without observations of flow within the line and for this reason has proved to be a popular design velocity. However, though the choice of this velocity often gives a satisfactory design it is not certain that the velocity is always equal to or higher than the suspending velocity. 
- Velocity for Homogeneous Flow - In theory this is the velocity at which the particles become eveniy distributed throughout the pipe. In practice it is very difficult to define the transition between this type of flow and heterogeneous flow.

- Standard Velocity - This is defined as the velocity above which the pressure gradient of the suspension is equal to that for an equivalent fluid (i.e., a fiuid with the density of the suspension and the viscosity of the suspending fluid). This definition will apply only to moderate concentrations of non-flocculating solids for which the viscosity of the suspension is close to that of water. For other suspensions the pressure gradient will be greater than that for the equivalent fluid for all velocities.

- Laminar-Turbulent Transition Velocity - The transition velocity can be predicted from viscometric measurements on the homogeneous suspension. For fine particles it may be possible to operate the line at a velocity close to the transition velocity, but for larger particles the transition velocity will need to be muitiplied by a factor to obtain a design velocity. The yield power law model for homogeneous slurries (Hanks and Hanks 1984) allows one to determine the transition velocity from the determination of the rheological properties and the use of an appropriate rheological model.

Thomas (1961a) discussed the minimum transport velocity which he defined as the mean stream velocity required to prevent the accumulation of a layer of stationary or sliding particles on the bottom of a horizontal conduit. This definition is equivalent to the depositing velocity as defined above.

Many parameters which effect suspension transport have been studied. These parameters can be divided into two main areas:

- Particle parameters

- particle size and/or size distribution

- particle shape

- particle density

- particle concentration or volume fraction 
- possible particle flocculation

- interparticle forces

- settling velocity of the particles in the fluid

- particle drag coefficient.

- Hydrodynamic parameters

- carrier fluid properties--density, viscosity, yield stress

- fluid velocity and degree of turbulence

- ratio of boundary layer thickness to particle size

- shear stress at the particle fluid interface

- ratio of particle size to pipe diameter.

Many of the parameters are interrelated and there are numerous possibilities of grouping the parameters into dimensionless numbers. Early work by Shields (1936) in open channel flow grouped parameters into two dimensionless numbers :

$$
y=\frac{\tau_{w}}{g\left(\rho_{s}-\rho_{1}\right) d} \quad x=\frac{u^{\star} d}{v}
$$

where $\tau_{W}=$ shear stress at pipe wall

$d=$ particle diameter

$\rho_{S}=$ density of solid

$\rho_{1}=$ density of liquid

$u^{*}=$ friction or shear velocity

$=\sqrt{\left(\tau_{w} / \rho_{1}\right)}$

$\nu=$ kinematic viscosity

$\mathrm{g}=$ gravitational constant.

These parameters were plotted at the point of incipient particle motion (defined as the point at which a particle can at least temporarily escape the sediment bed and move in the liquid) to form the Shields diagram shown in Figure 4.1.

Later work by Thomas $(1961 \mathrm{~b}, 1962,1963)$ in pipe flow grouped parameters into four dimensionless numbers: 


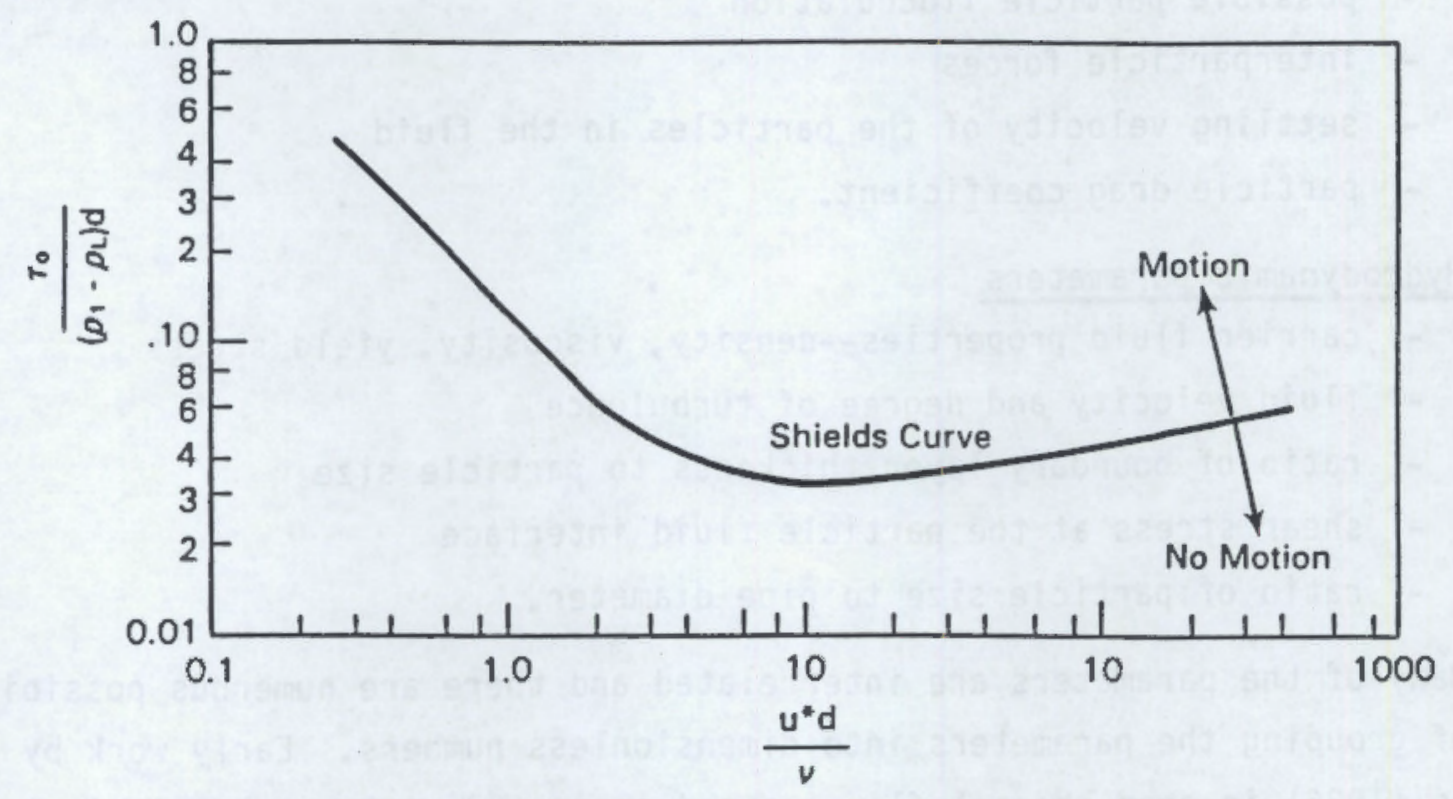

FIGURE 4.1. Shields Diagram for Particulate Transport

$$
\begin{array}{ll}
N_{1}=\frac{d u_{0}^{*}}{v} & N_{2}=\frac{d w}{v} \\
N_{3}=\frac{w}{u_{0}} & N_{4}=\frac{d}{\delta}
\end{array}
$$

where $d=$ particle diameter

$$
\begin{aligned}
u_{0}^{*}= & \text { friction velocity for limiting case at infinite dilution, } \\
& \text { determined from plotting } u^{*}=\sqrt{\left(\tau_{w} / \rho_{1}\right)} \text { as a function of } \\
& \text { particle concentration and extending to zero concentration } \\
v= & \text { kinematic viscosity } \\
w= & \text { settling velocity of particle in carrying fluid under quiescent } \\
& \text { conditions } \\
\delta= & \text { boundary layer thickness }=5 v / u_{0}^{*} .
\end{aligned}
$$

Thomas developed a generalized phase diagram for suspension transport which is shown in Figure 4.2. Thomas' dimensionless numbers are interrelated as can be seen from the phase diagram. 


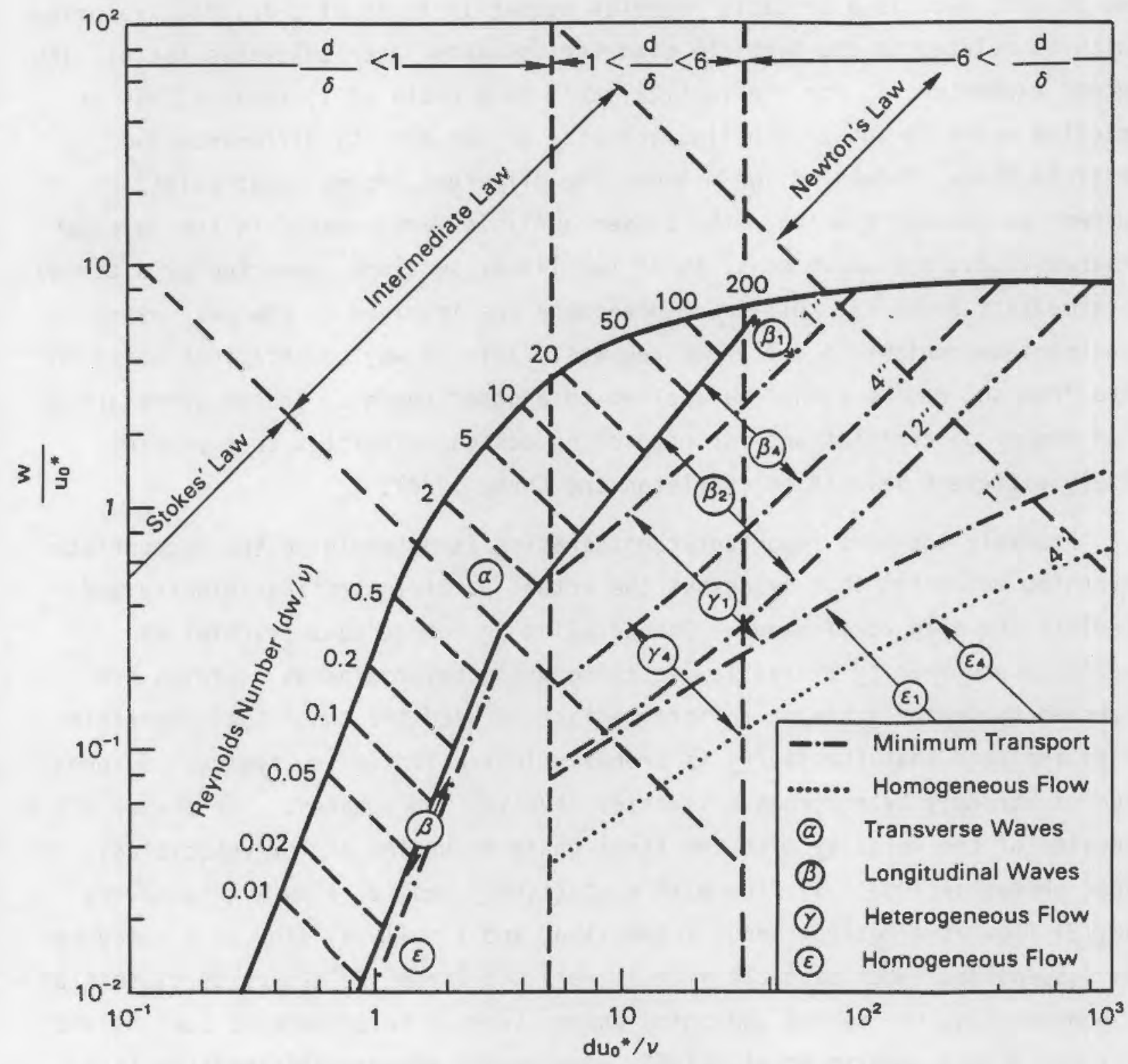

FIGURE 4.2. Generalized Phase Diagram for Transport of Suspensions. Range of $\alpha, \beta, Y$, and $(\varepsilon)$ phases apply to density ratio

Comparison and analysis of Thomas' and Shields' dimensionless numbers shows that essentially the same parameters are being used to define minimum transport or initial particle movement. The $x$ value on the horizontal axis in

(a) Adapted from Thomas 1964. Used by permission of The American Institute of Chemical Engineers. 
the Shields plot is a particle Reynolds number in terms of a friction velocity which is related to the particle diameter-boundary layer thickness ratio. The second parameter, $Y$, for the vertical axis is a ratio of 1 ) shear stress or friction velocity and 2) settling velocity or the density difference and particle size. Thomas' diagram shows the different regimes that exist in suspension transport which, with closer analysis, are evident in the Shields diagram (i.e., the curve consists of two linear sections connected by a curved intermediate section). Different phenomena are involved in the suspension transport mechanisms in different regimes. This is why correlations based on data from one regime cannot be applied to another regime. Indeed there are a wide number of correlations for predicting design velocities that predict widely different velocities (Carleton and Cheng 1977).

Probably the most important consideration is determining the appropriate governing mechanism that describes the actual particle setting velocity and predicts the drag coefficient. The settling in homogeneous slurries is typically governed by Stokes law settling while heterogeneous slurries are governed by Newton's Law or an intermediate between the two. Small particle sizes indicate that the slurry is probably in the Stokes law regime. Suspension of strongly heterogeneous slurries involves four phases. The phases are a function of the velocity with the first phase occurring at low velocities. These phases include: 1) flow with a stationary bed, 2) flow with a moving bed, 3) flow as a heterogeneous suspension, and finally 4) flow as a homogeneous suspension. When particle size is very small and the slurry is classified as homogeneous, the second and third phases seen in heterogeneous suspensions will not occur. Newitt et al. (1955) developed a diagram illustrating this (Figure 4.3). This diagram was based on only one fluid-solid system and is more qualitative than quantitative, but it does show the differences between the two slurry types. Thomas' work (1963) confirms this by stating, "For very fine particles in the micron size range, considerable data indicate that substantially homogeneous flow is achieved as soon as the minimum transport velocity is exceeded."

The vast majority of suspension transport correlations have been developed for heterogeneous slurries. The often referred to older correlations in the 


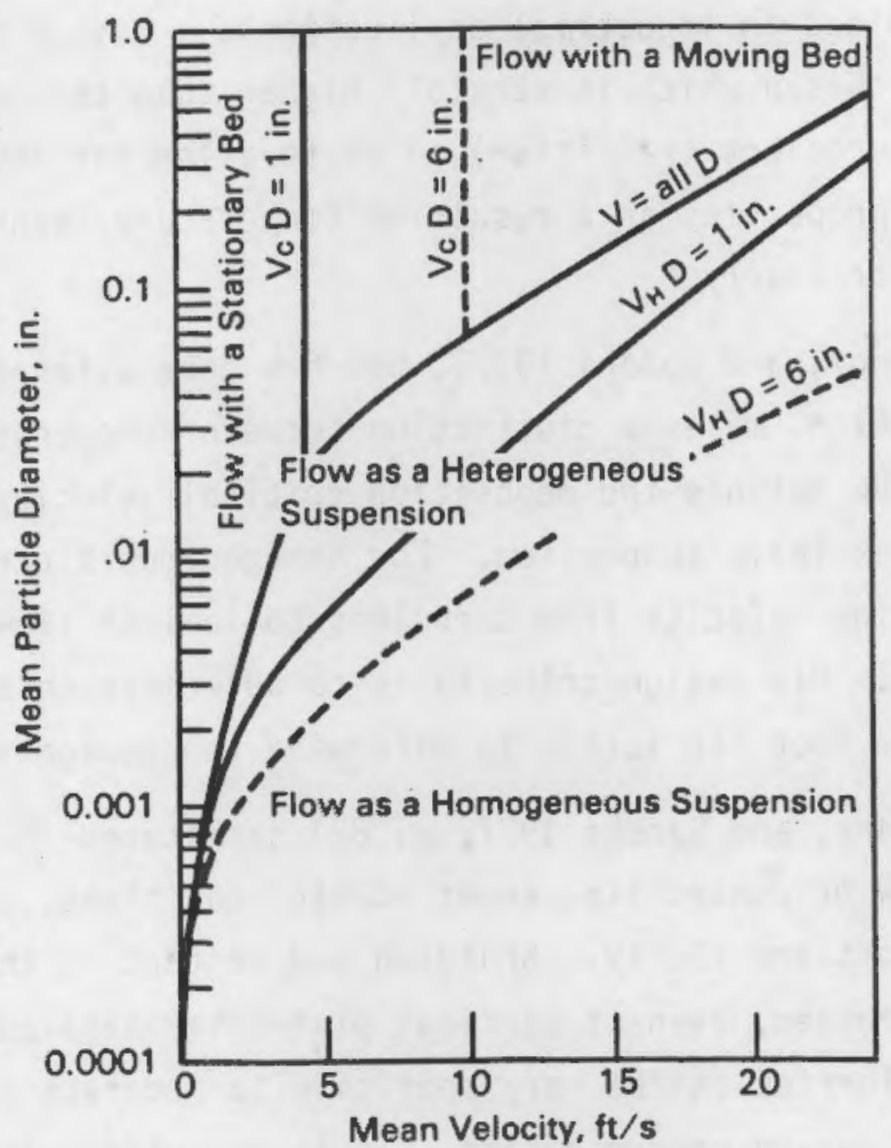

FIGURE 4.3. Mean Particle Diameter Versus Mean Velocity for Identification of Heterogeneous and Homogeneous Flow Patterns (McCarthy, Chan, and Lokken 1986)

literature such as Durand (1953) or modifications of it, Newitt et al. (1955), and Sinclair (1962) were developed for heterogeneous slurries and are not applicable to homogeneous slurries. A general model which covers both homogeneous and heterogeneous slurries remains to be developed.

Durand (1953), a pioneer in the study of sediment transport in pipes, distinguished between homogeneous (which he referred to as mud) and heterogeneous slurries. He stated, "Practically speaking, the laminar flow regimes of mud are not stable flow regimes. After a certain time, a sediment is formed in the pipe, this leading to the progressive and continuous formation of a highly concentrated solid deposit on the pipe bottom. Laminar flow regimes are 
therefore to be avoided in industrial applications... A transportation velocity should be chosen which is sensibly higher than this critical velocity (i.e., laminar to turbulent transition) so as to allow for any eventual variations in the mud's properties as a result of temperature changes and to the nature of the clay or slurry."

Wasp (Wasp, Kenny, and Gandhi 1977), who has done extensive work in slurry pipeline transportation, makes a distinction between homogeneous and heterogeneous slurries. He defines the deposition critical velocity as the velocity below which particles leave suspension. For homogeneous slurries, he equates the viscous transition velocity from turbulent to laminar flow to the critical deposition velocity. His design criteria is to determine this critical velocity and add one foot per second to determine the design velocity.

Wasp (Wasp, Kenny, and Gandhi 1977, p. 82) has stated "Homogeneous slurries tend to be slow or nonsettling under static conditions, and the settled slurry is usually soft and fluffy. Shutdown and restart of the system will probably not be a problem, even if vertical piping is involved. On the other hand, homogeneous slurries can be very sensitive to moderate concentration increases above the design concentration. It is not difficult to conceive of pumping enough high-concentration (high yield stress) slurry to exceed the system's pressure capabilities." Wasp developed a diagram shown in Figure 4.4 to illustrate the difference between homogeneous and heterogeneous slurries. Heterogeneous slurries will show a minimum in the plot of friction loss versus velocity. He noted that "Curve B shows viscous-transition critical velocity, which is characteristic of homogeneous slurries. While design of a system for operation below the transition critical velocity is acceptable for truly homogeneous slurries, no turbulent forces exist to suspend even trace amounts of heterogeneous particles."

Thomas (1961a), who developed the generalized flow regime diagram, researched the minimum transport velocity for flocculated suspensions in horizontal pipes. Flocculated suspensions, because of their low settling rates, are considered by Thomas to be representative of a homogeneous fluid. He correlated his data for concentrated suspensions in compaction (where the particles in the fluid are in continuous contact) in terms of a ratio of the 


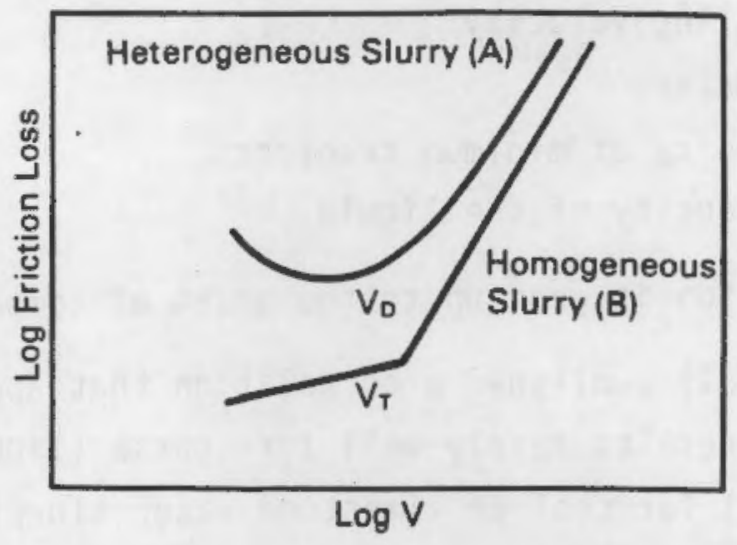

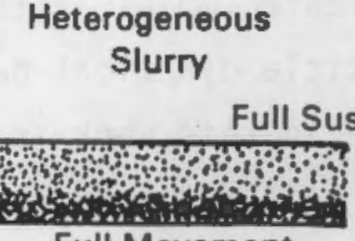

Full Movement

(A)
Homogeneous

Slurry

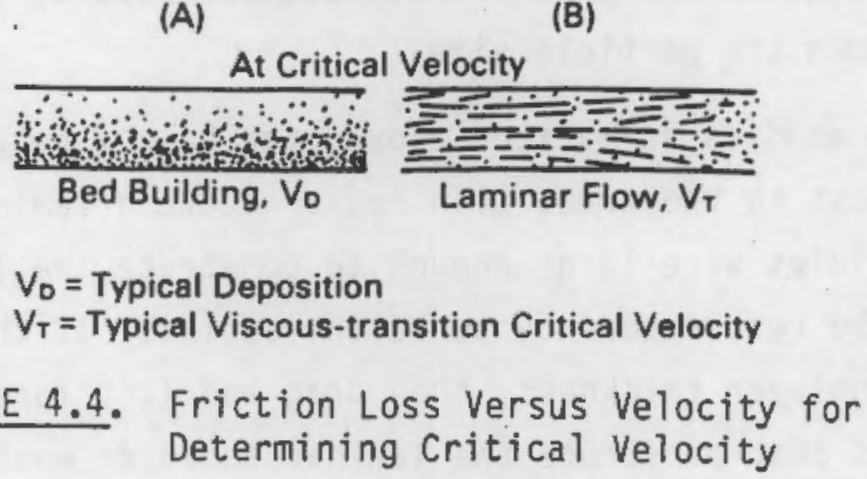

Reynolds number at the minimum transport velocity to the transition Reynolds number from laminar to turbulent flow. This ratio varied from 1.1 to 1.6. He also showed that the suspending velocity was closely related to the laminarto-turbulent transition Reynolds number when the solids concentration was sufficiently high. For more dilute slurries in hindered settling, where the particles are no longer in continuous contact, Thomas developed the following correlation:

$$
w / u_{m t}^{*}=0.0083\left(\frac{d u^{*}{ }_{m t}}{v_{1}}\right)^{2.61}
$$


where $w=$ terminal settling velocity

$d=$ particle diameter

$u^{\star}{ }_{\mathrm{mt}}=$ friction velocity at minimum transport

$v_{1}=$ kinematic viscosity of the liquid.

Thomas claims this equation is good up to the onset of compaction.

Hanks and Sloan (1981) published a correlation that appears to model minimum deposition velocity results fairly well for coarse (1400- $\mu$ m diameter) sand to fines (40-um diameter) for coal or limestone water slurries for resuspension in turbulent flows. The relatively complicated model is the first to incorporate the influence of rheological and particle dynamical behavior upon the settling velocity. The conclusion reached in this work is that a simple power law dependence of the critical deposition velocity upon the particle and pipe diameters is not adequate and that the deposition velocity for small particles depends strongly upon the particle size.

In all of the studies summarized above, the bulk flow was considered to be turbulent or at least in the transition region between laminar and turbulent flow. If the particles were large enough to penetrate the laminar boundary layer, they would be resuspended by turbulent eddies. If they were smaller than the laminar sublayer thickness, then drag and lift forces as well as turbulent fluctuations that penetrate the laminar sublayer would act to suspend them. Thomas' results (1961a) indicate for particles smaller than the laminar sublayer, the data can be correlated by a model of either solely the lift and drag forces or the turbulent fluctuation forces.

Recent research (Gadala-Maria 1979, Leighton and Acrivos 1986) indicates that viscous resuspension (resuspension under laminar flow) is possible under certain conditions. The mechanism is shear induced diffusion which arises from the random motion of particles across streamlines which occurs when a suspension is sheared. These authors equated the downward flux of particles due to gravity force to the upward flux due to shear driven diffusion. (Brownian diffusion is neglected.) This shear-induced diffusion coefficient was postulated as proportional to the local shear rate, the particle surface area and the square of the solids concentration. The model predicts that the bed will expand upon shear to some equilibrium height that varies inversely with the 
density difference and directly with the shear rate for low Shields numbers (where gravitational forces are below viscous forces). Lejghton and Acrivos conducted experiments in a parallel rotating plate device with 43- and 139-um glass beads immersed in a lubricant with viscosity of $340 \mathrm{cP}$ used to obtain laminar flow in the apparatus.

For Shields numbers below around 0.5, no noticeable bed height was noted. For Shields numbers between 15 and 60 , the bed height/particle radius ratio varied linearly with Shields number and increased from 20 to 100 . The authors propose that resuspension by shear diffusion may be important for resuspension in the viscous or laminar flow.

The characterization of slurries containing a wide variety of particle sizes is quite complex. In many cases, one rheological model is constructed based upon the measurements of the slurry flow variables. On the other hand, Hanks (1980, 1982) believes that the rheological model should be determined from the rheological evaluation using slurries consisting of various fractions of particle sizes in the suspending fluid. This general method was first proposed by Wasp, Kenny, and Gandhi (1977). Hanks feels that the fines fraction (the fraction less than about $100 \mathrm{\mu m}$ ) is generally fully suspended and contributes the most to the rheology since it has the most surface area available for interactions as compared to the coarser size particle fractions. The rheological study should start with the evaluation of the rheological parameters from a study of the fines fraction in the suspending fluid. Further tests are conducted with fractions of the suspended particles of coarser sizes until the model parameters do not change (Hanks 1982). The fines fraction then constitutes the suspending "vehicle" which acts upon the coarser fractions. The fines fraction exhibits resistance to flow by means of its effect upon the rheology while the coarser fractions affect flow resistance by means of drag forces. Hanks believes that the resistance to flow by the coarser fractions should be determined by adding up the resistances to flow from the various coarser fractions (1980).

It appears, based upon the above studies, that there is no universal model for predicting the onset of resuspension for slurries. There is a trend, however, to look more at the rheology and state of compaction of the slurry to 
allow determination of the onset of resuspension. One rheological parameter that may affect resuspension is the yield stress. One would think that settled slurries with high yield stress would require higher shear rates to result in slurry motion and resuspension. Apparently no effort has been made to correlate yield stresses with critical stresses required for resuspension. This may be a promising area for future research. In addition, the prediction of yield stresses would also be of interest. The stress required to overcome particleparticle interactions due to electrostatic, Van der Waals, and stearic forces may be determined from fundamental research into the source and magnitude of these forces for slurries composed of different particles and in different solvent environments. 


\subsection{SIMULATED MELTER FEED SLURRY PREPARATION AND CHARACTERIZATION}

The laboratory characterization of the WV-205 slurry is important to the modeling of slurry transport and to understanding resuspension phenomena. The WV-205 slurry was prepared at PNL and was subsequently taken to the University of Idaho where it was transferred to a mixing tank and agitated. Approximately $30 \mathrm{~L}$ of this slurry were withdrawn from the tank and returned to PNL to be characterized in the laboratory. This large sample was representative of the siurry used in the resuspension experiments.

The rheological and physical properties were determined for three different total solids concentrations of the WV-205 slurry. The original slurry was analyzed to have a total solids concentration of $36 \mathrm{wt} \%$. A portion of this slurry was concentrated by evaporating water to a total solids content of $50 \mathrm{wt} \%$. Another portion of the slurry was diluted with water to a total solids content of $10 \mathrm{wt} \%$.

The physical properties that were measured for each slurry concentration included the weight percent total solids, weight percent dissoived solids, weight percent suspended solids, $\mathrm{pH}$, density at $22^{\circ} \mathrm{C}$ and $50^{\circ} \mathrm{C}$, and settling rate at $24^{\circ} \mathrm{C}$ and $50^{\circ} \mathrm{C}$. Standard laboratory techniques were used to measure these properties. A particie analysis using a HIAC-ROYCO analyzer was performed, see Section 5.2.1.

Laboratory evaluations were conducted to classify the rheological behavior of the WV-205 meiter feed slurry and to determine the effects of shear rate, temperature, total solids concentration, and settling time on the apparent viscosity and yield stress of the slurry. Tests were also conducted to determine if slip at the wall in the rotary viscometer was occurring. These evaluations provided the background information needed to model slurry transport and evaluate the resuspension phenomena. The siurry composition and preparation procedures are described in Section 5.1. The physical and rheological properties of the slurry are provided in Section 5.2. 


\subsection{MELTER FEED SLURRY PREPARATION}

The slurry metered to the ceramic melter for processing consists of two fractions, HLLW and glass formers. Each of these fractions is a slurry. The waste fraction consists of the unwanted byproducts produced during nuclear fuel reprocessing. Glass formers are mixed with the raw waste to create a melter feed stream that will produce a glass product with acceptable properties. The glass formers can be added as individual glass-forming chemicals or as a glass frit. Glass-forming chemicals are usually in the form of metal hydroxides, nitrates, or oxides. Glass frit is ground glass that was produced by premelting glass-forming chemicals, cooling the resulting glass product, and milling the product to the required particle size.

The simulated melter feed slurries have been tailored to produce an acceptable glass product based on site-specific HLLW chemical and physical properties. The siurry used in the resuspension was designated WV-205 slurry. This slurry was developed for the West valley (WV) Demonstration Project at West Valley, New York. West Valley is the site of a former commercial nuclear fuel reprocessing plant. The purpose of the WV project is to vitrify the waste that was produced during reprocessing. This waste is currently stored in underground tanks.

The WV-205 slurry was selected for the resuspension experiments because this slurry type conforms with the objectives of the Nuclear Waste Treatment Program (NWTP) which is supporting this research. The NWTP focuses on generic research to permit the future commercialization of HLLW treatment technologies and to support the research and development needs of the WV project.

\subsubsection{Composition of WV-205 Siurry}

The WV melter feed slurry has been tailored to create a glass with properties that exceed all applicable regulatory requirements. The design of the slurry begins once the HLLW composition has been specified. An empirical model based on many years of HLLW glass development is used to define a first approximation of the glass composition for initial testing. Next a simulation of this HLLW is prepared. Glass formers are added to this simulated waste, the combined slurry is melted, and the properties of the resulting glass are 
analyzed. This procedure is repeated until an acceptable working glass is defined. This working glass, which becomes the reference for that waste composition, is then subjected to extensive testing on both the laboratory and large-scale melter equipment with changes being made to optimize the composition and define its tolerance to variations in waste composition. This procedure has been used to define the current reference WV melter feed slurry.

The simulated WV slurry composition used in the resuspension evaluations is show in Table 5.1. It is a working glass composition and is designated WV-205. This slurry contains glass forming chemicals and an ion exchange material. The ion exchange material, unique to the WV sTurry, is used to remove radioactive cesium from the supernate solution found in HLLW storage tanks. Nitric acid is added to the waste, glass formers, and ion exchange material to account for recycle from the off-gas treatment system and to help improve the rheological characteristics of the slurry. Sugar, a reductant, is added to control the reduction/oxidation state of the glass in the melter.

\subsubsection{Slurry Preparation Procedures}

A mixing tank with an agitator and motor sufficient to maintain solids suspension is required to prepare simulated melter feed slurries. The first step in the preparation of WV feed is to add chemicais procured as solutions to the tank. Once the agitator is started, dry chemicals (except for the $\mathrm{SiO}_{2}$ ) and the ion exchange resin are added to the tank and mixed with the liquid solutions. Approximately two-thirds of the water required is then added. The ion exchange resin is then added. An in-line homogenizer is located in a 50.8-mm recirculation line. Slurry is drawn from the tank bottorn through the homogenizer and then pumped back into the tank. The slurry in the tank is homogenized for 8 to $22 \mathrm{~h}$ to reduce the particle size of the chemicals and ion exchange resin to less than about 200 mesh and to speed the dissolution of the dry chemicals. The $\mathrm{SiO}_{2}$ required in the reference feed composition is very abrasive. This material is purchased as particles with diameters less than $0.043 \mathrm{~mm}$ (325 mesh) and added to the homogenized mixture after the homogenizer has been stopped. Finally, the remaining volume of water is added to the tank to create the reference melter feed siurry. 
TABLE 5.1. Simulated WV-205 Slurry Composition

\begin{tabular}{|c|c|}
\hline Chemical Source & Concentration, $\mathrm{g} / \mathrm{L}$ \\
\hline $\begin{array}{l}\text { Waste: } \\
\mathrm{Fe}\left(\mathrm{NO}_{3}\right)_{3} \cdot 9 \mathrm{H}_{2} \mathrm{O} \\
\mathrm{Fe}(\mathrm{OH})_{3} \\
\mathrm{FePO}_{4} \cdot 2 \mathrm{H}_{2} \mathrm{O} \\
\mathrm{NaNO}_{3} \\
\mathrm{NaOH} \\
\mathrm{Cr}\left(\mathrm{NO}_{3}\right)_{3} \cdot 9 \mathrm{H}_{2} \mathrm{O} \\
\mathrm{Cr}(\mathrm{OH})_{3} \\
\mathrm{CsNO} \\
\mathrm{ZrO}\left(\mathrm{NO}_{3}\right)_{2} \cdot 2 \mathrm{H}_{2} \mathrm{O} \\
\mathrm{Mn}\left(\mathrm{NO}_{3}\right)_{2} \cdot 4 \mathrm{H}_{2} \mathrm{O} \\
\mathrm{Ni}\left(\mathrm{NO}_{3}\right)_{2} \cdot 6 \mathrm{H}_{2} \mathrm{O} \\
\mathrm{Ni}(\mathrm{OH})_{2} \\
\mathrm{La}(\mathrm{OH})_{3} \\
\mathrm{Sm}(\mathrm{OH})_{3} \\
\mathrm{Pr}(\mathrm{OH})_{3} \\
\mathrm{Nd}(\mathrm{OH})_{3} \\
\mathrm{Y}(\mathrm{OH})_{3} \\
\mathrm{Al}(\mathrm{NO})_{3} \cdot 9 \mathrm{H}_{2} \mathrm{O} \\
\mathrm{Al}(\mathrm{OH})_{3} \\
\mathrm{KNO} \\
\mathrm{Ba}(\mathrm{OH})_{2} \\
\mathrm{Sr}(\mathrm{OH})_{2} \\
\mathrm{Ce}(\mathrm{OH})_{3} \\
\mathrm{Ca}(\mathrm{OH})_{2} \\
\mathrm{Na}{ }_{2} \mathrm{SO}_{4} \\
\mathrm{NaCl}\end{array}$ & $\begin{array}{r}11.80 \\
36.75 \\
22.98 \\
6.22 \\
0.16 \\
0.90 \\
0.79 \\
0.46 \\
23.42 \\
17.23 \\
3.11 \\
1.99 \\
0.12 \\
0.12 \\
0.12 \\
0.12 \\
0.04 \\
15.65 \\
5.14 \\
0.54 \\
0.21 \\
0.11 \\
0.63 \\
2.23 \\
2.49 \\
0.11\end{array}$ \\
\hline $\begin{array}{l}\text { Ion Exchange Material: } \\
\text { IE-96 (trademark of Union Carbide) }\end{array}$ & 39.18 \\
\hline $\begin{array}{l}\text { Glass Formers: } \\
\mathrm{Mg}\left(\mathrm{NO}_{3}\right) 2 \cdot 6 \mathrm{H}_{2} \mathrm{O} \\
\mathrm{NaCOOH} 36.98 \\
\mathrm{Na}_{2} \mathrm{~B}_{4} \mathrm{O}_{7} \cdot 10 \mathrm{H}_{2} \mathrm{O} \\
\mathrm{Li}_{2} \mathrm{CO}_{3} \\
\mathrm{TiO}_{2} \\
\mathrm{SiO}_{2} \\
\mathrm{~K}_{2} \mathrm{CO}_{3}\end{array}$ & $\begin{array}{r}25.69 \\
36.98 \\
100.13 \\
26.39 \\
3.38 \\
134.76 \\
17.63\end{array}$ \\
\hline $\begin{array}{l}\text { Miscell laneous: } \\
\mathrm{HNO}_{3} \\
\mathrm{Sugar}^{2}\end{array}$ & $\begin{array}{r}8.50 \\
24.60\end{array}$ \\
\hline
\end{tabular}


The WV-205 slurry used during the resuspension experiments was prepared during a nonradioactive pilot-scale ceramic melter (PSCM) experiment which was conducted at PNL in July 1985 and designated PSCM-21. Approximately 12,000 L of the slurry were prepared. Approximately $500 \mathrm{~L}$ of this slurry were stored in polyethylene drums for later use during these resuspension experiments.

The WV-205 slurry was stored unagitated for 6 to 9 mo. As a result of this aging, the slurry contained large clear salt crystals varying in size from 3.17 to $12.7 \mathrm{~mm}$. The size of the crystals would definitely bias the resuspension experiments. Crystals of this size will probably not exist in the actual melter feed slurry because this slurry will be continuousiy agitated and processed rapidly. Therefore, the WV-205 was sieved to remove the large unrepresentative particles. First, the entire 500-L volume of slurry was transferred from the drums to a mixing tank. The slurry was agitated to produce a homogeneous mixture. The slurry was then gravity drained through a 0.79-mm standard sieve and collected in a 50-L carboy. The solids obtained in the sieve were transferred to a bucket and a small amount of slurry added. The solids were homogenized for 20 min using a laboratory homogenizer. The homogenized mixture was then added to the carboy of slurry to maintain the original composition of the mixture. Approximately eight carboys of slurry were delivered to the University of Idaho for the resuspension experiments.

\subsection{MELTER FEED SLURRY CHARACTERIZATION}

The physical and rheological properties were measured for three solids concentrations of the WV-205 slurry. The WV-205 siurry, at an initial concentration of $36 \mathrm{wt} \%$ solids, was concentrated to $50 \mathrm{wt} \%$ solids and diluted to 10 wt\% solids. The results of the simulated melter feed slurry characterization are presented below.

\subsubsection{Physical Properties}

Standard laboratory procedures were used to measure the macro-scale physical properties of this slurry. The density, pH, and solids weight distribution results are summarized in Table 5.2. These properties were measured for the total slurry, the supernate, and the settled solids for each concentration. 
TABLE 5.2. Physical Properties of Simulated Melter Slurry at Three Total Solids Concentrations

\begin{tabular}{|c|c|c|c|c|c|c|}
\hline & \multicolumn{6}{|c|}{ Weight \% Total Solids } \\
\hline & $\frac{10}{30^{\circ} \mathrm{C}}$ & $\frac{w t \%}{50^{\circ} \mathrm{C}}$ & $\frac{36}{22^{\circ} \mathrm{C}}$ & $\frac{2 \%}{50^{\circ} \mathrm{C}}$ & $\frac{50}{320}$ & $\frac{1 \%}{50^{\circ} \mathrm{C}}$ \\
\hline $\begin{array}{l}\text { Slurry } \\
\mathrm{g} / \mathrm{cm}^{3}\end{array}$ & 1.069 & 1.072 & 1.301 & 1.291 & 1.472 & 1.470 \\
\hline Siurry, $\mathrm{pH}$ & 8.9 & NM & 8.5 & NM & 8.4 & NM \\
\hline $\begin{array}{l}\text { Dissolved Solids, } \\
\text { wt\% }\end{array}$ & 3.60 & NM & 10.73 & NM & 13.80 & NM \\
\hline $\begin{array}{l}\text { Suspended Solids, } \\
\text { wt\% }\end{array}$ & 6.27 & NM & 25.41 & NM & 36.31 & NM \\
\hline $\begin{array}{l}\text { Superna̧te Density, } \\
\mathrm{g} / \mathrm{cm}^{3}\end{array}$ & 1.025 & 1.038 & 1.110 & 1.106 & 1.228 & 1.201 \\
\hline Supernate, $\mathrm{pH}$ & 8.9 & NM & 8.5 & NM & 8.3 & NM \\
\hline $\begin{array}{l}\text { Bulk Solids Density, } \\
\mathrm{g} / \mathrm{cm}^{3}\end{array}$ & 1.188 & 1.180 & 1.358 & 1.365 & 1.468 & 1.481 \\
\hline
\end{tabular}

The bulk density was determined by allowing the slurry to settle in a graduated cylinder, removing the supernate, and determining the volume and weight of the remaining solids.

To determine the settling rate, a sample of the simulated WV-205 slurry at the three solid concentrations was shaken, placed in a $100-\mathrm{mL}$ graduate cylinder, and allowed to settle. The level of the solids-liquid interface was recorded as it descended in the cylinder. Plots of interface height versus time were prepared at temperatures of 24 and $50^{\circ} \mathrm{C}$ (Figures 5.1 and 5.2 ) for the WV-205 siurry concentration of 10,36 , and $50 \mathrm{wt} \%$ solids. The settling rates for the linear portions of the curve were determined. These are summarized in Table 5.3. The $36 \mathrm{wt} \%$ and $50 \mathrm{wt} \%$ solid concentration slurries settled very slowly probably due to the higher solids concentration and interactions. The total time allowed for settling was $20 \mathrm{~h}$. 


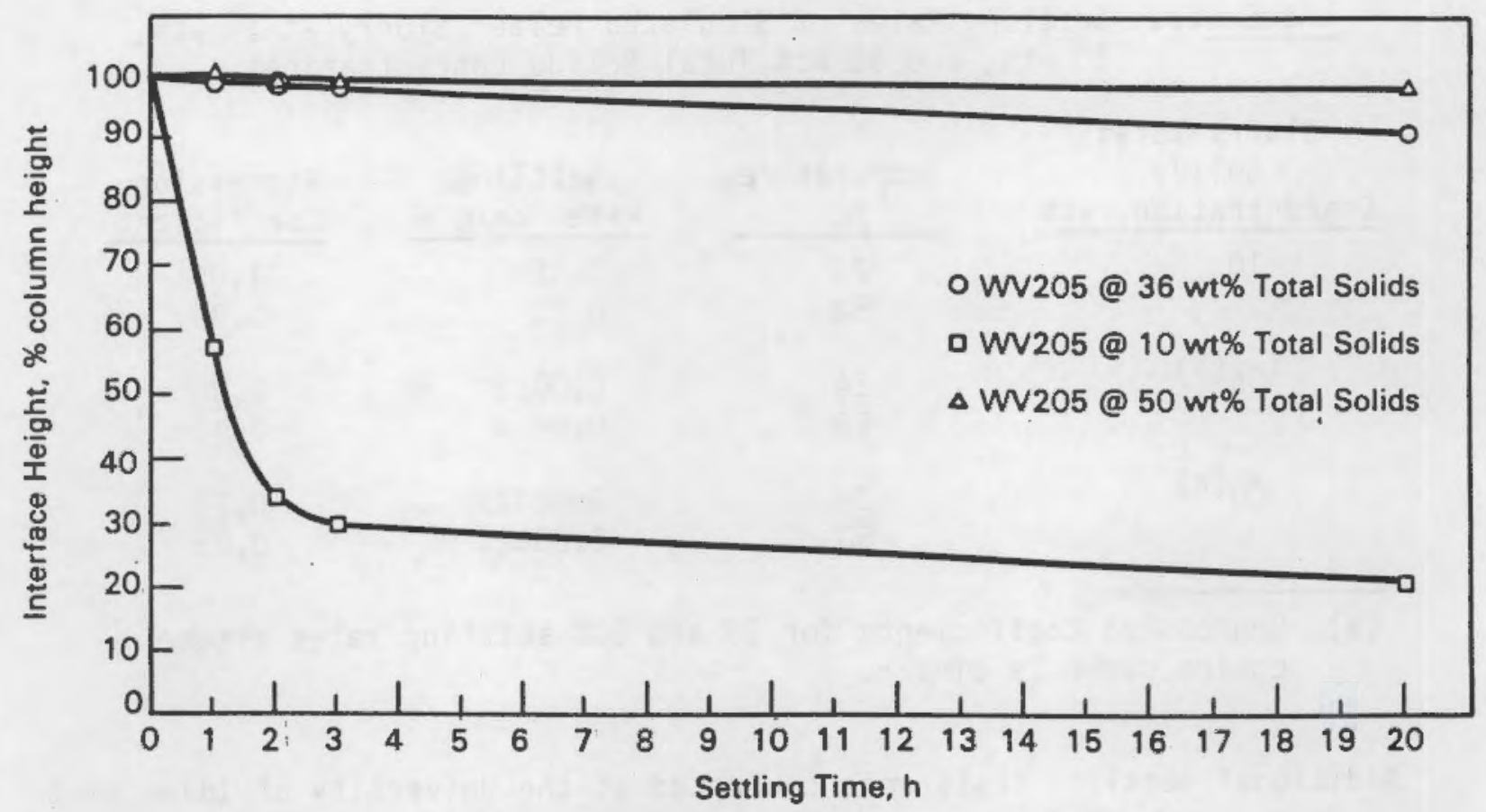

FIGURE 5.1. Settling Rate Curves for WV-205 Slurry at Three Total Solid Concentrations at $24^{\circ} \mathrm{C}$

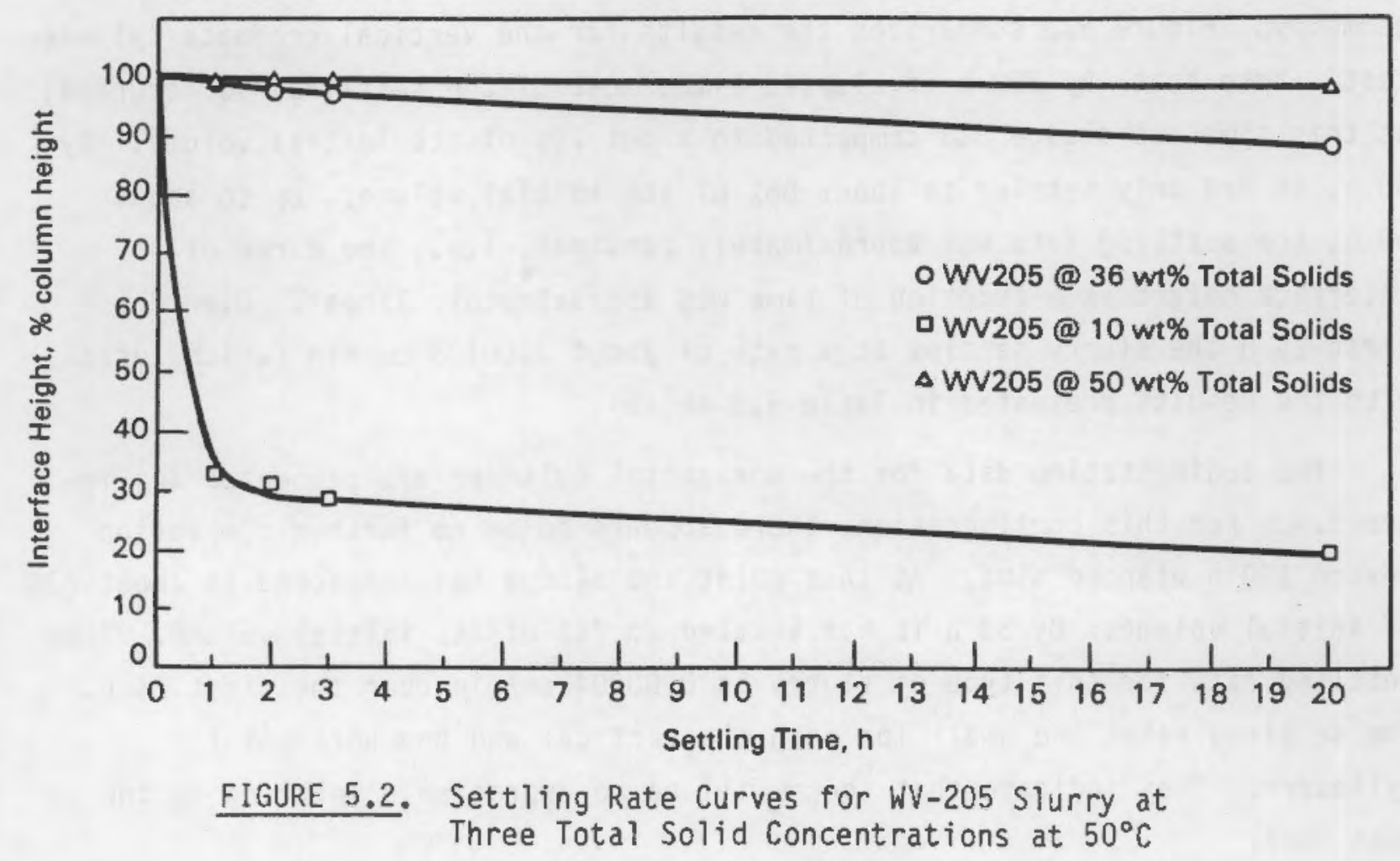


TABLE 5.3. Settling Rates of Simulated Melter Slurry at 10 wt\%, $36 \mathrm{wt} \%$, and $50 \mathrm{wt} \%$ Total Solids Concentrations

\begin{tabular}{|c|c|c|c|}
\hline $\begin{array}{c}\text { Slurry Total } \\
\text { Solids } \\
\text { Concentration, wt\% } \\
\end{array}$ & $\begin{array}{c}\text { Temperature, } \\
{ }^{\circ} \mathrm{C} \\
\end{array}$ & $\begin{array}{c}\text { Settling } \\
\text { Rate, } \mathrm{cm} / \mathrm{min} \\
\end{array}$ & $\begin{array}{l}\text { Regression } \\
\text { Coefficient } \\
\end{array}$ \\
\hline 10 & $\begin{array}{l}24 \\
50\end{array}$ & $\begin{array}{l}0.12 \\
0.21\end{array}$ & $\begin{array}{l}1.00 \\
0.99\end{array}$ \\
\hline $36^{(a)}$ & $\begin{array}{l}24 \\
50\end{array}$ & $\begin{array}{l}0.0012 \\
0.0014\end{array}$ & $\begin{array}{l}1.00 \\
0.99\end{array}$ \\
\hline $50^{(a)}$ & $\begin{array}{l}24 \\
50\end{array}$ & $\begin{array}{l}0.00021 \\
0.00026\end{array}$ & $\begin{array}{l}0.77 \\
0.85\end{array}$ \\
\hline
\end{tabular}

(a) Regression coefficients for 36 and $50 \%$ settling rates assume entire curve is linear.

Additional settling tests were conducted at the University of Idaho with the WV-205 36 wt\% slurry in a 500-mL vertical graduated cylinder and a horizontal 142-cm inside-diameter (ID) by $21-\mathrm{cm}-1$ ong plexiglass tube. Horizontal tube experiments were conducted to see if there were any wall effects due to geometry. Figure 5.3 summarizes the results for the vertical graduate cylinder test. Note that, by $200 \mathrm{~h}$ of elapsed time, most of the settling had occurred; at this time the sludge had compacted to about $70 \%$ of its initial volume. By $50 \mathrm{~h}$, it had only settled to about $86 \%$ of its initial volume. Up to about $50 \mathrm{~h}$, the settling rate was approximately constant, i.e., the curve of interface height as a function of time was approximately linear. Over the first $24 \mathrm{~h}$ the slurry settles at a rate of about $0.00126 \mathrm{~cm} / \mathrm{min}$ (which agrees with the results presented in Table 5.3 above).

The sedimentation data for the horizontal cylinder are presented in Figure 5.4. For this configuration, there appears to be no further compaction beyond $100 \mathrm{~h}$ elapsed time. At this point the sludge has compacted to about $63 \%$ of initial volume. By $50 \mathrm{~h}$ it has settled to $74 \%$ of its initial volume. The settling rate for this type of slurry is $0.00204 \mathrm{~cm} / \mathrm{min}$ over the first $24 \mathrm{~h}$. The settling rates are small for both the vertical and the horizontal cylinders. They indicate that there will be no appreciable settling in the test loop. 


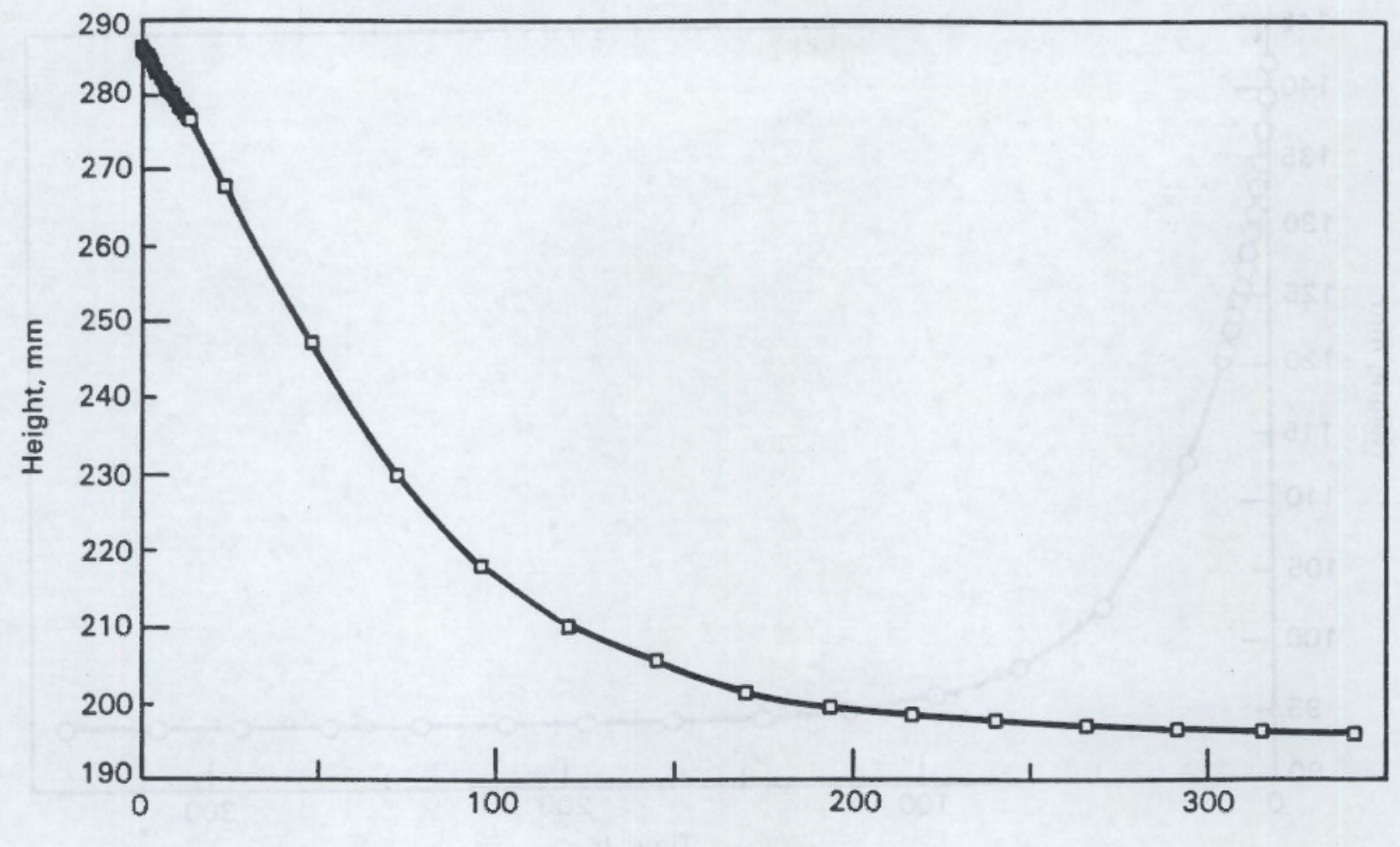

Time, $\mathrm{h}$

FIGURE 5.3. Settling Rate Determined by Measuring the Slurry Interface Height Versus Time in a Vertical Graduated Cylinder

The particle size distribution of the slurry was determined by means of a particle size analyzer with a sensor. This instrument uses an optical cell through which the diluted slurry is passed. The sensor is designed to allow single particles to pass through the cell, provided that the concentration is less than 3,500 particles/mL and the particle size is less than $60 \mu \mathrm{m}$. As a single particle passes through the cell, it is counted by the light reduction and sized in the range between 1 and $42 \mu \mathrm{m}$ by the amount of light scattered. Calibration is performed by the use of latex monodispersed spheres suspended in a media to prevent coagulation.

For determining particle size, the slurry was diluted with filtered supernatant. The supernatant was produced by filtering centrate from centrifuged slurry through Whatman \#40 paper followed by filtration through 1.2- $\mu m$ Millipore paper. Dilutions were made to yield a sample with $0.005 \mathrm{~mL}$ of 


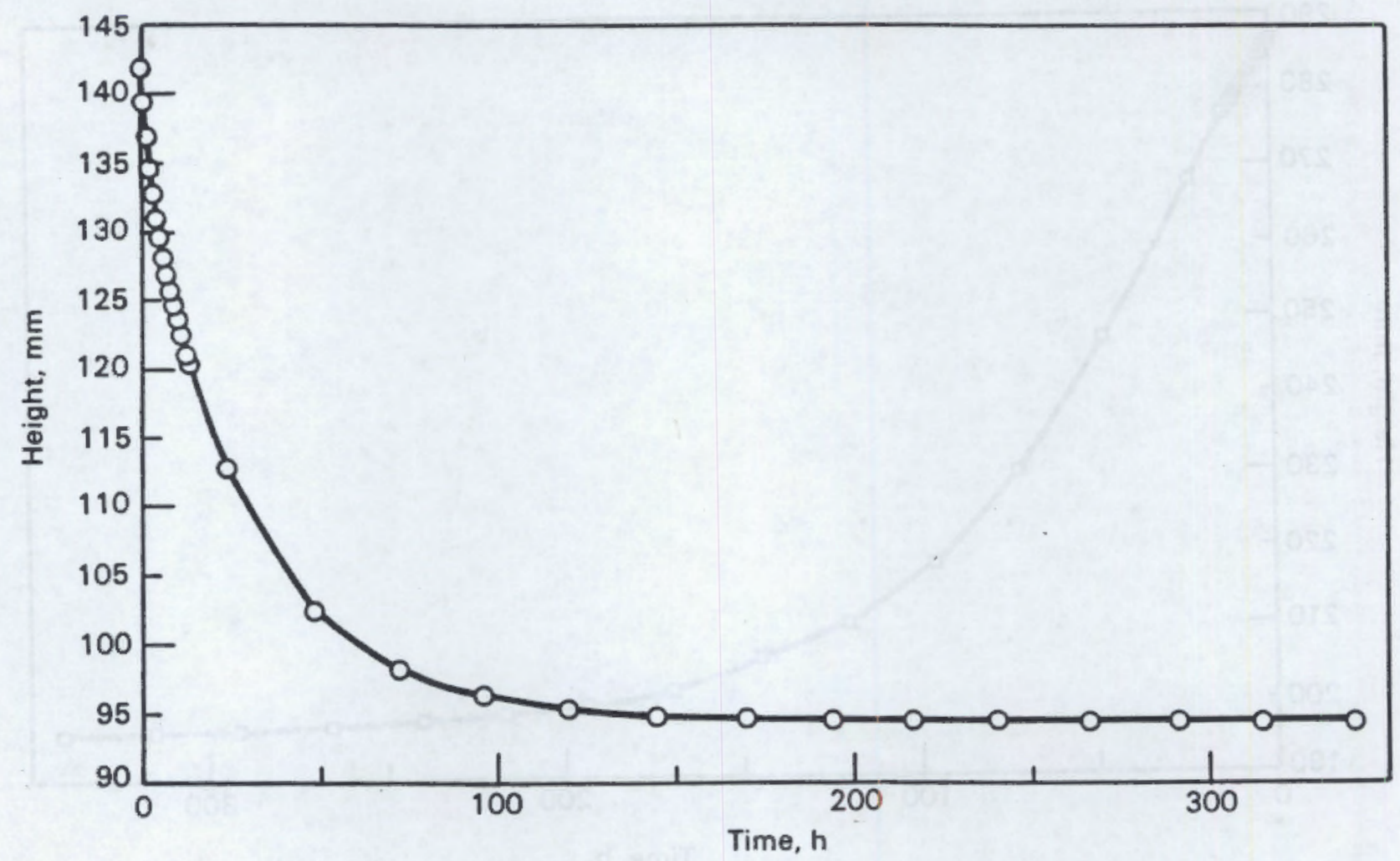

FIGURE 5.4. Settling Rate Determined by Measuring the Slurry Interface Height Versus Time in a Horizontal Cylinder

slurry/200 mL of filtered centrate. Some of the slurry was a1so wet-screened for determination of the amount of material greater than $100 \mu \mathrm{m}$.

Filtration of the uncentrifuged slurry yielded $290 \mathrm{~g}$ of dried solids $/ \mathrm{L}$ or about $29 \%$ suspended solids. The slurry was dried at $110^{\circ} \mathrm{C}$ for $24 \mathrm{~h}$. Wet screening through two screens indicated that $0.08 \%$ of the suspended solids in the slurry were greater than $600 \mu \mathrm{m}$ and $0.44 \%$ of the suspended solids were greater than $100 \mu \mathrm{m}$.

The filtered supernatant that was used to dilute the slurry yielded a concentration of 1580 particles/mL with a population mean size of $2.5 \mu \mathrm{m}$, a median size of $1.8 \mu \mathrm{m}$, and a standard deviation of 2.0. Based upon volume, the mean size is $18 \mu \mathrm{m}$ and the median is $15.8 \mu \mathrm{m}$. The filtered, distilled-water background concentration was 375 particles/mL with a population mean size of $3.3 \mu \mathrm{m}$, a median size of $2 \mu \mathrm{m}$, and a standard deviation of 2.41 . Correspondingly, the volume mean size is $22.3 \mu \mathrm{m}$ with a median of $20 \mu \mathrm{m}$. 
The sample results with filtered centrate as a diluent yielded concentrations of $1.6 \times 10^{9}$ particles/mL, a population mean of $2.2 \mu \mathrm{m}$, a median of $1.8 \mathrm{\mu m}$, and a standard deviation of 2.3. Corresponding volume statistics include a mean diameter of $11.9 \mu \mathrm{m}$, and a median of $9.2 \mu \mathrm{m}$. Determinations using distilled water as a diluent yielded a population mean size of $2.3 \mu \mathrm{m}$, a median size of $1.8 \mu \mathrm{m}$, and a standard deviation of 1.9. The corresponding volumetric mean size was $7.4 \mu \mathrm{m}$ and the median size was $5.4 \mu \mathrm{m}$. The diluent had some effect on the size distribution, with the distilled water resulting in a smaller volumetric mean particle size.

The particle mean sizes, 7 to $12 \mu \mathrm{m}$ based upon volume, compare well with results from past research. McCarthy, Chan, and Lokken (1986) obtained results of $15 \mu \mathrm{m}$ based on sieves and filtration. Peterson, McCarthy, and Muhlstein (1986) measured $9 \mu \mathrm{m}$, and Motyka and Randall (1983) measured $10 \mu \mathrm{m}$ for a similar slurry.

\subsubsection{Rheological Properties}

Laboratory rheological data were obtained with a Haake Rotovisco viscometer equipped with a measuring drive head and a sensor system. The measurement of the rheological properties with this instrument requires that the sample be placed into the gap between two coaxial cylinders (see figure 5.5). A motor drives the inner cylinder. A viscosity-related torque, caused by the resistance of the sample to shearing, acts on the inner cylinder. This torque deflects a measuring spring placed between the motor and the inner cylinder. The magnitude of the spring deflection correlates linearly with the torque. The spring deflection is transformed into an electrical signal. The torque and tachometer signals are recorded.

The rheological data was obtained using a system comprised of the M150 measuring head drive and one of four possible sensors. The sensors are designated MVI, MVII, MVIII, and NV. The sensor system is selected based on the expected fluid viscosity. Each sensor corresponds to a different gap distance and hence, different shear rates. The NV sensor, which is designed with two gaps, has the smallest gap distances, 0.35 and $0.4 \mathrm{~mm}$, and is used for fluids

Trademark of Haake Buchler Instruments, Inc. 


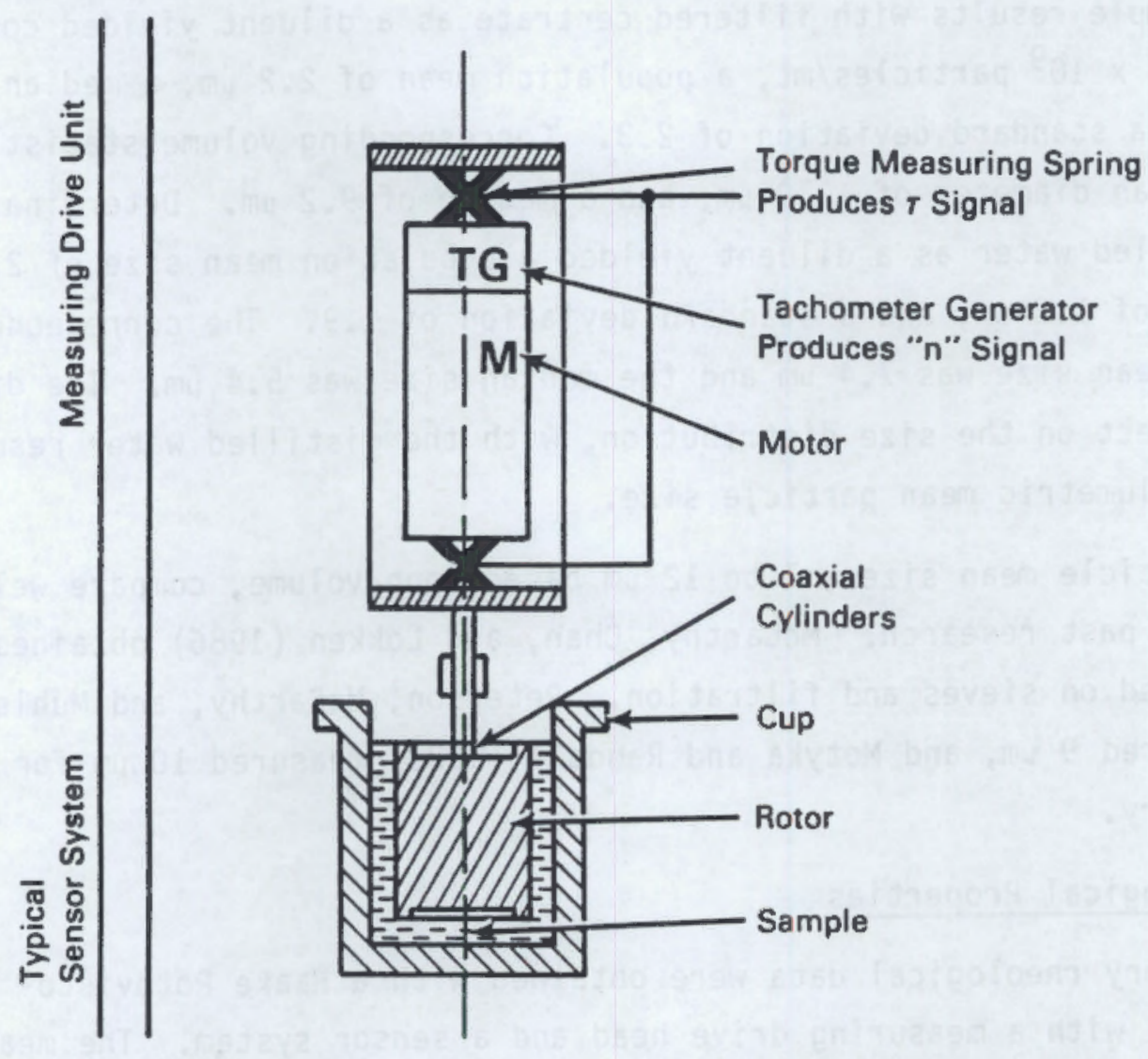

FIGURE 5.5. Schematic of a Haake Viscometer

with viscosities of approximately $1 \mathrm{cP}$. The MVI has a gap distance of $0.96 \mathrm{~mm}$, the MVII has a gap distance of $2.6 \mathrm{~mm}$, and the MVIII has a gap distance of $5.8 \mathrm{~mm}$. A smaller gap distance corresponds to more surface area and greater sensitivity. A larger gap distance can be used with a viscous slurry to obtain accurate data.

Three different gap distances (i.e., sensor systems MVI, MVII, and MVIII) were used for each concentration of slurry at the two temperatures. The NV sensor was used for characterization of the supernate. The results of the slurry tests would indicate whether or not slip at the wall of the rotor was occurring. Data from the larger gap size sensors may require correction for gap size. The MVI sensor, which has the smallest gap size of the three sensors $(0.96 \mathrm{~mm})$, has the largest surface area and produces the best results. 
The speed signal required for the determination of the shear rate does not come from the rotor directly but from the tachometer generator (TG) which sits above the motor and is mechanically linked to it. For slurries that do not exhibit yield stresses, the speeds of the $T G$, motor, and rotor, are identical so that the speed signal, $\mathrm{N}_{t g}$, also defines the rotor speed, $\mathrm{N}_{r}$. A slurry with a yield stress will "clamp" the rotor to the cup, until the torque, T, applied is greater than the yield stress, $\tau_{y}$.

While the rotor is still clamped and remains at standstill, the motor rotation will cause some spring deflection and consequently a torque signal, $T$, on the ordinate. For a slurry with a yield stress, a straight line, leaning off the vertical with an angle, $B$, is recorded initially (see Figure 5.6). When the shear stress surpasses the yield value, $\tau_{y}$, the rotor breaks loose from its "clamp", the rotor picks up speed, accelerating almost instantaneously to the value, $\mathrm{N}_{\mathrm{tg}}$. The change in the behavior of the sample becomes apparent in the rapid turn of the flow curve. The ordinate value of this turning point

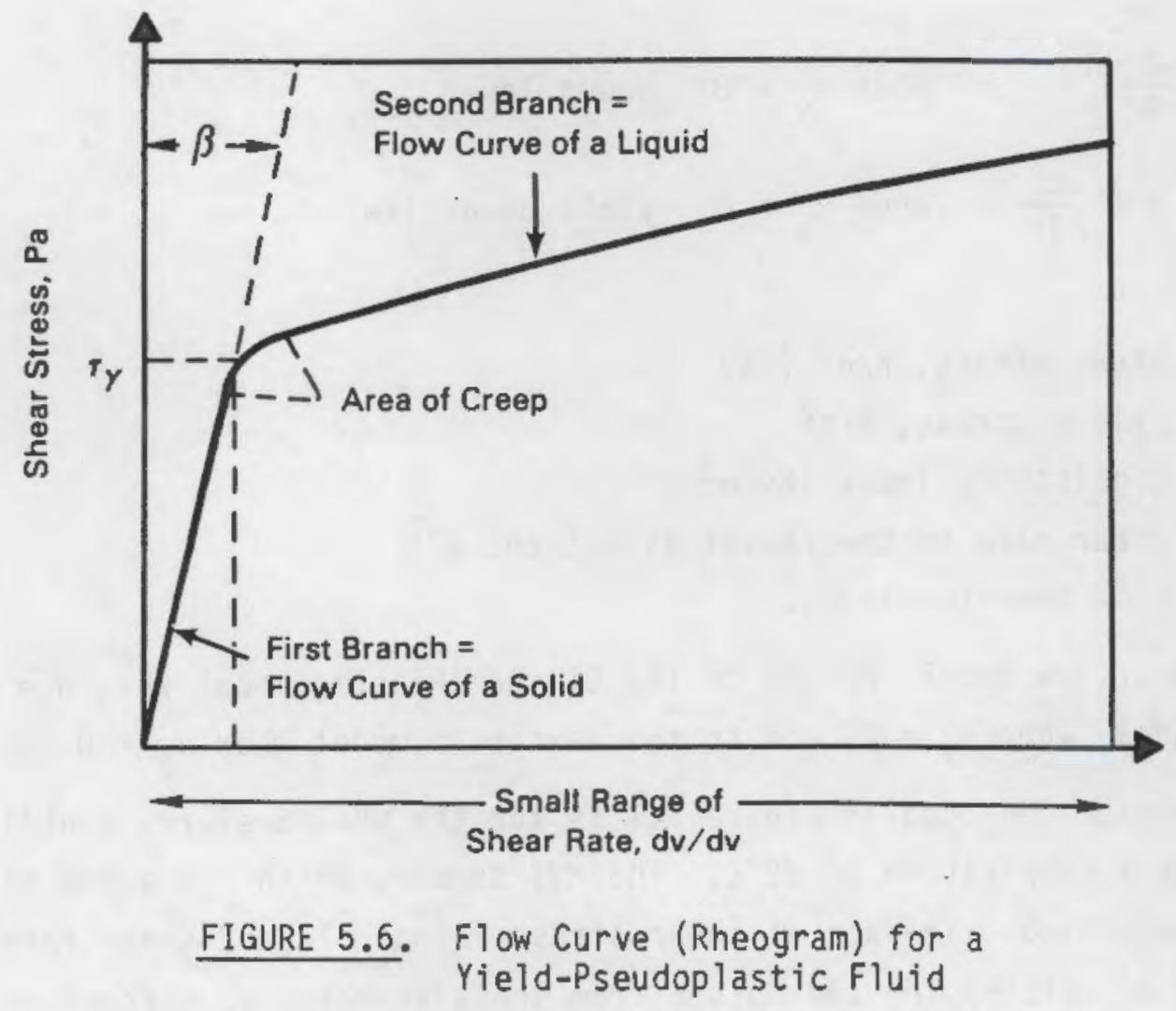


defines the yield value, $\tau_{y}$. On the flow curve two regions can be distinguished: the first region is a segment of the "flow curve of a solid," while the second region describes the flow of the material after it had been transformed into a liquid.

Depending on the test conditions selected, one may also get a more or less gradual change of the flow curve from one region to the other. The transition from a solid gel to an easy-flowing liquid may go through a phase of creep, which makes the turning point less clear. Under these conditions the yield point is determined by the crossing point of the tangents on both branches.

The WV-205 slurry exhibits basically pseudohomogeneous flow behavior as a result of the small particle size and slow settling rate. A wide variety of pseudohomogeneous flow regimes are possible. The majority of slurries can be treated as if they were power law or yield power law materials. The behavior of these materials can be defined by the following equations (Herschel and Bulkley 1926):

$$
\begin{array}{lll}
\tau=K\left(\frac{d u}{d r}\right)^{n} & \text { when } \tau_{y}=0 \text { power law } \\
\tau=\tau_{y}+K\left(\frac{d u}{d r}\right)^{n} & \text { when } \tau_{y}>0 \text { yield power law }
\end{array}
$$

$$
\text { where: } \begin{aligned}
\tau & =\text { shear stress, } N / n^{2}(\mathrm{~Pa}) \\
\tau_{y} & =\text { yield stress, } N / n^{2} \\
K & =\text { consistency index, Ns/ } n^{2} \\
(\mathrm{du} / \mathrm{dr}) & =\text { shear rate in the radial direction, } \mathrm{s}^{-1} \\
n & =\text { flow behavior index. }
\end{aligned}
$$

The yield power law model reduces to the Bingham Plastic model when $n=1$, the power law model, when $\tau_{y}=0$, and to the Newtonian model when $\tau_{y}=0$ and $n=1$.

The rheogram depicted in Figure 5.7 is for the WV-205 slurry containing 36 wt\% solids at a temperature of $22^{\circ} \mathrm{C}$. The MVI sensor, which has a gap distance of $0.96 \mathrm{~mm}$, was used. The actual shear stress in pascals and shear rate in $\mathrm{s}^{-1}$ are obtained by multiplying the output from the viscometer by correction 
factors. The correction factors account for sensor geometry and sensitivity and are specified in the operating manual for the viscometer.

The slurry sample is placed in the gap between the two cylinders. The rotor begins to turn after $2 \mathrm{~min}$. The interim period permits the sample to reach the temperature of the constant-temperature water bath surrounding the outer cylinder. The rotor speed is slowly increased to the maximum desired shear rate. This time is set for $5 \mathrm{~min}$. The rotor speed is then decreased to a shear rate of zero to evaluate the time-dependent behavior of the slurry. Figure 5.7 indicates slightly thixotropic behavior. Values from the "up" or increasing shear rate curve were used for the determination of rheological constants. Comparison of the rheograms for different slurry concentrations and

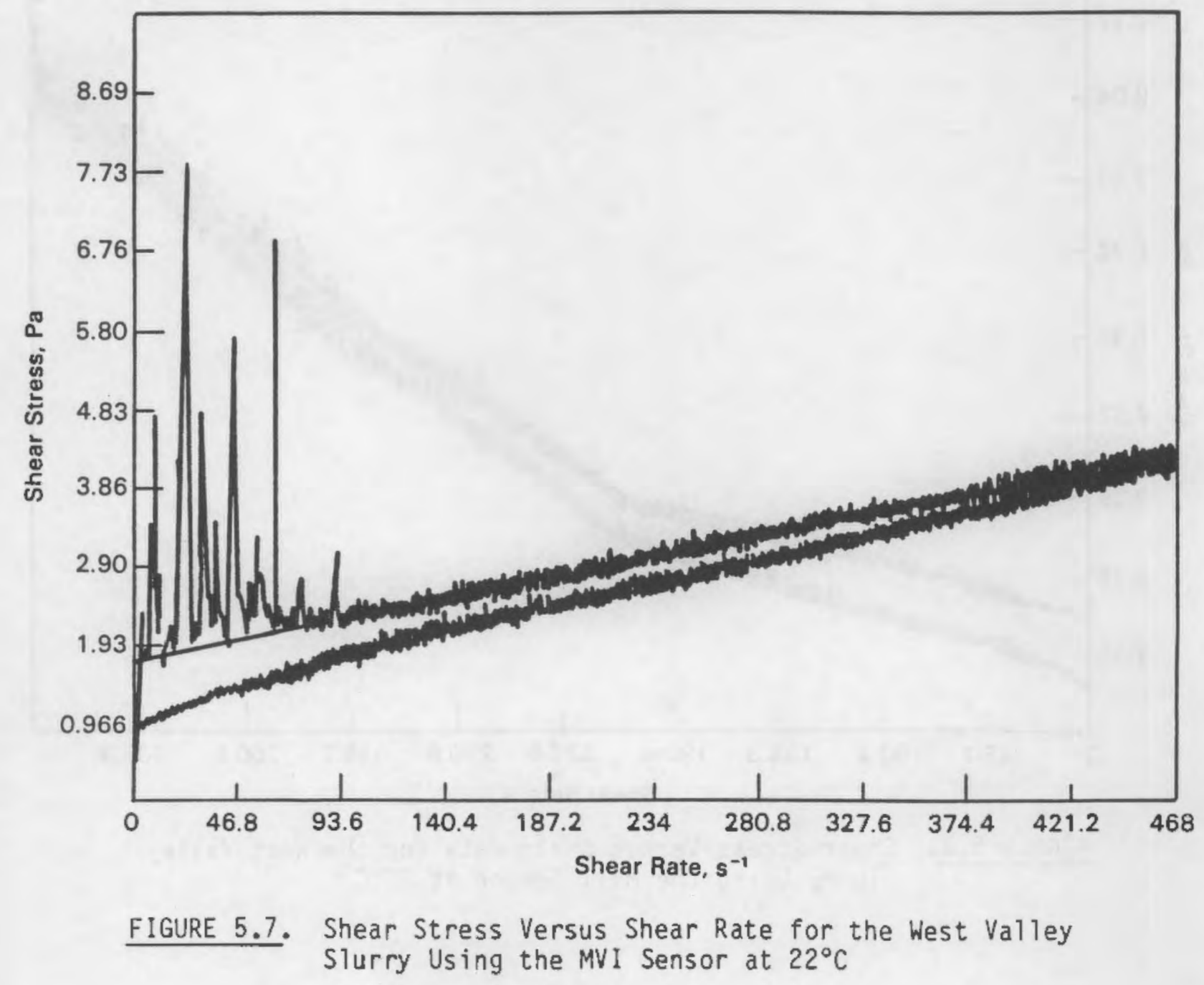


over different shear rates indicates the "up" curve is approximately $1 \mathrm{~Pa}$ above the "down" curve at the lowest applied stress. This implies a mechanical hysteresis rather than a rheological one. The slurry under a sustained, constant shear rate over a five-minute period showed no variation in shear stress.

The rheograms for the MVII and MVIII sensor differ from those of the MVI. (See Figure 5.8 for the MVII sensor.) For the larger gap size sensors, there is a break in the curve at approximately $200 \mathrm{~s}^{-1}$. This break indicates the onset of turbulent flow in the viscometer or the formation of Taylor vortices.

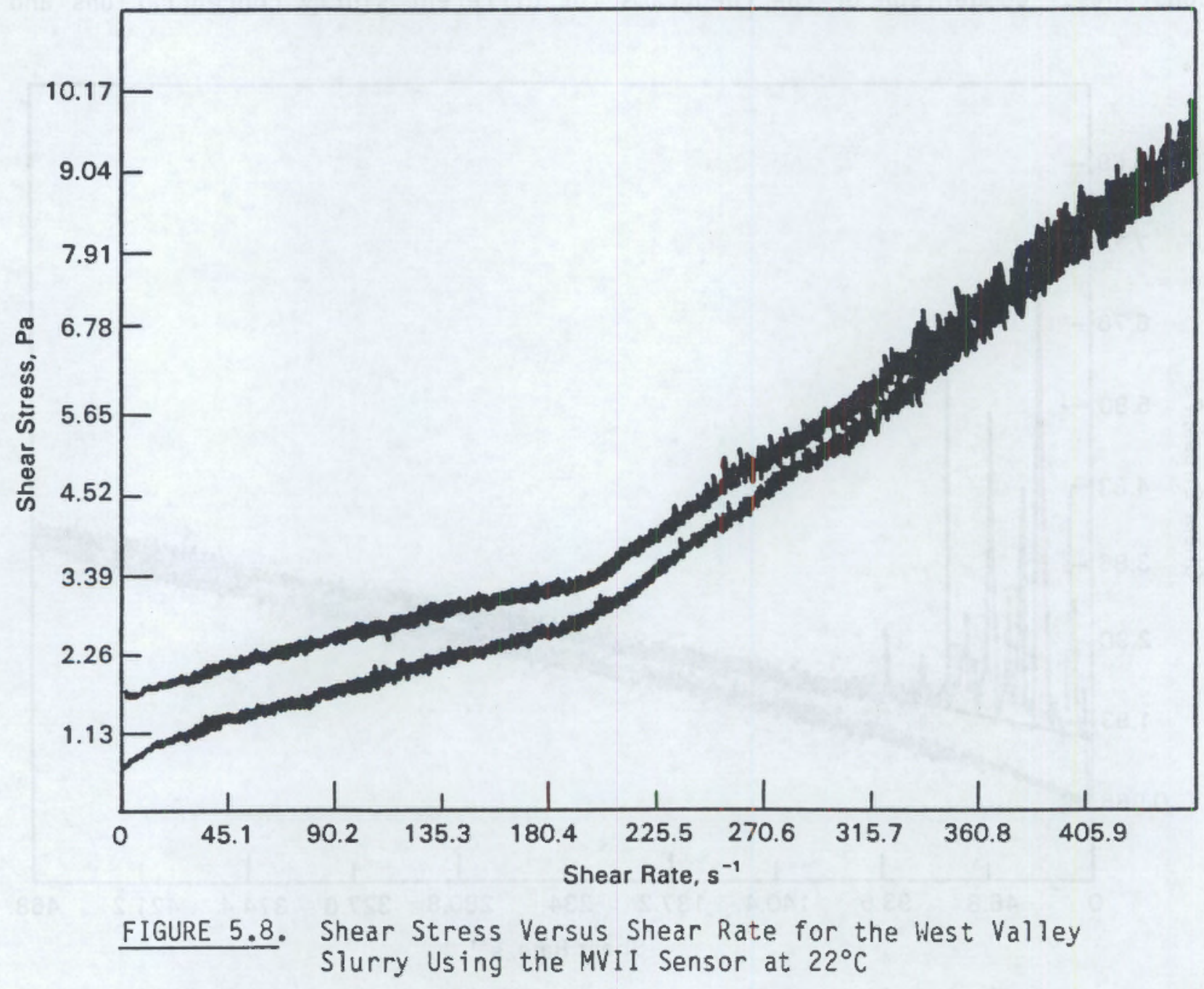


The Searle type viscometer with a rotating bob is not stable to the formation of Taylor vortices or roll cells (Schlichting 1968). The Taylor number is defined as

$$
T a=\frac{v\left(R_{c}-R_{b}\right) \rho}{v}\left(\frac{R_{c}-R_{b}}{R_{c}}\right)^{1 / 2}
$$

where $v$ is the bob angular velocity and $\nu$ is the viscosity. These cells exist in laminar flow but since energy must go into their formation, one would expect an increase in the shear stress as noted in the rheograms. Taylor vortices or roll cells occur at a Taylor number of approximately 41.3. Turbulent flow commences at a Taylor number of 400 and becomes fully developed around a Taylor number of 3800 . The commencement of turbulent flow would also result in a break in the shear stress-shear rate curve.

The break in the MVII and MVIII sensors occurred at an angular speed of around $40 \mathrm{rad} / \mathrm{s}$. Using the apparent viscosity of the slurry of $20 \mathrm{cP}$ at this rotational speed results in a Taylor number of around 40 , which indicates that the break corresponds to the onset of Taylor vortex formation. Therefore, only the rheogram data below the break point were used in the determination of the rheological parameters.

Wall slip for the rotational viscometer data was determined in the following manner. Shear stress and shear rate were calculated using the correction factors supplied by the viscometer manufacturer. Based upon this value, the angular speed was determined from:

$$
\Omega=\frac{\left(\frac{d u}{d r}\right)}{2}\left(1-\frac{R_{c}^{2}}{R_{b}^{2}}\right)
$$

where $\left(\frac{d u}{d r}\right)=$ shear rate

$$
\begin{aligned}
& R_{c}=\text { radius of the cup } \\
& R_{b}=\text { radius of the bob }
\end{aligned}
$$

A plot was made of angular speed, $\Omega$, versus cylinder stress calculated from bob stress by $\tau_{c}=\tau_{b}\left(R_{b} / R_{c}\right)^{2}$ (Figure 5.9). The plot shows a linear variation of $\Omega$ 


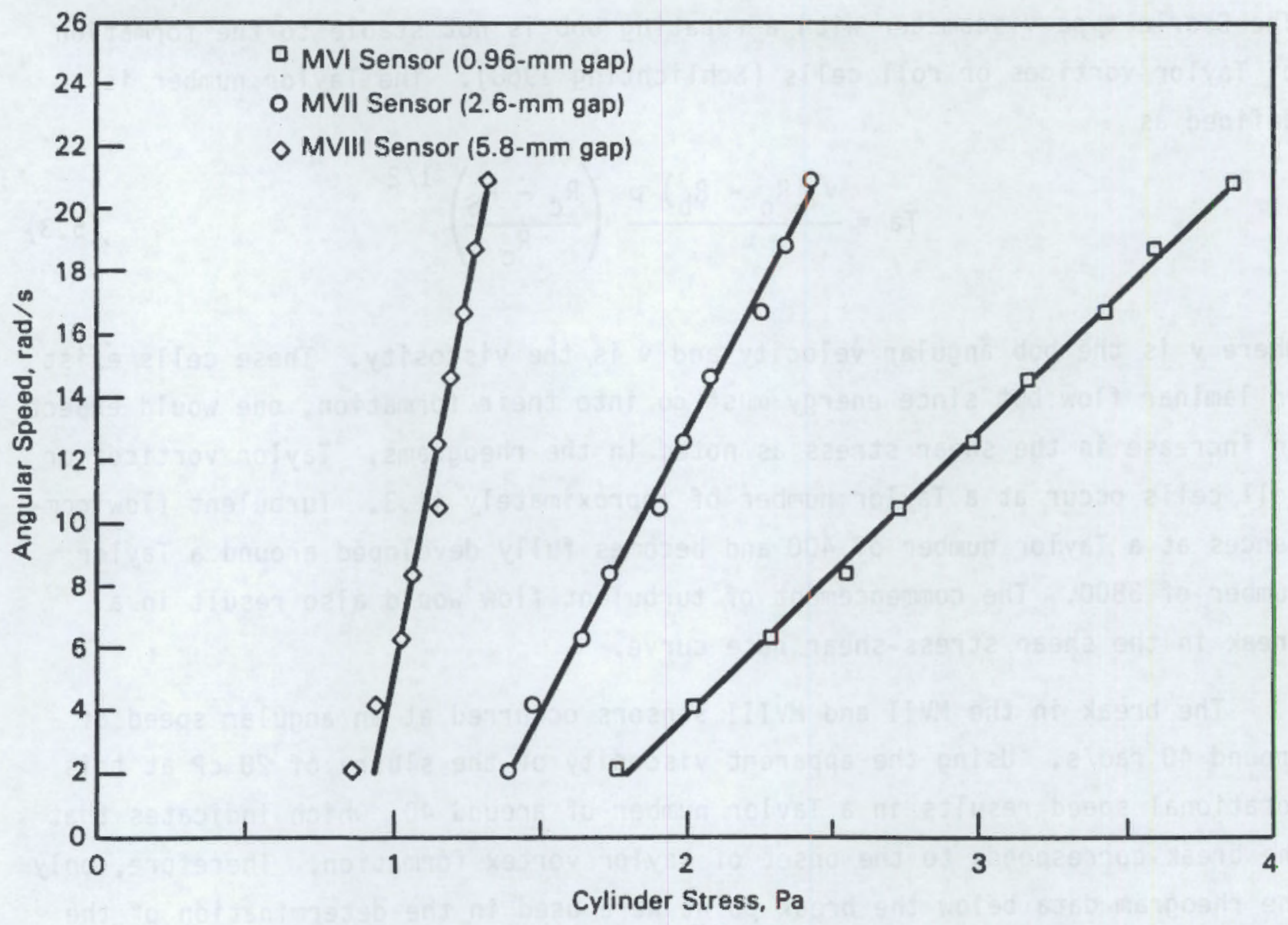

FIGURE 5.9. Angular Speed Versus Rotational Cylinder Stress for the WV-205 Slurry Using Three Different Sensors

with $\tau$ as predicted for a Bingham plastic fluid by the Reiner-Rivlin equation (Bird, Stewart and Lightfoot 1960). A linear regression was performed using the data from each sensor; from this regression, the angular speed corresponding to each of the bob sizes at the same cylinder stress was determined. This speed was then plotted as a function of $R_{c} / R_{b}$ in Figure 5.10 . The curves approach $\Omega=0$ at $R_{c} / R_{b}=1$, which indicates that there is little or no wall slip for the WV-205 slurry.

The linear variation of $\Omega$ with $\tau_{c}$ indicates that the slurry may behave as a Bingham plastic within the range of shear stresses applied. Equation (3.12) was used to correlate the data and obtain the yield stresses and consistency index for the slurry. The results are depicted in Table 5.4. These results were calculated in the manner described below. 


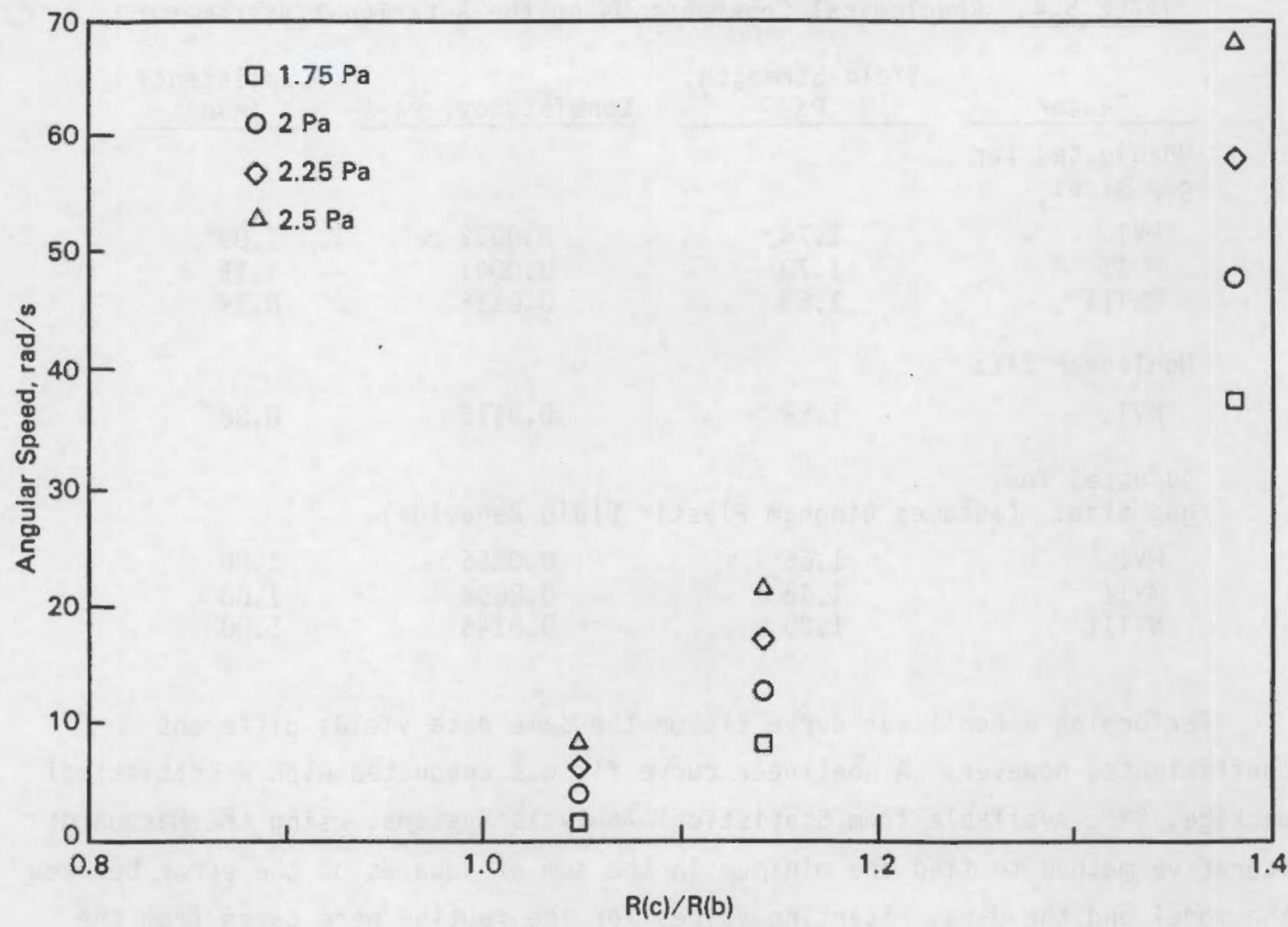

FIGURE 5.10. Angular Speed Versus the Ratio of Cup Radius to Bob Radius at Various Rotational Cylinder Stresses

The recommended method for determining yield-pseudoplastic parameters from this data involves the determination of the yield stress from the interception of the sharp rise of the curve that is characteristic of deformation of a solid and an extrapolation of the linear portion of the curve for the fluid behavior. The yield stress is then subtracted from the observed stress. A linear regression is then conducted on the logarithm of the adjusted shear stress as a function of the logarithm of the adjusted shear rate. Data manipulated in this fashion indicate that for the MVI sensor the yield stress for the 36 wt\% WV-205 slurry at $22^{\circ} \mathrm{C}$ is $1.74 \mathrm{~Pa}$, the consistency index is 0.0032 , the flow behavior index is 1.09 , and the regression coefficient $\left(r^{2}\right)$ is 0.9933 . 
TABLE 5.4. Rheological Constants Using the Rotational Viscometer

\begin{tabular}{|c|c|c|c|}
\hline Sensor & $\begin{array}{c}\text { Yield Strength, } \\
\mathrm{Pa}\end{array}$ & Consistency, $\mathrm{Pa} \cdot \mathrm{S}$ & $\begin{array}{c}\text { Consistency } \\
\text { Index }\end{array}$ \\
\hline \multicolumn{4}{|c|}{$\begin{array}{l}\text { Unadjusted for } \\
\text { gap size: }\end{array}$} \\
\hline $\begin{array}{l}\text { MVI } \\
\text { MVII } \\
\text { MVIII }\end{array}$ & $\begin{array}{l}1.74 \\
1.70 \\
1.63\end{array}$ & $\begin{array}{l}0.0032 \\
0.0041 \\
0.0116\end{array}$ & $\begin{array}{l}1.09 \\
1.15 \\
0.99\end{array}$ \\
\hline \multicolumn{4}{|c|}{ Nonlinear fit: } \\
\hline MVI & 1.59 & 0.0116 & 0.88 \\
\hline
\end{tabular}

Adjusted for

gap size: (assumes Bingham Plastic Fluid Behavior)

$\begin{array}{llll}\text { MVI } & 1.65 & 0.0056 & 1.00 \\ \text { MVII } & 1.46 & 0.0098 & 1.00 \\ \text { MVIII } & 1.20 & 0.0146 & 1.00\end{array}$

Performing a nonlinear curve fit on the same data yields different coefficients, however. A nonlinear curve fit was conducted with a statistical package, SAS, available from Statistical Analysis Systems, using the Marquardt iterative method to find the minimum in the sum of squares of the error between the model and the data. Starting values for the routine were taken from the linear regression values mentioned above. The nonlinear fit routine resulted in a yield stress of $1.59 \mathrm{~Pa}$, a consistency index of $0.0116 \mathrm{~Pa} \cdot \mathrm{s}$, a flow behavior index of 0.88 , and a regression coefficient of 0.999 . The sum of squares for the error for this model was 0.0054 as compared to 0.2338 for the linear model discussed above. The results of both models and the data appear on Figure 5.11. The data is tabulated in Table 5.5. Note that both models correlate the data fairly well although the nonlinear model has slightly better agreement. The nonlinear regression method is preferred due to the better statistical fit to the data.

The rheological behavior was determined for each of the slurry concentrations at both $22^{\circ} \mathrm{C}$ and $50^{\circ} \mathrm{C}$. The shear stress versus shear rate graphs are shown in Figures 5.12 and 5.13. The MVI sensor that had the smallest gap distance and hence, yielded the more accurate results was used to obtain these 


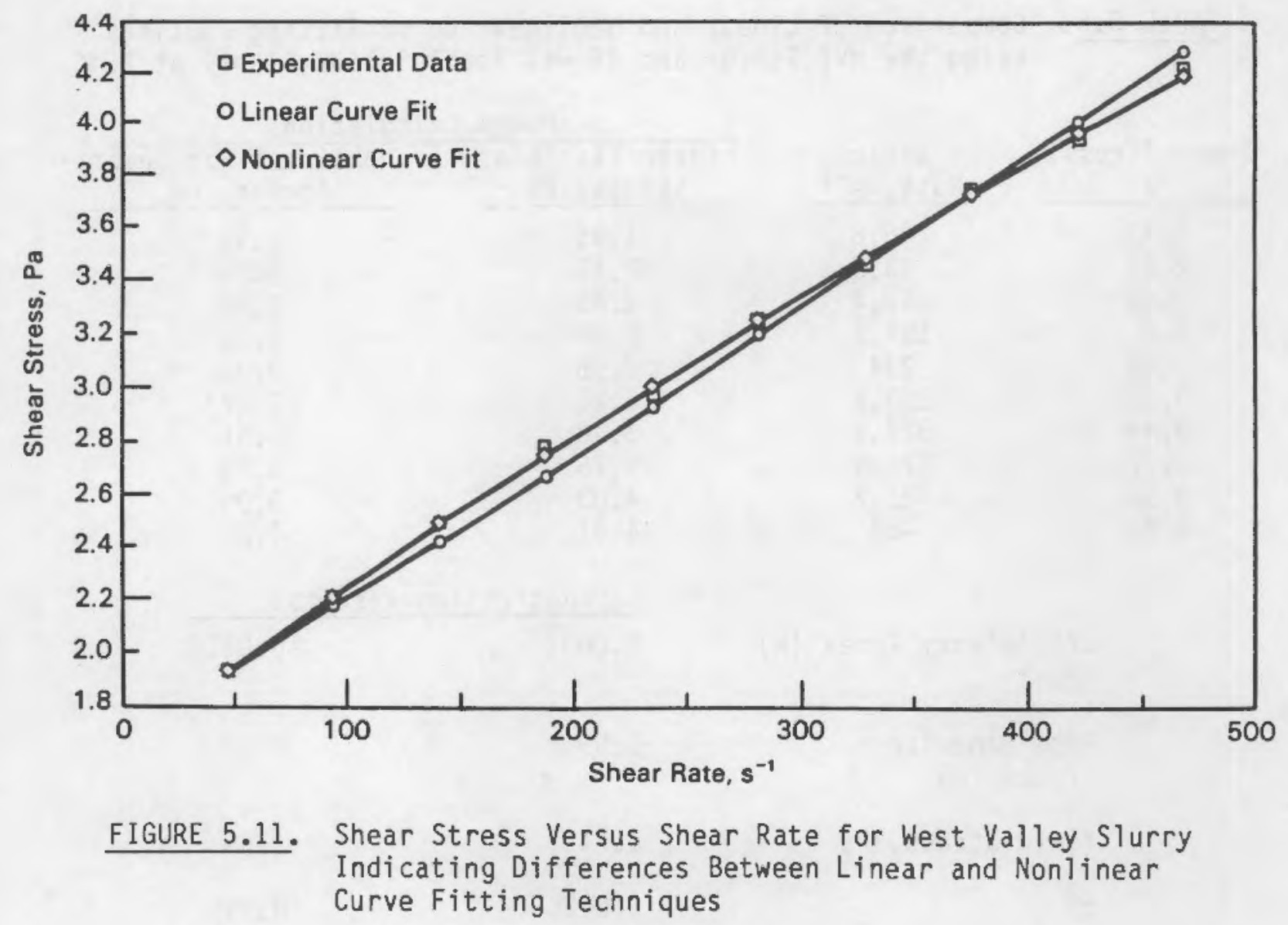

figures. The data was fit to the yield-power law model using a linear curvefitting technique. The yield-power law model which fits the data is shown on the figures. The $36 \mathrm{wt} \%$ solids concentration could be classified as a yield pseudoplastic or a Bingham plastic fluid.

The results tabulated in Tables 5.4 and 5.5 and mentioned above show different rheological parameters for the various gap sizes and calculation methods. The MVI results are about the same regardless of correction for gap size. The Reiner-Rivlin equation, however, predicts different rheological constants for the different gap sizes. The consistency index appears to increase with gap size while the yield stress decreases. These trends self-compensate to a certain extent in the predictions of bob stress as a function of rotational speed as depicted in Figure 5.14. However, the parameters for the larger gap sizes do not fare so well in predicting the shear stress as a function of shear rate (Figure 5.15). 
TABLE 5.5. Comparison of Linear and Nonlinear Curve Fitting Routines Using the MVI Sensor and $36 \mathrm{wt} \%$ Total Solids Slurry at $22^{\circ} \mathrm{C}$

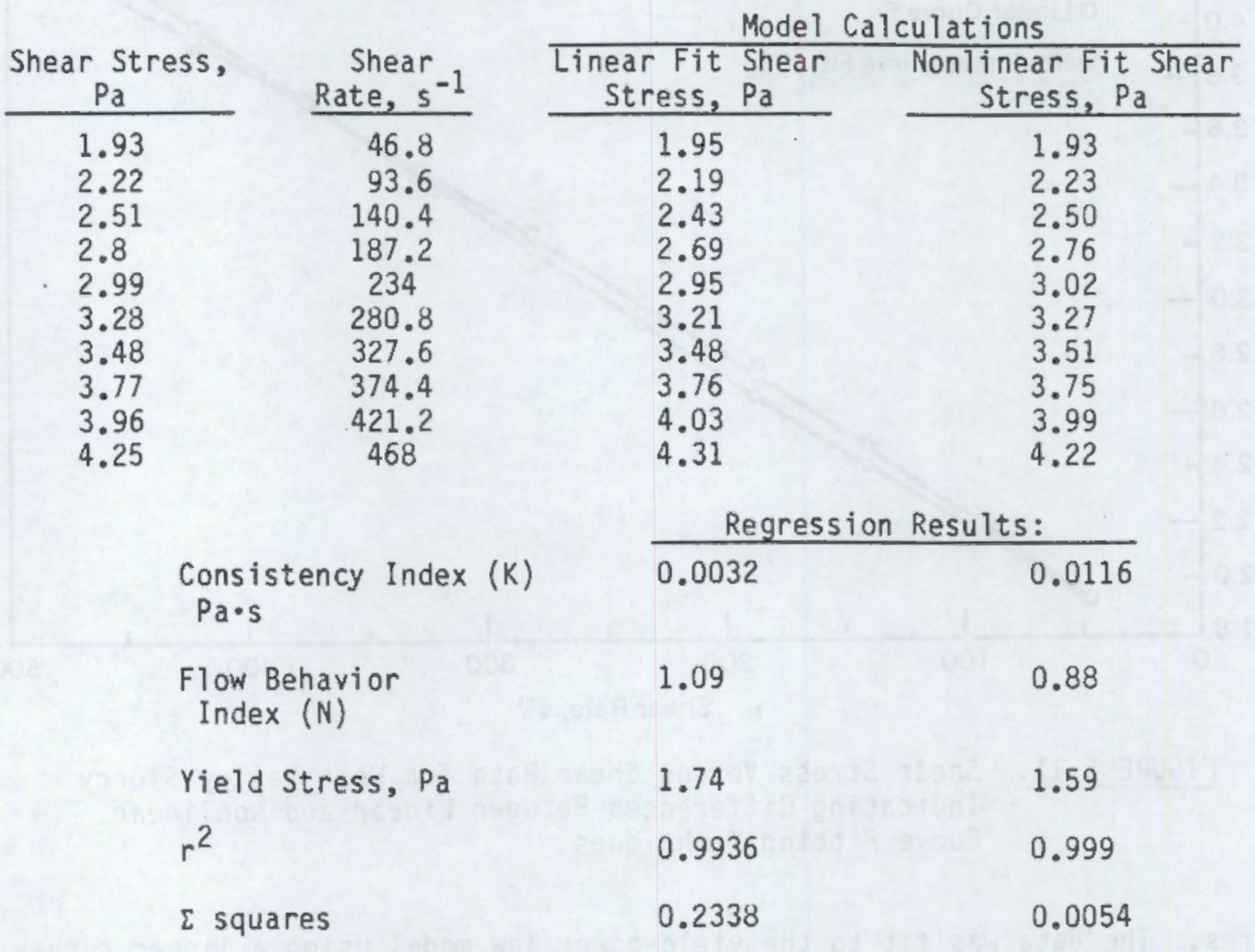

In Figure 5.9, the cylinder stress falls below the calculated yield stress for a substantial range of shear rates for the MVIII sensor and for some of the shear rates for the MVII sensor. Therefore, there would be unsheared regions under these conditions. The bob would break loose at a lower shear rate (resulting in a lower yield stress) and slurry would appear to have a higher consistency than if the whole region were sheared. The observed trends agree with this behavior. Based on these results, the MVII and MVIII data should be used only between the value where the shear rate causes the cylinder wall stress to fall below the yield stress and the value where Taylor vortices form. This region is quite narrow for the MVIII sensor. 


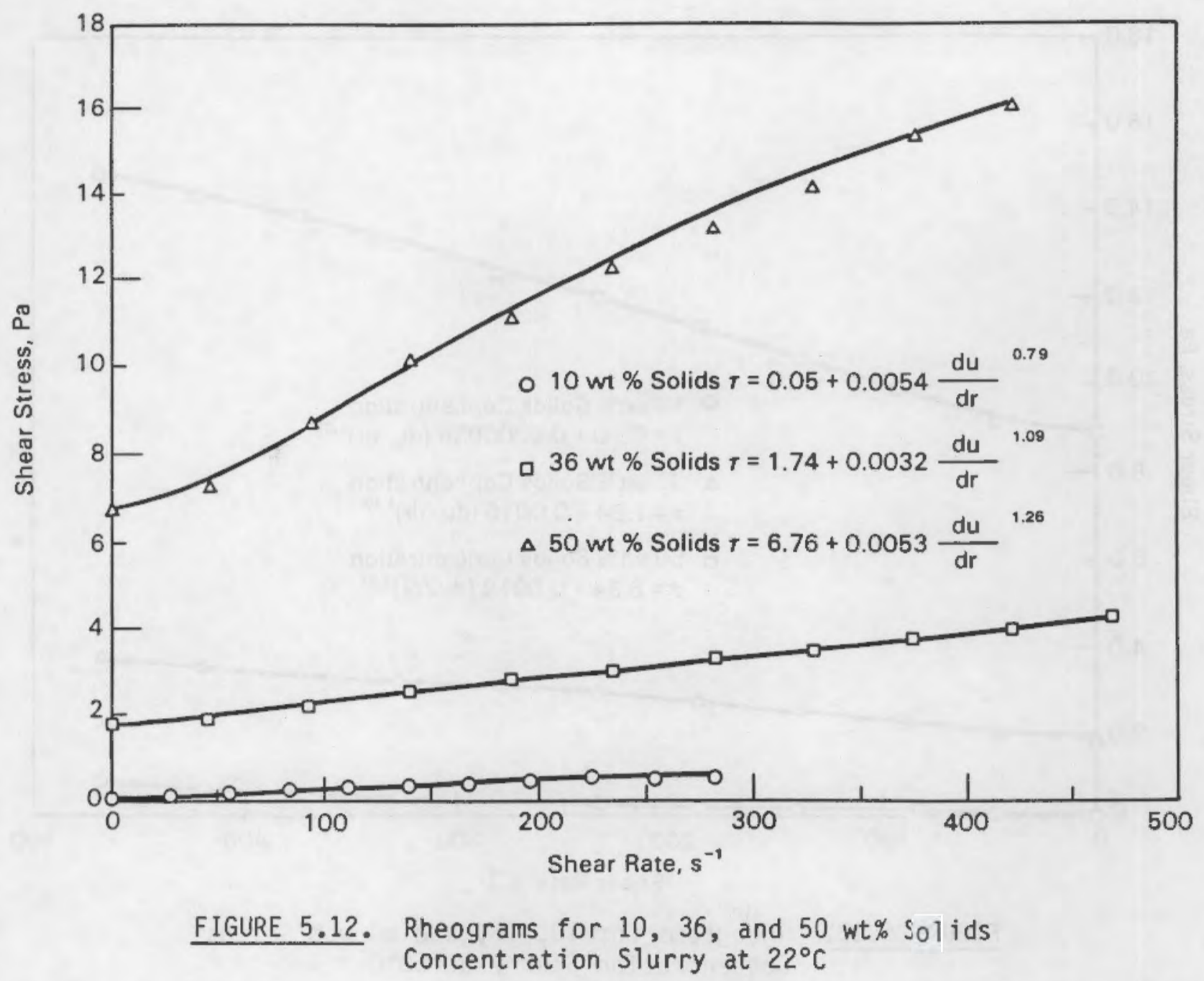

As a consequence of these problems, the MVI sensor is recommended for the determination of slurry properties. The gap size is small enough that correction for it is not required. In addition, it is small enough that Taylor vortices do not form.

The apparent viscosity was calculated for this data as a function of shear rate and temperature. Plots of the apparent viscosity are shown in Figures 5.16 and 5.17 .

To evaluate the resuspension of the bed of settled solids, the yield stress of the settled solids was measured using the Haake viscometer. The slurry was allowed to settle in the gap between the rotor and the cup for $20 \mathrm{~h}$ and $44 \mathrm{~h}$. The yield stress of the settled solids was then measured. The 


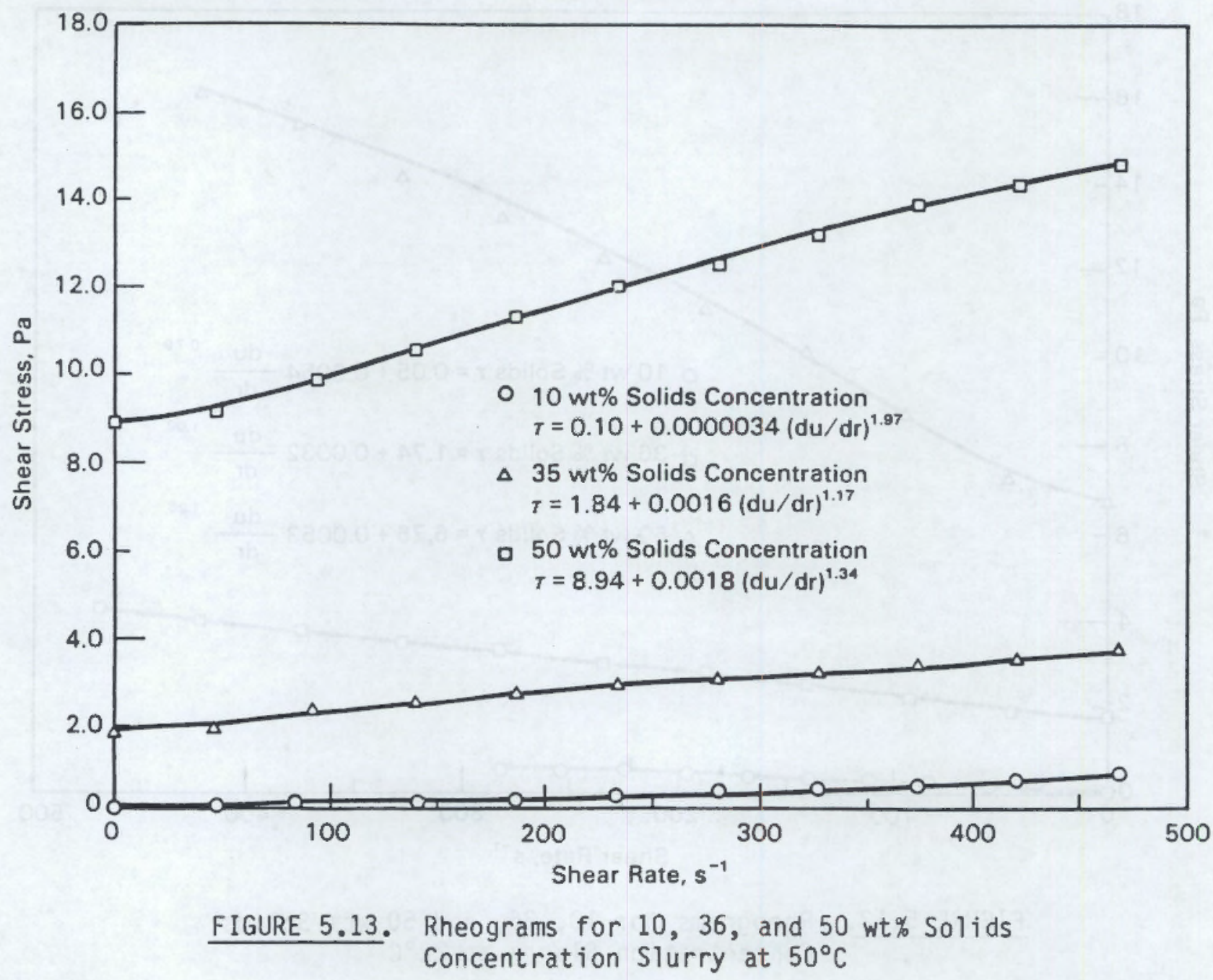

measured yield stresses are shown in Table 5.6 at both $22^{\circ} \mathrm{C}$ and $50^{\circ} \mathrm{C}$. Due to the high solids content in contact with the rotor, the MVII sensor system was used for each slurry concentration. The $20-h$ and $44-h$ settling times correspond to the settling times that were used in the pipe loop.

The rheological behavior of the supernate was determined and the data fit to the power law fluid model. The supernate was obtained by allowing the slurry to settle and removing the liquid phase. The rheological parameters for the supernate are listed in Table 5.7. The viscosity was calculated for the Newtonian behavior $(n=1)$. Rheological constants unadjusted for gap size for all sensors and different solids concentrations and sensors are depicted in Table 5.8 . 


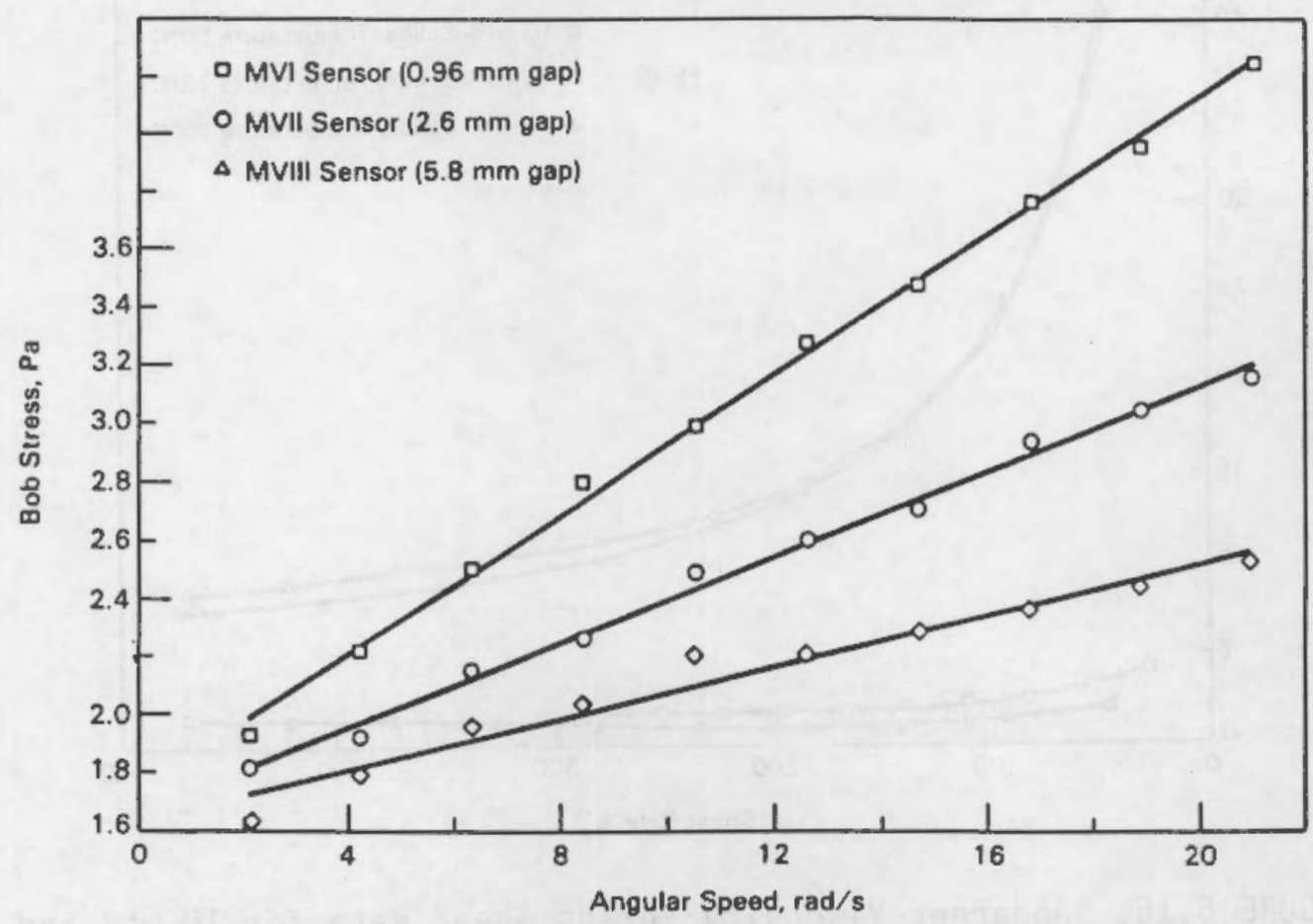

FIGURE 5.14. Bob Stress Versus Angular Speed for WV-205 Slurry Using Three Different Sensors

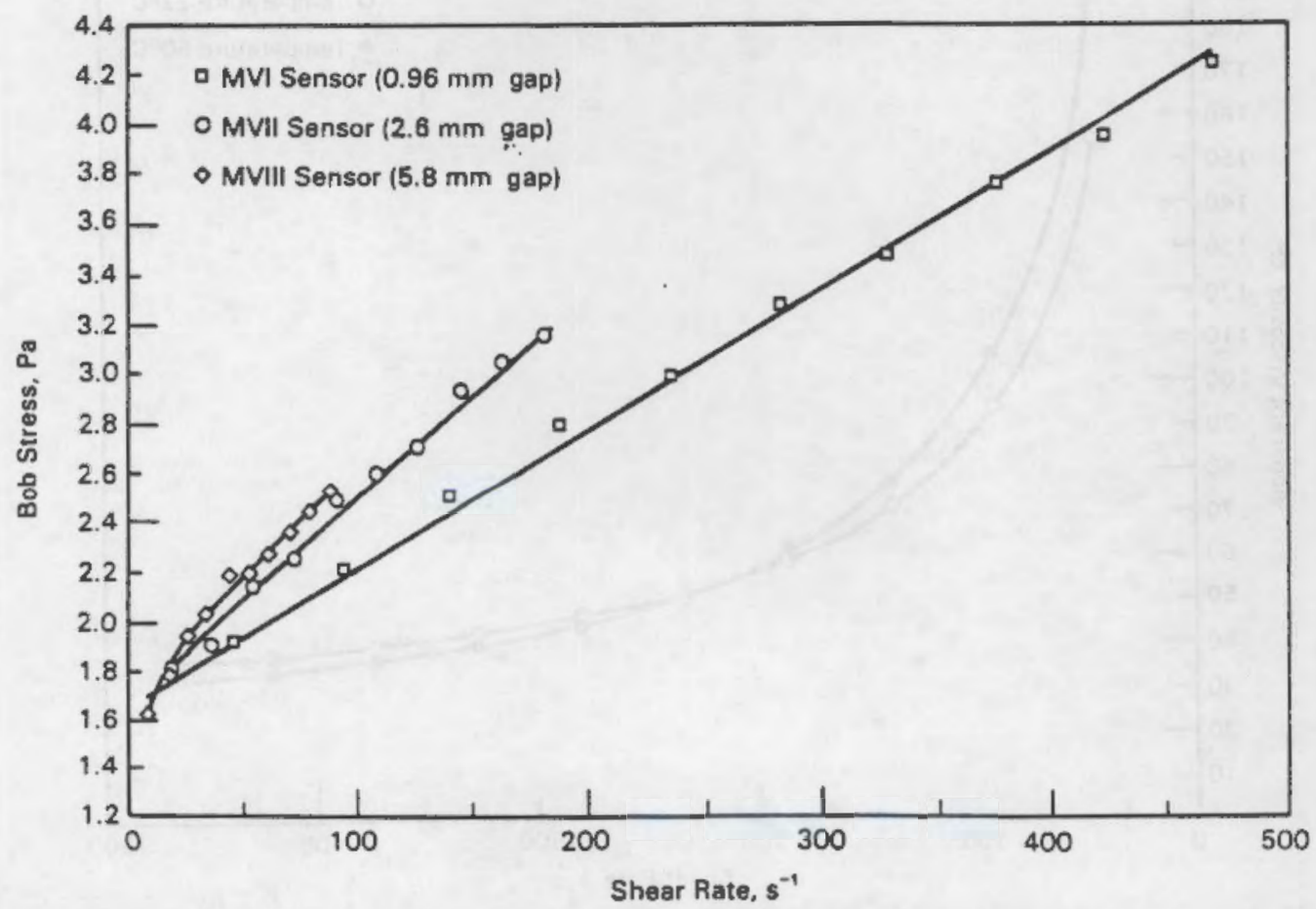

FIGURE 5.15. Bob Stress Versus Shear Rate for WV-205 Slurry Using Three Different Sensors 


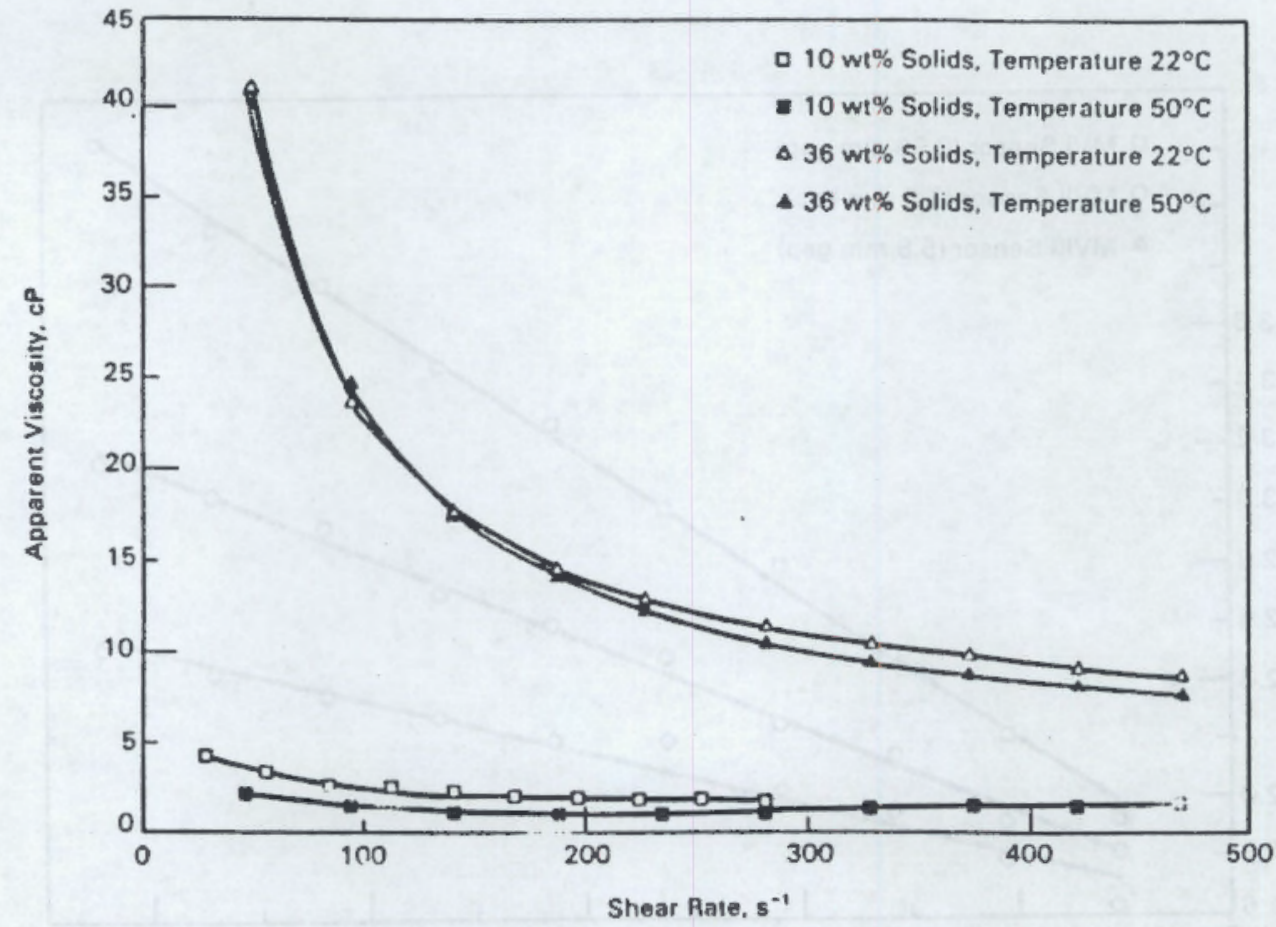

FIGURE 5.16. Apparent Viscosity Versus Shear Rate for $10 \mathrm{wt} \%$ and 36 wt\% Solids Concentration Slurry at $22^{\circ} \mathrm{C}$ and $50^{\circ} \mathrm{C}$

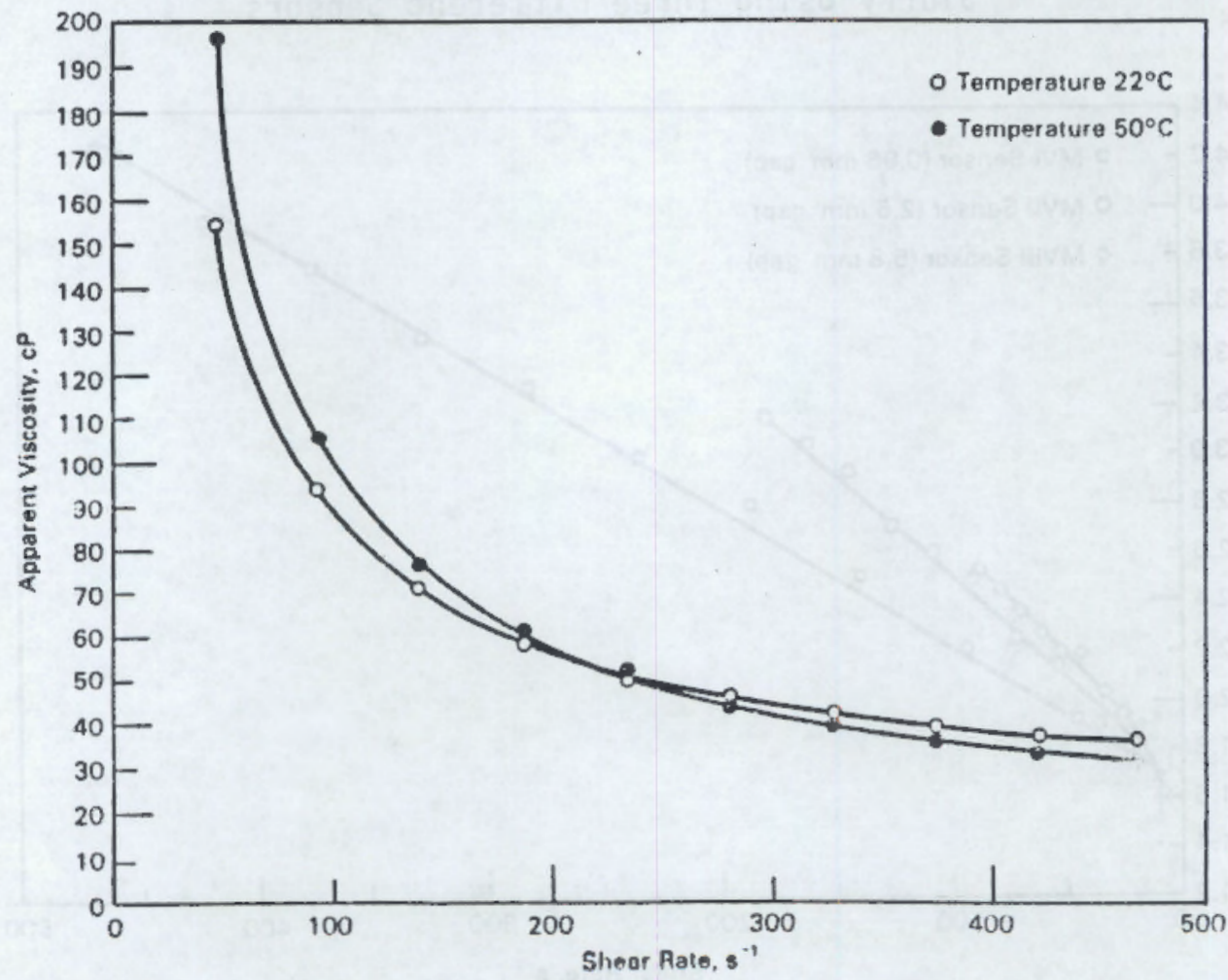

FIGURE 5.17. Apparent Viscosity Versus Shear Rate for 50 wt\% Solids Concentration Slurry at $22^{\circ} \mathrm{C}$ and $50^{\circ} \mathrm{C}$ 
TABLE 5.6. Yield Stress for Slurry Allowed to Settle for $20 \mathrm{~h}$ and $44 \mathrm{~h}$ in the Haake Viscometer

\begin{tabular}{|c|c|c|c|}
\hline $\begin{array}{l}\text { Solids } \\
\text { Concentration, wt\% }\end{array}$ & $\begin{array}{l}\text { Settling } \\
\text { Time, } h \\
\end{array}$ & $\begin{array}{c}\text { Temperature, } \\
{ }^{\circ} \mathrm{C}\end{array}$ & $\begin{array}{l}\text { Yield } \\
\text { Stress, Pa } \\
\end{array}$ \\
\hline 36 & $\begin{array}{l}20 \\
20 \\
44 \\
44\end{array}$ & $\begin{array}{l}22 \\
50 \\
22 \\
50\end{array}$ & $\begin{array}{l}3.38 \\
3.79 \\
6.10 \\
4.18\end{array}$ \\
\hline 50 & $\begin{array}{l}20 \\
44\end{array}$ & $\begin{array}{l}22 \\
50\end{array}$ & $\begin{array}{l}11.30 \\
16.67\end{array}$ \\
\hline
\end{tabular}

TABLE 5.7. Rheological Parameters for the Slurry Supernate

\begin{tabular}{|c|c|c|c|c|c|}
\hline Supernate & $\begin{array}{c}\text { Temperature, } \\
{ }^{\circ} \mathrm{C}\end{array}$ & $\begin{array}{l}\text { Consistency } \\
\text { Index } \\
(\mathrm{K}), \mathrm{Pa} \cdot \mathrm{S} \\
\end{array}$ & $\begin{array}{c}\text { Flow } \\
\text { Behavior } \\
\text { Index, } \mathrm{n} \\
\end{array}$ & $r^{2}$ & $\begin{array}{l}\text { Viscosity } \\
050 \mathrm{~s}^{-1} \\
\end{array}$ \\
\hline 10 wt\% slurry & $\begin{array}{l}22 \\
50\end{array}$ & $\begin{array}{l}0.0021 \\
0.0023\end{array}$ & $\begin{array}{l}0.92 \\
0.82\end{array}$ & $\begin{array}{l}0.9940 \\
0.9899\end{array}$ & $\begin{array}{l}1.50 \\
1.11\end{array}$ \\
\hline 36 wt\% slurry & $\begin{array}{l}22 \\
50\end{array}$ & $\begin{array}{l}0.0016 \\
0.0014\end{array}$ & $\begin{array}{l}1.00 \\
0.93\end{array}$ & $\begin{array}{l}1.0000 \\
0.9951\end{array}$ & $\begin{array}{l}1.65 \\
1.09\end{array}$ \\
\hline 50 wt\% slurry & $\begin{array}{l}22 \\
50\end{array}$ & $\begin{array}{l}0.0030 \\
0.0020\end{array}$ & $\begin{array}{l}0.99 \\
0.93\end{array}$ & $\begin{array}{l}0.9977 \\
0.9937\end{array}$ & $\begin{array}{l}2.96 \\
1.51\end{array}$ \\
\hline
\end{tabular}


TABLE 5.8. Summary of Rheological Parameters for WV-205 Slurry

\begin{tabular}{|c|c|c|c|c|c|c|c|}
\hline $\begin{array}{l}\text { Solids } \\
\text { Conc., } \\
\text { wt\% }\end{array}$ & Sensor & $\begin{array}{c}\text { Gap } \\
\text { Distance, } \\
\text { mm } \\
\end{array}$ & $\begin{array}{l}\text { Temp., } \\
{ }^{\circ} \mathrm{C} \\
\end{array}$ & $\begin{array}{c}\text { Consistency } \\
\text { Index } \\
(\mathrm{K}), \mathrm{Pa} \cdot \mathrm{s} \\
\end{array}$ & $\begin{array}{l}\text { Flow } \\
\text { Behavior } \\
\text { Index, } \mathrm{n} \\
\end{array}$ & $\begin{array}{l}\text { Yield } \\
\text { Stress, } \\
\tau_{y} \mathrm{~Pa} \\
\end{array}$ & $r^{2}$ \\
\hline 10 & MVI & 0.96 & $\begin{array}{l}22 \\
50\end{array}$ & $\begin{array}{l}0.0054 \\
0.0000034\end{array}$ & $\begin{array}{l}0.79 \\
1.98\end{array}$ & $\begin{array}{l}0.05 \\
0.10\end{array}$ & $\begin{array}{l}0.9913 \\
0.9589\end{array}$ \\
\hline 10 & MVII & 2.6 & $\begin{array}{l}22 \\
50\end{array}$ & $\begin{array}{l}0.00032 \\
0.0069\end{array}$ & $\begin{array}{l}1.54 \\
1.36\end{array}$ & $\begin{array}{l}0.17 \\
0.18\end{array}$ & $\begin{array}{l}0.9968 \\
0.9888\end{array}$ \\
\hline 10 & MVIII & 5.8 & $\begin{array}{l}22 \\
50\end{array}$ & $\begin{array}{l}0.0011 \\
0.0081\end{array}$ & $\begin{array}{l}1.43 \\
1.00\end{array}$ & $\begin{array}{l}0.16 \\
0.16\end{array}$ & $\begin{array}{l}0.9981 \\
0.9693\end{array}$ \\
\hline 36 & MVI & 0.96 & $\begin{array}{l}22 \\
50\end{array}$ & $\begin{array}{l}0.0032 \\
0.0016\end{array}$ & $\begin{array}{l}1.09 \\
1.17\end{array}$ & $\begin{array}{l}1.74 \\
1.84\end{array}$ & $\begin{array}{l}0.9933 \\
0.9264\end{array}$ \\
\hline 36 & MVII & 2.6 & $\begin{array}{l}22 \\
50\end{array}$ & $\begin{array}{l}0.0041 \\
0.0079\end{array}$ & $\begin{array}{l}1.15 \\
0.93\end{array}$ & $\begin{array}{l}1.70 \\
1.92\end{array}$ & $\begin{array}{l}0.9918 \\
0.9935\end{array}$ \\
\hline 36 & MVIII & 5.8 & $\begin{array}{l}22 \\
50\end{array}$ & $\begin{array}{l}0.1161 \\
0.1381\end{array}$ & $\begin{array}{l}0.99 \\
0.80\end{array}$ & $\begin{array}{l}1.63 \\
1.79\end{array}$ & $\begin{array}{l}0.9395 \\
0.8590\end{array}$ \\
\hline 50 & MVI & 0.96 & $\begin{array}{l}22 \\
50\end{array}$ & $\begin{array}{l}0.0053 \\
0.0018\end{array}$ & $\begin{array}{l}1.26 \\
1.34\end{array}$ & $\begin{array}{l}6.76 \\
8.94\end{array}$ & $\begin{array}{l}0.9692 \\
0.9754\end{array}$ \\
\hline 50 & MVI I & 2.6 & $\begin{array}{l}22 \\
50\end{array}$ & $\begin{array}{l}0.3735 \\
0.0048\end{array}$ & $\begin{array}{l}0.96 \\
1.20\end{array}$ & $\begin{array}{l}7.06 \\
9.61\end{array}$ & $\begin{array}{l}0.9993 \\
0.9903\end{array}$ \\
\hline 50 & MVIII & 5.8 & $\begin{array}{l}22 \\
50\end{array}$ & $\begin{array}{l}0.1517 \\
0.0598\end{array}$ & $\begin{array}{l}0.75 \\
0.85\end{array}$ & $\begin{array}{l}6.52 \\
8.79\end{array}$ & $\begin{array}{l}0.9964 \\
0.9938\end{array}$ \\
\hline
\end{tabular}




\subsection{PILOT-SCALE TRANSPORT OF HOMOGENEOUS SLURRY}

The transport characteristics of fully homogeneous WV-205 slurry were determined prior to the resuspension experiments. Section 6.1 describes the experimental apparatus, including the capillary viscometer and the pipe loop. Section 6.2 describes the calibration of the apparatus. Section 6.3 provides the results of the experiments with a Newtonian fluid. Section 6.4 provides the results of the experiments using WV-205 slurry.

\subsection{DESCRIPTION OF EXPERIMENTAL APPARATUS}

Several experimental systems were constructed at the University of Idaho for the evaluation of the rheological properties and resuspension of the WV-205 slurry. A laboratory-scale capillary viscometer was constructed for determining the rheological properties of the slurry. A pilot-scale pipe loop apparatus was constructed for evaluating transport and resuspension characteristics of the slurry and for determining the rheological properties. These properties were compared to the rheological properties determined using a rotational viscometer. A laboratory-scale resuspension apparatus was also constructed to determine resuspension in the laboratory.

\subsubsection{Capillary Viscometer}

The capillary viscometer (Figure 6.1) was constructed of lengths of stainless steel tubing $0.9208,0.9152,0.9168$, and $0.92 \mathrm{~m}$ long. The respective inside diameters of the tubing were $2.39,3.4,4.57$, and $5.33 \mathrm{~mm}$. Flow through the tubes was produced by nitrogen pressure to a mixed tank which contained the slurry. Four differential pressure transducers, sized for 10-in. water, 1-psi, 5-psi, and 30-psi pressure differentials, were used depending on the expected pressure drops. The computer data acquisition system, discussed in Section 6.1.2, was used to record the pressure drop for a given flow rate. The calibration runs were conducted with a 42 wt\% sugar solution. Data from the calibration runs are presented in Section 6.2. 


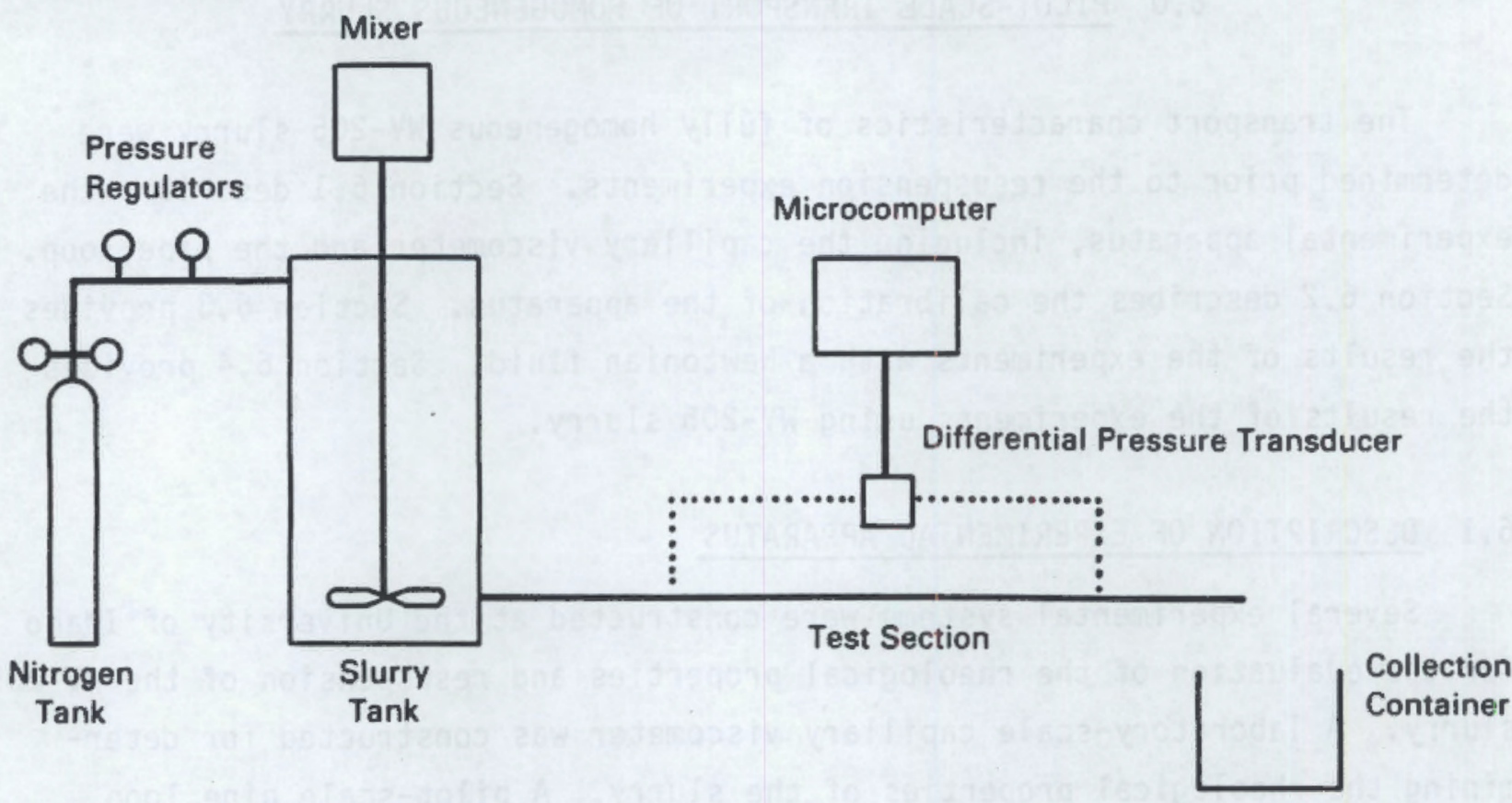

FIGURE 6.1. Schematic Diagram of Capillary Viscometer

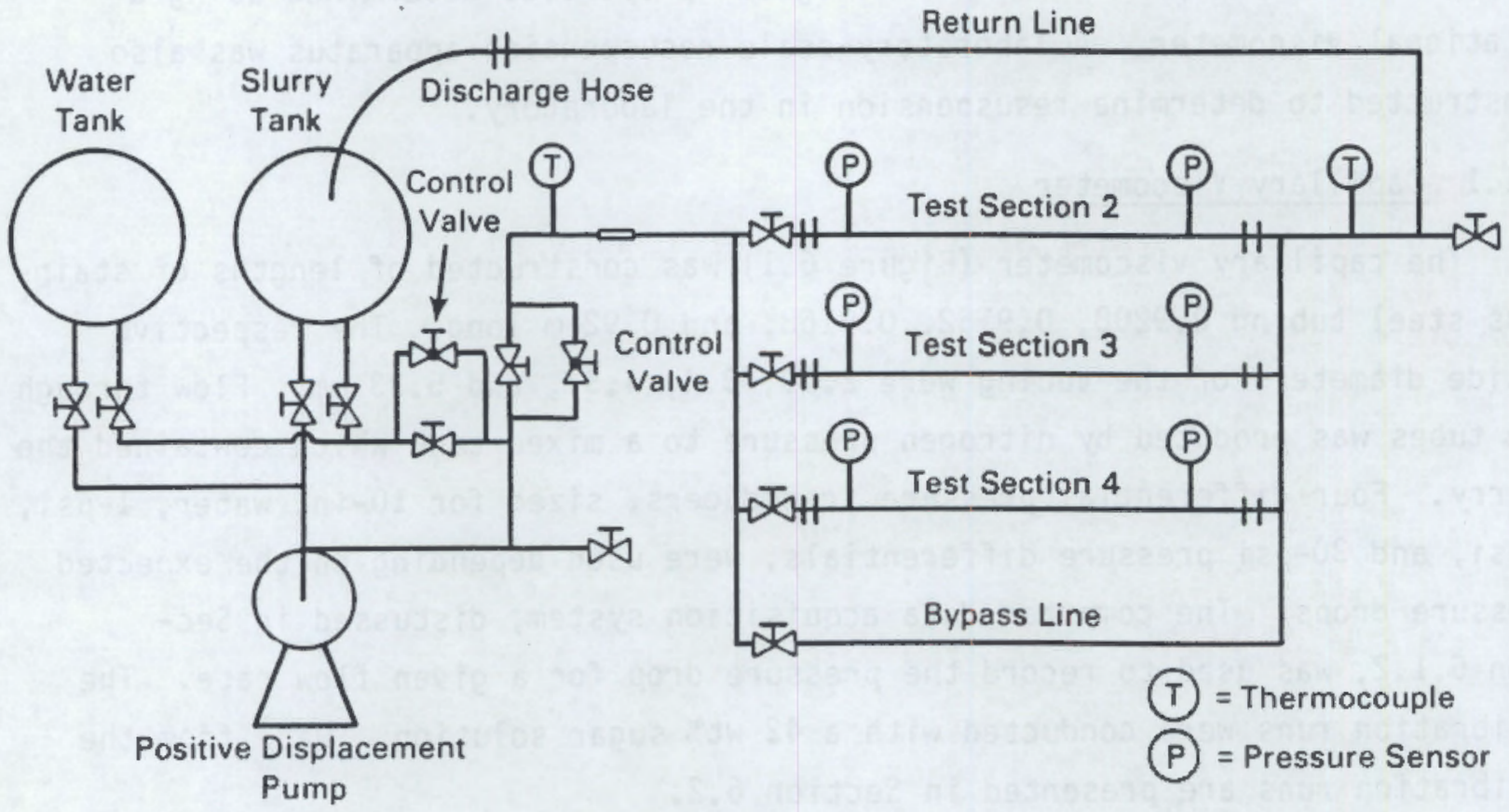

FIGURE 6.2. Schematic Diagram of Pipe Flow Test Loop 


\subsubsection{Pipe Loop Apparatus}

The flow test loop was constructed at the University of Idaho. A schematic diagram is shown in Figure 6.2. The system consists of a 250-gal slurry feed tank with an agitator, pump, venturi flow meter, differential pressure transducers, two data acquisition boards to allow interface of the transducer output with the computer, a personal computer, and interchangeable 24-ft test sections of clear pipe. Three different test sections of $1 / 4-i n .(6.35-\mathrm{mm})$, $3 / 8-i n .(9.52-\mathrm{mm})$, and 1/2-in. (12.7-mm) ID were used.

A positive displacement slurry pump was chosen for slurry pumping due to the reduced shearing of particles from its action as well as its ability to pump slurries with high solids concentrations.

The data acquisition system consists of a microcomputer with data acquisition interfaces, four differential pressure transducers at pressures of 10 inches of water, 1 psi, 5 psi, and 30 psi, type $K$ thermocouples, and a pumpshaft tachometer. Flow rates for the water tests were measured with a venturi meter which was calibrated with timed volumes of water, i.e., the "bucket and stopwatch" method for flowmeter calibration. For the slurry runs and sugar calibration work, timed volumes were used to determine the flow rate. An attempt was made to use the pump speed as an additional indication of the flow rate. This was not a good method since the pump was oversized for the low flow rates desired. A portion of the flow bypassed the pump; consequently pump speed measurements did not allow determination of flow through the pipe loop. Tests were conducted at room temperature $\left(20\right.$ to $\left.27^{\circ} \mathrm{C}\right)$ and fluid temperatures were monitored with a glass thermometer and thermocouple. Silicone oil was used in the lines from the pressure taps on the test sections to the pressure transducers to prevent slurry from entering and plugging these lines. Rigid tubing of $3.17-\mathrm{mm}$ ID was required for the pressure lines because flexible tubing deformed at the higher pressures. The pressure lines contained a tee to remove air bubbles in the lines and to refill the lines with silicone oil. The pressure transducers were calibrated under static conditions with water and mercury manometers.

Data was collected within the range of the equipment and instrumentation capabilities. The two limiting factors were the pressure transducers which 
have a maximum span of $30 \mathrm{psi}$ and the pump output which is approximately $10 \mathrm{gpm}$. A minimum of 25 data points spanning laminar, transition, and turbulent flow were taken during steady-state flow operation. Each data point consisted of a differential pressure reading over a straight horizontal length of tube. The computer acquisition system was used to obtain averages for the pressure drops. These averages were the average of 15 readings taken at one reading/sec. The test was repeated with flow conditions selected to replicate flow regions of particular interest.

This system was calibrated with water for the turbulent regime and a sugar-water solution for the laminar regime. The sugar-water solution was prepared by dissolving the contents of 25-1b (nominal weight) bags of sugar in tap water. Twenty bags of sugar were dissolved in 500 lb of water. Subsequent measurements of the viscosity and density indicated that the sugar concentration was approximately $60 \mathrm{wt} \%$.

\subsection{CALIBRATION OF APPARATUS}

The transducers used in the test program were calibrated with water and mercury manometers. The pressure drop across the transducer and the corresponding output voltage were determined for each transducer ( $10 \mathrm{in} . \mathrm{H}_{2} \mathrm{O}, 1$ psi, 5 psi, and $30 \mathrm{psi}$ ). The calibration of the capillary viscometer and pipe loop are discussed in Section 6.3 .

\subsection{NEWTONIAN FLUID EXPER IMENTS}

Calibration of the laboratory-scale capillary tube viscometer was conducted by means of flow experiments. Pressure drop was measured as a function of flow rate. All experiments were performed at room temperature.

The capillary tubes were calibrated using a 42 wt\% sugar-water solution at $27^{\circ} \mathrm{C}$. The shear stress versus shear rate for the different sized capillary tubes is plotted in Figure 6.3. The linear fit is good with a $r^{2}$ of 0.9995 . The plot has a slope of $0.0056 \mathrm{~Pa} \cdot \mathrm{s}$ (corresponding to a viscosity of $5.6 \mathrm{CP}$ ). 


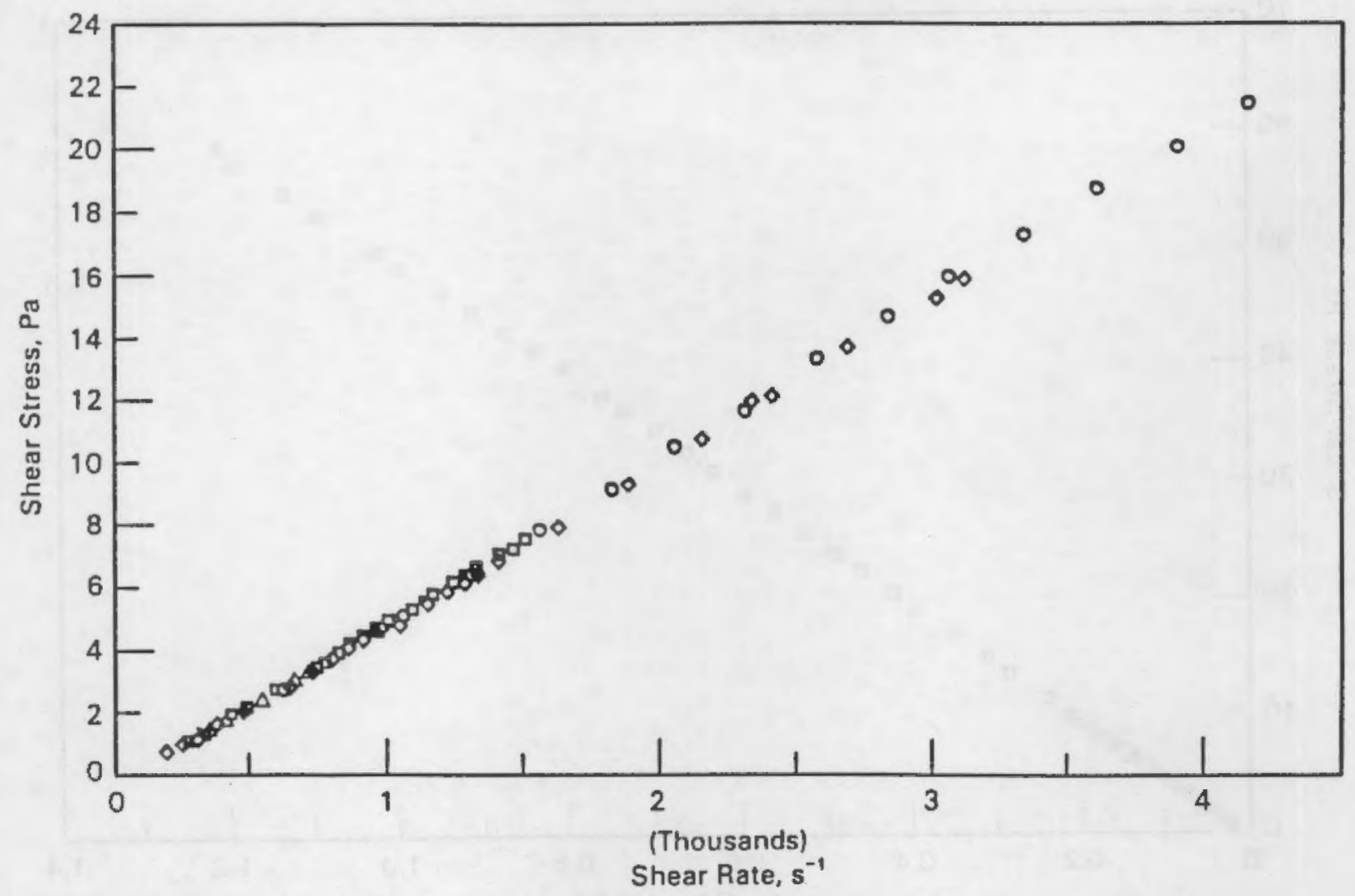

FIGURE 6.3. Shear Stress Versus Shear Rate for a 42 wt\% Sugar Solution Using the Capillary Tube Viscometer with All Four Tube Diameters

These results are about equal to those obtained with a Cannon Fenske viscometer $\left(5.68 \mathrm{CP}\right.$ at $\left.26.7^{\circ} \mathrm{C}\right)$ and comparable to those tabulated $\left(6.014 \mathrm{CP}\right.$ at $25^{\circ} \mathrm{C}$ and $6.234 \mathrm{CP}$ at $24^{\circ} \mathrm{C}$--Hodgman 1960 ).

The pilot test loop was calibrated using water and a sugar solution. Due to the insensitivity of the pressure transducers at the low range, experiments with water were conducted to check the results against correlations for turbulent flow and experiments with 60 wt\% sugar solution were conducted to check the operation of the apparatus in laminar flow.

A linear plot of the shear stress versus shear rate for the $6.35-\mathrm{mm}-\mathrm{ID}$ pipe is depicted in Figure 6.4. A yield power law model fit to these results indicates a yield stress of $-0.423 \mathrm{~Pa}$, a consistency index of $0.0557 \mathrm{~Pa} \cdot \mathrm{s}$, and a flow behavior index of 0.983 with an $r^{2}$ of better than 0.999 . A Newtonian 


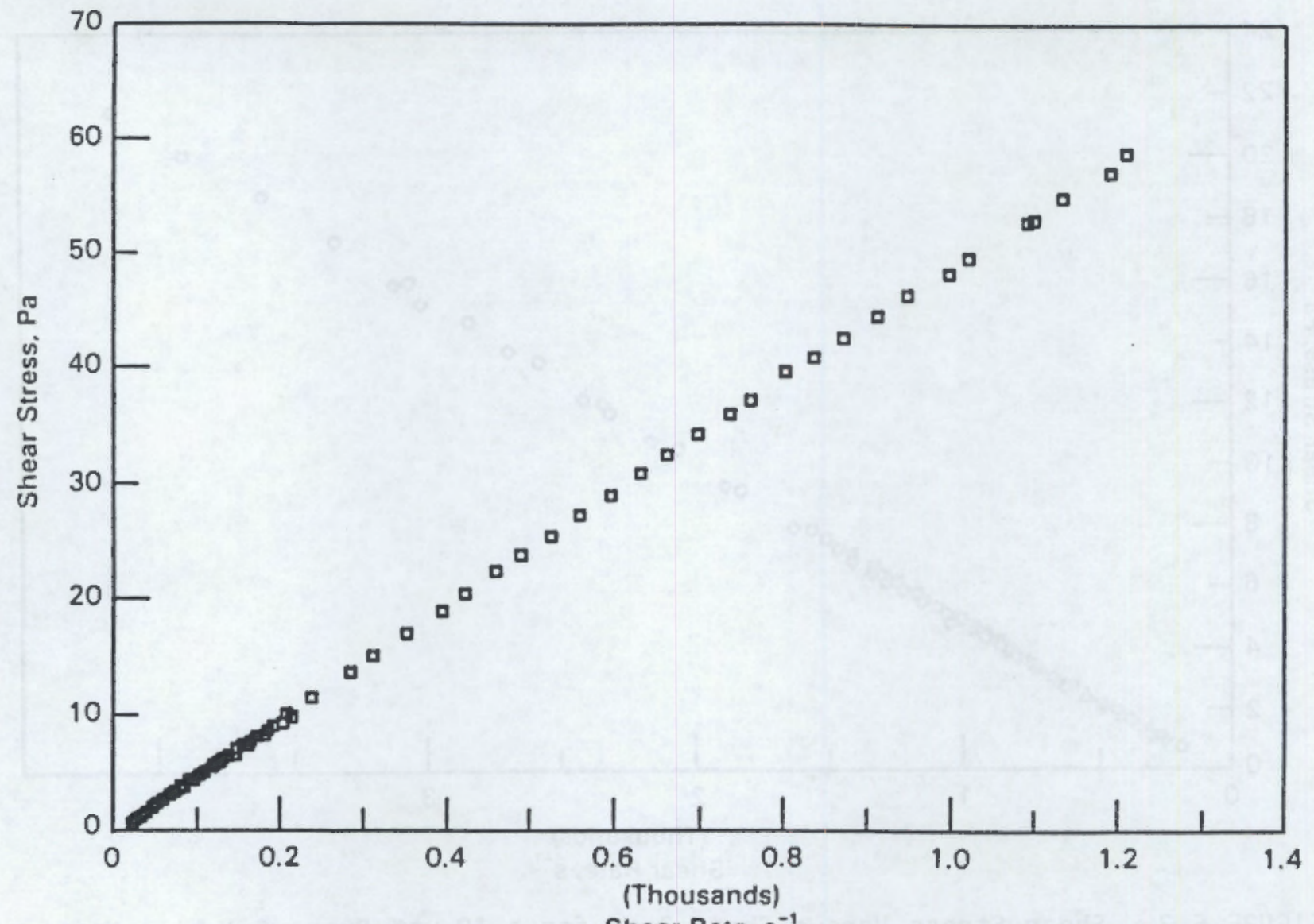

Shear Rate, $\mathrm{s}^{-1}$

FIGURE 6.4. Pipe-Loop Calibrations of Shear Stress Versus Shear Rate Using a $60 \mathrm{wt} \%$ Sugar-Water Solution in the 6.35-mm-ID Pipe

model fit to these data indicates a viscosity of $0.049 \mathrm{~Pa} \cdot \mathrm{s}$ and an intercept of $-0.224 \mathrm{~Pa}$. The low negative yield stresses are within experimental error to indicate the fluid has no yield stress. These results agree fairly well with the viscosity as measured by a Cannon Fenske viscometer and tabulated values for a $60 \mathrm{wt} \%$ sugar solution. Table 6.1 summarizes the results for the three pipe sizes and includes literature values for the sugar viscosity at the various experimental temperatures $\left(52 \mathrm{CP}\right.$ at $22^{\circ} \mathrm{C}$ ) as well as viscosity determination by means of a Cannon Fenske viscometer. The viscosity as determined by this device is $58 \mathrm{CP}$ at $22^{\circ} \mathrm{C}, 47 \mathrm{CP}$ at $27^{\circ} \mathrm{C}$, and $43 \mathrm{CP}$ at $29^{\circ} \mathrm{C}$.

The sugar-water tests yielded good correlations between the actual data and the expected behavior of a Newtonian fluid of high viscosity (relative to water). The differences in viscosity for the different pipe sizes can probably 
TABLE 6.1. Rheological Constants for 60 wt\% Sugar Water Runs in the Pipe Loop

\begin{tabular}{|c|c|c|c|}
\hline \multirow[b]{2}{*}{ Parameter } & & & \\
\hline & 6.35 & 9.52 & 12.7 \\
\hline $\begin{array}{l}\text { Slope of } \log \text { stress } \\
\text { versus } \log \text { shear rate }\end{array}$ & 0.959 & 1.006 & 1.010 \\
\hline $\begin{array}{l}\text { Intercept of } \log \\
\text { stress versus } \log \text { shear }\end{array}$ & 3.158 & 3.494 & 3.358 \\
\hline Yield Pseudoplastic & & & \\
\hline Yield Stress, Pa & -0.422 & 0.0347 & 0.324 \\
\hline Consistency Index, Pa.s & 0.0557 & 0.0296 & 0.0328 \\
\hline Flow Behavior index & 0.983 & 1.000 & 0.999 \\
\hline$r^{2}$ & 0.9999 & 0.9999 & 0.9999 \\
\hline
\end{tabular}

$\begin{array}{lccc}\text { Newtonian } & & & \\ \text { Intercept } & -0.224 & 0.0328 & 0.0327 \\ \text { Viscosity, Pa.s } & 0.0495 & 0.0297 & 0.0336 \\ r^{2} & 0.9998 & 0.9999 & 0.9986\end{array}$

Mean of Apparent
Viscosities, $\mathrm{Pa} \cdot \mathrm{s}$
0.0467
0.0299
0.0339

Run Temperature, ${ }^{\circ} \mathrm{C}$

22

28

28
Note: Viscosity of $60 \mathrm{wt} \%$ sugar solutions$$
\frac{\mathrm{T},{ }^{\circ} \mathrm{C}}{20} \quad \frac{\text { Viscosity, Pa.S }}{0.058}
$$$$
21 \quad 0.055
$$$$
22 \quad 0.052
$$$$
23 \quad 0.049
$$$$
24 \quad 0.046
$$$$
25 \quad 0.040
$$
from Handbook of Chemistry and Physics (Hodgman 1960).


be attributed to temperature variations between runs. These data indicate that the pipe loop apparatus and associated data aquisitions will provide accurate data.

A logarithmic plot of the shear stress versus shear rate data $\left(T=24^{\circ} \mathrm{C}\right)$ for the 6.35-mm-ID pipe is depicted in Figure 6.5. The curve shows two distinct linear regions corresponding to laminar flow $(\operatorname{Re}<2600)$ and turbulent flow. Laminar flow occurred at a shear rate less than $518 \mathrm{~s}^{-1}$ and a flow rate of $0.41 \mathrm{~m} / \mathrm{s}$ in the $6.35-\mathrm{mm}-\mathrm{ID}$ pipe. For laminar flow where $f=16 / \mathrm{Re}$, the slope of the log-log plot should equal unity and the intercept should equal the $\log$ of the viscosity. For turbulent flow where $f=0.0791 \mathrm{Re}^{-1 / 4}$, the slope should equal 1.75 and the intercept should equal $\log 0.00104 \mu^{1 / 4} D^{1.5} \rho^{0.75}$.

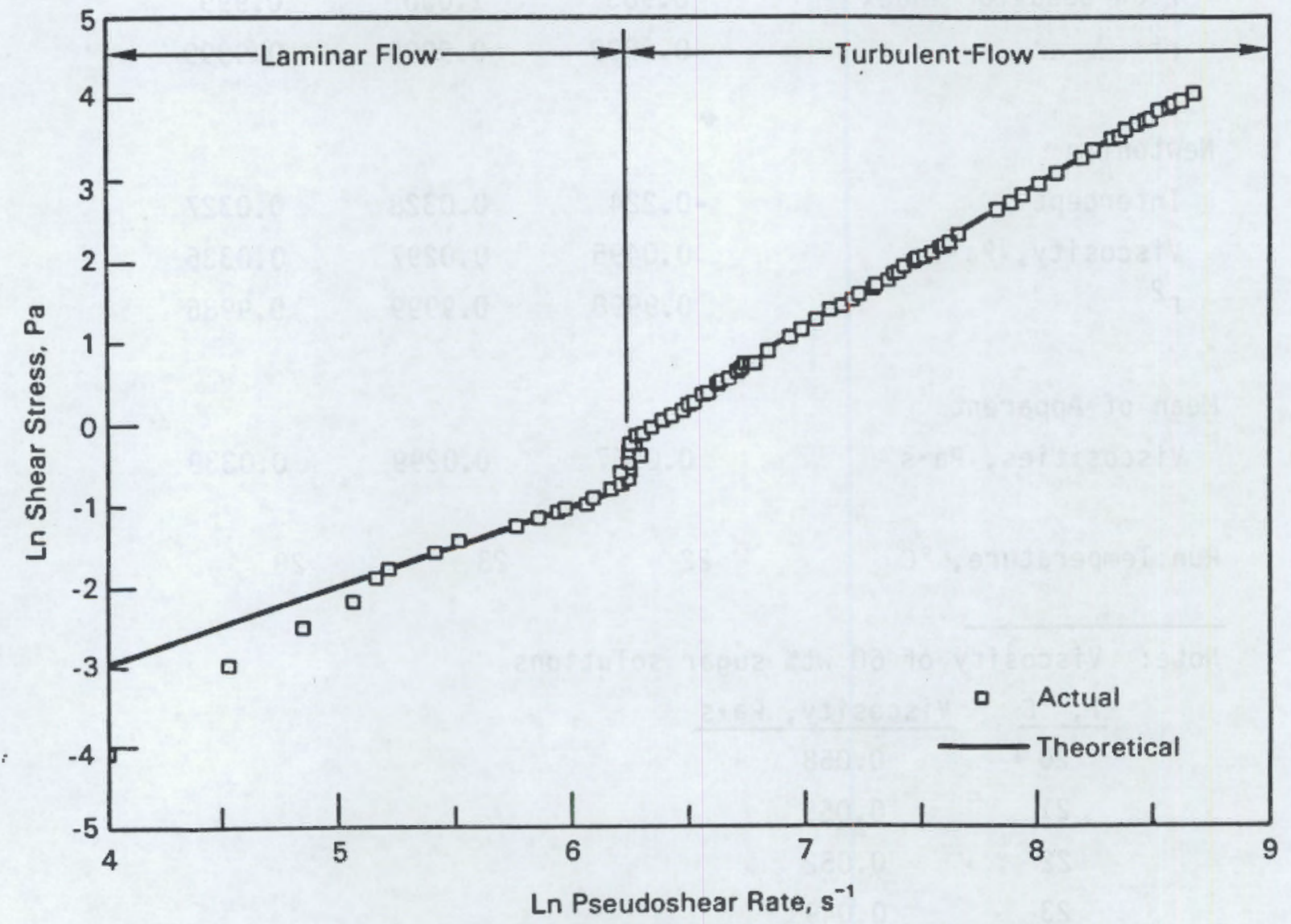

FIGURE 6.5. Logarithmic Plot of Pipe Loop Calibrations Showing Shear Stress Versus Shear Rate Using Water in the 6.35-mm-ID Pipe 
In the laminar flow region, the slope of a line fit to the data is 1.277 and the intercept is -8.607 . A 1 inear plot of the shear stress versus shear rate indicates a viscosity of $0.000998 \mathrm{~Pa} \cdot \mathrm{s}$ with an intercept of $-0.0296 \mathrm{~Pa}$ and an $r^{2}$ of 0.987 . A Mooney-Rabinowitsch correction (Section 3.2) for the shear rate results in a viscosity of $0.00105 \mathrm{~Pa} \cdot \mathrm{s}$, an intercept of $-0.296 \mathrm{~Pa}$, and an $r^{2}$ value of 0.987 . Although the $\log -\log$ plot results indicate that the fluid is non-Newtonian, the linear results and the Mooney Rabinowitsch correction indicate essentially Newtonian behavior and a viscosity of $0.001 \mathrm{~Pa} \cdot \mathrm{s}$ ( $1 \mathrm{CP}$ ).

Also depicted in Figure 6.5 are lines representing laminar and turbulent flow, based on $f=16 / R e$ for the laminar region and $f=0.0791 \mathrm{Re}^{-1 / 4}$ for the turbulent region. The agreement between the laminar flow and the correlation is poor at the low shear rates. The experimental data in this region is from measurements with the $10-i n .-\mathrm{H}_{2} \mathrm{O}$ transducer and the discrepancy may have resulted from the inaccuracies of measurements at the low pressure drops for this transducer. Data in the turbulent region are for three transducers, all in the psi range and are probably more accurate.

The agreement between the data and the correlation for flow in the turbulent region indicates that the pipe may be considered free of roughness. Figure 6.6 is a plot of friction factor versus Reynolds number for all three pipe sizes. Also on the figure are lines for the theoretical correlations for laminar and turbulent flow. The agreement between the turbulent flow data and the theoretical predictions is good, whereas the laminar flow data for water do not agree well with the predictions. The laminar flow data for sugar water, also depicted on the figure, agrees well with the laminar correlation. This good agreement is probably due to the higher accuracy and sensitivity of the sensors used for the sugar water solution experiments. The sugar water solution had a higher viscosity and higher pressure drops in the laminar region than water.

The test results summarized above indicate that the capillary and pipe loop systems will accurately determine the rheological properties of Newtonian fluids. Due to the inaccuracies of the 10-in. transducer at the low pressures, analysis of the experimental data should be limited to the higher pressure range for this transducer. The other transducers may be experiencing a similar 


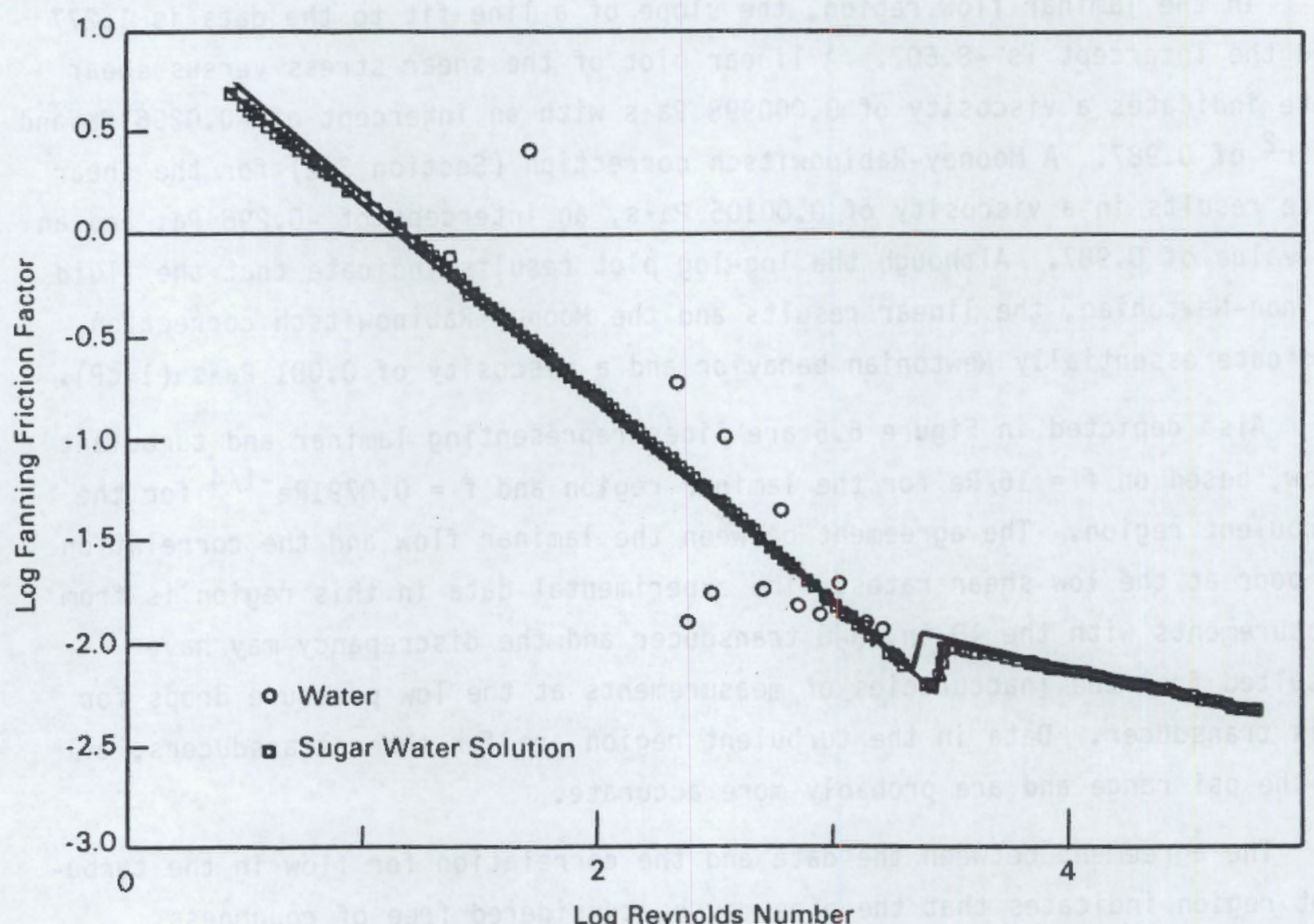

FIGURE 6.6. Fanning Friction Factor Versus Reynolds Number for All Three Pipe Loop Diameters Using Both Water and Sugar-Water Solution

problem. In the slurry work that follows, the lower differential pressure transducer measurements were eliminated when they overlapped with the measurements from the higher end of another pressure transducer.

\subsection{SLURRY TRANSPORT EXPERIMENTS}

All of the tests summarized in this section were performed at room temperature $\left(22\right.$ to $\left.29^{\circ} \mathrm{C}\right)$ with the WV-205 slurry at $36 \mathrm{wt} \%$ solids. After the slurry was delivered to the University of Idaho, some large 3- to 6-mm lumps were found settled out in the bottom of the carboys. The slurry was coarse screened to remove these lumps; the lumps were ground and then added to the slurry in a 250-gal tank. 


\subsubsection{Capillary Tube Results}

The experimental work with the capillary tubes indicated that the slurry was non-Newtonian. Figure 6.7 depicts the data of shear stress versus pseudoshear rate $(8 \mathrm{~V} / \mathrm{D})$ on a $\log -\log$ plot. Based on these data, a power series was fit to the data to develop an analytical expression for the shear stress as a function of the pseudoshear rate. This expression was used to adjust the shear rate for non-Newtonian behavior as described in Section 3.2. A plot of the shear stress versus adjusted shear rate appears in Figure 6.8. Based on these results, a nonlinear regression was performed to determine the rheological parameters for a yield pseudoplastic fluid. These parameters are tabulated in

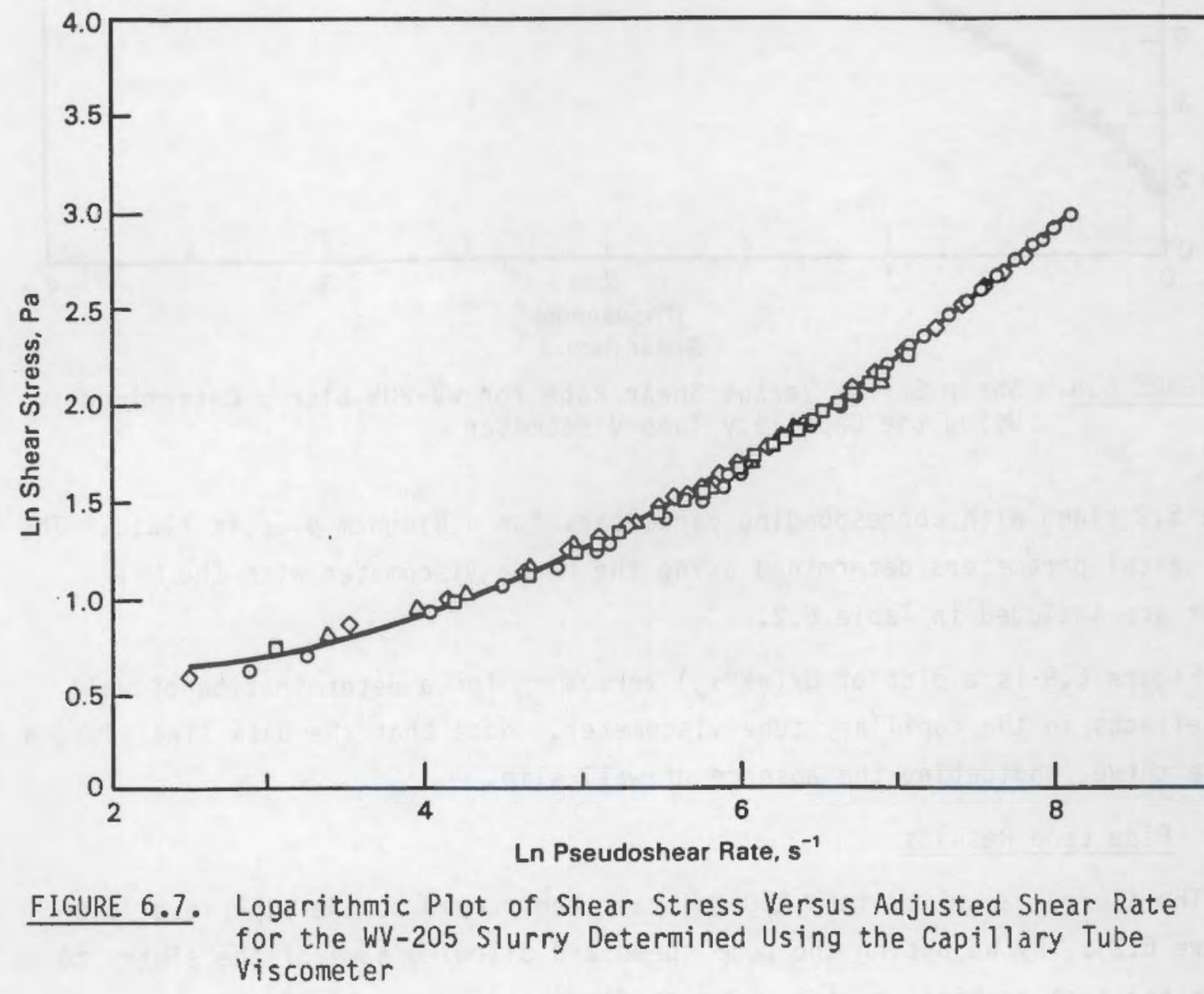




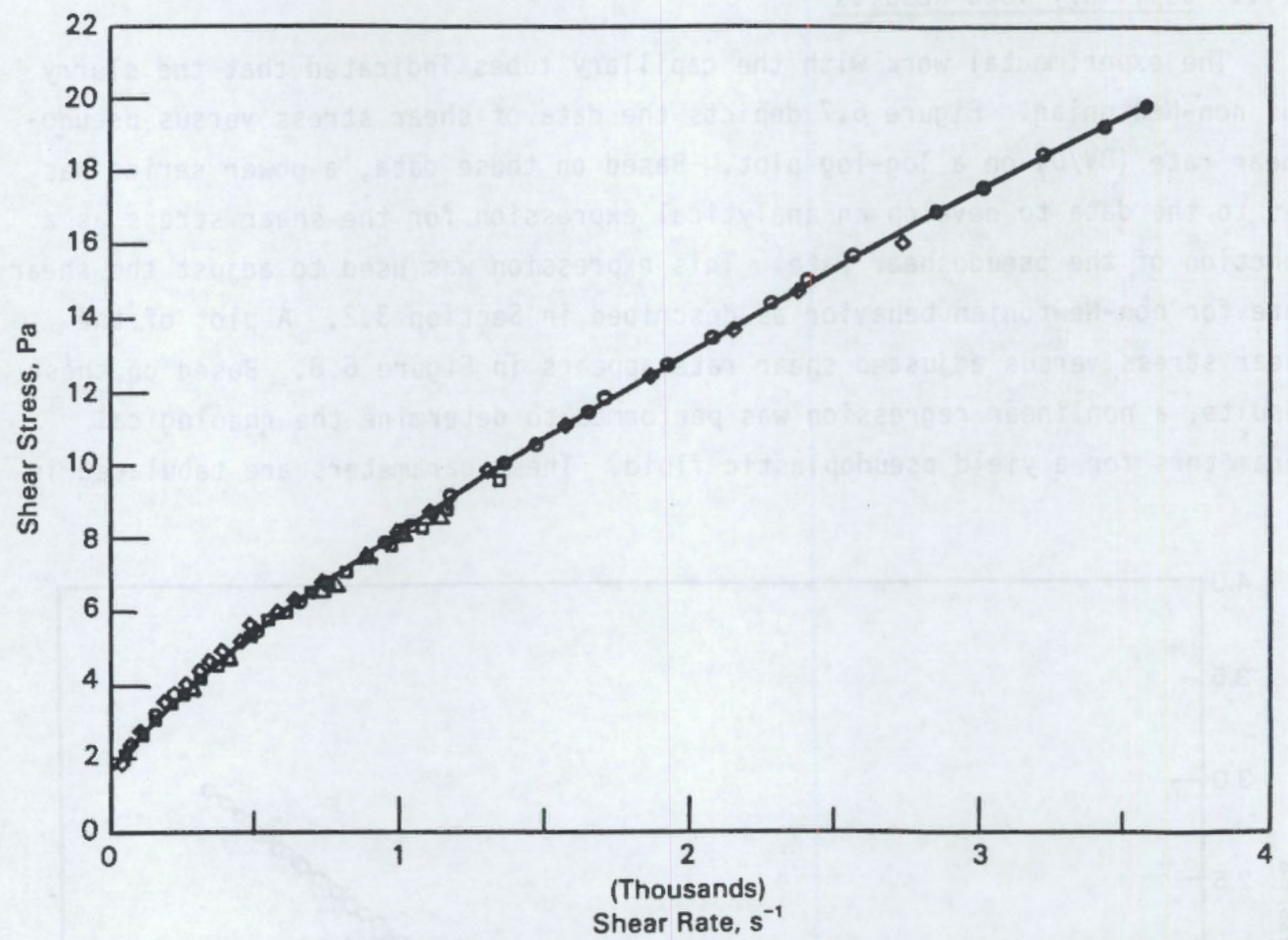

FIGURE 6.8. Shear Stress Versus Shear Rate for WV-205 S7urry Determined Using the Capillary Tube Viscometer

Table 6.2 along with corresponding parameters for a Bingham plastic fluid. The rheological parameters determined using the Haake viscometer with the MVI sensor are included in Table 6.2 .

Figure 6.9 is a plot of $Q /\left(\pi R^{3} \tau_{W}\right)$ versus $\tau_{w}$ for a determination of wall slip effects in the capillary tube viscometer. Note that the data lies along a single curve, indicating the absence of wall slip.

\subsubsection{Pipe Loop Results}

The slurry was mixed in a 250-gal tank and pumped to the pipe test 10op (Figure 6.2). By adjusting the pump speed and allowing some of the slurry to bypass the test section, a wide range of flow rates was achieved. 
TABLE 6.2. Capillary Tube Rheological Parameters

\begin{tabular}{|c|c|c|c|}
\hline Instrument & $\frac{\text { Yield Stress, }}{\mathrm{Pa}^{-}}$ & $\begin{array}{l}\text { Consistency } \\
\text { Index, } \mathrm{Pa} \cdot \mathrm{S} \\
\end{array}$ & $\begin{array}{c}\text { Flow Behavior } \\
\text { Index }\end{array}$ \\
\hline \multicolumn{4}{|l|}{$\begin{array}{l}\text { Calculated using the } \\
\text { yield power law model: }\end{array}$} \\
\hline capillary tubes & 1.65 & 0.0250 & 0.81 \\
\hline Haake, MVI sensor & 1.59 & 0.0012 & 0.88 \\
\hline \multicolumn{4}{|l|}{$\begin{array}{l}\text { Calculated using the } \\
\text { Bingham plastic model: }\end{array}$} \\
\hline capillary tubes & 2.67 & 0.0051 & 1.0 \\
\hline Haake, MVI sensor & 1.65 & 0.0056 & 1.0 \\
\hline
\end{tabular}

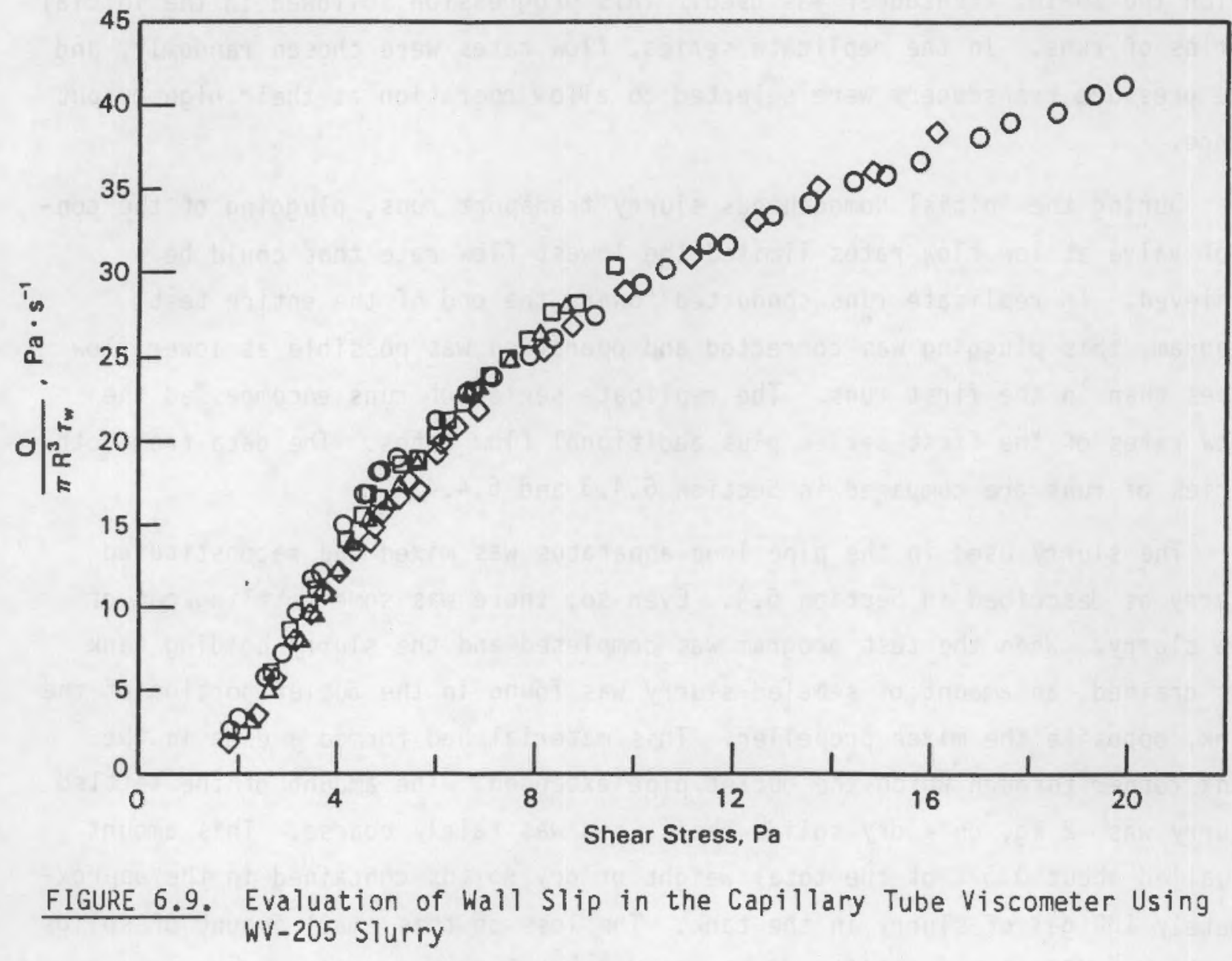


The test program proceeded initially by operating the pump at the speed that gave the highest readings on the 30 psi pressure sensor. The pump speed was decreased and the slurry bypass was adjusted in increments to vary the flow rate. For each flow rate, steady-state pressure drop readings were taken over a 5.5-m-long section of the test loop. As the minimum output signal from an individual transducer was reached, the transducer was changed to one with a lower range. Slurry flow was restarted at the flow corresponding to the maximum output of the transducer. The flow rate was measured by means of a graduated cylinder and stopwatch.

For the transport experiments, the experimental program started with high flow rates using the 30 -psi transducer and progressed to lower flow rates in which the 10-in. transducer was used. This progression followed in the initial series of runs. In the replicate series, flow rates were chosen randomly, and the pressure transducers were selected to allow operation at their high output range.

During the initial homogeneous slurry transport runs, plugging of the control valve at low flow rates limited the lowest flow rate that could be achieved. In replicate runs conducted toward the end of the entire test program, this plugging was corrected and operation was possible at lower flow rates than in the first runs. The replicate series of runs encompassed the flow rates of the first series plus additional flow rates. The data from both series of runs are compared in Section 6.4.3 and 6.4.4.

The slurry used in the pipe loop apparatus was mixed and reconstituted slurry as described in Section 6.4. Even so, there was some settling out of the slurry. When the test program was completed and the slurry holding tank was drained, an amount of settled slurry was found in the outlet portion of the tank, opposite the mixer propeller. This material had formed a dune in the tank corner through which the outlet pipe extended. The amount of the settled slurry was $\sim 2 \mathrm{~kg}$, on a dry solids basis, and was fairly coarse. This amount equalled about $0.87 \%$ of the total weight of dry solids contained in the approximately $130 \mathrm{gal}$ of slurry in the tank. The loss of this small amount of solids should not affect the results of the experimental work. 
The experimental results are discussed in two sections. The first section discusses the laminar flow results, from which various rheological models were evaluated. The second section summarizes the results over the complete range of shear rates, laminar and turbulent, from which a determination of the transition Reynolds number and a determination of a plot of friction factor as a function of Reynolds number was obtained.

\subsubsection{Laminar Flow Results}

In manipulating the data for the laminar flow region, the low pressure readings for each transducer were deleted because of the inaccuracy of the transducers at the low end of their ranges. The low-end data were replaced with high-end data from another transducer.

A logarithmic plot of the shear stress as a function of the unadjusted or pseudoshear rate (shear rate not adjusted for non-Newtonian behavior) is depicted in Figure 6.10. (This figure depicts data for all pipe sizes from the initial series of runs with WV-205 slurry. Data from this run and the replicate series were not combined due to slight differences in the rheological characteristics of the slurry. These differences may be attributed to particle attrition.) Note that the relationship between the $\log$ shear stress and $\log$ shear rate is not linear, indicating non-Newtonian fluid behavior, and that the material is not a power law fluid over this range of shear rates. Also note that the data for all three pipe sizes lie along one curve, which indicates that there are no significant end effects and no apparent wall slip.

The data shown in figure 6.10 were used to develop a power law relationship between the shear stress and the pseudoshear rate. Statistical analysis showed that a quadratic relationship, $\tau_{w}=a+b(d u / d r)+c(d u / d r)^{2}$ where $a, b$, and $c$ are constants, was an adequate relationship to describe the data in Figure 6.10. (A cubic fit was not significantly better.) The quadratic expression was used to determine analytically the slope of the logarithmic plot of the shear stress versus shear rate, or $n^{\prime}$, as discussed in Section 3.2. This value was then used to correct the shear rate and obtain the actual shear rate from which various rheological models were tested. The adjusted shear rate varied from 1.1 to 3.5 times the measured shear rate. The difference 


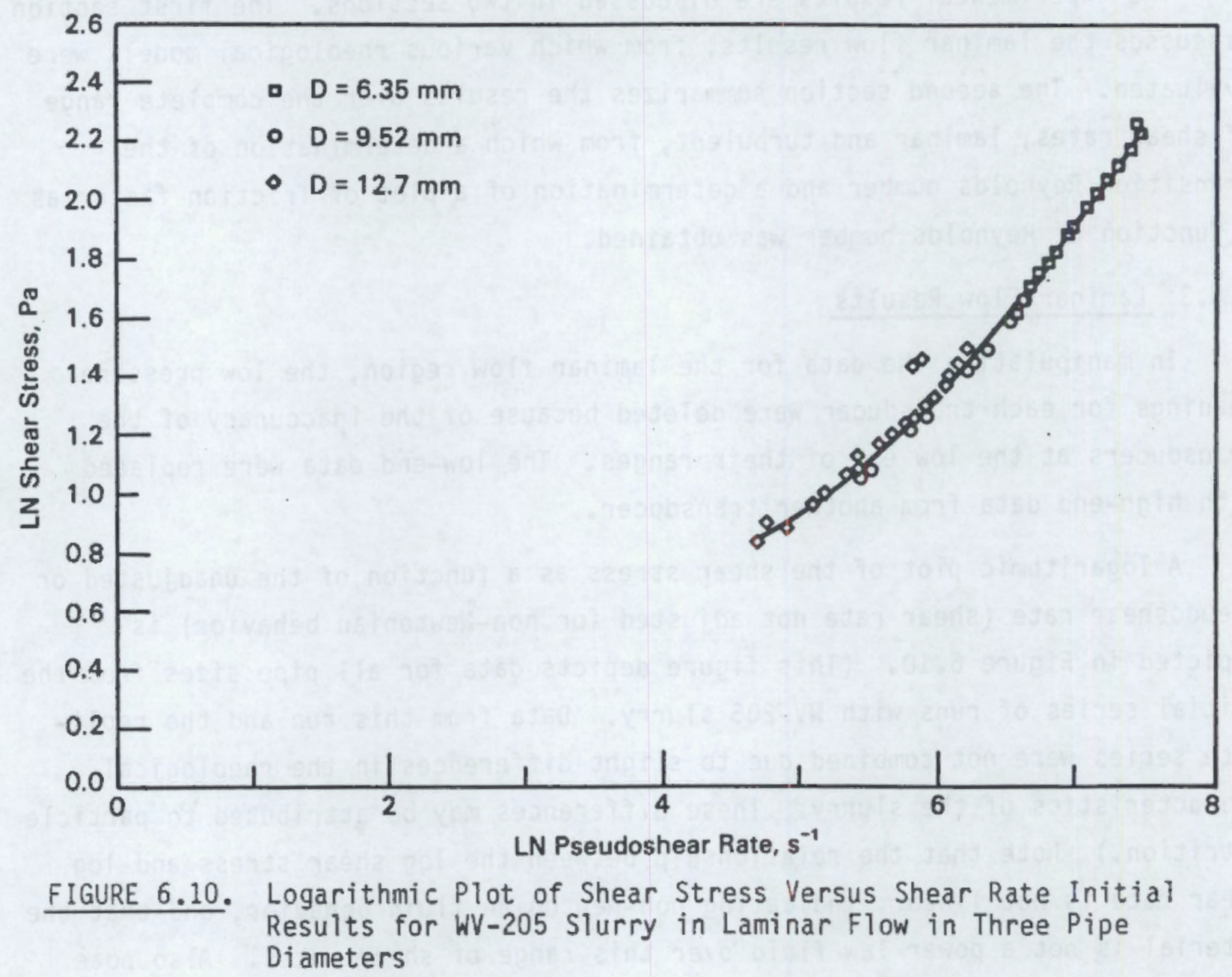

between the two increased with increasing shear rate. The difference verified the need to apply the Mooney-Rabinowitsch method for data analysis.

Table 6.3 summarizes the constants and curve fits for various rheological models. A linear plot of the data is depicted in Figure 6.11 and 6.12 . (The adjusted shear rates are indicated here.) The plots are almost identical and indicate that the fluid can be characterized as either a Bingham plastic (Figure 6.11) or yield pseudoplastic (Figure 6.12).

A logarithmic plot of the shear stress and shear rate for the replicate runs is depicted in Figure 6.13. A quadratic expression was developed and used 
TABLE 6.3. Rheological Constants for Slurry Runs

\begin{tabular}{|c|c|c|c|c|}
\hline \multirow[b]{2}{*}{ Parameter } & \multicolumn{4}{|c|}{ Pipe Size, mm } \\
\hline & 6.35 & 9.52 & 12.7 & $\begin{array}{c}\text { All Pipe Diameters } \\
\text { Combined }\end{array}$ \\
\hline $\begin{array}{l}\text { Yield Pseudoplastic: } \\
\text { Yield stress, Pa } \\
\text { Consistency index, Pa.s } \\
\text { Flow behavior index } \\
r^{2}\end{array}$ & $\begin{array}{l}0.424 \\
0.0253 \\
0.776 \\
0.999\end{array}$ & $\begin{array}{l}0.760 \\
0.0282 \\
0.746 \\
0.999\end{array}$ & $\begin{array}{l}1.409 \\
0.00648 \\
0.954 \\
0.999\end{array}$ & $\begin{array}{l}1.419 \\
0.00801 \\
0.913 \\
0.999\end{array}$ \\
\hline $\begin{array}{l}\text { Bingham Plastic: } \\
\text { Yield stress, } \mathrm{Pa} \\
\text { Consistency index, Pa.s } \\
r^{2}\end{array}$ & $\begin{array}{l}1.922 \\
0.00393 \\
0.994\end{array}$ & $\begin{array}{l}1.552 \\
0.0042 \\
0.997\end{array}$ & $\begin{array}{l}1.491 \\
0.00471 \\
0.993\end{array}$ & $\begin{array}{l}1.704 \\
0.00408 \\
0.999\end{array}$ \\
\hline Hedstrom number & 3078 & 6917 & 12310 & \\
\hline Run temperature, ${ }^{\circ} \mathrm{C}$ & 28 & 28 & 28 & \\
\hline
\end{tabular}

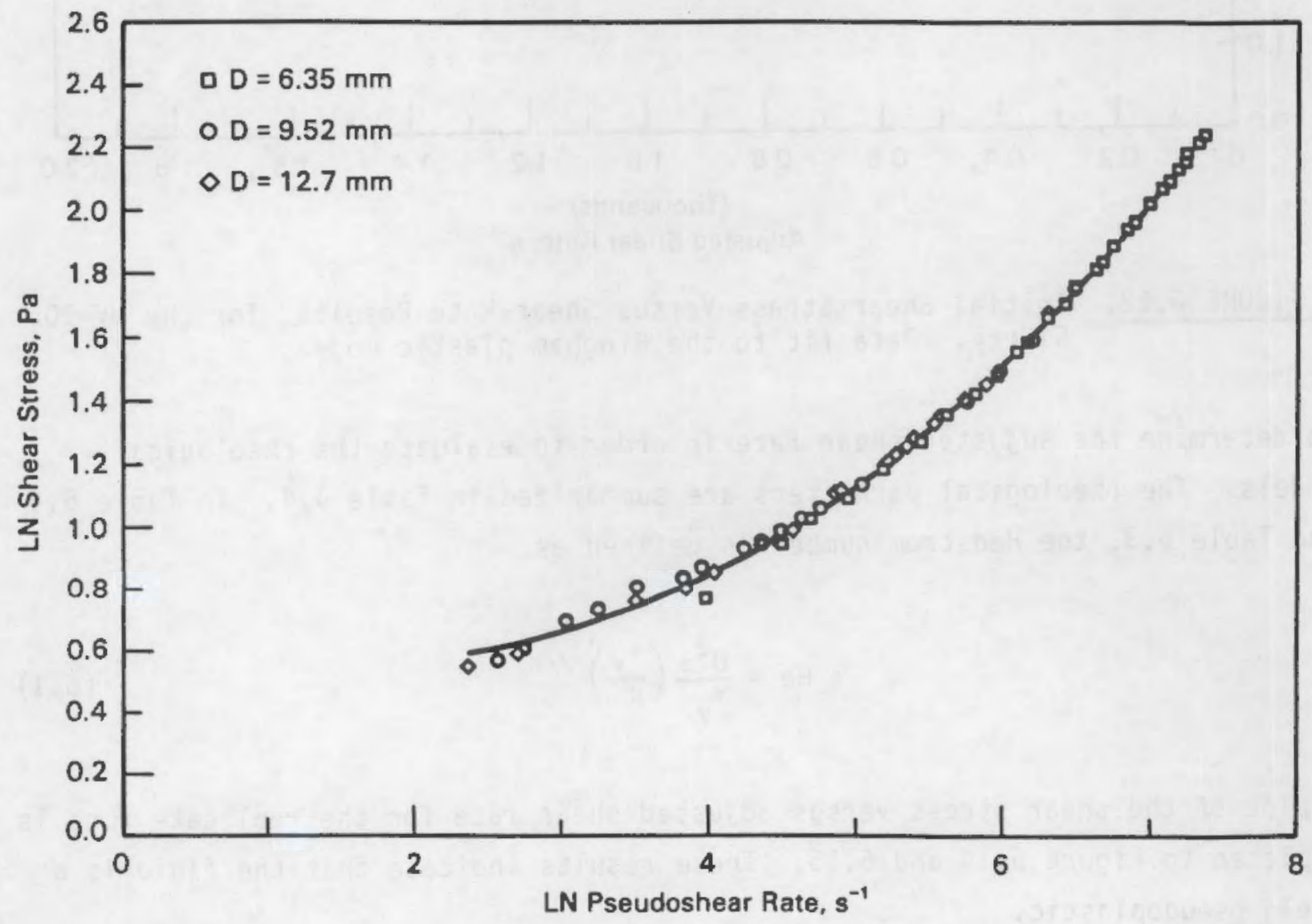

FIGURE 6.11. Initial Shear Stress Versus Shear Rate Results for the WV-205 Slurry. Data are fit to the Bingham plastic model. 


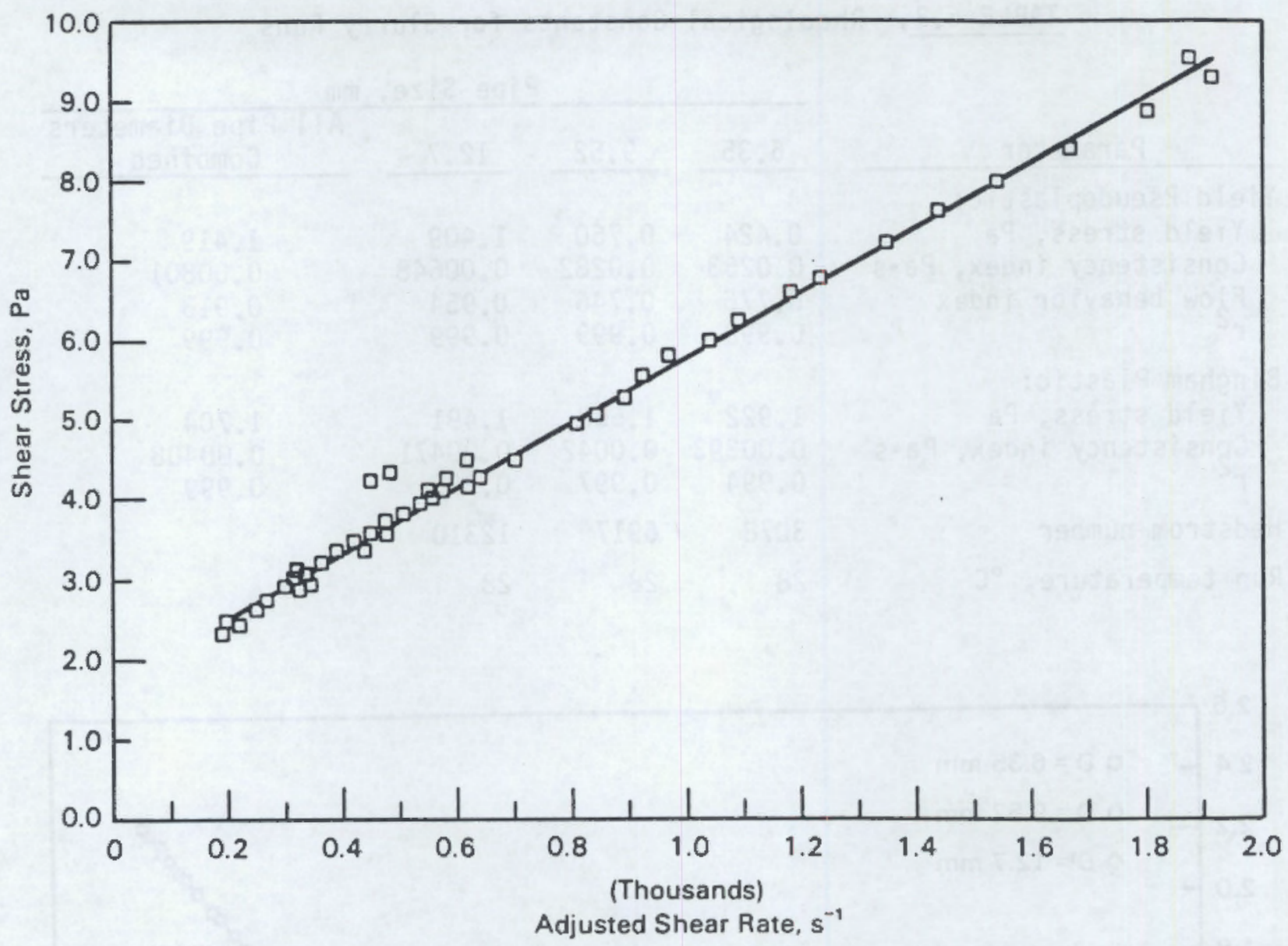

FIGURE 6.12. Initial Shear Stress Versus Shear Rate Results, for the WV-205 Slurry. Data fit to the Bingham plastic model.

to determine the adjusted shear rate in order to evaluate the rheological model5. The rheological parameters are summarized in Table 6.4. In Table 6.4 and Table 6.3, the Hedstrom number is defined as

$$
H e=\frac{D^{2} \rho}{\tau y}\left(\frac{\tau y}{k}\right)^{2 / n}
$$

A plot of the shear stress versus adjusted shear rate for the replicate runs is depicted in Figure 6.14 and 6.15 . These results indicate that the fluid is a yield pseudoplastic. 


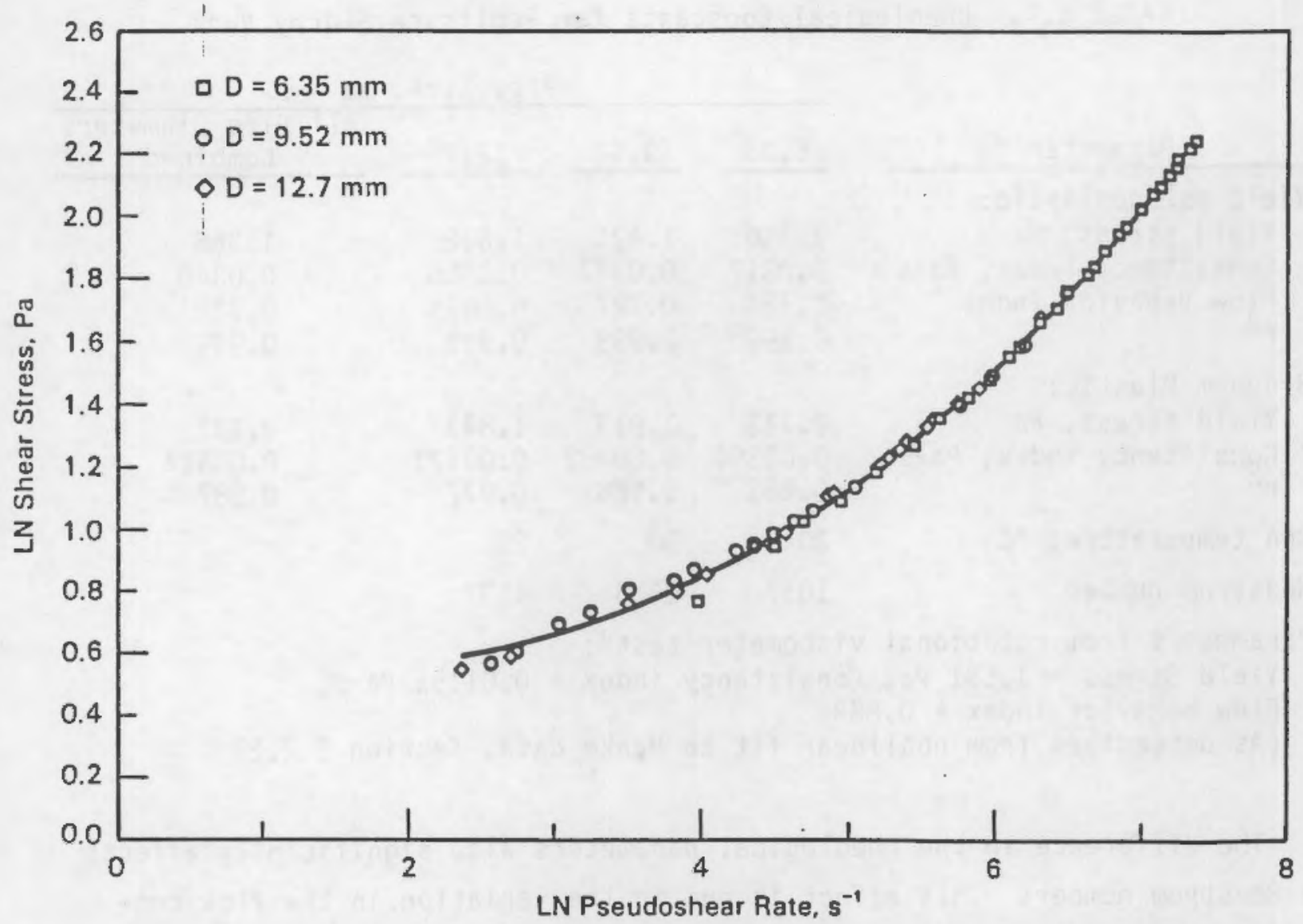

FIGURE 6.13. Logarithmic Plot of Shear Stress Versus Shear Rate (Later Results) for the WV-205 Slurry in Laminar Flow in Three Pipe Diameters

Note that the yield stresses determined for the replicate runs agree fairly well with the initial results, whereas the flow behavior and consistency indices differ. A plot of the two runs is shown as Figure 6.16. Data from initial runs are about $10 \%$ or less below the second series of runs. This difference is probably not due to experimental error because the data for all pipe sizes lie along the two curves. Experimental bias cannot be ruled out, however, because the first series of runs was conducted in steps of decreasing velocities, and the replicate runs were conducted with randomly selected velocities. The slurry nature may have changed somewhat due to particle attrition or settling in the tank or temperature change during the pipe loop experiments. 
TABLE 6.4. Rheological Constants for Replicate Slurry Runs

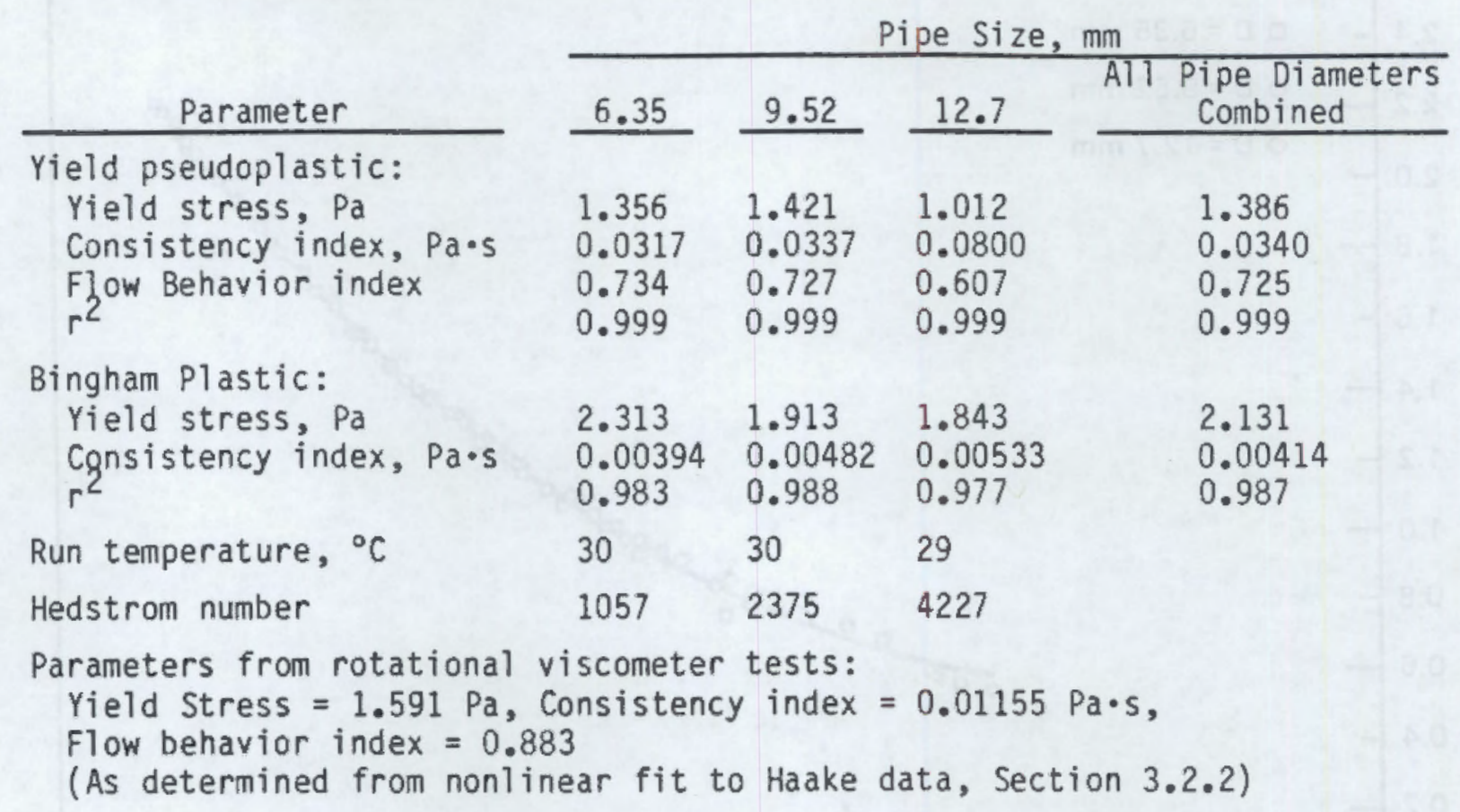

The difference in the rheological parameters also significantly affects the Hedstrom number. This effect is due to the variation in the flow consistency index between the two series of runs.

\subsubsection{Laminar and Turbulent Results}

Figures 6.17 through 6.19 summarize data in the form of logarithmic plots of the shear stress versus shear rate over the complete range of shear rates for each of the pipe diameters for the replicate runs. The replicate series of runs was over a wider range of shear rates.

Figure 6.20 is a composite of all three pipe diameters. Note that in the laminar region the data lie along one line, but in the turbulent region three curves are evident. This difference is due to the different shear rates corresponding to the laminar-turbulent transition point for each pipe. Beyond the laminar-turbulent transition point, the three curves are approximately parallel. Table 6.5 summarizes the shear rates, shear stresses, velocities, and Reynolds numbers corresponding to the transition between laminar and turbulent flow based upon the results shown in Figure 6.20 . 


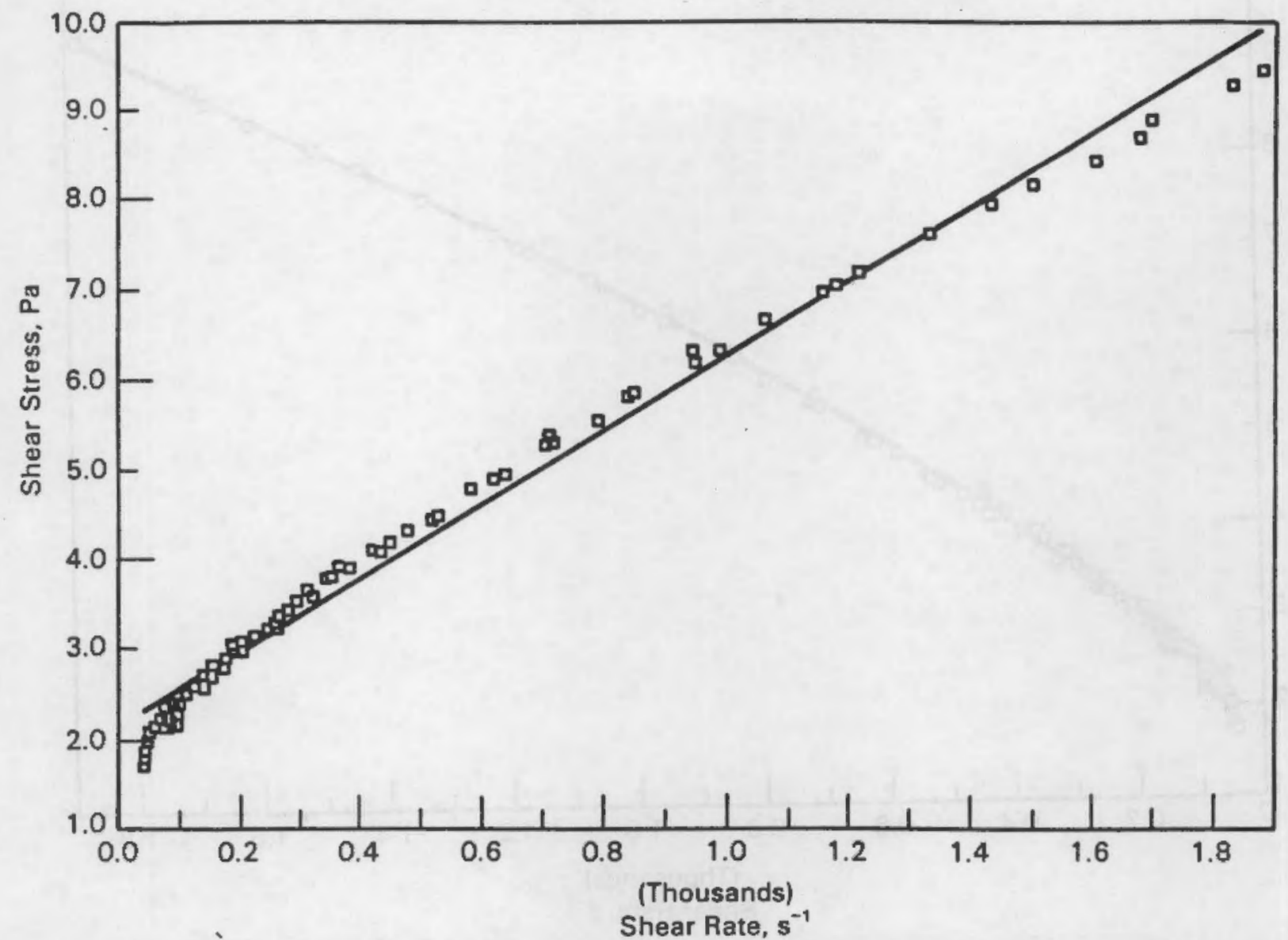

FIGURE 6.14. Shear Stress Versus Shear Rate in Replicate Runs (Later Results) for the WV-205 Slurry Fit to the Bingham Plastic Model

TABLE 6.5. Laminar-Turbulent Transition Data. (Replicate run values are in parentheses following initial run values.)

Parameter

Transition values

Flow rate, $\mathrm{mL} / \mathrm{s}$

Velocity, $\mathrm{m} / \mathrm{s}$

Shear rate, $1 / \mathrm{s}$

Shear stress, $\mathrm{Pa}$

Reynolds number

Reynolds calculated

by yield pseudo-

plastic model

Velocity calculated

using the yield

pseudoplastic model, $\mathrm{m} / \mathrm{s}$

\begin{tabular}{|c|c|c|c|c|c|}
\hline \multicolumn{2}{|c|}{6.35} & \multicolumn{2}{|c|}{9.52} & \multicolumn{2}{|c|}{12.7} \\
\hline $\begin{array}{l}42 \\
1.3 \\
1680 \\
8.9 \\
2560\end{array}$ & $\begin{array}{l}(49) \\
(1.6) \\
(1960) \\
(11) \\
(2850)\end{array}$ & $\begin{array}{l}76 \\
1.1 \\
898 \\
5.9 \\
2910\end{array}$ & $\begin{array}{l}(86) \\
(1.2) \\
(1012) \\
(7.2) \\
(2760)\end{array}$ & $\begin{array}{l}115 \\
0.91 \\
572 \\
4.5 \\
3170\end{array}$ & $\begin{array}{l}(140) \\
(1.1) \\
(639) \\
(5.6) \\
(3050)\end{array}$ \\
\hline 2760 & $(2780)$ & 3190 & (3025) & 3620 & $(3250)$ \\
\hline 1.5 & $(1.4)$ & 1.2 & $(1.3)$ & 1.0 & $(1.2)$ \\
\hline
\end{tabular}




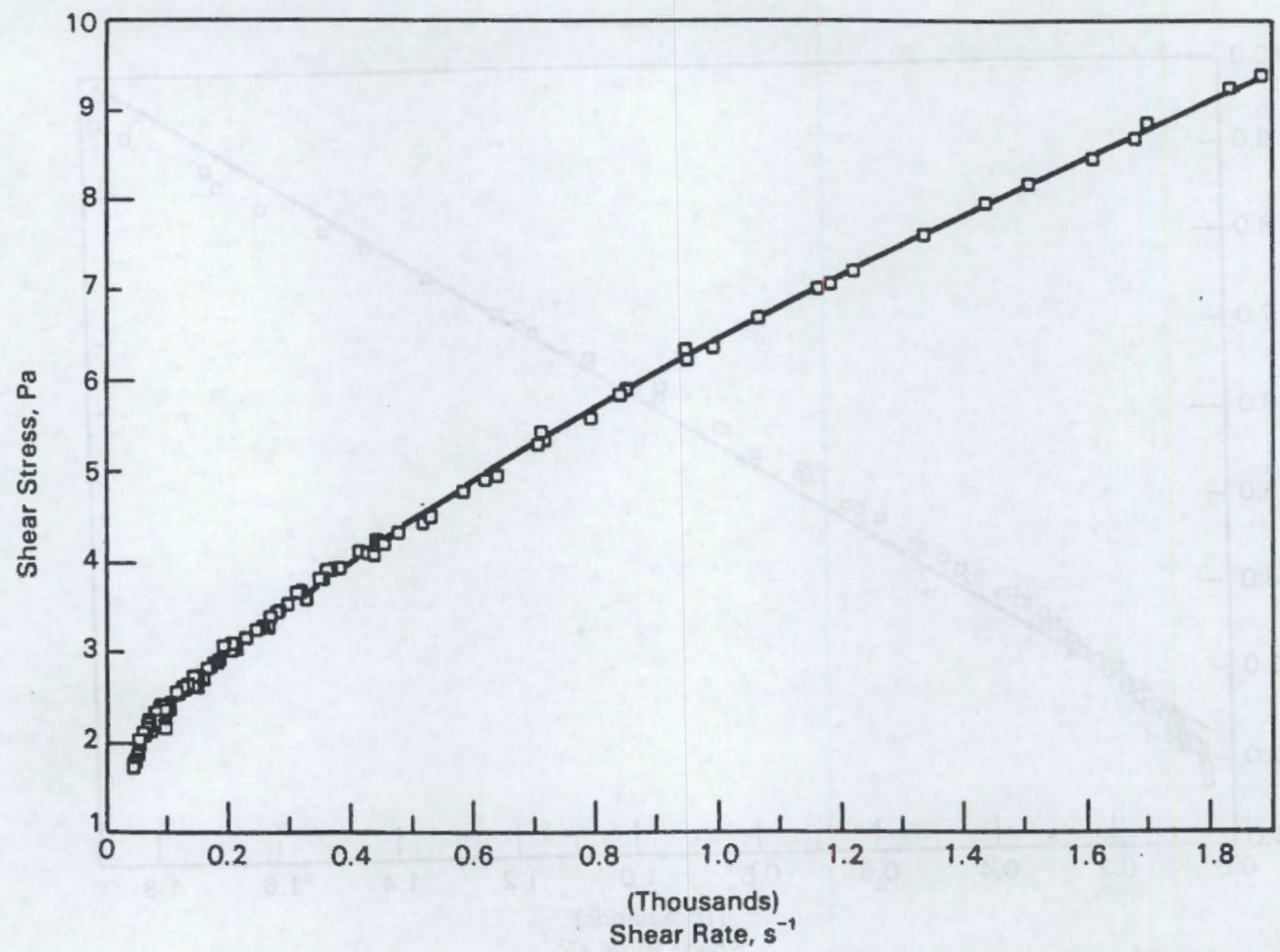

FIGURE 6.15. Shear Stress Versus Shear Rate in Replcate Runs for the WV-205 Slurry Data Fit to the Yield Pseudoplastic Model

The Reynolds number shown in Table 6.5 is defined as

$$
R e=2^{3-n}\left(\frac{n}{1+3 n}\right) \frac{D^{n} V^{2-n} \rho}{k}
$$

The calculated Reynolds numbers are based upon Hanks' program (1978) for yield pseudoplastic fluids. The agreement between the measured values of transition Reynolds numbers and those predicted is generally good (within 10\% for most cases). The variation between runs was $10 \%$ or less. 


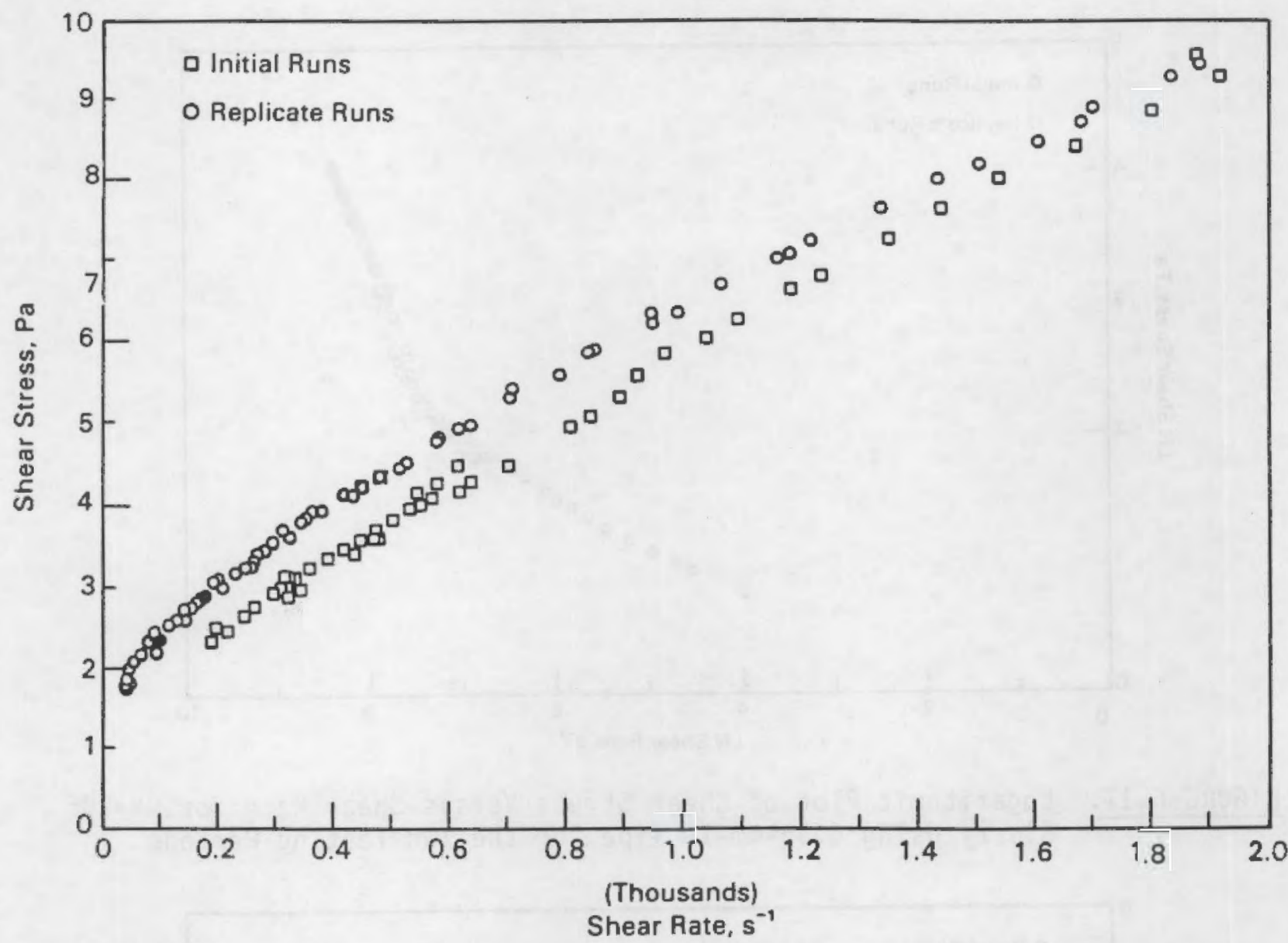

FIGURE 6.16. Shear Stress Versus Shear Rate for WV-205 Slurry for the Two Testing Periods

In Figure 6.20 , the data for the three pipe diameters lie along one curve in the laminar region, indicating that wall slip is probably not significant. If the data were offset between pipe diameters, wall slip would be suspected.

Figure 6.21 is a logarithmic plot of friction factor versus the yield pseudoplastic Reynolds number as defined in Equation 6.2. This plot shows a difference in friction factor as a function of pipe diameter for the laminar region. This difference is within experimental error. This curve could be used to determine pipe pressure drop in the same manner as a Moody diagram or friction factor curve. 


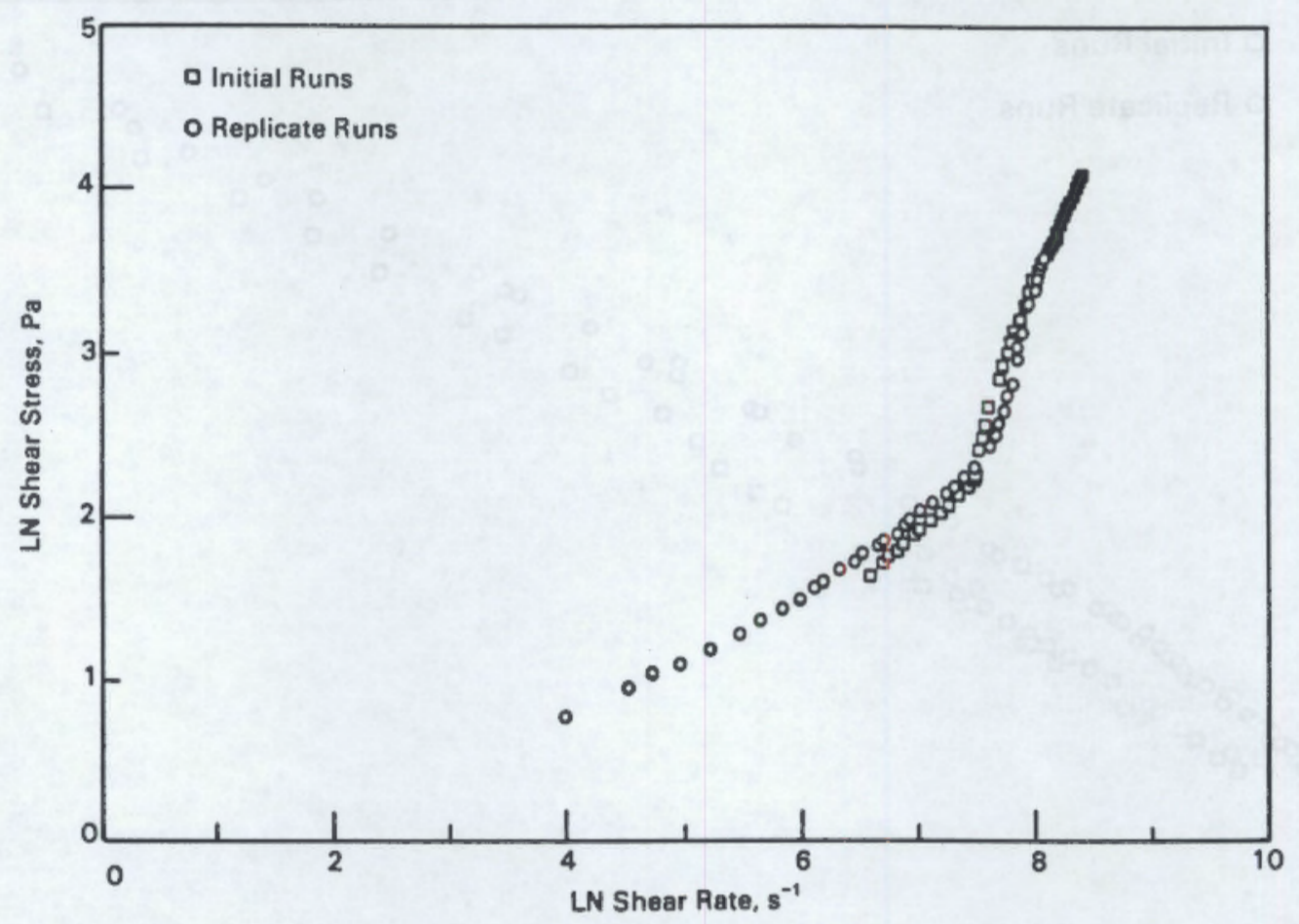

FIGURE 6.17. Logarithmic Plot of Shear Stress Versus Shear Rate for WV-205 Slurry Using 6.35-mm-ID Pipe for the Two Testing Periods

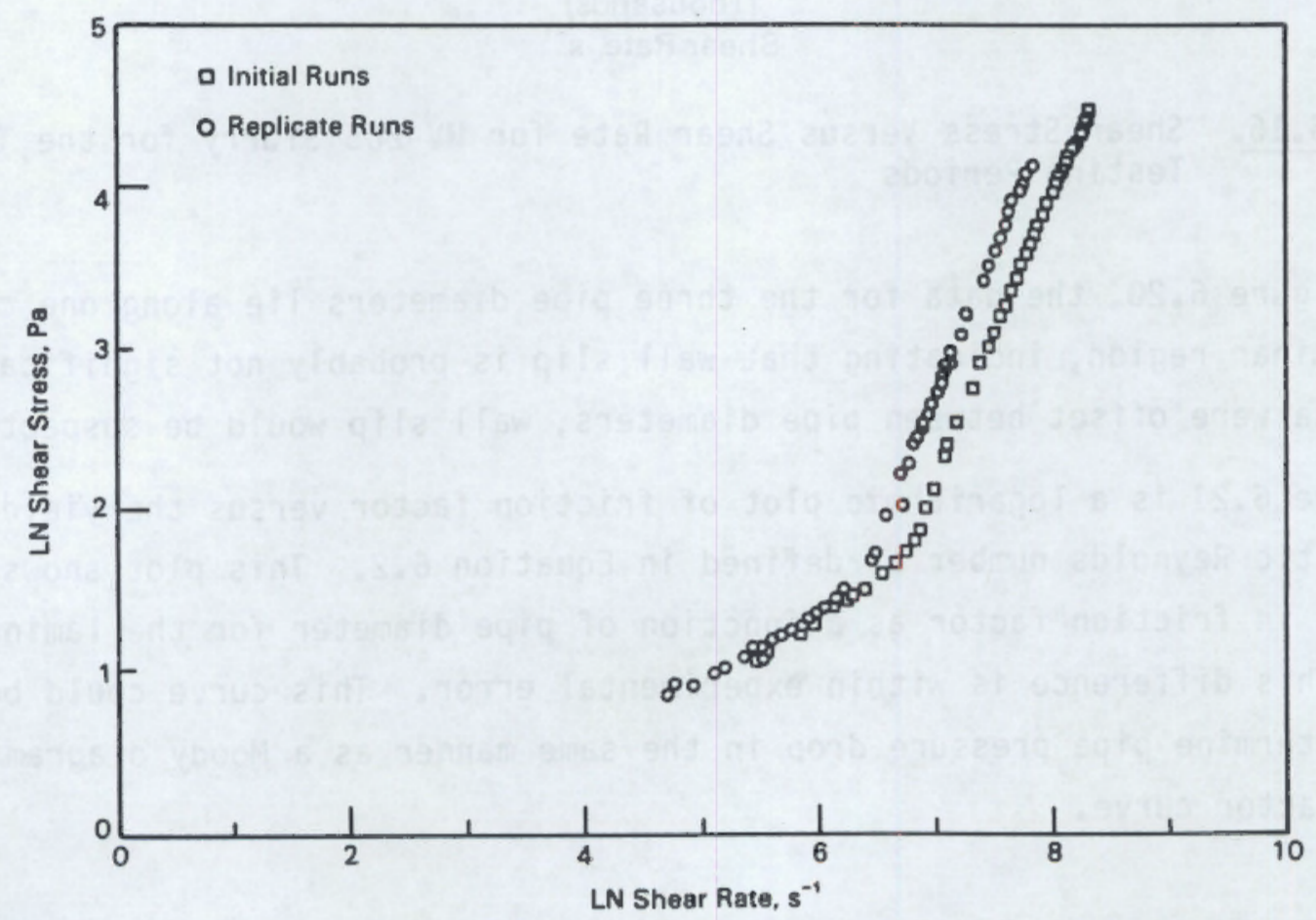

FIGURE 6.18. Logarithmic Plot of Shear Stress Versus Shear Rate for the WV-205 Slurry Using 9.52-mm-ID Pipe for the Two Testing Periods 


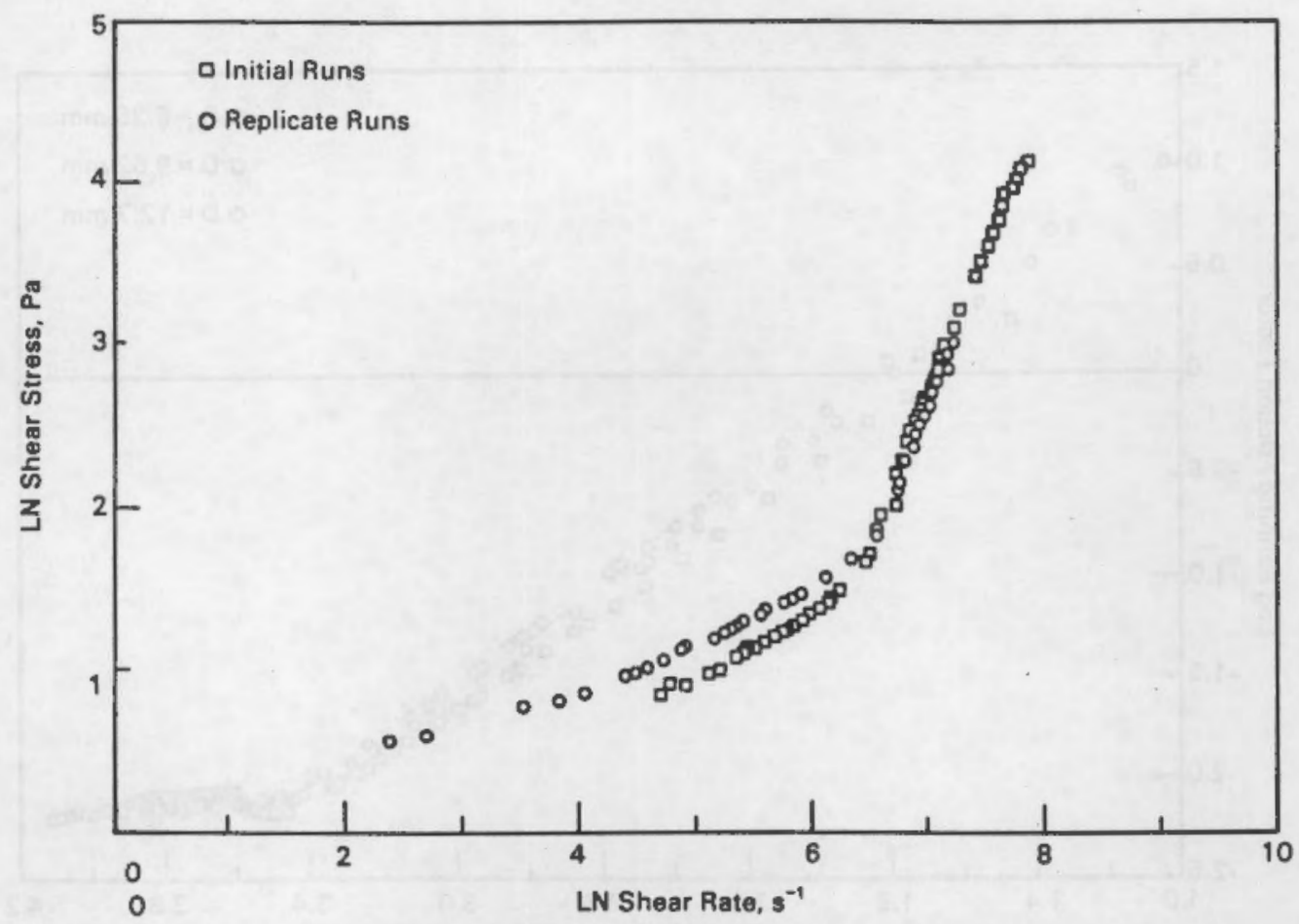

FIGURE 6.19. Logarithmic Plot of Shear Stress Versus Shear Rate for the WV -205 WV-205 Slurry Using 12.7-mm-ID Pipe for the Two Testing Periods

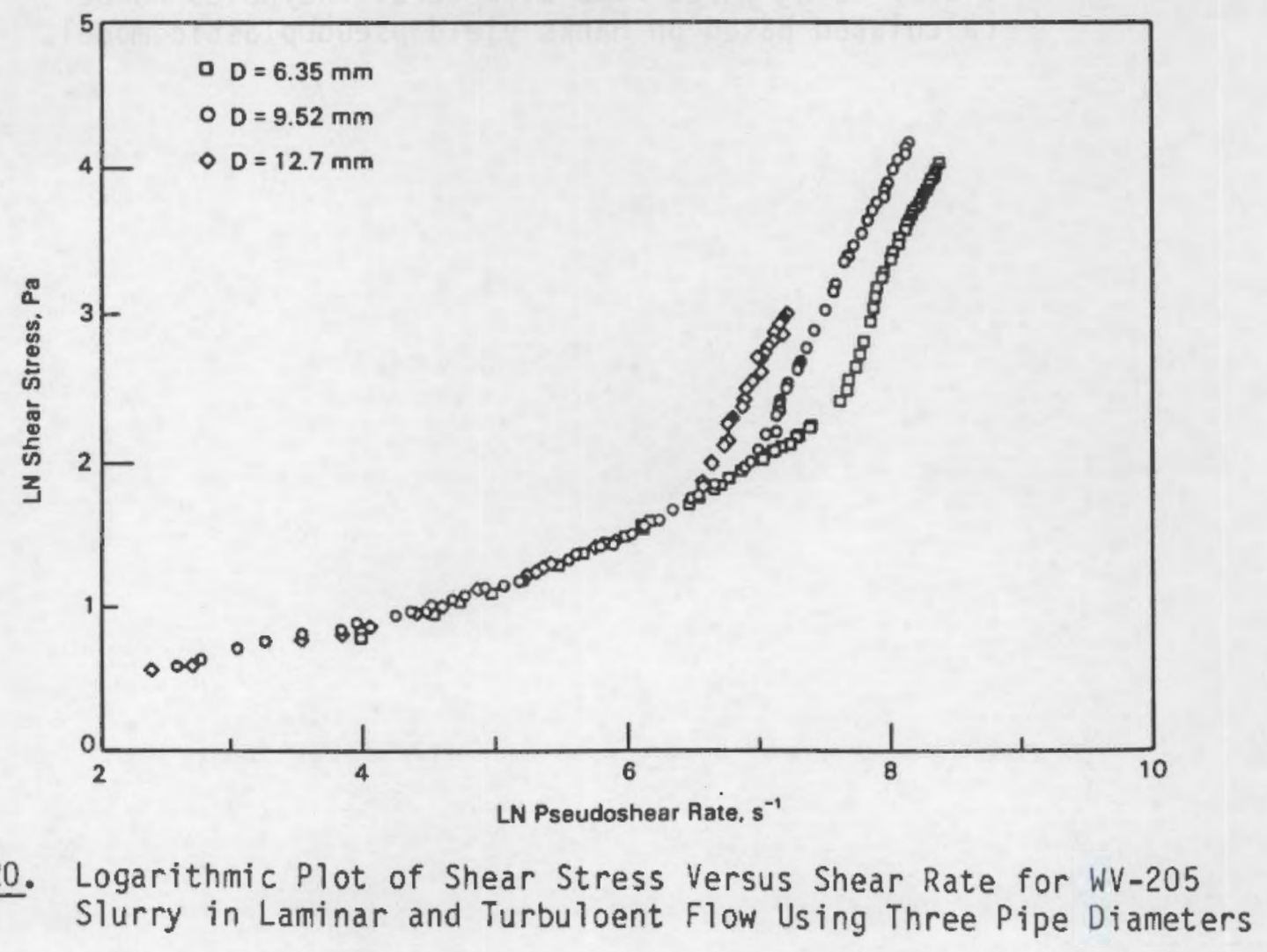




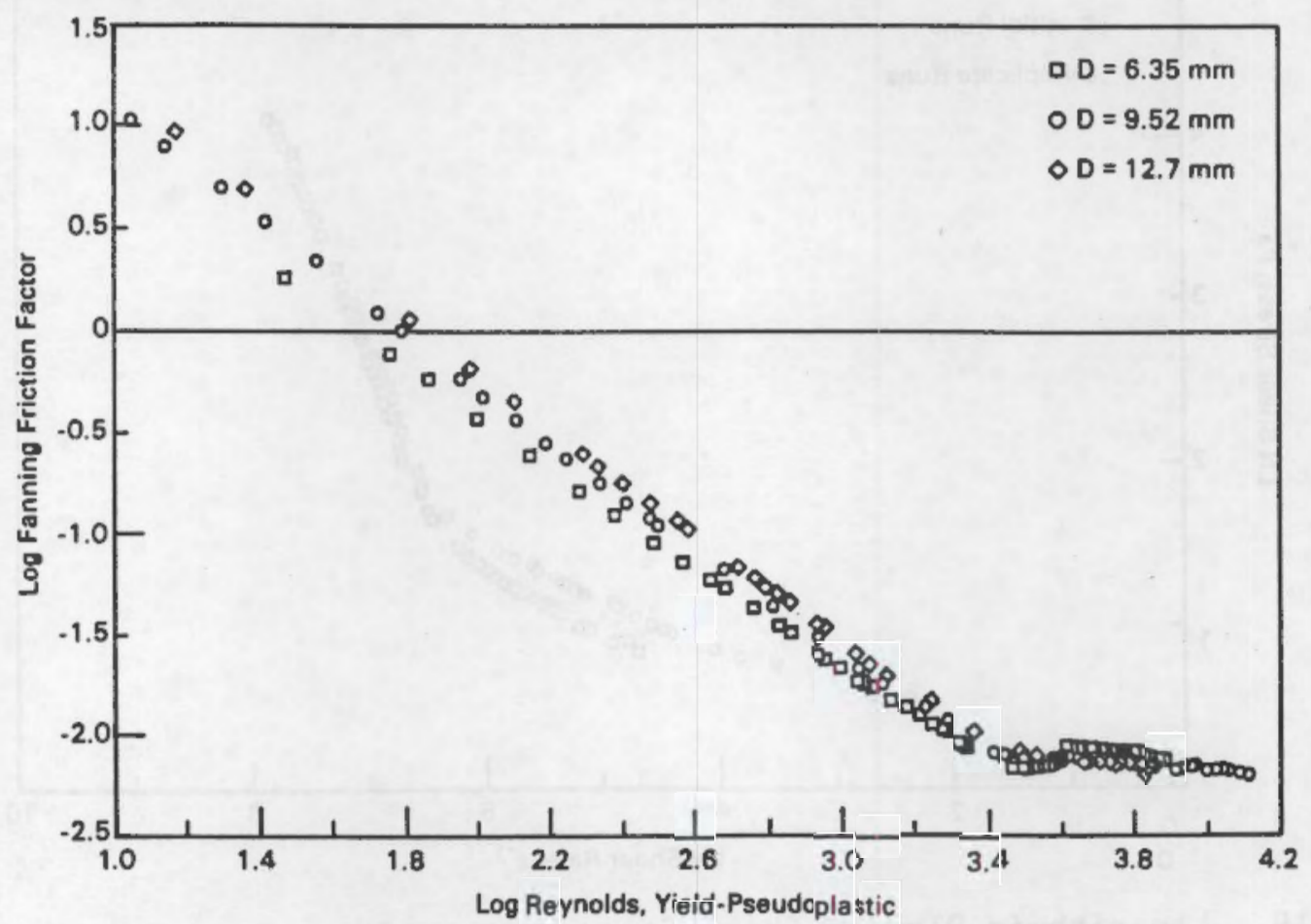

FIGURE 6.21. Fanning Friction Factor Versus Reynolds Number for WV-205 Slurry Using Three Pipe Diameters. Reynolds number calculated based on Hanks yield pseudoplastic model. 


\subsection{RESUSPENSION OF SETTLED SLURRY}

The evaluation of the resuspension characteristics of settled slurry was conducted after the slurry transport experiments. Pipe loop apparatus was used for a series of tests in which the fully suspended slurry was used to resuspend a layer of settled solids. A resuspension apparatus was used for a series of tests in which clear supernate was used to resuspend a layer of settled solids. This experiment allowed visual observation of the resuspension of the solids. Section 7.1 describes the resuspension apparatus. Section 7.2 describes the resuspension experiments and presents the results. Section 7.3 provides an analysis of the resuspension data.

\subsection{DESCRIPTION OF RESUSPENSION APPARATUS}

A small apparatus, shown schematically in Figure 7.1 , was constructed to view the resuspension phenomena for resuspension of the settled slurry by its supernatant. The apparatus was constructed with a $12.7 \mathrm{~mm}$-diameter, $60 \mathrm{~cm}-1$ ong glass tube with ball valves on both ends. The holes in the ball valves were half filled in order to hold the sludge layer while the liquid layer flowed above it. The liquid was supplied from a head tank with a control valve to vary the flow rate. Flow rates were measured using timed volumes. The flow rate at which particle pickup from the sludge layer starts was determined by visual inspection. It was important to determine if the liquid flow was laminar or turbulent when significant particle pickup starts. Particle pickup was visible by particle entrainment in the clear fluid and was checked by calculation of a Reynolds number. Tests were conducted at flow rates incompassing the laminar flow region into the turbulent region.

The pipe flow test loop with clear 24-ft test sections described in Section 6.1 .2 was used to study characteristics of fully suspended slurry being used to resuspend a sludge layer of settled slurry. The study consisted of allowing slurry to settle in the test section for 20 and $44 \mathrm{~h}$. The depth of the sludge layer was measured with a ruler. Fully suspended slurry was then pumped through the test section at a set flow rate. The differential pressure across the test section was measured as a function of time. The sludge layer 


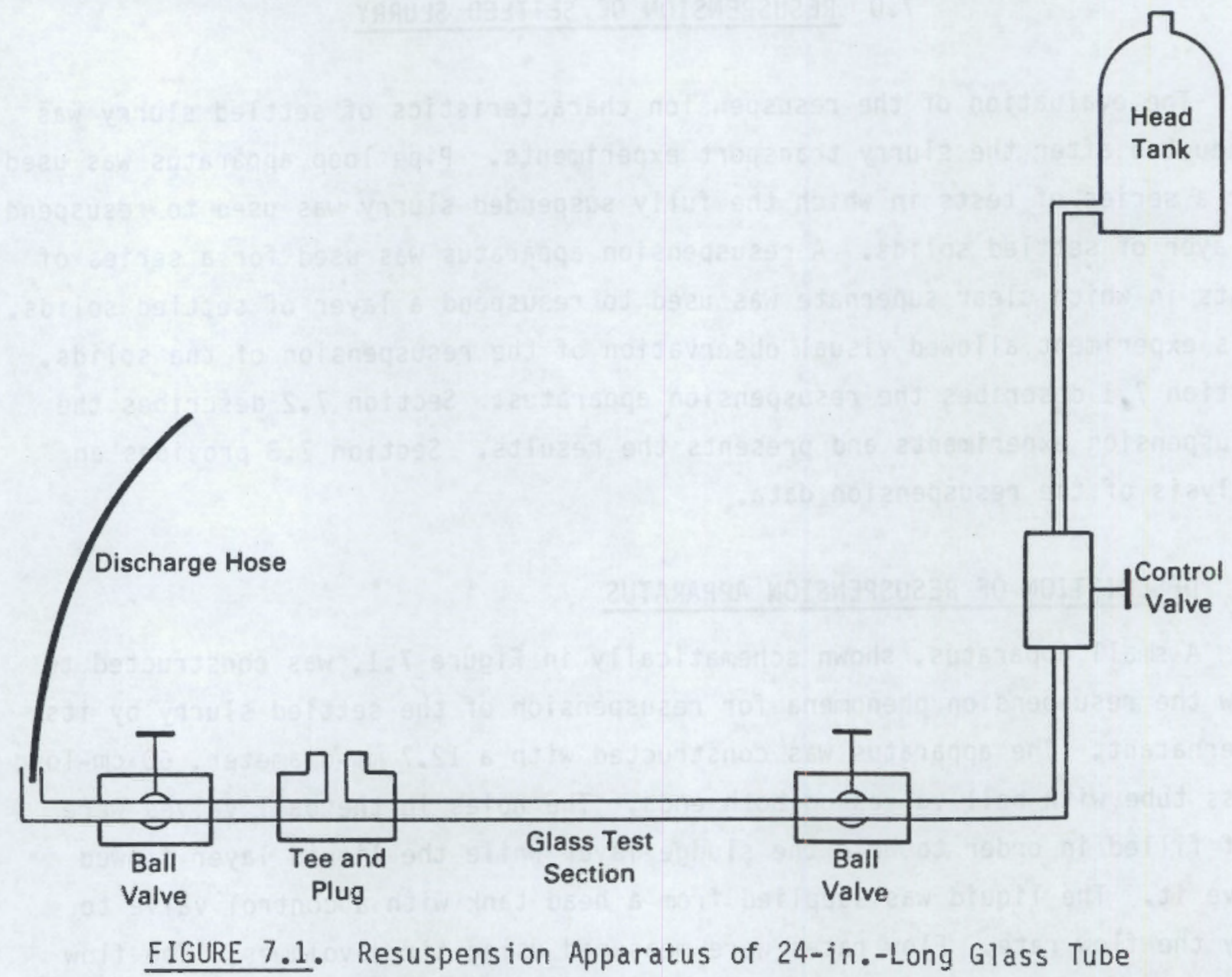

represented a restriction of the normal flow area and hence a greater differential pressure drop for a given flow rate was obtained when the sludge layer was present.

A minimum of six runs at each of the two settling times was made. The flow rates started in the laminar region and were increased into the turbulent region. When the flow rate reached the point at which the differential pressure decreased rapidly with time, the necessary velocity to resuspend the sludge was reached. When the differential pressure decreased to the alreadyknown steady-state conditions, the sludge layer was considered to be fully suspended. Observation of resuspension with water supported this methodology. The initial resuspension phenomena was photographed with a high-speed movie camera to further investigate the active resuspension mechanisms. 


\subsection{RESUSPENSION RUNS}

All of the experiments summarized in this section were performed with settled 36 wt\% WV-205 slurry at the two settling times. The experiment was conducted at room temperature. Resuspension with settled supernatant or water was visually evaluated in the clear glass laboratory scale flow tube described in Section 7.1. Resuspension with fully suspended slurry was evaluated in the pipe loop apparatus. The resuspension of the settled slurry with water was also visually observed in the pipe loop.

\subsubsection{Laboratory Flow Tube Experiments}

The tests were conducted using the small resuspension apparatus. A highspeed movie camera was used to record the information. For increasing flow rates, the following phenomena were observed: 1) at low laminar flow rates, some particle motion on top of the sludge layer was observed, 2) at slightly higher laminar flow rates, traverse and longitudinal dune formation was observed on top of the sludge layer, 3) at higher laminar flow rates, the top of the sludge layer was in motion with small particles being suspended and large particles rolling along the surface, 4) at transition flow rates, there was significant erosion of the sludge layer, and "scooping out" of pockets in the sludge layer leaving a thin coating on the glass wall but little blockage of the pipe, 5) at turbulent flow rates, the settied slurry was rapidly suspended and washed away.

There was significant particle pickup and resuspension at laminar flow rates (as based upon flow rate and pipe radius, i.e., nominal pipe radius without considering area occupied by settled slurry). There were also large particles that may cause some heterogeneity. The near-transition flow conditions had a superficial Reynolds number of $\sim 2100$ (based on the nominal pipe diameter, a supernate viscosity of $1 \mathrm{CP}$, and liquid density of $1 \mathrm{~g} / \mathrm{cm}^{3}$, see Section 5.2.1 for physical properties). The flow corresponding to this Reynolds number exhibited some turbulent eddy formation and was sufficient to remove all of the sludge except for a resistant layer adhering to the tube wall. 
The turbulent flow conditions had a superficial Reynolds number of $\sim 4500$. Turbulent flow suspended the sludge layer in 1 to $2 \mathrm{~s}$ and the coating on the tube wall was removed in a short time. It appears that turbulent conditions are sufficient to completely resuspend the settled slurry. Resuspension of the sludge that had settled for $24 \mathrm{~h}$ required a lower velocity than did resuspension of the sludge that had settled for $48 \mathrm{~h}$. This difference was due to the differences in degrees of compaction.

\subsubsection{Pipe Loop Resuspension Experiments}

Table 7.1 summarizes the test plan for the resuspension runs and includes the transition flow rates and velocities for each pipe diameter. A typical plot of pressure drop versus time for the resuspension runs is depicted in Figure 7.2. The pressure drop rises initially as the valve to the test section is opened. Another rise occurs as the bypass valve is closed and the entire flow passes through the test section.

Figure 7.2 summarizes the transient response for flow through the 12.7-mm-ID pipe. After the test section valve is opened, there is a slight pressure peak which levels out until the bypass line is closed. At this point

TABLE 7.1. Resuspension Test Plan (Tests done at 20 and $44 \mathrm{~h}$ settling time)

\begin{tabular}{|c|c|c|c|c|}
\hline \multirow[b]{2}{*}{ Test No. } & \multirow[b]{2}{*}{ Length, $\mathrm{h}$} & \multicolumn{3}{|c|}{$\begin{array}{l}\text { Target Flow Rates } \\
\text { in mL/s for each } \\
\text { Pipe Diameter, mm }\end{array}$} \\
\hline & & 6.35 & 9.25 & 12.7 \\
\hline 1 & 20 & 14.8 & 26.3 & 38.0 \\
\hline 2 & 20 & 29.5 & 51.9 & 76.0 \\
\hline 3 & 20 & 44.3 & 78.3 & 114 \\
\hline 4 & $20 \& 44$ & 58.9 & 104.6 & 152 \\
\hline 5 & $20 \& 44$ & 73.8 & 130.3 & 190 \\
\hline \multicolumn{5}{|c|}{ Transition Flow(a) } \\
\hline Rate, $n$ & & 42 & 76 & 115 \\
\hline Velocit & $\mathrm{m} / \mathrm{s}$ & 1.3 & 1.1 & 0.9 \\
\hline
\end{tabular}

(a) Based on initial series of homogeneous slurry runs 


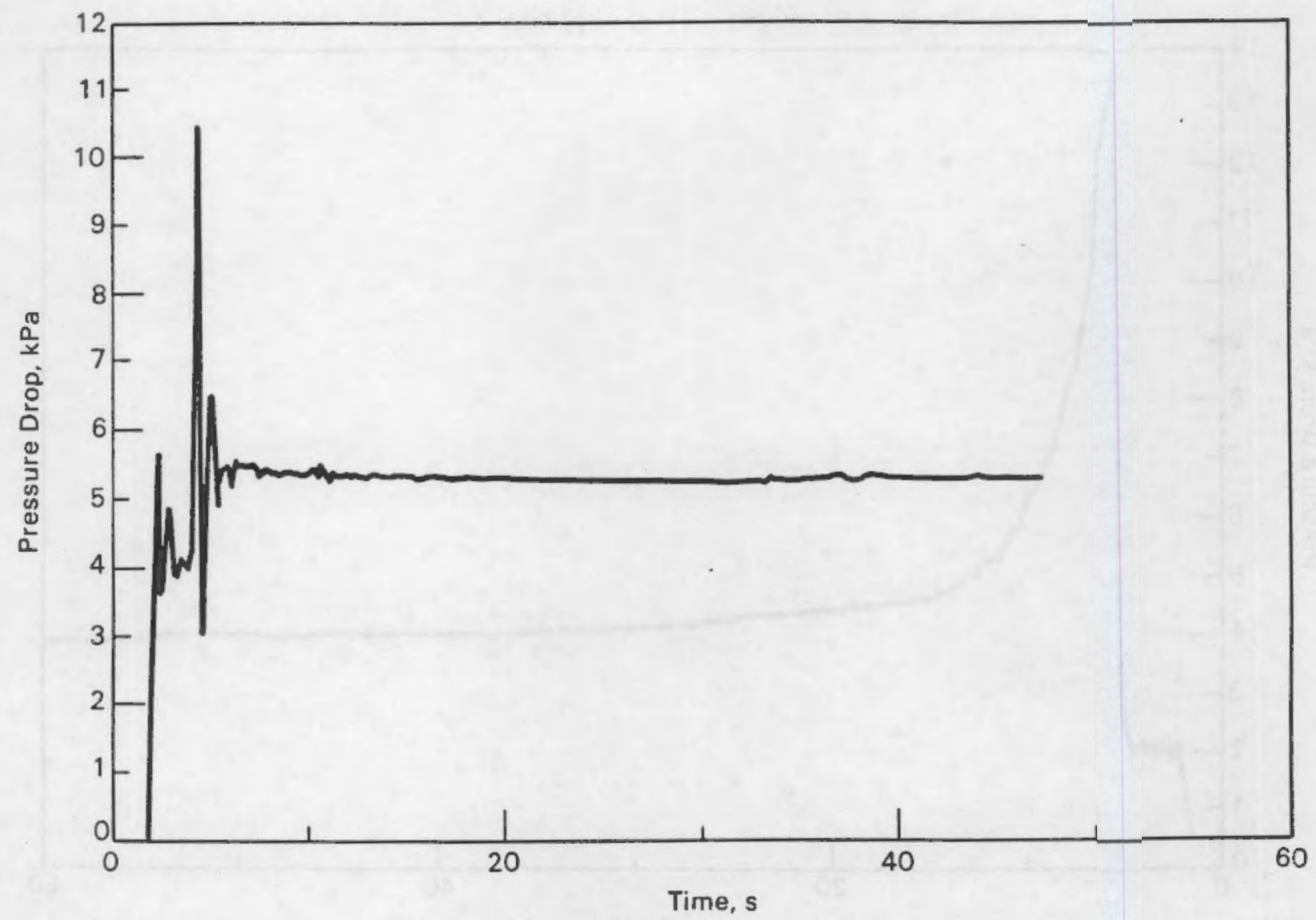

\section{FIGURE 7.2. Pressure Drop Versus Resuspension Time for WV-205 Slurry at a $15.7 \mathrm{~mL} / \mathrm{s}$ Flow Rate After $20 \mathrm{~h}$ of Settling in 12.7-mm-ID Pipe}

there is another pressure peak which dampens quickly as the flow and, consequently, the pressure drop attain steady state. Both pressure peaks are of narrow bandwidth, approximately 1 to $2 \mathrm{~s}$. The transient behavior is essentially a second-order dampened sine wave. The pressure peak amplitude is apparently a function of the flow rate and increases slightly with flow rate. The peak height is between two and three times the final steady-state pressure drop.

Figure 7.2 can be compared to figures 7.3 through 7.7 , for a range of flow rates through the $12.7-\mathrm{mm}-\mathrm{ID}$ pipe with settled sludge. The slurry was allowed to settle over a $20-h$ period and resulted in a bed height of approximately $80 \%$ of the pipe diameter. At the lower flow rates, the transient behavior is 


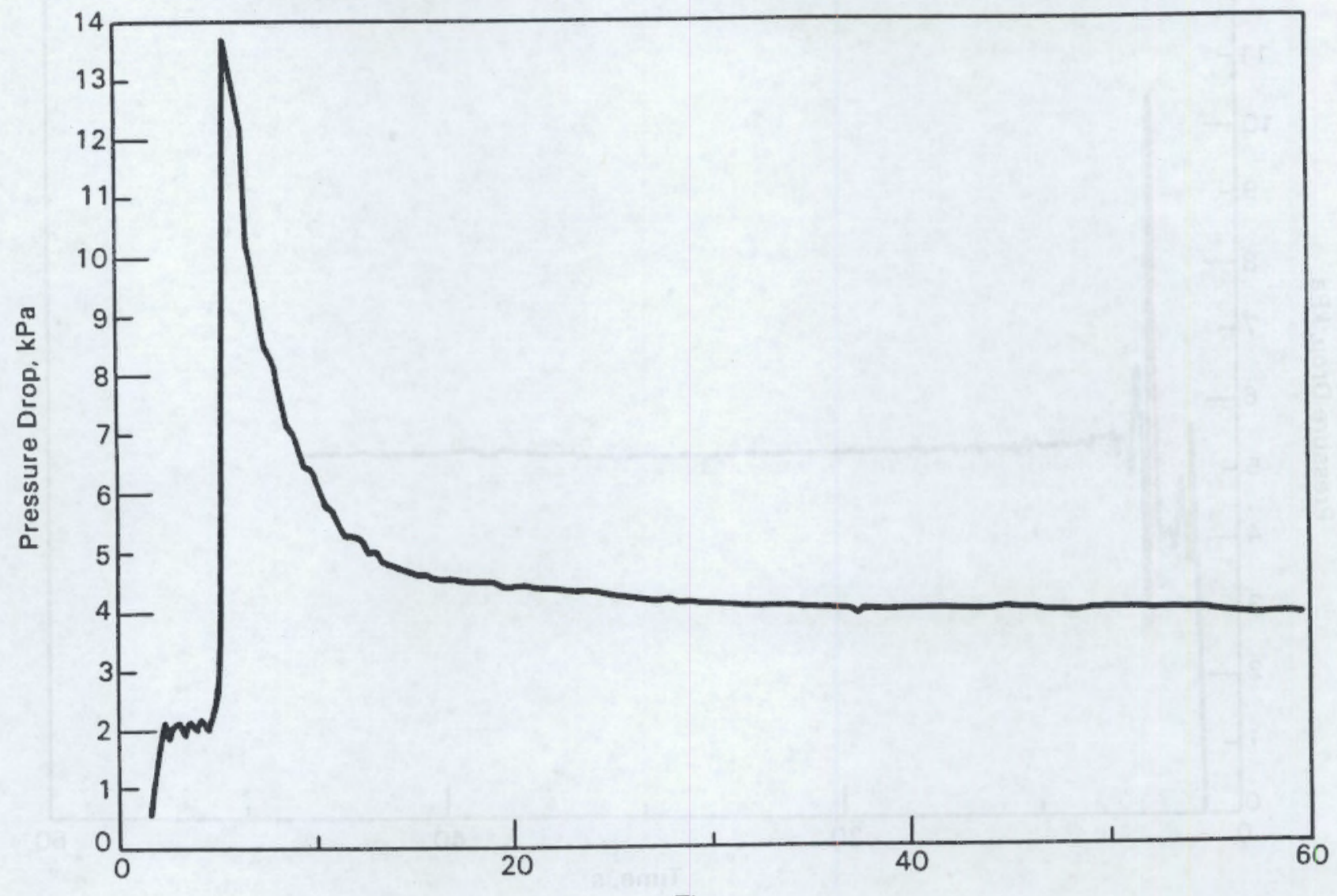

Time, $s$

FIGURE 7.3. Pressure Drop Versus Time for Fully Homogeneous Slurry Transported Through 12.7-mm-ID Pipe

essentially a first-order decay. As the flow rate increases, a second-order response is noted in Figure $7.6(114 \mathrm{~mL} / \mathrm{s})$ which corresponds to a transition Reynolds number flow. The amplitude of the peak increased roughly with flow rate; its bandwidth decreased roughly with flow rate. The time to reach steady-state after the pressure peak varied from approximately $10 \mathrm{~s}$ at a flow rate of $15.7 \mathrm{~mL} / \mathrm{s}$ to approximately $5 \mathrm{~s}$ at $114 \mathrm{~mL} / \mathrm{s}$ and higher. The steadystate curves for flow rates of $114 \mathrm{~mL} / \mathrm{s}$ and below are fairly flat. For higher flow rates, the curves are quite irregular and are probably a consequence of turbulance. At the highest flow rate (Figure 7.7 ), motion pictures were taken of the resuspension. These pictures revealed eddy formation and unsteady-state flow behavior characteristic of turbulent flow. The steady-state pressure drop for fully suspended flow (Figure 7.2) may be compared to that for resuspension 


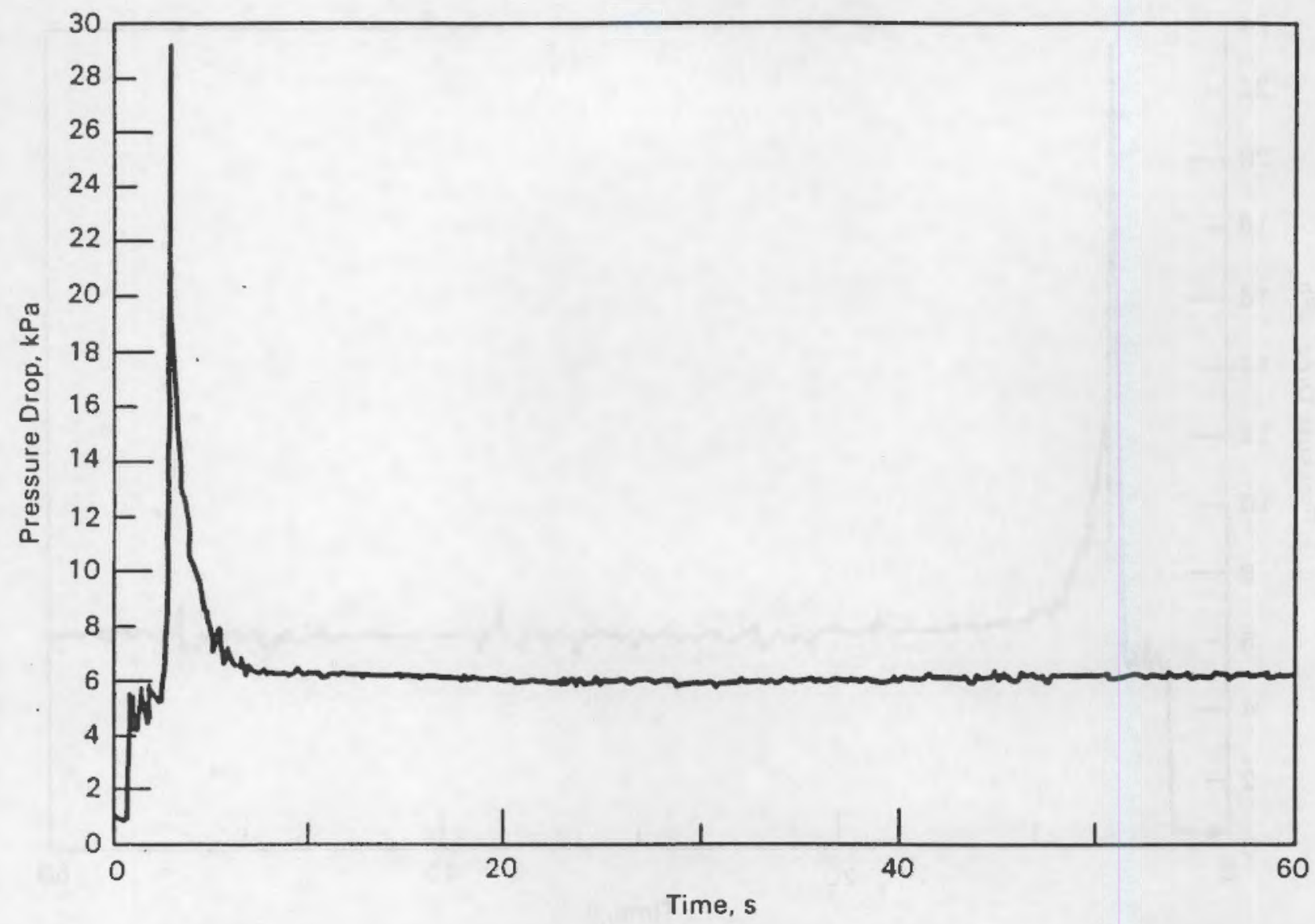

FIGURE 7.4. Pressure Drop Versus Resuspension Time for WV-205 Slurry at a $26.4 \mathrm{~mL} / \mathrm{s}$ flow Rate After $20 \mathrm{~h}$ of Settling in 12.7-mm-ID Pipe

(Figure 7.4). Approximately the same pressure drop is noted, which indicates that the solids have been fully suspended for flows down to approximately $30 \mathrm{~mL} / \mathrm{s}$.

Figures 7.8 and 7.9 show the results for a settling time of $44 \mathrm{~h}$ in the 12.7-mm-ID pipe. Figure 7.8 , at an extremely low flow rate of $0.29 \mathrm{~mL} / \mathrm{s}$, differs from all of the previous figures in that after a pressure peak, the pressure drop continues to rise indefinitely in a somewhat linear fashion. At this low flow rate, the rise in pressure drop may be due to the higherviscosity slurry gradually replacing the lower-viscosity supernatant in the pipe, resulting in the gradual rise in pressure drop. Figure 7.9 (flow rate of $40 \mathrm{~mL} / \mathrm{s}$ ) can be compared to Figure 7.5 with a flow rate of $39.5 \mathrm{~mL} / \mathrm{s}$ after $20 \mathrm{~h}$ settling time. The width of the peak is greater in Figure 7.9 than in 


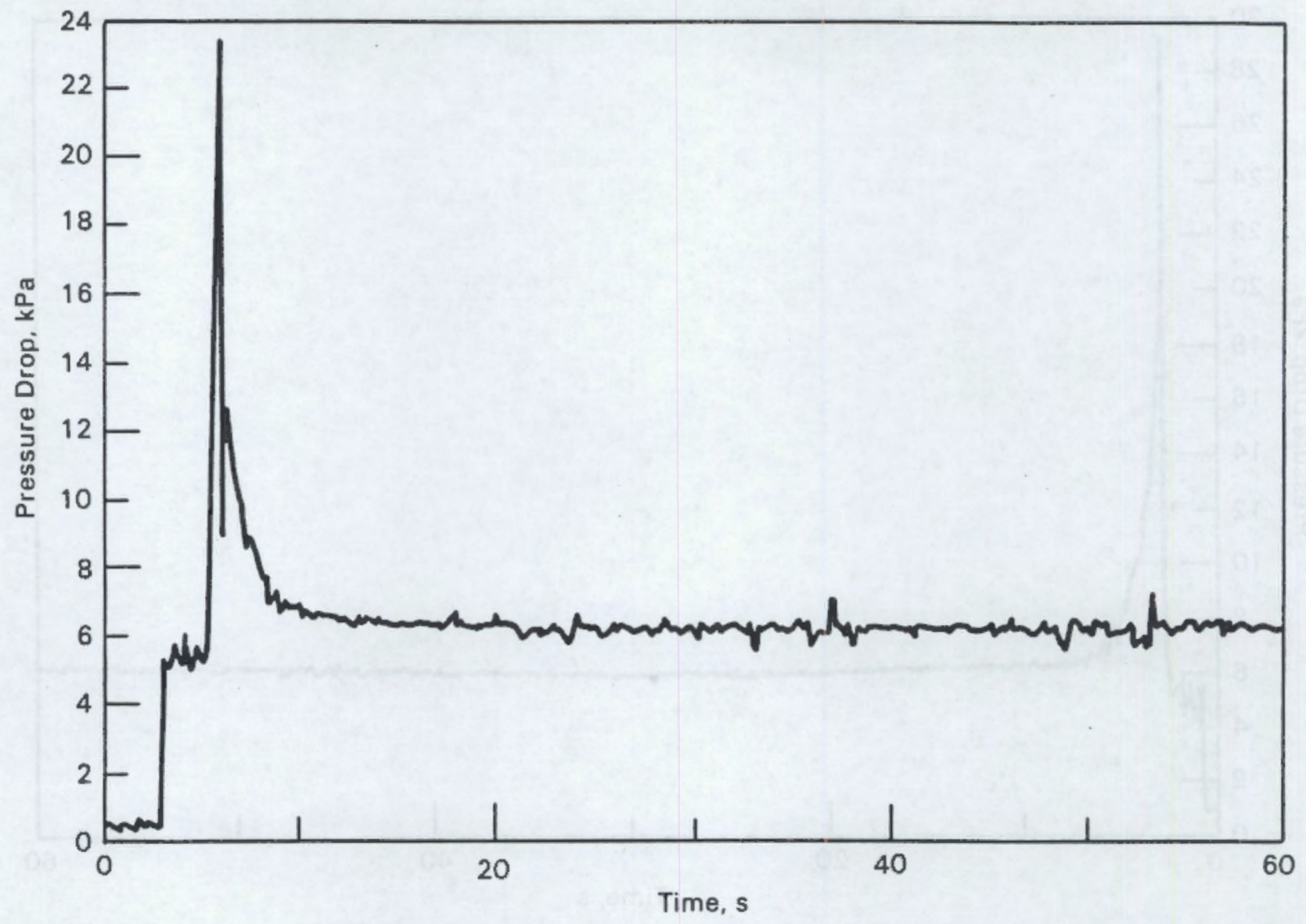

FIGURE 7.5. Pressure Drop Versus Resuspension Time for WV-205 Slurry at a $39.5 \mathrm{~mL} / \mathrm{s}$ Flow Rate After $20 \mathrm{~h}$ of Settling in 12.7-mm-ID Pipe

Figure 7.5 , indicating that the settled sludge may be a little more compacted and take longer to resuspend. The bed height measurements indicate that the sludge has compacted more, i.e., $1.0 \mathrm{~cm}$ for the $44 \mathrm{~h}$ settling versus $1.1 \mathrm{~cm}$ for the $20 \mathrm{~h}$ settling in the $12.7-\mathrm{mm}-10$ pipe.

Somewhat different behavior is noted in the results for the lowest flow rate in the 9.52-mm-ID pipe. Figures 7.10 through 7.14 show similar behavior to the 12.7-mm-ID runs regarding the effect of flow rate upon peak height, bandwidth, and turbulence. Figure 7.11, at the lowest flow rate, however, shows some undulations in the pressure drop that may be due to dune formation in the pipe and the gradual scouring away of these dunes. High-speed motion pictures at the highest flow rate, shown in figure 7.13 , also indicate turbulence. 


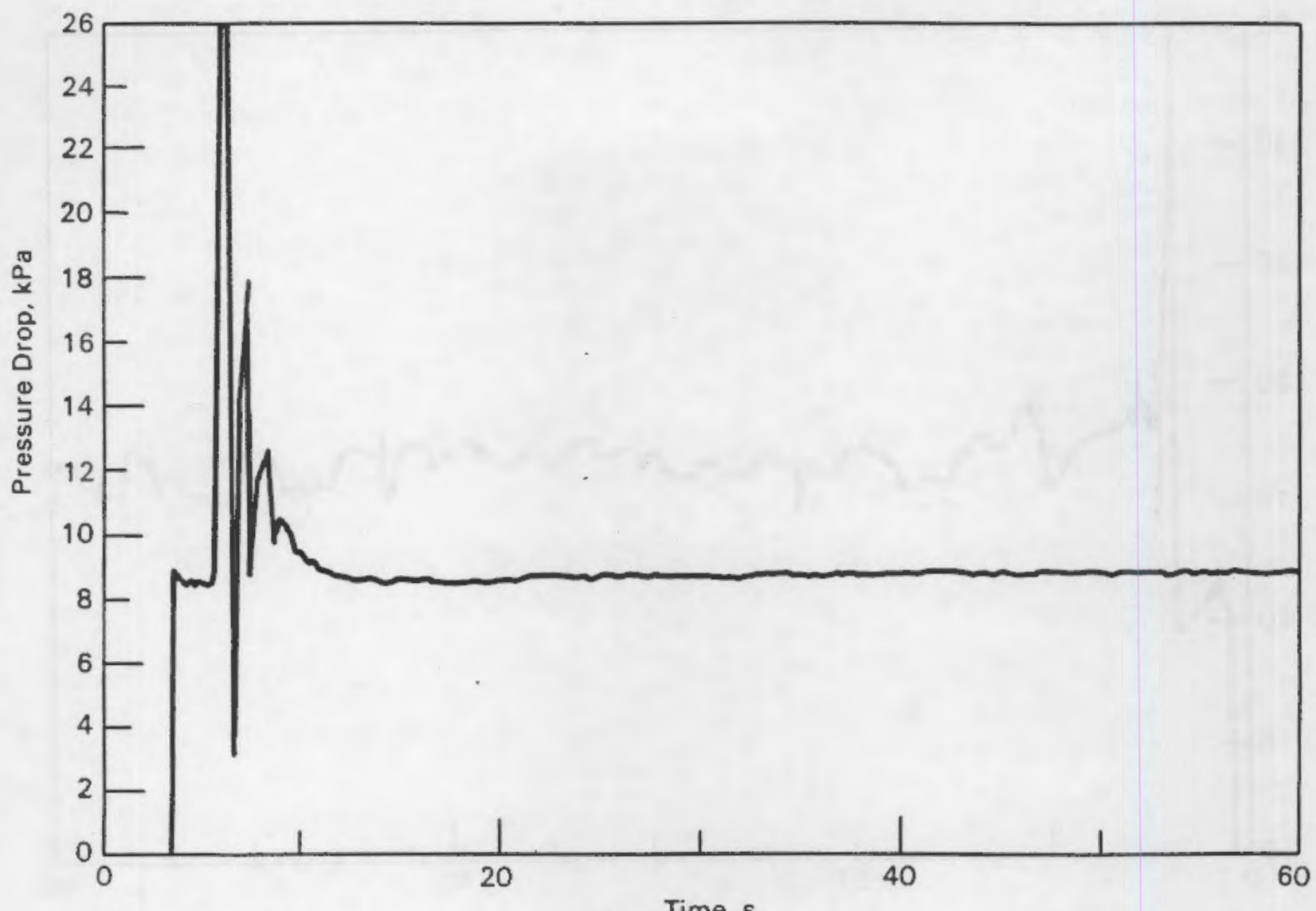

Time, $s$

FIGURE 7.6. Pressure Drop Versus Resuspension Time for WV-205 Slurry at a $114 \mathrm{~mL} / \mathrm{s}$ Flow Rate After $20 \mathrm{~h}$ of Settling in 12.7-mm-ID Pipe

Figure 7.14 for the 44-h resuspension test when compared to a similar plot (Figure 7.11) for a higher flow rate shows trends similar to those observed for the 12.7-mm-ID pipe runs. The same steady-state pressure is attained, although the bandwidth for the 44-h pressure peaks is somewhat greater than those for the $20 \mathrm{~h}$ settling times which indicates slightly higher compaction of the sludge at the longer settling times but no other differences in resuspension.

Figures 7.15 through 7.18 depict the results for the 6.35 -mm-ID pipe tests. Similar trends concerning peak pressure drop, bandwidth and turbulance are seen relative to the plots for the other pipe sizes. After the onset of turbulance, however, the initial pressure peak is lower than the final steadystate pressure (see Figures 7.17 and 7.18 ). Perhaps the initial pressure drop 


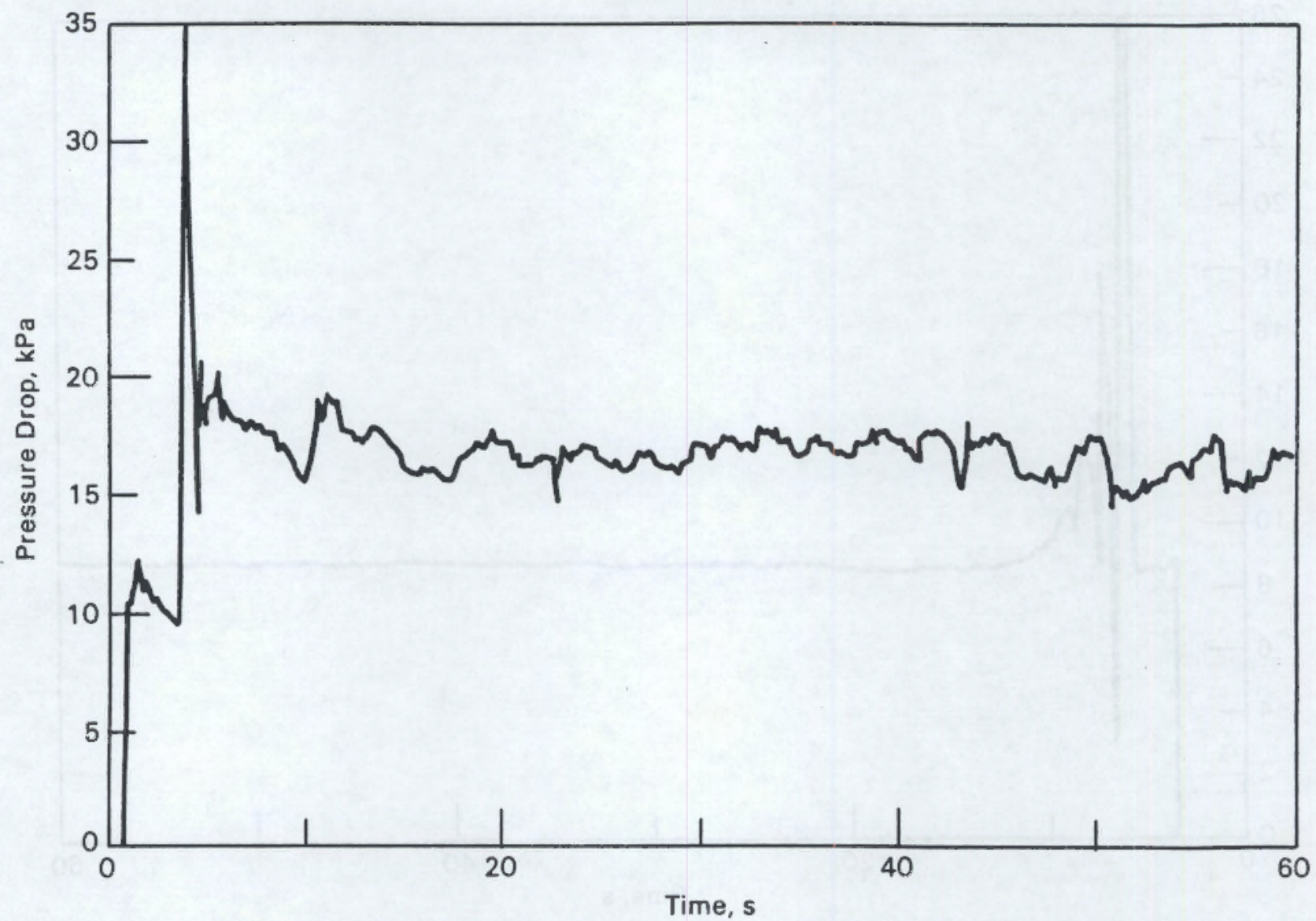

FIGURE 7.7. Pressure Drop Versus Resuspension Time for WV-205 Slurry at a $194 \mathrm{~mL} / \mathrm{s}$ Flow Rate After $20 \mathrm{~h}$ of Settling in 12.7-mm-ID Pipe

is enough to move the settled sludge through as a plug since these pressure drops are much higher than those attained in the 9.52- and 12.7-mm-ID runs.

The behavior of the resuspension curve for the $44-h$ settling time test is quite similar to the curve for the $20-h$ test and indicates no problems with resuspending the $44-h$ settled slurry.

\subsection{RESULTS AND ANALYSIS OF DATA}

The results summarized above indicate that resuspension occurred at all flow rates down to around 10 to $30 \mathrm{~mL} / \mathrm{s}$. These velocities are below those for turbulent flow in the pipe as based on the slurry viscosity and nominal pipe diameter (see Tables 6.4 and 6.5). However, slurry was only flowing above the 


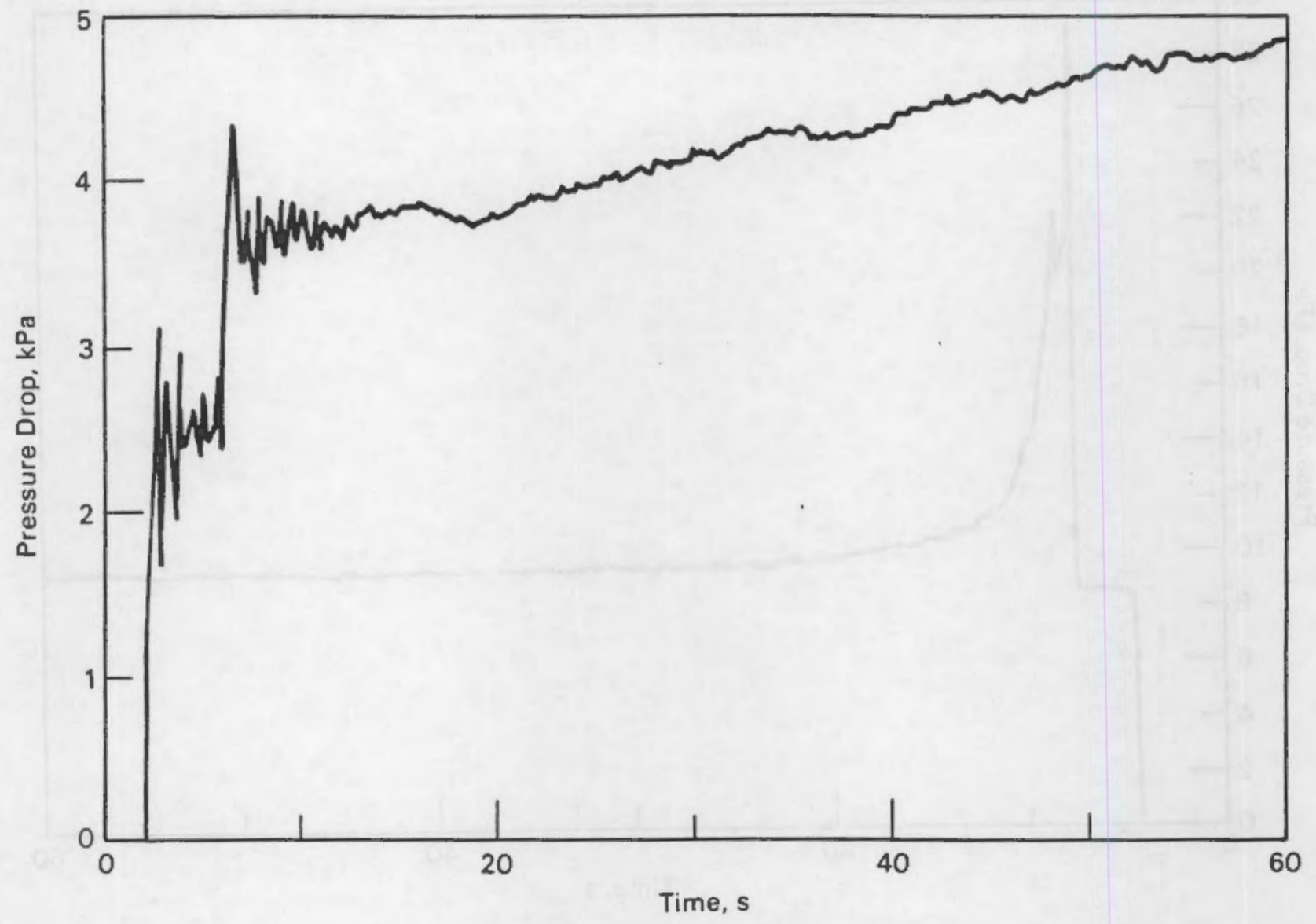

FIGURE 7.8. Pressure Drop Versus Resuspension Time for WV-205 Slurry at a $0.29 \mathrm{~mL} / \mathrm{s}$ Flow Rate After $44 \mathrm{~h}$ of Settling in 12.7-mm-ID Pipe

settled solids, so calculating the velocity based on the pipe diameter may be erroneous. The Reynolds number was recalculated based on the hydraulic radius, the wetted area/perimeter, and the flow through this free area, as shown in Equation (7.1):

$$
\operatorname{Re}\left(\begin{array}{c}
\text { Yield } \\
\text { Pseudoplastic }
\end{array}\right)=2^{3-n}\left(\frac{n}{1+3 n}\right)^{n} D^{n} v^{2-n} \frac{p}{K}
$$

The rheological parameters as determined from the homogeneous transport experiments, are used in Equation (7.1). These include a yield stress of 1.39 Pa, a consistency index of $0.034 \mathrm{~Pa} \cdot \mathrm{s}$, and a flow behavior index of 0.725 


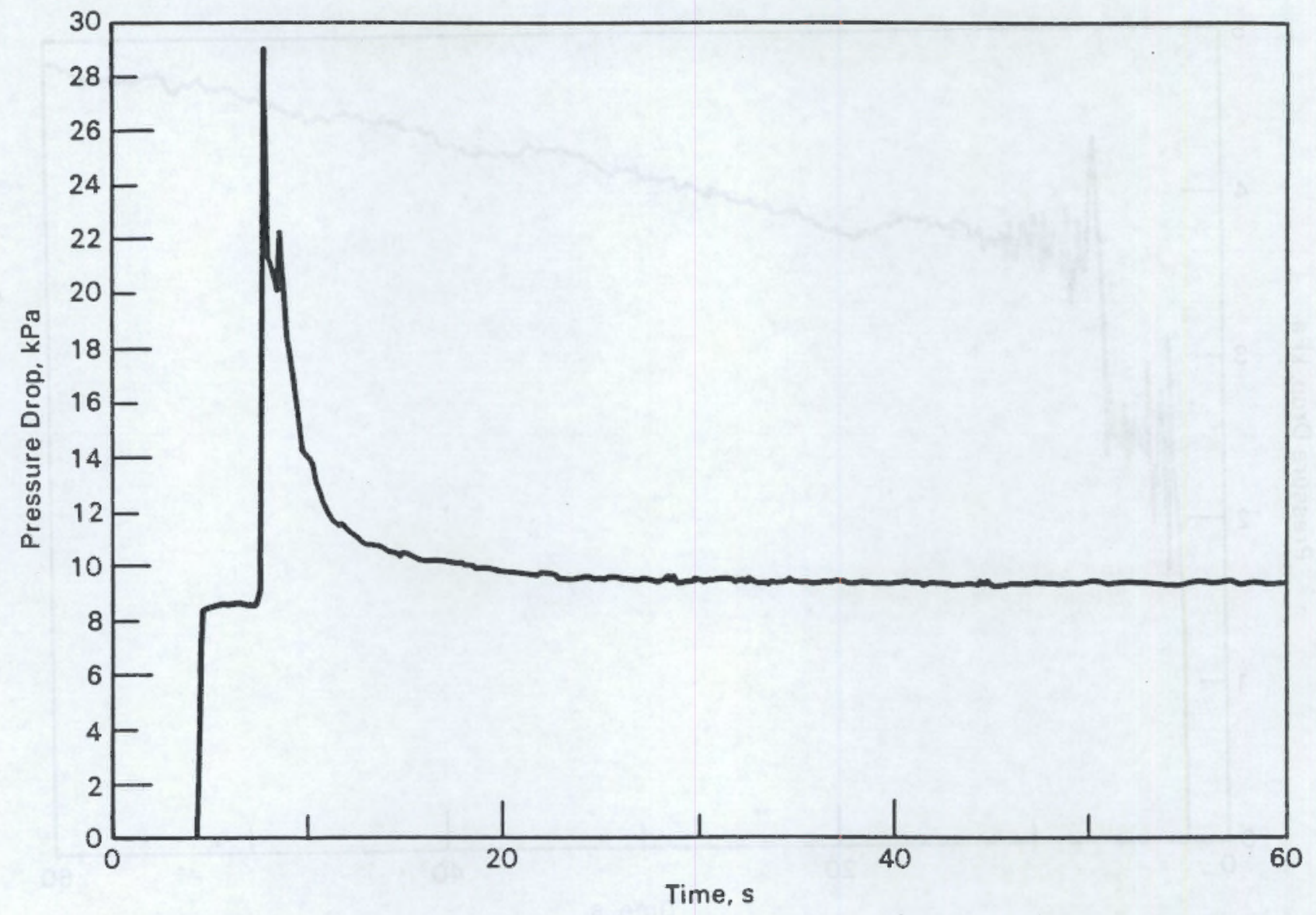

FIGURE 7.9. Pressure Drop Versus Resuspension Time for WV-205 Slurry at a $40 \mathrm{~mL} / \mathrm{s}$ Flow Rate After $44 \mathrm{~h}$ of Settling in 12.7-mm-ID Pipe

(Table 6.5). Tabulated Reynolds numbers appear in Table 7.2. The results based on these Reynolds numbers indicate that suspension in laminar flow is occurring. To confirm this, resuspension experiments were conducted using water to resuspend the settled solids. Resuspension was observed to occur in the laminar region.

Since turbulent activity is not present, another mechanism must result in particle pickup. This mechanism is the lift force due to a velocity gradient across a particle and the drag force due to flow around the particle. The lift force tends to raise the particle from the bed, similar to an airfoil, and the drag force tends to roll it along the bed surface. These two forces vary with the particle area exposed to flow. The forces that resist resuspension are gravity and interparticle attraction. (The interparticle attractive forces are 


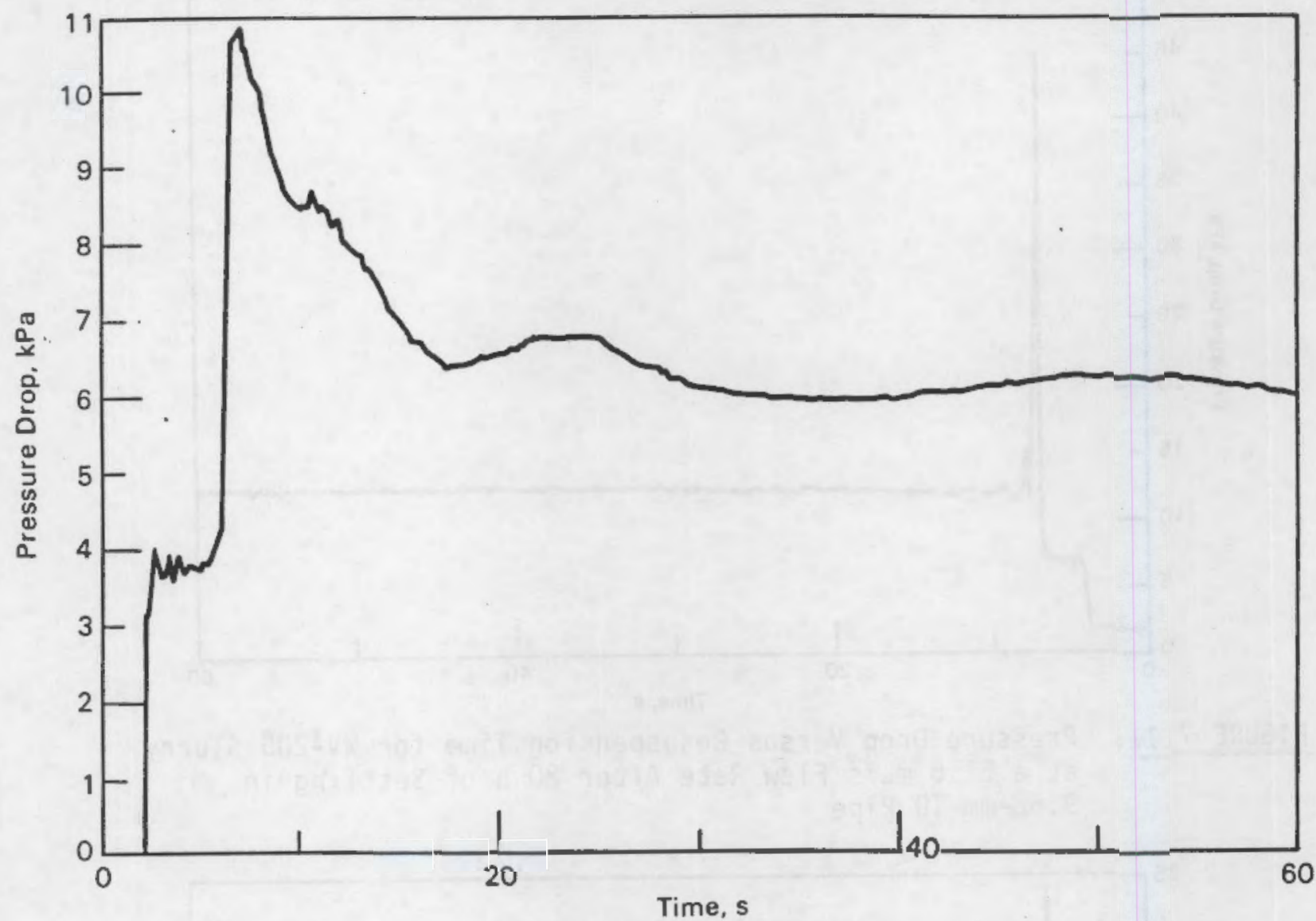

FIGURE 7.10. Pressure Drop Versus Resuspension Time for WV-205 Slurry at a $9.4 \mathrm{~mL} / \mathrm{s}$ Flow Rate After $20 \mathrm{~h}$ of Settling in 9.52-mm-ID Pipe

considered to be small because of the small measured particle sizes, which indicate the absence of flocculation due to the low yield stress of this slurry.) The gravitational force is proportional to the particle volume. The ratio of lift and drag forces to the force of gravity varies with the reciprocal of the particle diameter. As particles become quite small, in the order of $1 \mu \mathrm{m}$, the lift and drag forces become much greater than gravity and may result in particle resuspension. This mechanism is further discussed in the next section. 


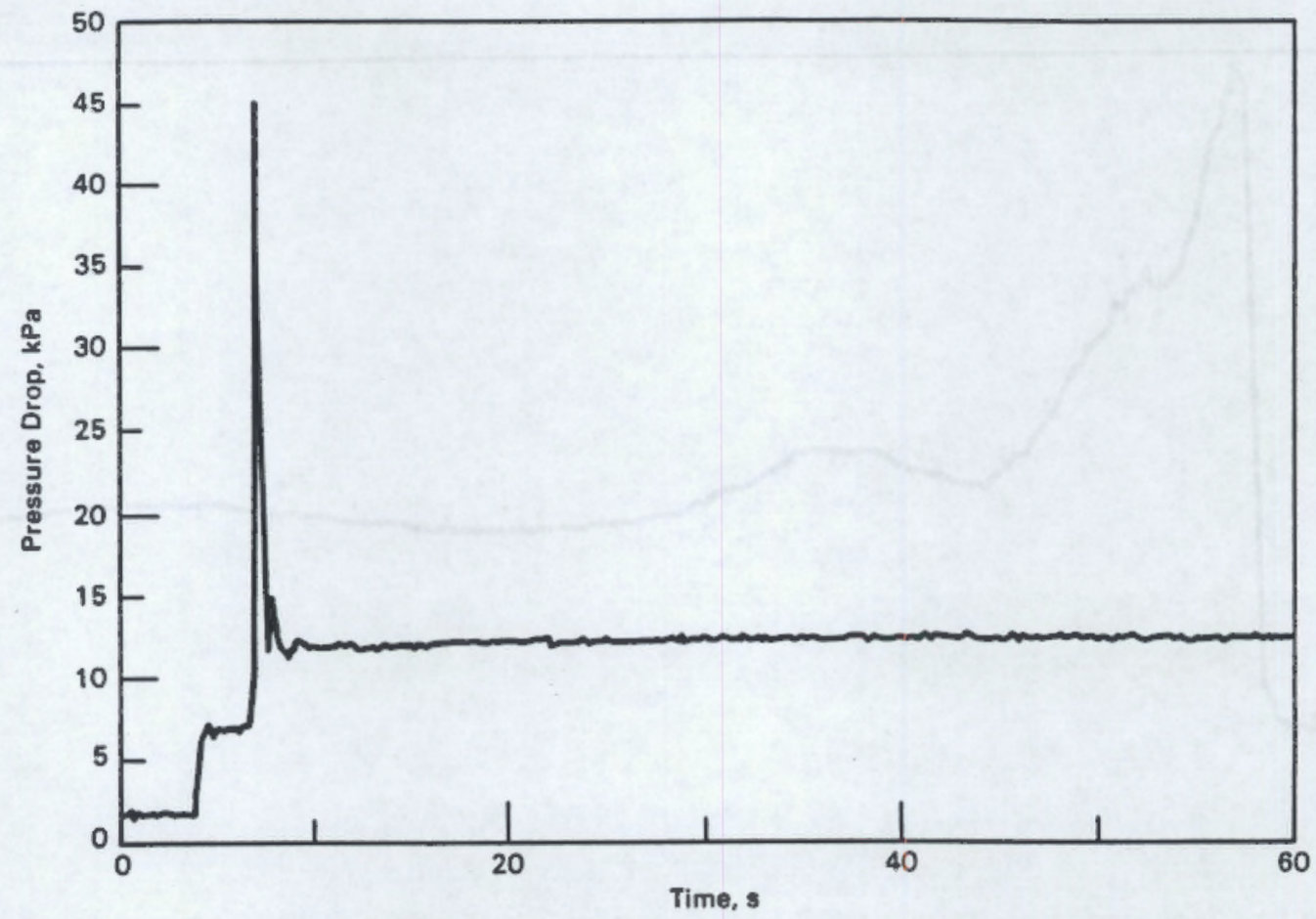

FIGURE 7.11. Pressure Drop Versus Resuspension Time for WV-205 Slurry at a $51.5 \mathrm{~mL} / \mathrm{s}$ Flow Rate After $20 \mathrm{~h}$ of Settling in 9.52-mm-ID Pipe

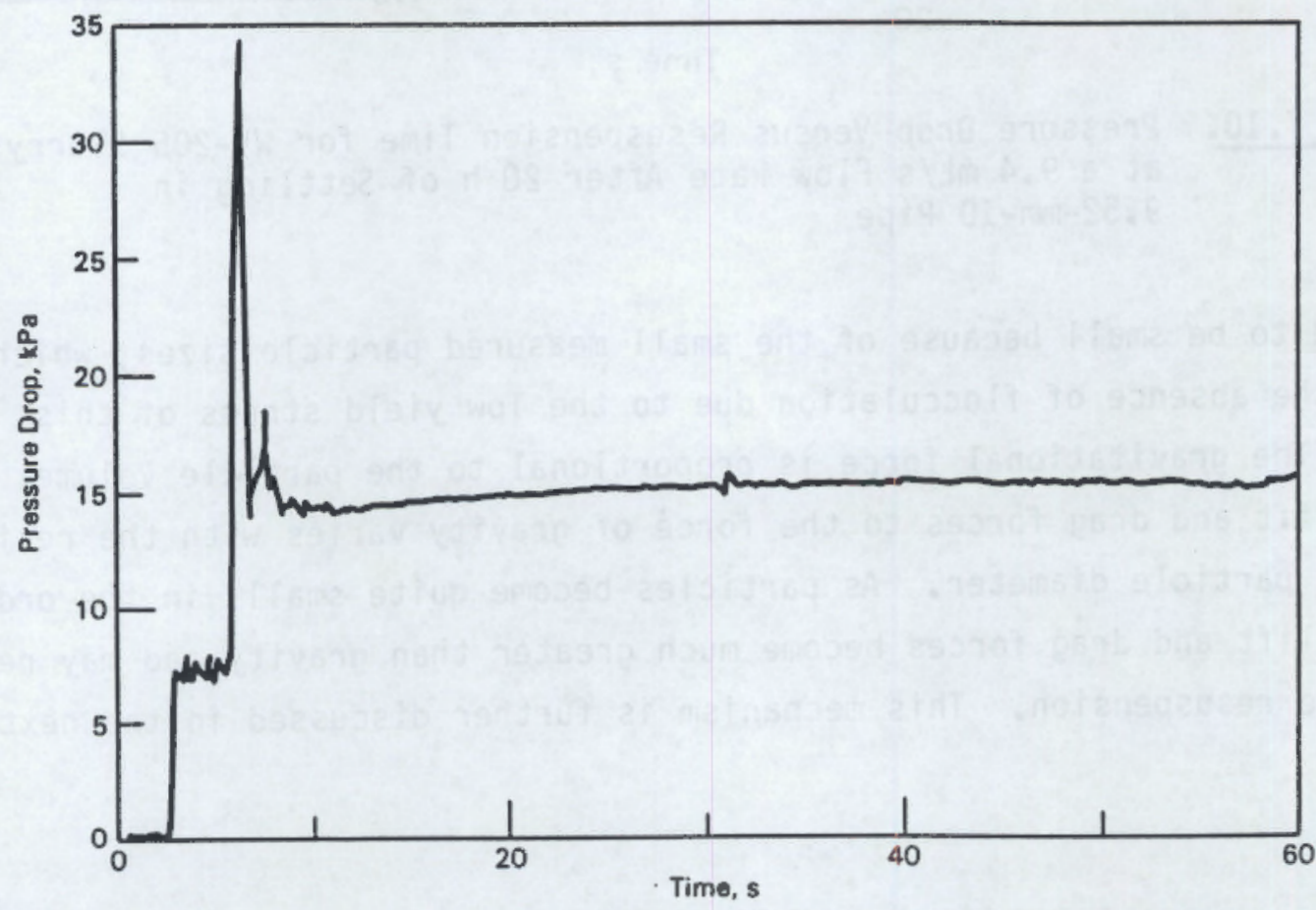

FIGURE 7.12. Pressure Drop Versus Resuspension Time for WV-205 Slurry at a $73.8 \mathrm{~mL} / \mathrm{s}$ Flow Rate After $20 \mathrm{~h}$ of Settling in 9.52-mm-ID Pipe 


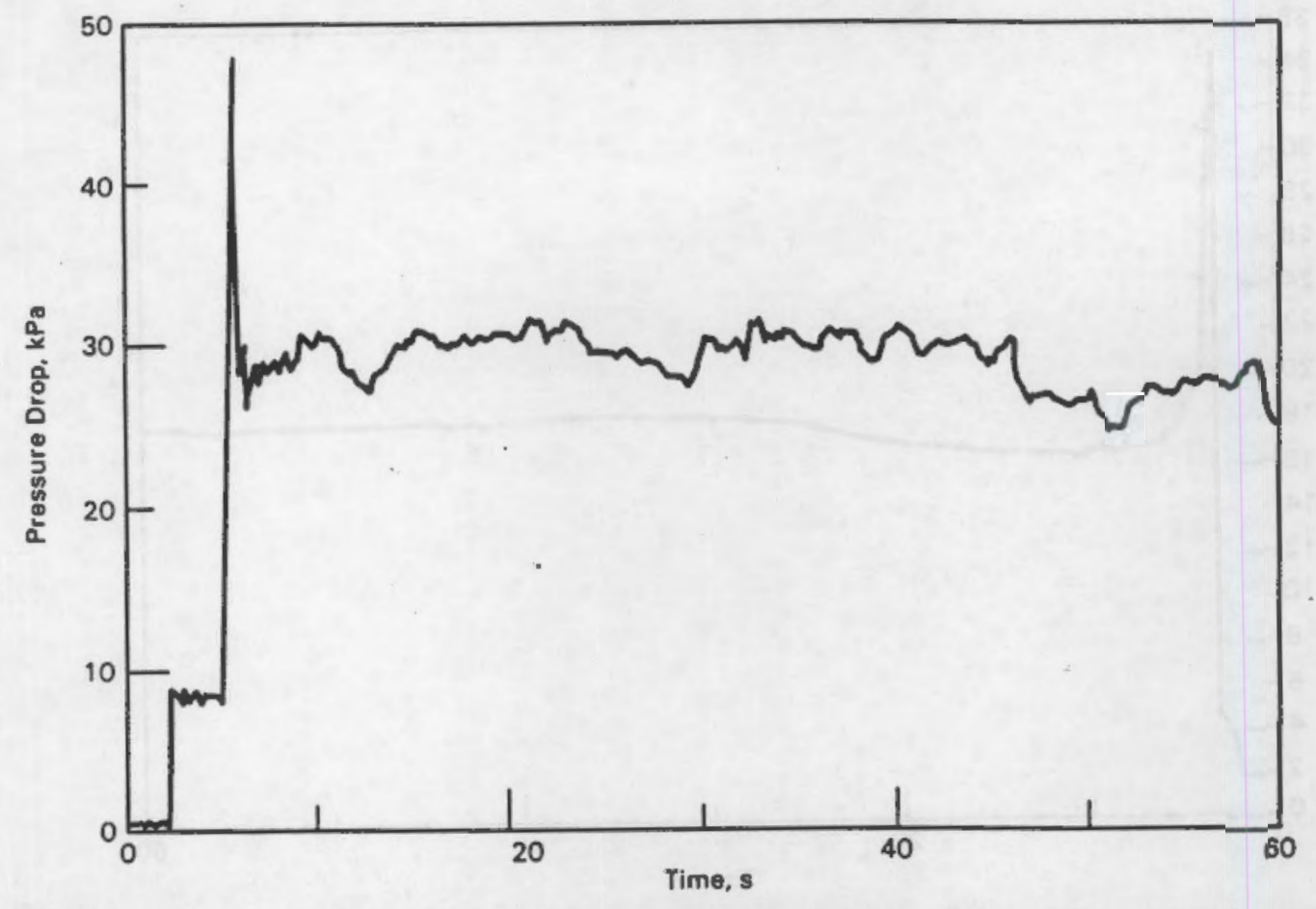

FIGURE 7.13. Pressure Drop Versus Resuspension Time for WV-205 Slurry at a $117 \mathrm{~mL} / \mathrm{s}$ Flow Rate After $20 \mathrm{~h}$ of Settling in 9.52-mm-ID Pipe

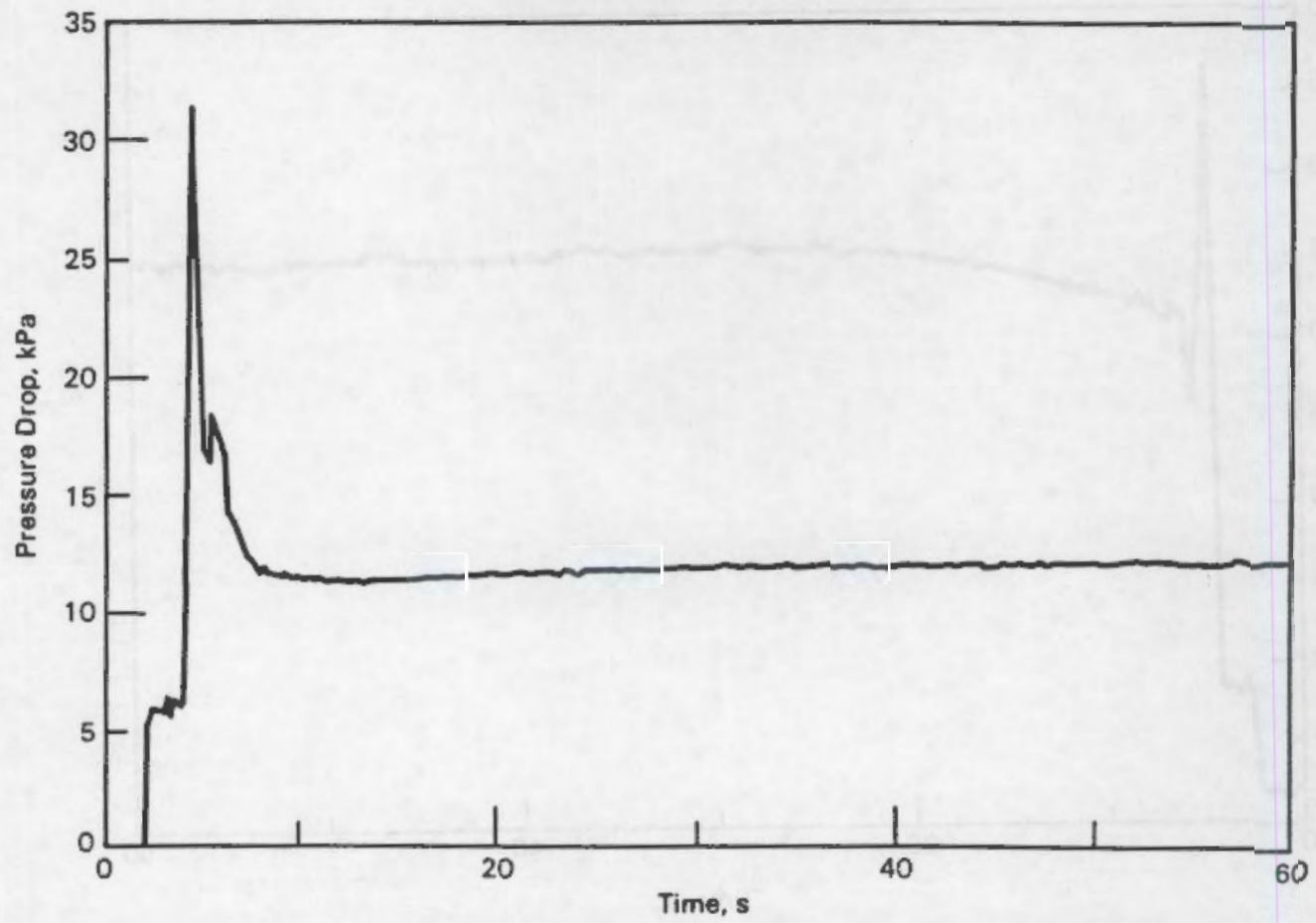

FIGURE 7.14. Pressure Drop Versus Resuspension Time for WV-205 Slurry at $50.1 \mathrm{~mL} / \mathrm{s}$ Flow Rate After $44 \mathrm{~h}$ of Settling in 9.52-mm-ID Pipe 


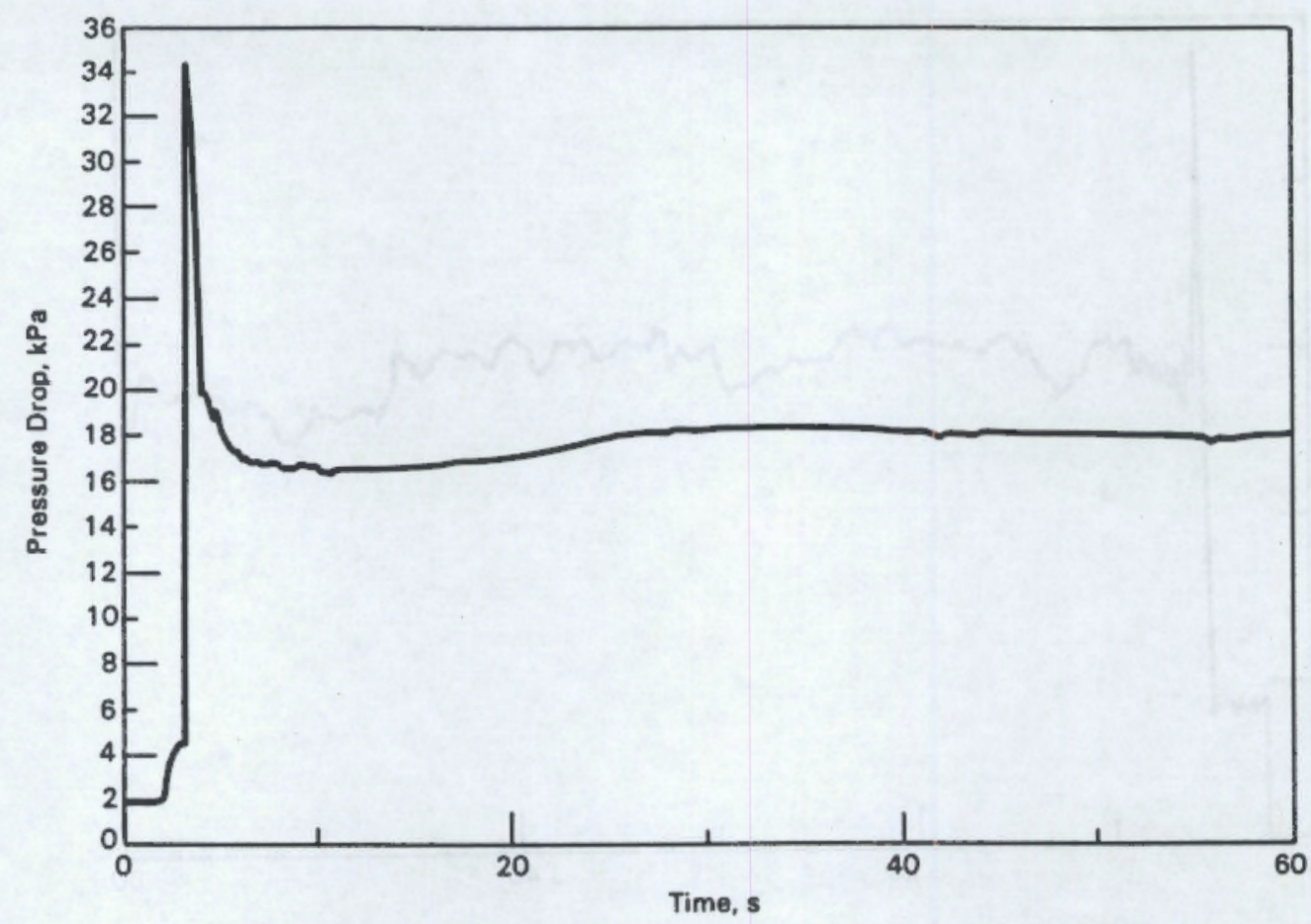

FIGURE 7.15. Pressure Drop Versus Resuspension Time for WV-205 Slurry at a $13 \mathrm{~mL} / \mathrm{s}$ Flow Rate After $20 \mathrm{~h}$ of Settling in 6.35-mm-ID Pipe

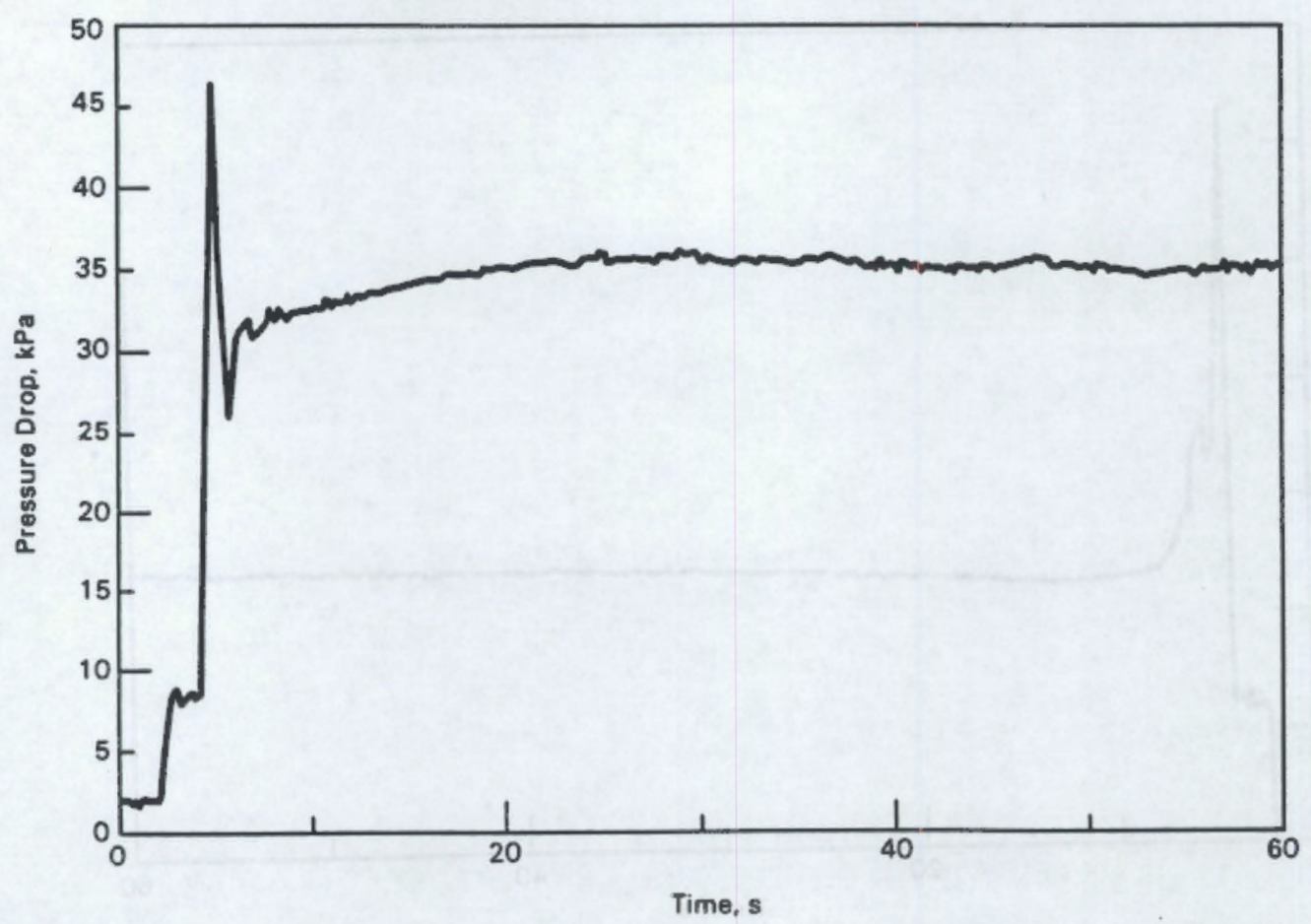

FIGURE 7.16. Pressure Drop Versus Resuspension Time for WV-205 Slurry at a $45.8 \mathrm{~mL} / \mathrm{s}$ Flow Rate After $20 \mathrm{~h}$ of Settling in 6.35-mm-ID Pipe 


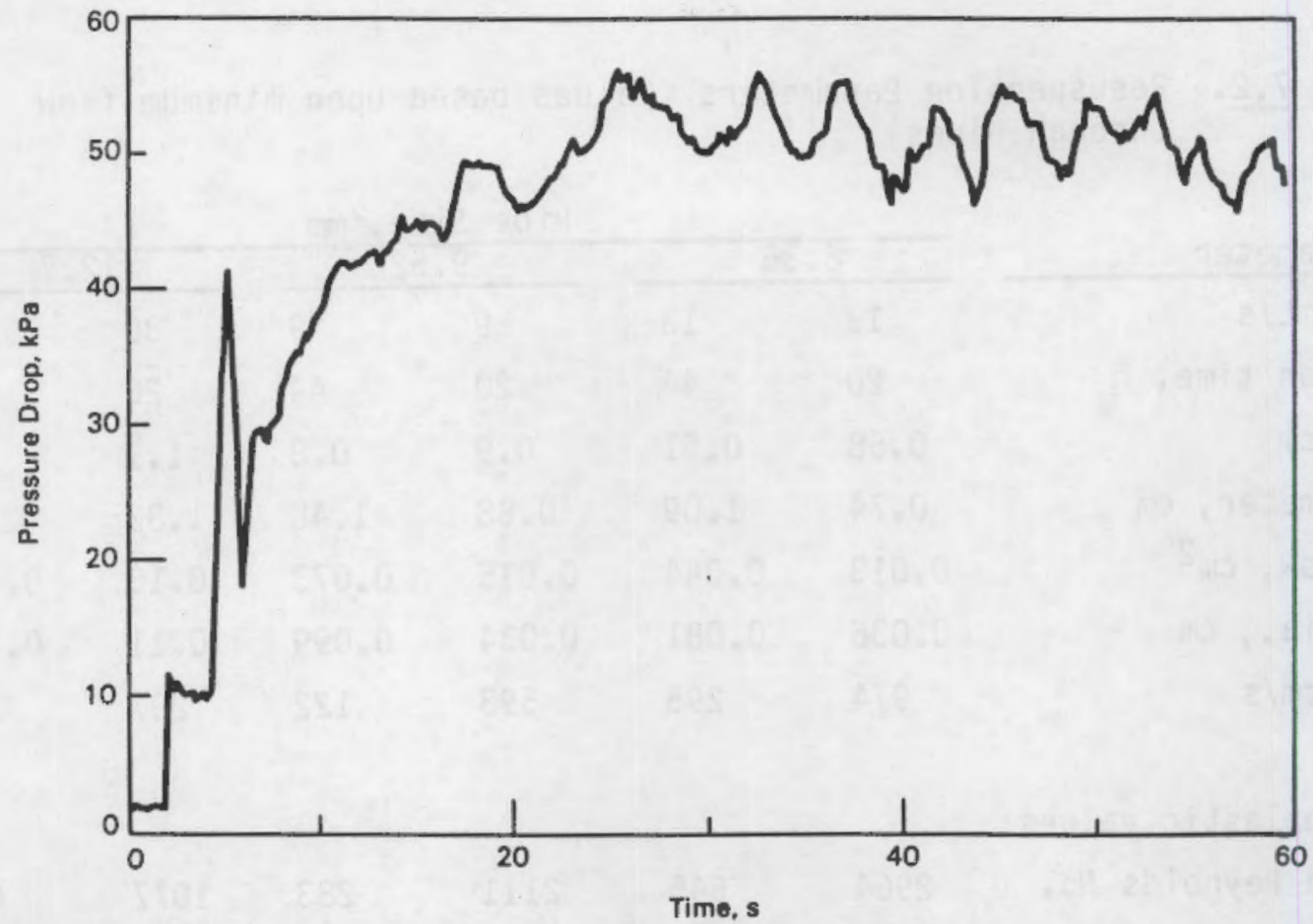

FIGURE 7.17. Pressure Drop Versus Resuspension Time for WV-205 STurry at a $57.5 \mathrm{~mL} / \mathrm{s}$ Flow Rate After $20 \mathrm{~h}$ of Settling in 6.35-mm-ID Pipe

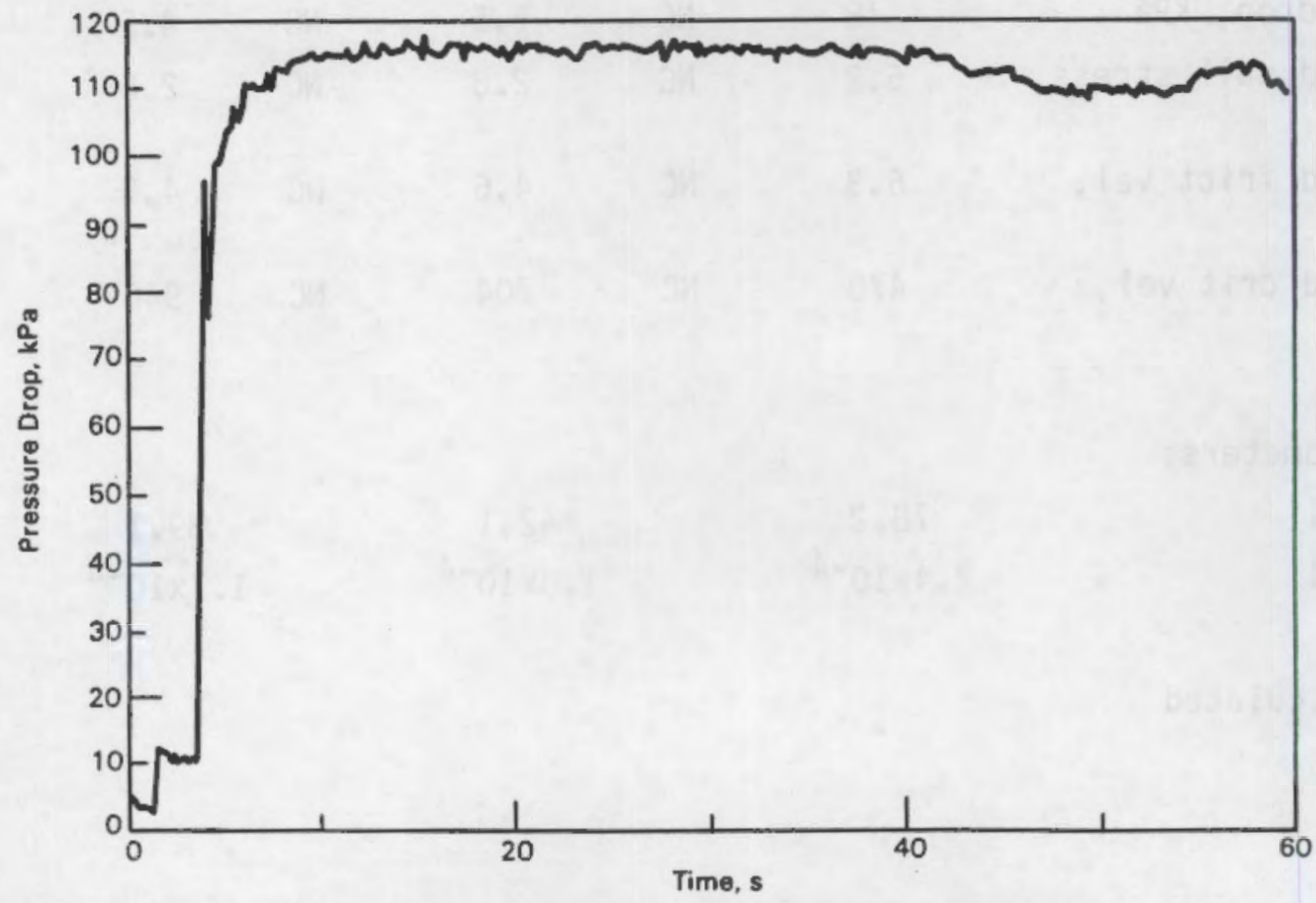

FIGURE 7.18. Pressure Drop Versus Resuspension Time for WV-205 Slurry at a $79.5 \mathrm{~mL} / \mathrm{s}$ Flow Rate After $20 \mathrm{~h}$ of Settling in 6.35-mm-ID Pipe 
TABLE 7.2. Resuspension Parameters (Values based upon minimum flow through pipes)

\begin{tabular}{|c|c|c|c|c|c|c|}
\hline \multirow{3}{*}{$\frac{\text { Parameter }}{\text { Flow rate, } \mathrm{mL} / \mathrm{s}}$} & \multicolumn{6}{|c|}{ Pipe Size, mm } \\
\hline & \multicolumn{2}{|c|}{6.35} & \multicolumn{2}{|c|}{9.52} & \multicolumn{2}{|c|}{12.7} \\
\hline & 13 & 13 & 9 & 9 & 30 & 30 \\
\hline Sedimentation time, $h$ & 20 & 44 & 20 & 44 & 20 & 44 \\
\hline Bed depth, cm & 0.58 & 0.51 & 0.9 & 0.8 & 1.1 & 1.0 \\
\hline Wetted perimeter, $\mathrm{cm}$ & 0.74 & 1.09 & 0.88 & 1.48 & 1.82 & 2.26 \\
\hline Area for $\mathrm{flow}, \mathrm{cm}^{2}$ & 0.013 & 0.044 & 0.015 & 0.073 & 0.10 & 0.197 \\
\hline Hydraulic dia., $\mathrm{cm}$ & 0.036 & 0.081 & 0.034 & 0.099 & 0.11 & 0.174 \\
\hline Flow/area, cm/s & 974 & 295 & 593 & 122 & 297 & 152 \\
\hline
\end{tabular}

Yield pseudoplastic values:

Calculated Reynolds No.

2964

645

2111

283

1077

460

Critical Reynolds No.

2780

2780

3025

$3025 \quad 3250$

3250

Suspension parameters:

Pressure drop, $\mathrm{KPa}$
Calculated wall stress,
$\mathrm{Pa}$
Calculated frict vel,
$\mathrm{cm} / \mathrm{s}$
$\mathrm{Calculated} \mathrm{crit} \mathrm{vel}$,
$\mathrm{cm} / \mathrm{s}$

18 NC

6.5

NC

4.5

NC

5.2 NC

2.8

NC

2.6

NC

$6.3 \quad N C$

4.6

NC

4.5

NC

470

NC

704

NC

940

NC

Shields parameters:

$$
\begin{aligned}
& Y \text { critical } \\
& X \text { critical }
\end{aligned}
$$

NC - not calculated
78.2
$2.4 \times 10^{-4}$

42.1

$1.8 \times 10^{-4}$

39.1

$1.7 \times 10^{-4}$ 


\subsection{COMPARISON OF RESULTS TO OTHER STUDIES}

The results of the slurry transport experiments and the resuspension experiments were compared to the results obtained by other investigators. Section 8.1 discusses the slurry transport experiments. Section 8.2 discusses the slurry resuspension experiments.

\subsection{HOMOGENEOUS SLURRY TRANSPORT EXPERIMENTS}

Figures 8.1 through 8.4 compare the friction factor results obtained in the experimental program with those predicted by the yield power law model for yield pseudoplastic fluids for the three pipe diameters and the replicate series of runs. The theoretical calculations are in good agreement with the experimental results for all three pipe diameters.

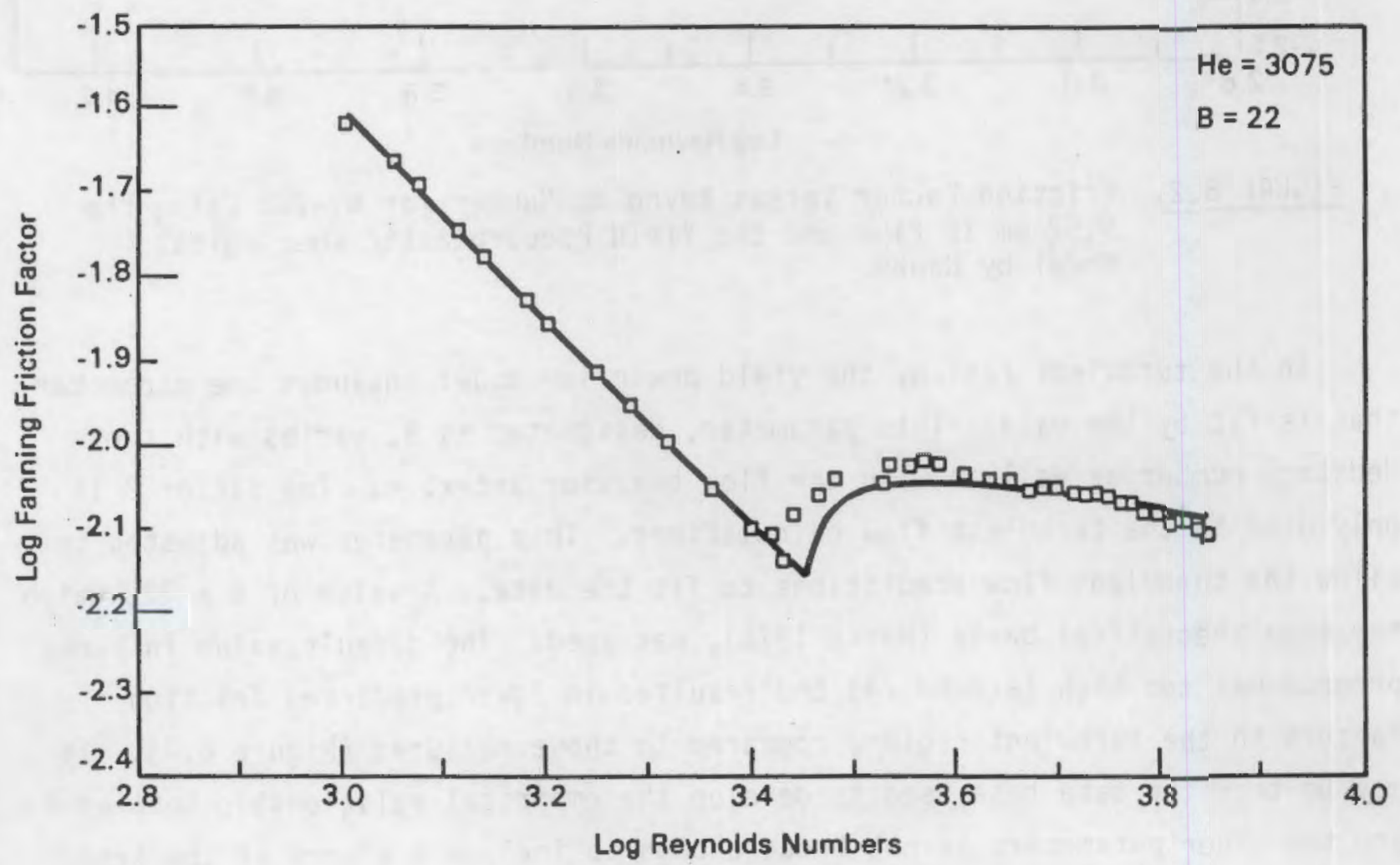

FIGURE 8.1. Friction Factor Versus Reynolds Number for WV-205 Using the 6.35-mm-ID Pipe and the Yield Pseudoplastic Rheological Model by Hanks 


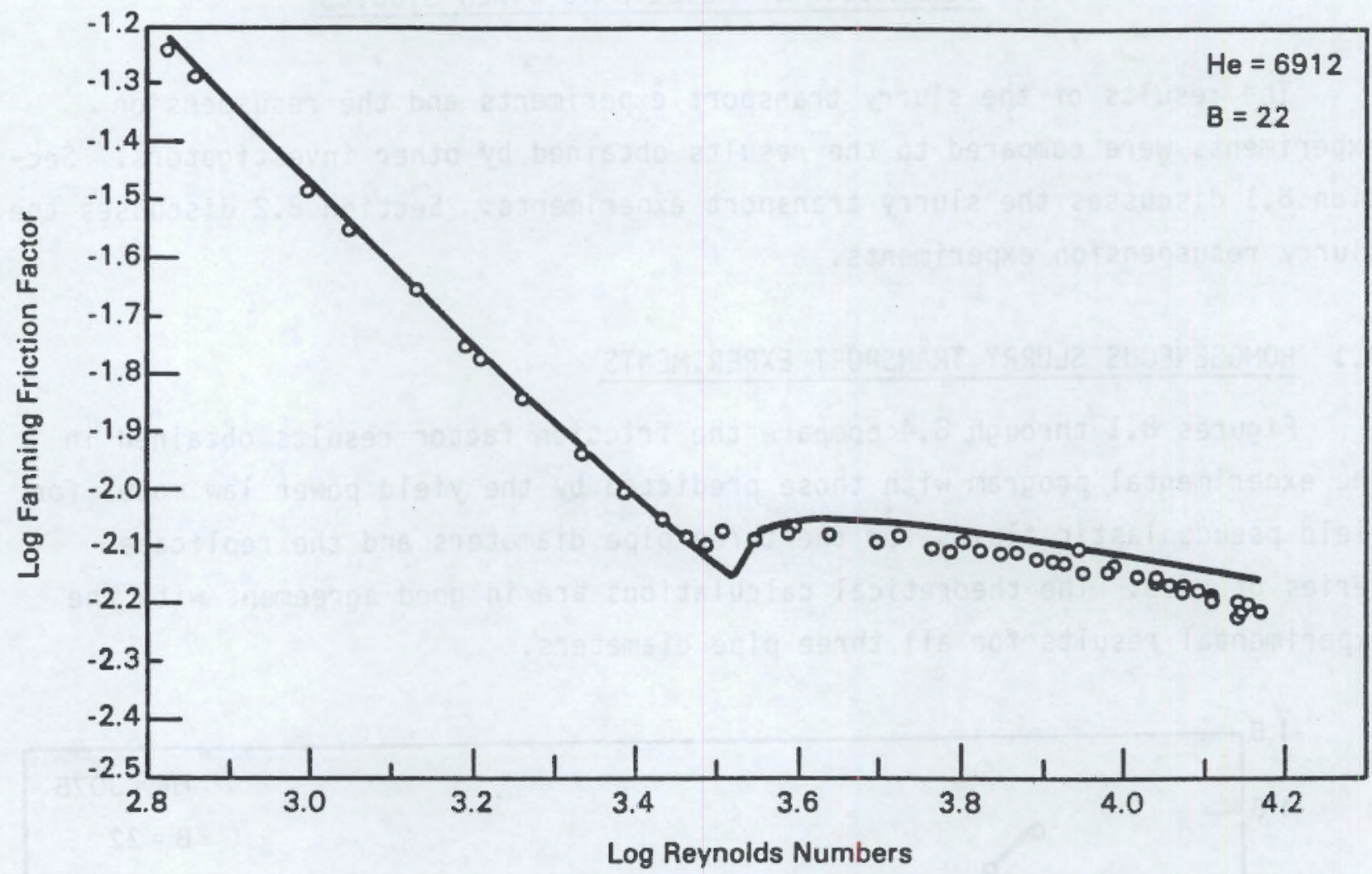

FIGURE 8.2. Friction Factor Versus Reynolds Number for WV-205 Using the 9.52-mm-ID Pipe and the Yield Pseudoplastic Rheological Model by Hanks

In the turbulent region, the yield power law model includes one parameter that is fit by the data. This parameter, designated as $B$, varies with the Hedstrom number as well as with the flow behavior index, $n$. The factor $B$ is only used in the turbulent flow calculations. This parameter was adjusted to allow the turbulent flow predictions to fit the data. A value of $B=22$, which has some theoretical basis (Hanks 1978), was used. The default value in Hanks' program was too high (around 74 ) and resulted in lower predicted friction factors in the turbulent region, compared to those measured (Figure 8.4). It may be that the data base used to develop the empirical relationship between $B$ and the other parameters is not broad enough to include a slurry of the type used in this study. Further work should be conducted to generate a correlation for $B$ for the slurries encountered in waste processing. 


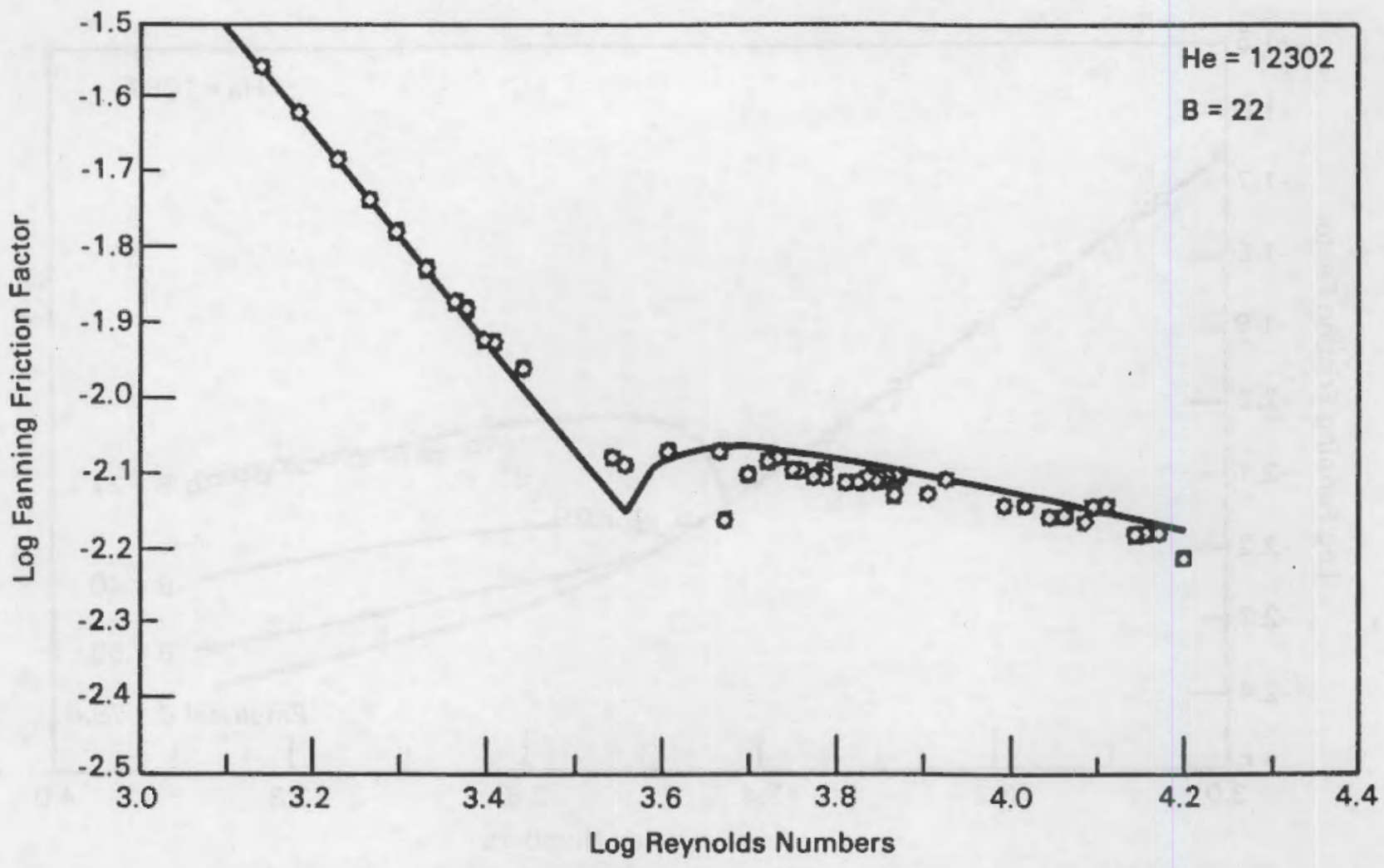

FIGURE 8.3. Friction Factgr Versus Reynolds Number for WV-205 Using the 12.7-mm ${ }^{-10}$ Pipe and the Yield Pseudoplastic Rheological Model by Hanks

An alternative equation sometimes used to correlate turbulent flow of nonNewtoninan fluids is the Torrance equation (Torrance 1963), which is based upon Prandtl's mixing length theory. The equation assumes that turbulent stresses are absent in a laminar layer near the pipe wall and that viscous stresses are absent in the turbulent core of the pipe. The equation is implicit in terms of the friction factor.

The Torrance equation states:

$$
\begin{aligned}
1 / f= & \frac{2.69}{n}-2.95+\frac{4.53}{n} \log \left[1-\frac{\tau_{y}}{\tau_{w}}\right] \\
& +\frac{4.53}{n} \log \operatorname{Re}_{t} f^{2-m}+\frac{0.68}{n}(5 n-8)
\end{aligned}
$$




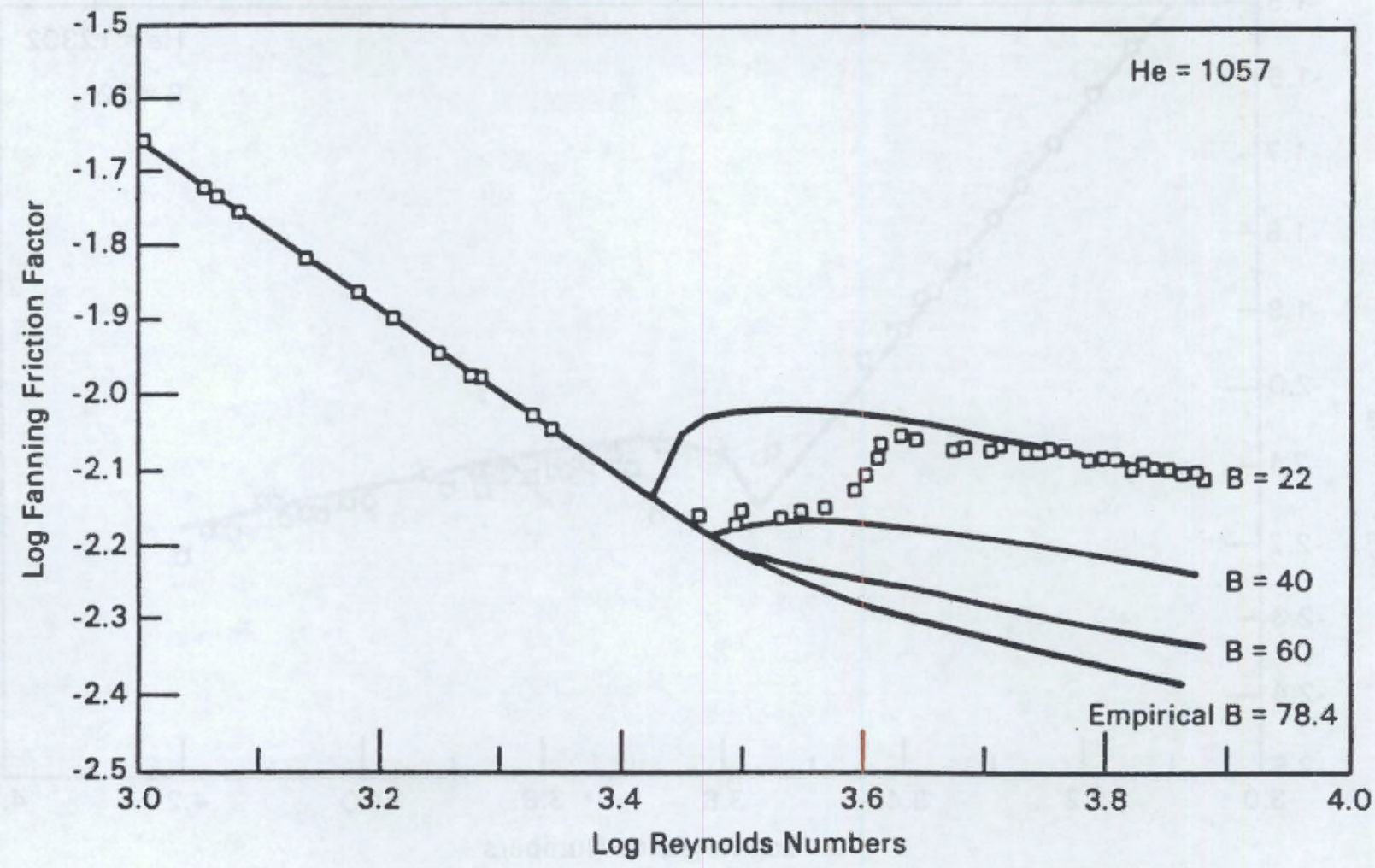

FIGURE 8.4. Friction Factor Versus Reynolds Number for the WV-205 Slurry with the 6.35-mm-ID Pipe. The yield pseudoplastic model was fit to the data using various $B$ values.

where $R e_{t}=$ Torrance Reynolds number $=\frac{D^{n} v^{2-n_{\rho}}}{K 8^{n-1}}$

Figures 8.5 through 8.7 indicate fair agreement of the results with the Torrance equation. Hanks' model with an adjustable B value of 22 correlates better with the results, however.

\subsection{SLURRY RESUSPENSION EXPERIMENTS}

The mechanisms for particle resuspension include lift and drag forces in addition to turbulence. For the experiments on resuspension, resuspension was observed in the test loop under laminar flow conditions, which indicates that the lift and drag forces may be sufficient to resuspend the particles. Kao and Wood (1974) present a mathematical model for the mean pipe velocity required to obtain resuspension, $v_{c}$, for particles under lift: 


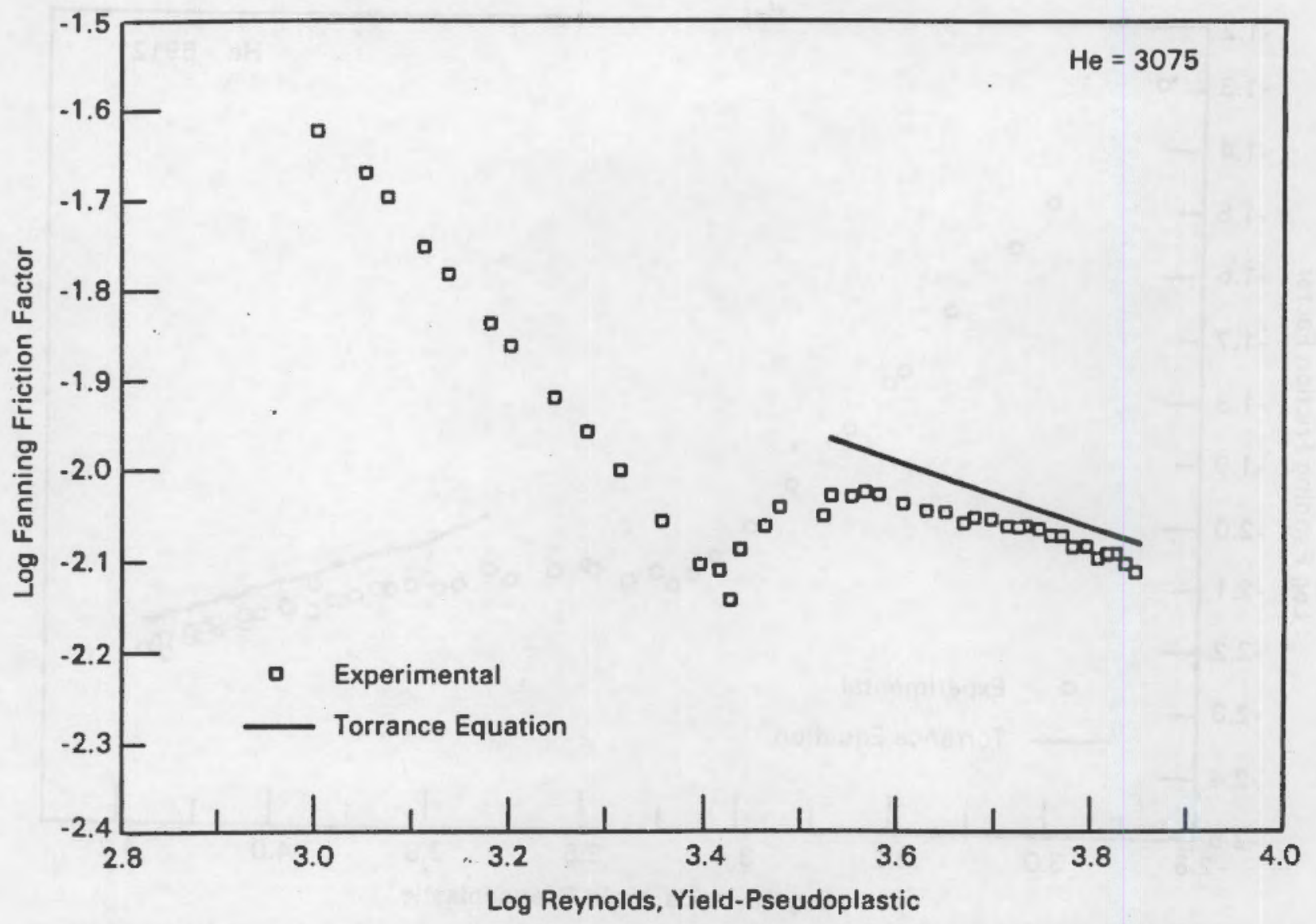

FIGURE 8.5. Friction Factor Versus Reynolds Number for WV-205 Slurry with the 6.35-mm-ID Pipe and Fit to the Torrance Equation

$$
V_{c}=\underset{\text { velocity }}{\text { critical }}=\sqrt{\frac{4 g(s g-1) d}{3 a \beta^{2}(d / D)^{2 / s}}}
$$

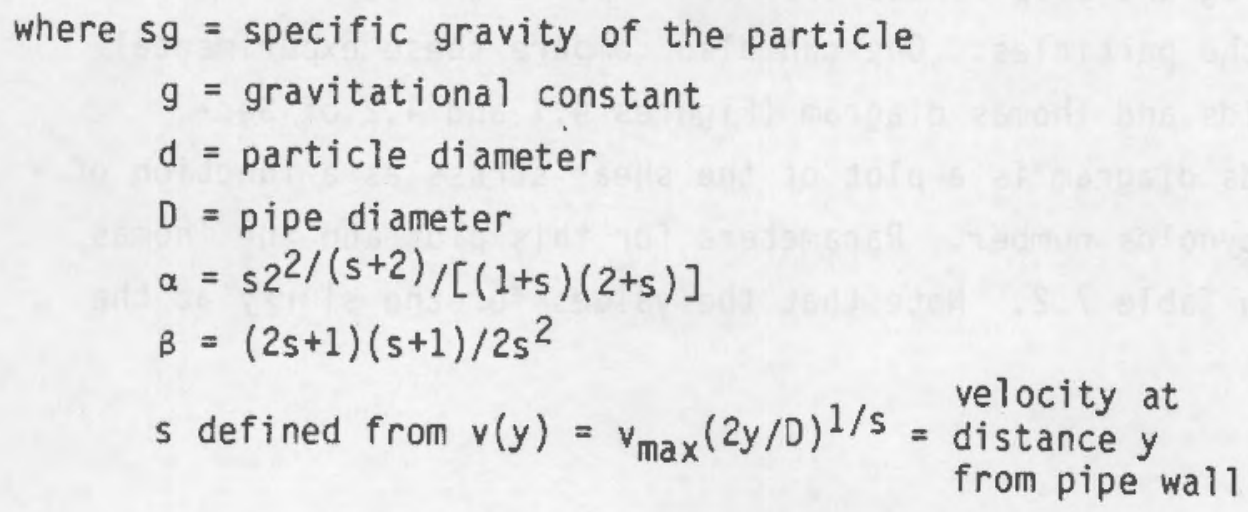




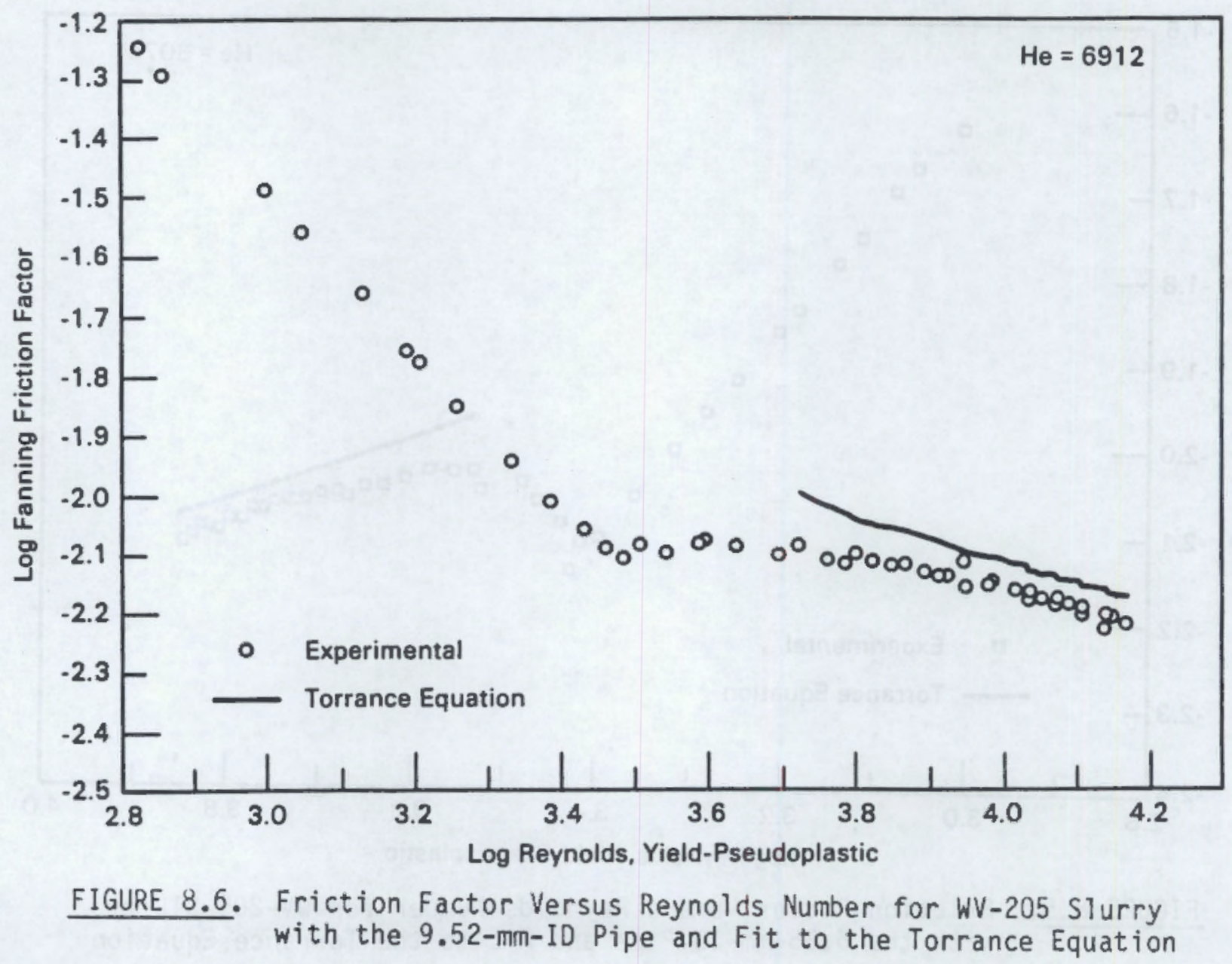

Calculations for this velocity--assuming a particle density of $2 \mathrm{~g} / \mathrm{cm}^{3}$, a radius of $10 \mu \mathrm{m}$ for the particle, and a value of $s=1$ for laminar flow--are summarized in Table 7.2 in Section 7.3. Note that these velocities are generally higher than the velocities calculated based upon the free area for flow. This indicates that drag and slip forces are more important than turbulent forces in suspending the particles. One can also compare these experimental results with the Shields and Thomas diagram (Figures 4.1 and 4.2 of Section 4.0). The Shields diagram is a plot of the shear stress as a function of a friction velocity Reynolds number. Parameters for this plot and the Thomas plot are summarized in Table 7.2. Note that the values for the slurry at the 


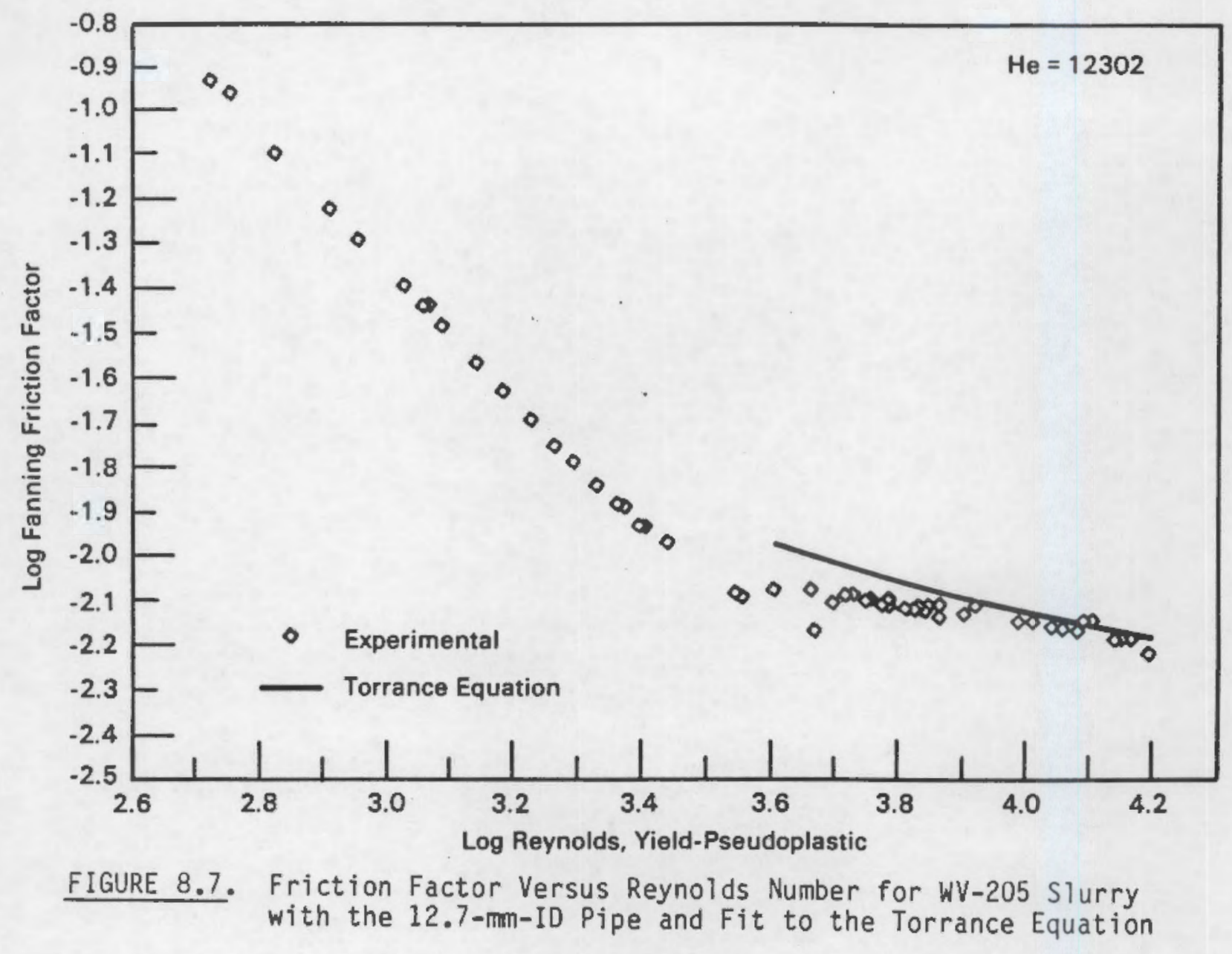

lowest flows observed indicate that resuspension should occur since $Y_{\text {calc }}$ appears larger than the $Y_{c r}$ values on the Shields plot. The slurry values are not within the range of either diagram, however, so it is difficuit to conclude what these diagrams predict about this particular slurry. 



\subsection{COMPARISON OF LABORATORY TO PILOT SCALE RESULTS}

At first glance, the reported coefficients from the Haake rotational viscometer do not agree well with those from the pipe loop experiment. (Compare data in Section 5.2.2 and Tables 5.4 and 5.8 with those in Tables 6.3 and 6.4.) The Haake viscometer rheological parameters, $n$ and $K$, are determined by performing a linear regression analysis on a logarithmic plot of shear stress (minus yield stress) versus shear rate. The resulting statistics for the regression are reasonable. A nonlinear curve fit on the same data, however, yields different rheological parameters. (The nonlinear curve fit method was used to determine the rheological parameters from the pipe loop experiments.) The nonlinear curve fit values also yield a lower sum of squares of the error than do the parameters determined from the linear regression. Indeed, if the linear regression parameters are used as starting values for the nonlinear curve fit method, the method converges to the same values that it did in the absence of these as starting values. This indicates that there are probably no local minimums in the optimization space and that the true minimum is found by the nonlinear curve fit method. Statistically, this method should be used.

One explanation for the difference between the Haake viscometer and the pipe loop may be wall slip. The results for pipe flow displayed on Figure 6.18 indicate that wall slip is not occurring. A check was made using the method of Skelland (1967), in which the flow/[(stress)(pipe radius ${ }^{3}$ )] is plotted as a function of stress. Figure 9.1 depicts these results and shows a continuous curve for all three pipe data. This indicates the absence of wall slip. Similar conclusions about the absence of wall slip were found for the Haake and capillary tube results (Section 5.2 .2 and 6.4.1).

Perhaps the quandry lies in the use of a small number of data points to fit a three parameter model. Hanks and Hanks (1984) indicated that they take 50 or more data points over a range of shear rates of two orders of magnitude, from 20 to $2000 \mathrm{~s}^{-1}$ for example. Darby (1984) in the same discussion recommends three or more orders of magnitude. These comments emphasize the 


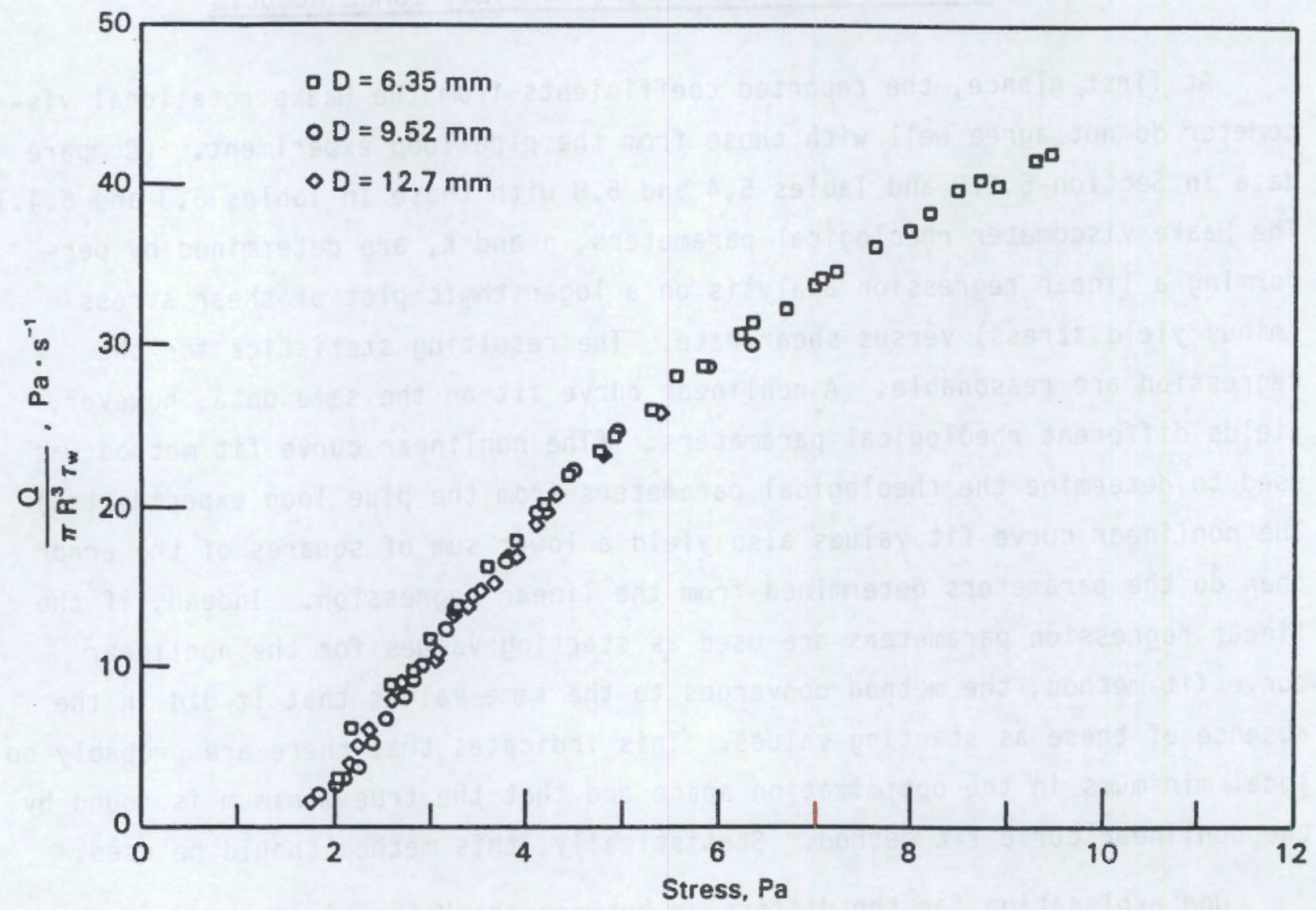

FIGURE 9.1. Evaluation of Wall Slip in the Pipe Loop

empirical nature of these rheological models and limit their validity to allowing only interpolation, not extrapolation, which many researchers say is dangerous (Hanks 1984).

Figures 9.2 and 9.3 compare the Haake data for the three gap sizes to the pipe flow data for the two series of runs. (Raw data from the Haake runs are compared to pipe loop data that include the Mooney-Rabinowitsch correction for shear rate.) These figures indicate that the small gap width data for the Haake agrees fairly well with the pipe flow results over the range of similar shear rates. The larger gap size data do not agree as well, which indicates the necessity to correct these data (Section 5.2.2). An attempt at doing so with the Reiner-Rivlin equation was not satisfactory due to the cylinder stress falling below the yield stress for a portion of the shear rate range. The figures also show that the Haake has a limited shear rate range compared to the 


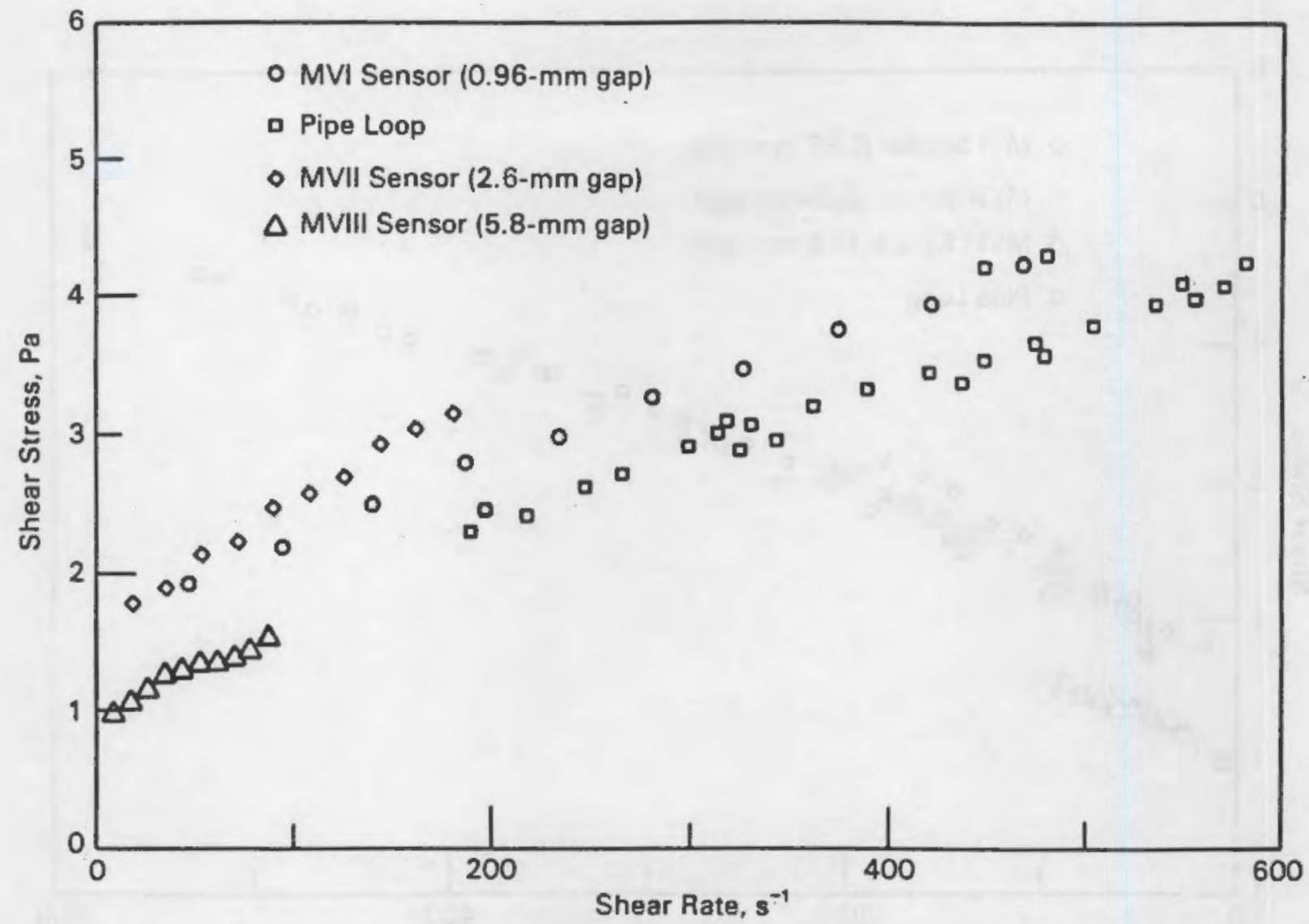

FIGURE 9.2. Comparison of Shear Stress Versus Shear Rate for the Haake Viscometer Using Three Different Sensors and Initial Pipe Loop Data

range for the pipe loop. The reason for the discrepancy in the rheological parameters as determined from the Haake and pipe loop lies then in the range of data available for statistical analysis. The pipe loop has a much wider range and should yield a model appropriate for a wider range of shear rates.

The capillary tube constants, however, appear to agree somewhat better with the pipe loop values (see Table 6.2, 6.3, and 6.4). However, when one uses the various rheological constants and plots the shear stress versus shear rate for the rotational viscometer, the capillary tube, and the pipe loop, little difference is seen between the rotational viscometer predictions at the low shear rates, but large differences are seen between the predictions based on the capillary tube parameters and those of the pipe loop. The capillary tube predictions are about $35 \%$ higher than those of the pipe loop and 


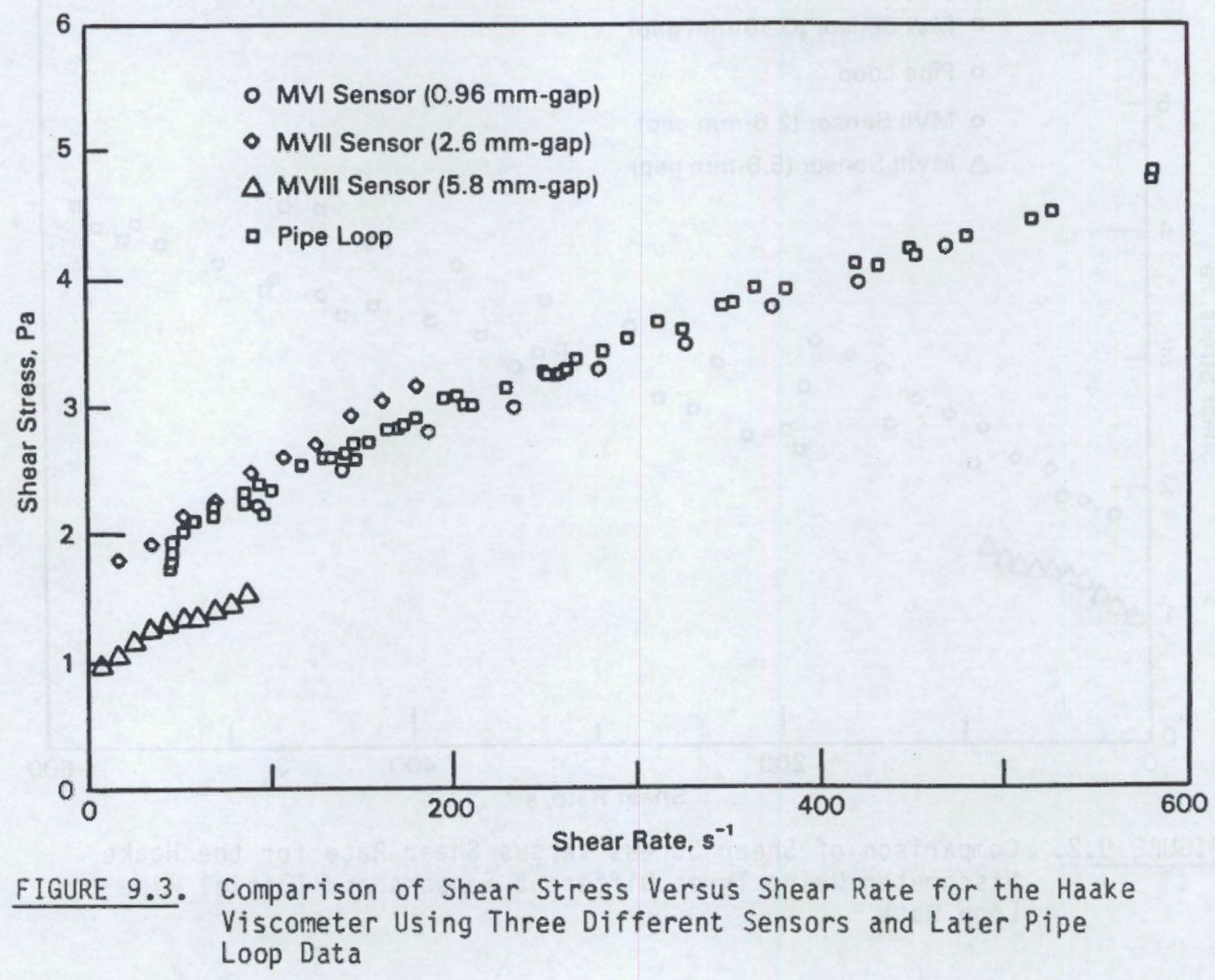

rotational viscometer (see Figure 9.4). This difference may be due to a build up of solids in the stainless steel tubes of the capillary tube and an increase in the viscosity of the slurry due to dissolved air. 


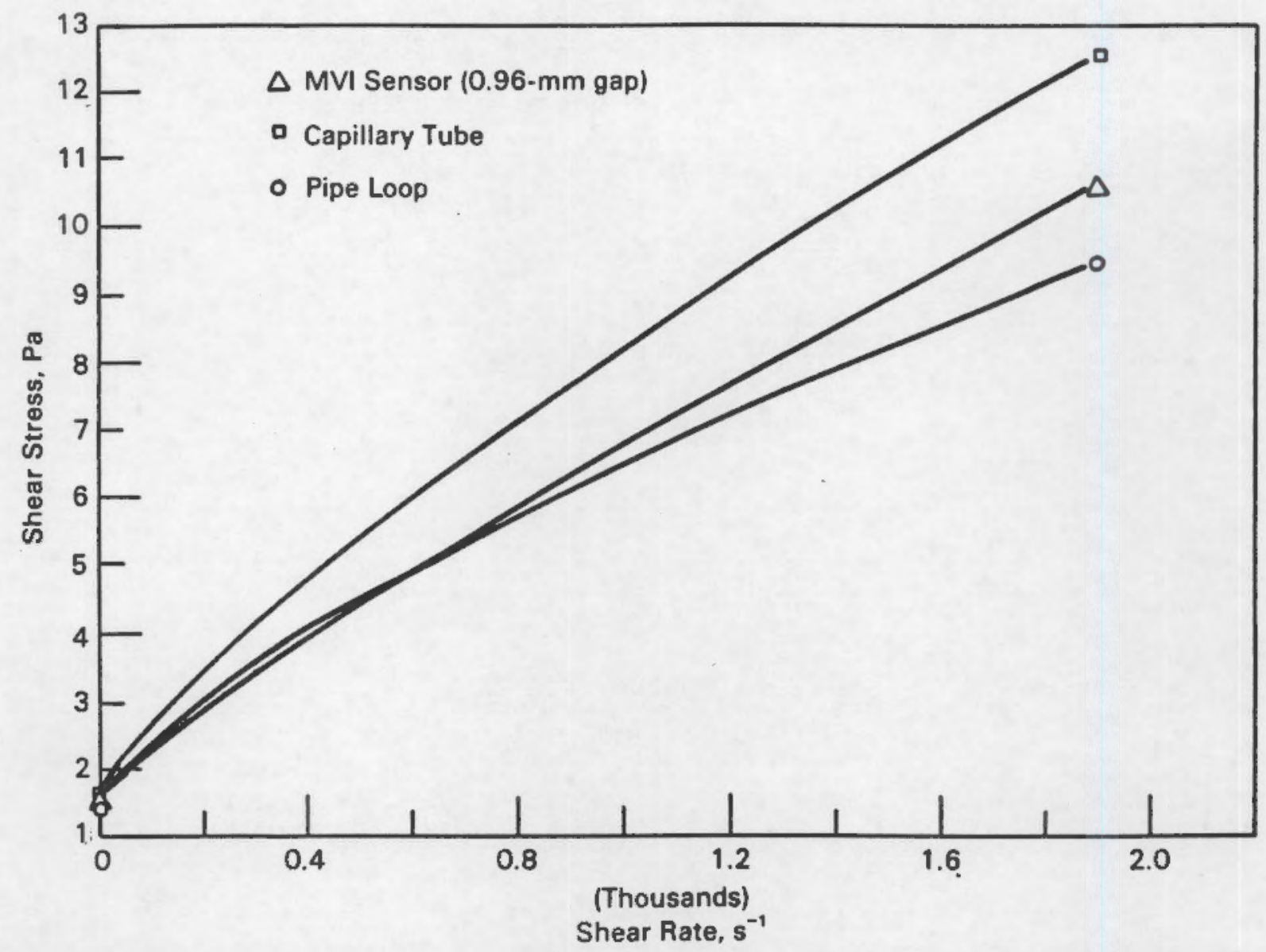

FIGURE 9.4. Comparison of Shear Stress Versus Shear Rate as Determined by the Rotational Viscometer, Capillary Viscometer, and Pipe Loop 


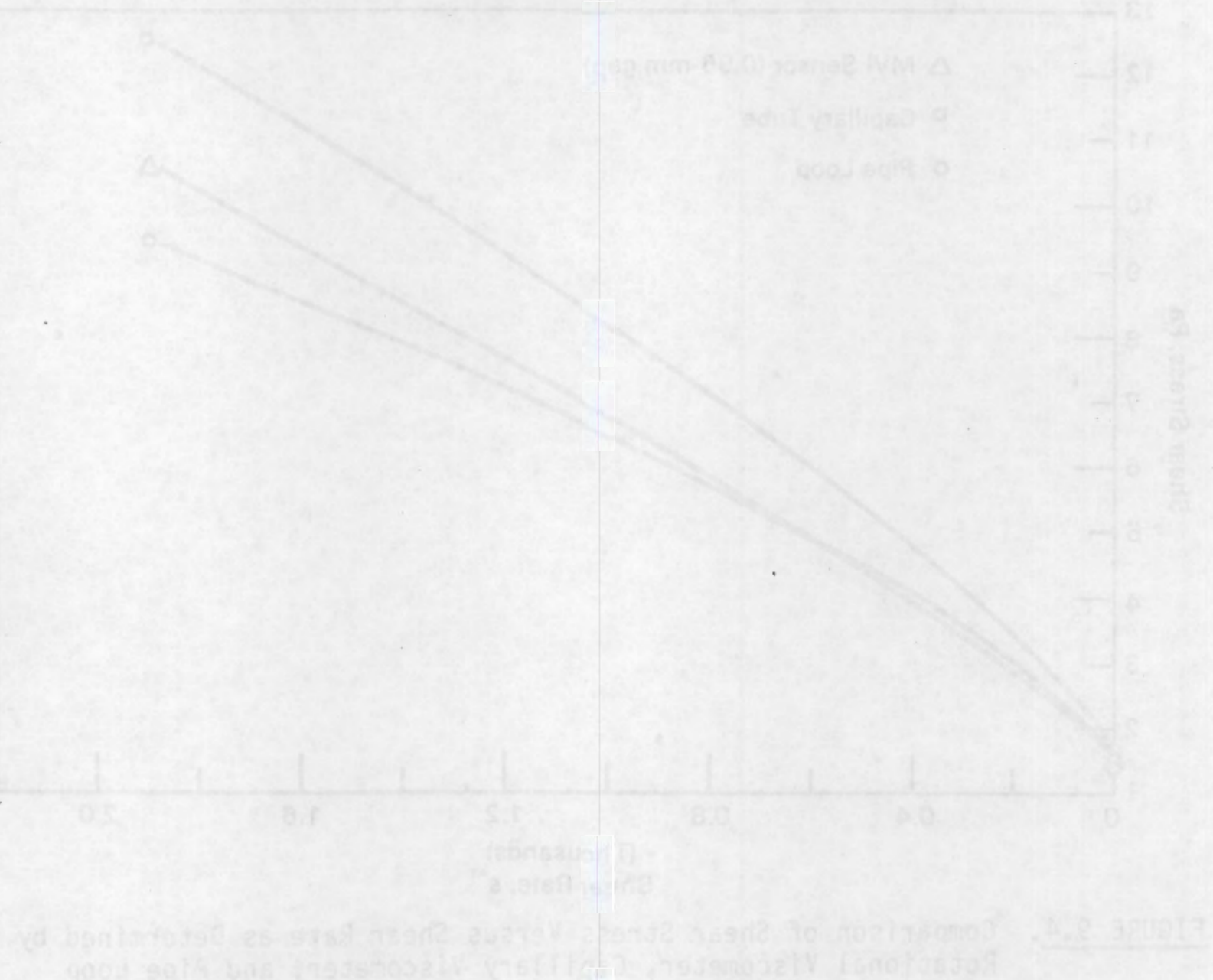

4 


\subsection{RAMIFICATIONS OF RESULTS TO DESIGN OF FULL-SCALE SYSTEMS}

Scale-up of the results to other pipe sizes is treated at length by Carleton and Cheng (1977). Scale-up becomes a problem when extrapolating small pipe data to much larger pipes, especially if the slurry is heterogeneous. For laminar flow of homogeneous slurries that do not settle fast (i.e., the slurry evaluated in this research), Carleton and Cheng recommend using a rheological model such as Bingham plastic or a stress versus shear rate diagram to scale up to a larger pipe diameter. For turbulent flow, a generalized form of the von Karmen equation is used with limited success. In some cases, Bowen's method is used for turbulent flow. This method is based upon a modification of the Blasius equation:

$$
D^{X} \tau_{W}=k Q^{W}
$$

A plot of shear rate, $\tau_{w}$, versus flow rate, $Q$, yields the coefficient, $w$, from which $\tau_{W} / Q^{W}$ versus pipe diameter, $D$, can be plotted to determine $k$ and $x$. Bowen found that this method yielded good results in correlating data from 27-, 35-, and 53-mm-ID pipe results, but it failed to correlate the results for a 329-mm-ID pipe. The use of Bowen's method is illustrated in Figures 10.1 and 10.2, where the turbulent flow data from the water runs and the replicate slurry runs, respectively, are correlated. This method appeared to correlate the data well for the three pipe diameters and can be scaled up for use with the turbulent flow data. It is recommended that this scale-up procedure be checked by using two pipe sizes, the 6.35- and 9.52-mm diameter for instance, to determine the coefficients. Then these coefficients would be used to check the predictions for the third pipe size, the 12.7-man diameter.

The prediction of the transition Reynolds number from laboratory data can be done by a variety of rheological models. The rheological data appeared to fit a yield pseudoplastic model best, so this model will be used to predict the transition Reynolds number. The method of Hanks' (1984) was used in which the transition Reynolds number depends on the three rheological parameters as well as the Hedstrom number. Thus, the different pipe sizes will have different 


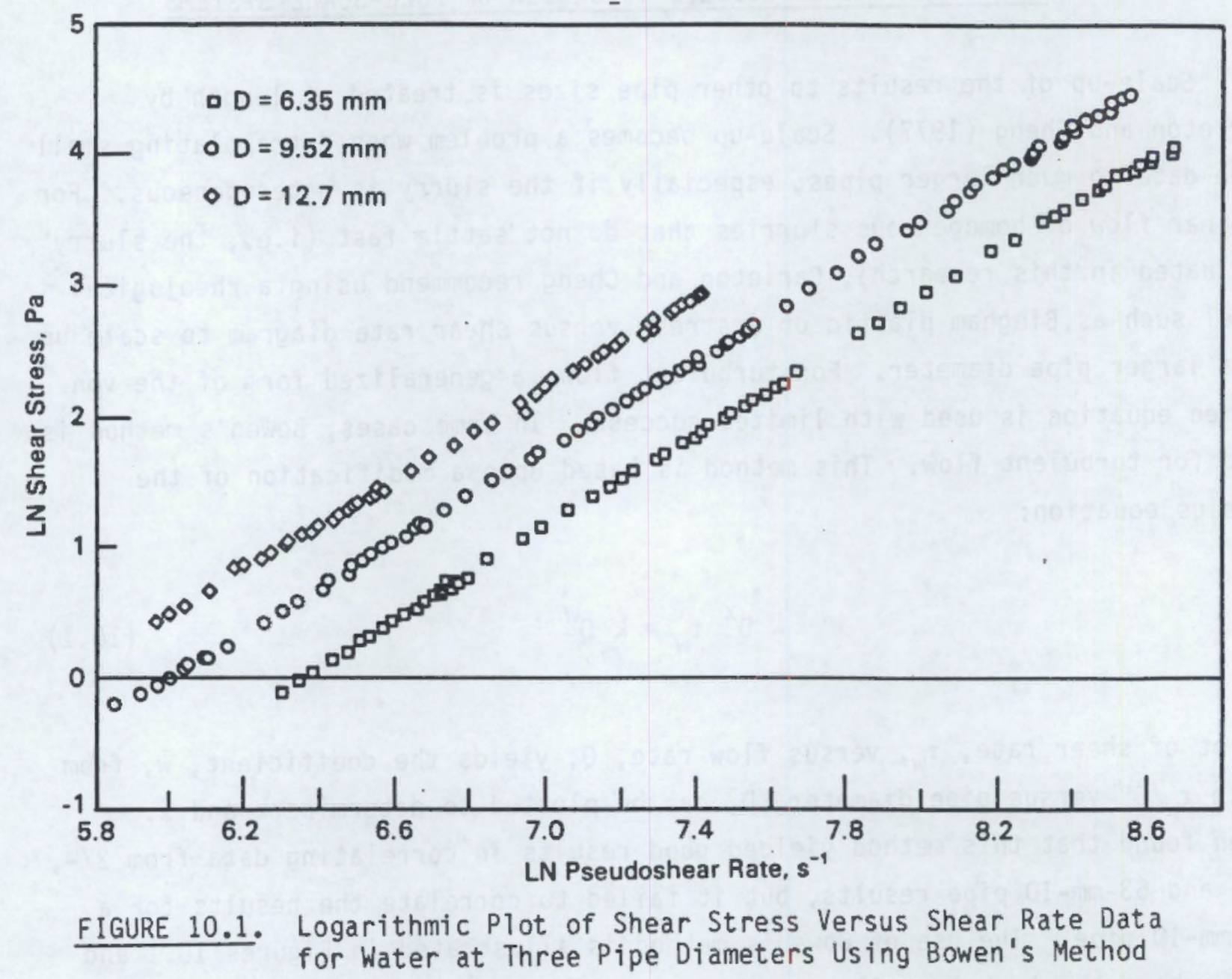

transition Reynolds numbers, a prediction that was observed in the experiments (Table 6.6 and Figure 6.18 ). Note that a pseudoplastic model would predict only one transition Reynolds number for all three pipe sizes.

The model of Hanks appears to predict the laminar and turbulent behavior as well as the transition Reynolds number fairly well (Table 6.6). Accurate rheological parameters should be used in the model, however, so a nonlinear regression routine should be used on viscometer data over as wide a range of shear rates as possible. It is certainly advisable to have the viscometer shear rates bracket those that will be encountered in the full-scale system. 


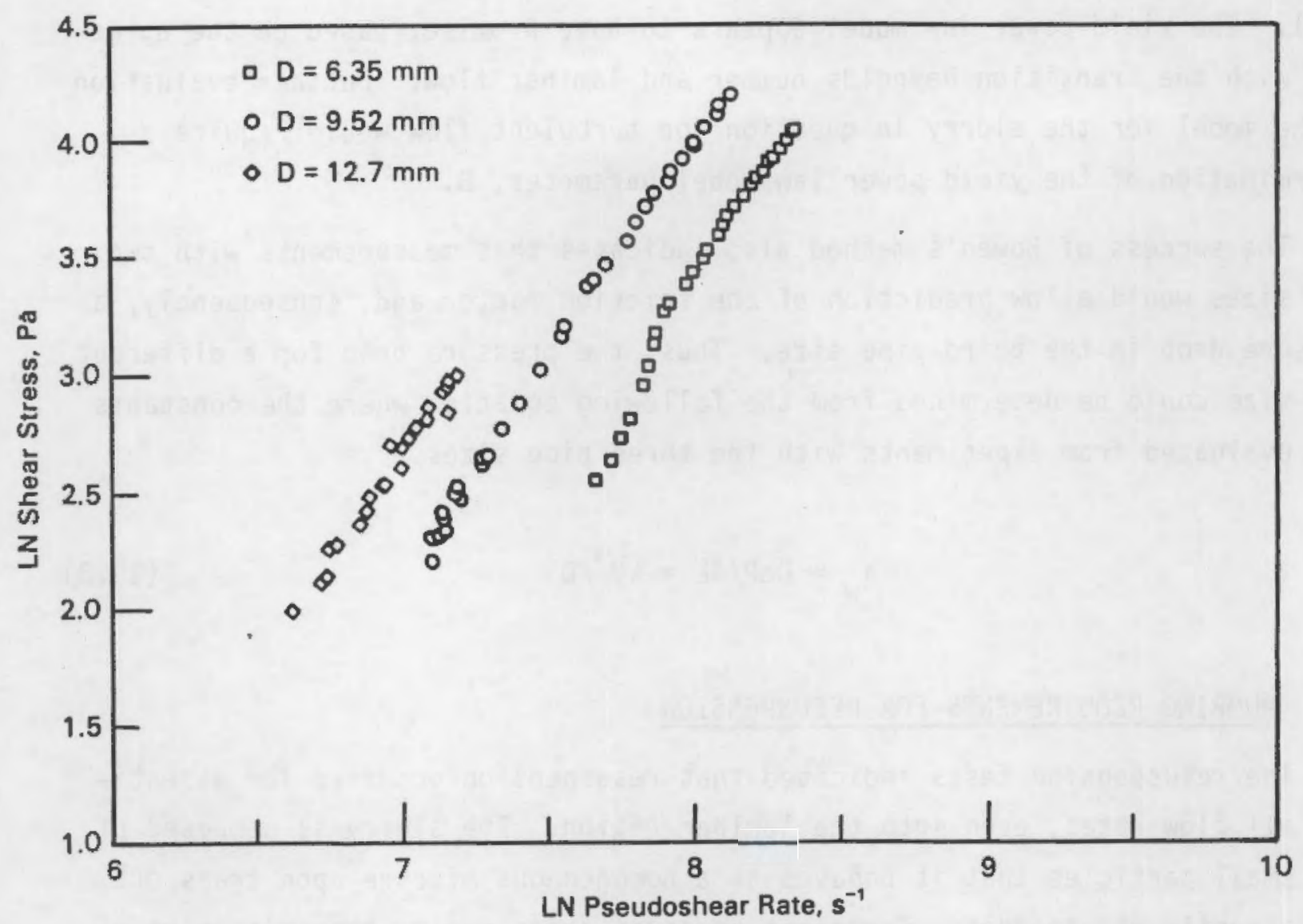

FIGURE 10.2. Logarithmic Plot of Shear Stress Versus Shear Rate Data for Slurry at Three Pipe Diameters Using Bowen's Method

\subsection{PUMPING REQUIREMENTS FOR TRANSPORT}

Pumping requirements for transport of the fully suspended slurry can be determined from the friction factor, $f$, pipe parameters, $D$ and $L$, and desired transport velocity, $V$, by means of the following formula:

$$
\Delta P=f(4 L / D)\left(\rho V^{2} / 2 g_{C}\right)
$$

The problem then is to relate the friction factor to the desired flow rate or velocity. A rheological model must be used to relate these. The accuracy of the rheological model will be determined by the agreement between the experimentally measured friction factors and the predicted ones based on the 
model. The yield power law model appears to have promise, based on the agreement with the transition Reynolds number and laminar flow. Further evaluation of the model for the slurry in question for turbulent flow would require a determination of the yield power law model parameter, 8 .

The success of Bowen's method also indicates that measurements with two pipe sizes would allow prediction of the friction factor and, consequently, a pressure drop in the third pipe size. Thus, the pressure drop for a different pipe size could be determined from the following equation where the constants were evaluated from experiments with the three pipe sizes.

$$
\tau_{W}=D \Delta P / 4 L=k v^{W} / D^{X}
$$

\subsection{PUMPING REQUIREMENTS FOR RESUSPENSION}

The resuspension tests indicated that resuspension occurred for essentially all flow rates, even into the laminar region. The slurry is composed of such small particles that it behaves as a homogeneous mixture upon transport and is easily resuspended. Transient pressure drops during the initiation of resuspension were comparable to those for initiation of fully suspended flow. The transient pressure drops were two to three times the pressure drops for fully suspended flow. The total energy required to resuspend the slurry would be proportional to the flow rate times the integral under the transient pressure curve (actual pressure less steady-state pressure). The pump horsepower, then, to get the slurry resuspended would be proportional to this energy divided by the resuspension time. Pumps should be sized, then, to convey the slurry at a given flow and have sufficient horsepower to suspend and convey the slurry. The pumps should be sized to handle transient heads of approximately three times that corresponding to steady-state flow. 


\subsection{REFERENCES}

Bird, R. B., W. S. Stewart, and E. N. Lightfoot. 1960. Transport Phenomena. John Wiley and Sons, New York.

Carleton, A. J., and D. C-H Cheng. 1974. "Design Velocities for Hydraulic Conveying of Settling Suspensions." Presented at the Third Int'1. Conf. on Hydraul. Transp. Solids in Pipes, May 15-17, Golden, Colorado, p. E5.

Carleton, A. J., and D. C-H. Cheng. 1977. "Pipeline Design for Industrial Slurries." Chemical Engineering, April 25:95-100.

Darby, R. 1984. "Determination and Utilization of Rheological Properties for Prediction of Flow Behavior of Pseudohomogeneous Slurries." Presented at the Ninth International Technical Conference on Slurry Transportation, Lake Tahoe, Nevada.

Dodge, D. H., and A. B. Metzner. 1959. "Turbulent Flow of Non-Newtonian Systems." AIChE Jour., 5(2):189.

Durand, R. 1953. "Basic Relationships of the Transportation of Solids in Pipes--Experimental Research." In Proceedings of the International Association of Hydraulic Research 5 th Congress, Minneapolis, pp. 89-103.

Gadala-Maria, F. A. 1979. "The Rheology of Concentrated Suspensions." Dissertation, Stanford University.

Hanks, R. W. 1978. "Low Reynolds Number Turbulent Pipeline Flow of Pseudohomogeneous Slurries." Presented at the Proc. 5th Int'1. Conf. Hydraul. Transp. Solids in Pipes, May 8-11, Hannover, Federal Republic of Germany, p. C2.

Hanks, R. W. 1980. "Slurry Pipeline Hydraulics: Principles, Problems and Solutions." Presented at the 1980 Meeting of the American Society of Mechanical Engineers, New Orleans.

Hanks, R. W., and D. G. Sloan. 1981. "A Rheology Based Correlation for Minimum Deposition Velocities." Presented at the Sixth Internation Technical Conference of Slurry Transportation, Las Vegas, Nevada.

Hanks, R. W. 1982. "A New Viscometer for Determining the Effect of Particle Size Distributions and Concentration upon Slurry Rheology." Presented at Seventh International Technical Conference on Slurry Transportation, Las Vegas, Nevada.

Hanks, R. W., and K. H. Hanks. 1984. "The Importance of Yield Stresses in Turbulent Slurry Pipeline Transport of Solids." Presented at the Ninth International Technical Conference of Slurry Transportation, Lake Tahoe, Nevada. 
Herschel, W. H., and R. Bulkley. 1926. "Measurement of Consistency as Applied to Rubber Benzene Solutions." In Proceedings of the ASTM, 26 (Part II):621.

Hodgman, C. D., ed. 1960. Handbook of Chemistry and Physics. Chemical Rubber Publishing Co., Cleveland, Ohio.

Jastrzebski, Z. D. 1967. "Entrance Effect and Wall Effects in an Extrusion Rheometer during the Flow of Concentrated Suspensions." I\&EC Fund., $6(3): 445$.

Kao, D. T., and D. 3. Wood. 1974. "Incipient Motion of Solids in Solid-Liquid Transport Systems." Transactions of the American Institute of Mechanical Engineers 255:39-44.

Leighton, D., and A. Acrivos. 1986. "Viscous Resuspension," Chertical Engineering Science 41(6):1377.

Mannheimer, R. J. 1985. "Flow Characteristics at Shear Stresses Near the Yield Value." In Proceedings of the Tenth International Conference on Slurry Technology. March 1985, Lake Tahoe, Nevada.

McCarthy, D., M. K. C. Chan and R. 0. Lokken. 1986. "Rheological Evaluation of Pretreated Cladding Removal Wastes." PNL-5589, Pacific Northwest Laboratory, Richland, Washington.

Mooney, M. 1931. "Explicity Formulas for \$lip and Fluidity" J. Rheol., 2:210.

Motyka, T., and C. T. Randa11. 1983. "Predicting Transport Requirements for Radioactive Waste Slurries." Presented at the Eighth International Technical Conference of Slurry Transportation, San Francisco, California.

Newitt, D. M., et. al. 1955. "Hydraulic Conveying of Solids in Horizontal Pipes." Trans. Instn. Chem. Engrs. 33:93.

0ldroyd, J. G. 1960. Rheology, John Wiley and Sons, New York, New York.

Peterson, M. E., D. McCarthy and K. D. Muhlstein. 1986. Design of a Mixing System for Simulated High-Level Nuclear Waste Melter Feed Slurries. PNL-5745, Pacific Northwest Laboratory, Richland, Washington.

Pouska, G. A., and J. M. Link. 1981. "Differential Flow Rheometer." Presented at the Proc. 6th Int'1. Hydraul. Transp. Solids in Pipes, March 24-27, Las Vegas, Nevada.

Rabinowitsch, B. Z. 1929. "Uber die Viskositat und Elastizitat von Solen." Z. Physak. Chem. A145:1.

Schlichting, H. 1968. Boundary Layer Theory. McGraw-Hill, New York.

Schramm, G. 1981. Introduction to Practical Viscometry. Gebruder HAAKE GmbH, West Germany. 
Shields, A. 1936. Application of Similarity Principles and Turbulence Research to Bed-Load Movement. No. 67, Hydrodynamics Laboratory, California Institute of Technology, Pasadena, California.

Sinclair, C. G. 1962. "The Limit Deposit-Velocity of Heterogeneous Suspensions." In Proceedings of Symposium on Interaction Between Fluids and Particles, Instn. Chem. Engrs., pp. 79-84,

Skel land, A. H. P. 1967. Non-Newtonian Flow and Heat Transfer. John Wiley and Sons, New York.

Thomas, D. G. 1961a. "Transport Characteristics of Suspensions - Part II." AlChE Jour. 7(3):423-430.

Thomas, D. G. 1961b. "Transport Characteristics of Suspensions - Part III." AIChE Jour. $7(3): 431-437$.

Thomas, D. G. 1962. "Transport Characteristics of Suspensions - Part VI." AIChE Jour. 8(3):373-378.

Thomas, D. G. 1963. "Non-Newtoninan Suspension - Part I." Ind. Eng. Chem. $55(11): 18-29$.

Thomas, D. G. 1964. "Transport Characteristics of Suspensions: Part XI; Representation of Periodic Phenomena on Flow Regime Diagram for Dilute Suspension Transport." AIChE Jour. 10(3):305.

Torrance, B. 1963. "Friction Factors for Turbulent Non-Newtonian Fluid Flow in Circular Pipes." So. Africa. Mech. Eng., 13:89-91.

Tsai, S. C., and E. H. Knell. 1986. "Viscometry and Rheology of Coal Water Slurry." Fuel 65:566.

Van Wezer, J. R., J. H. Lyons, K. Y. Kim, and R. E. Colwell. 1963. Viscometry and Flow Measurement. Interscience, John Wiley and Sons, New York.

Wasp, E. J., J. P. Kenny, and R. L. Gandhi. 1977. Solid-liquid Flow: Slurry Pipeline Transportation. Trans Tech Publications, Clausthal, Germany. 
DISTRIBUTION

No. of

Copies

OFFSITE

30 DOE Technical Information Center

6 DOE Office of Civilian

Radioactive Waste Management Forrestal Building Washington, DC 20585

ATTN: C. R. Cooley, RW-40

J. R. Hilley, RW-30

S. H. Kale, RW-20

D. E. Shelor, RW-32

R. Stein, RW-23

L. H. Barrett, RW-33

3 DOE Office of Defense Waste \& Transportation Management GTN Washington, DC 20545
ATTN:
G. H. Daly, DP-123
J. E. Lytle, DP-12
T. C. Chee

4 DOE Office of Remedial Action GTN and Waste Technology

Washington, DC 20545

ATTN: J. A. Coleman, NE-24

T. W. McIntosh, NE-24

W. R. Voigt, NE-20

H. F. Walter, NE-24

A. T. Clark

Division of Fuel Material Safety

Nuclear Regulatory Commission Washington, DC 20555

V. Stello

Office of the Executive

Director for Operations

Mail Station 6209

Nuclear Regulatory Commission

Washington, DC 20555
No. of

Copies

S. Meyers

Environmental Protection Agency Office of Radiation Programs (ANR-458)

401 M Street, S.W.

Washington, DC 20460

J. M. McGough

DOE Albuquerque Operations Office

P.0. Box 5400

Albuquerque, NM 87185

P. G. Hagan

Joint Integration office

Carlmont Executive 1

4308 Carlisle NE

Albuquerque, NM 87107

E. Maestas

DOE West Valley Operations Office

P.0. Box 191

West Valley, NY 14171

3 DOE Idaho Operations office

550 Second Street

Idaho Fal1s, ID 83401

ATTN: S. T. Hinschberger

J. P. Hamric

J. L. Lyle

F. T. Fong

DOE San Francisco Operations

1333 Broadway

Oakland, CA 94612

M. R. Jugan

DOE Oak Ridge Operations Office P.O. Box E

Oak Ridge, TN 37830 
No. of

Copies

W. J. Brumley

DOE Savannah River Operations Office

P.0. Box A

Aiken, SC 29801

M. J. Steindler

Argonne National Laboratory

9700 South Cass Avenue

Argonne, IL 60439

C. 5. Abrams

Argonne National Laboratory.

P.0. Box 2528

Idaho Falls, ID 83401

3 Battelle Memorial Institute

Project Management Division

505 King Avenue

Columbus, $\mathrm{OH} 43201$

ATTN: W. A. Carbeiner

W. S. Madia

Technical Library

L. D. Ramspott

Lawrence Livermore National Laboratory

University of California

P.0. Box 808

Livermore, CA 94550

D. T. Oakley, MS 671

Los Al amos Scientific Laboratory

P.0. Box 1663

Los Alamos, NM 87544

5 Oak Ridge National Laboratory

P.0. Box Y

Oak Ridge, TN 37830

ATTN: J. 0. Blomeke

W. D. Burch

R. T. Jubin

L. J. Mezga

D. H. Turner
No. of

Copies

Sandia Laboratories

P.0. Box 5800

Albuquerque, NM 87185

ATTN: Technical Library

J. R. Berreth

Westinghouse Idaho Nuclear Co., Inc.

P.0. Box 4000

Idaho.Falls, ID 83401

6 E. I. du Pont de Nemours Company

Savannah River Laboratory

Aiken, SC 29801

ATTN: M. D. Boersma

J. G. Glasscock

R. G. Baxter

J. R. Knight

M. J. Piodinec

C. T. Randa 11

E. A. Jennrich

EG\&G Idaho

P.0. Box 1625

Idaho Falls, ID 83415

R. Shaw

Electric Power Research Institute

3412 Hillview Avenue

P.0. Box 10412

Palo Alto, CA 94304

5 West Valley Nuclear Services

Company

P.0. Box 191

West Valley, NY 14171

ATTN: S. M. Barnes

C. C. Chapman

J. E. Krauss

S. J. Marchette

J. M. Pope 
No. of

Copies

J. L. White

Energy Research \& Development Authority

Empire State Plaza

Albany, NY 12223

A. K. Postma

Rt. 1, Box 46A

Halfway, OR 97834

4 University of Idaho

Department of Chemical

Engineering

Moscow, ID 83843

ATTN: T. E. Carleson (2)

D. C. Drown

R. E. Hart

ONSITE

6 DOE Richiand Operations Office

E. A. Bracken

G. J. Bracken

C. E. Collantes

C. R. DeLannoy

M. W. Shupe

J. J. Sutey

15 Westinghouse Hanford Company

A. J. DiLiberto

J. M. Henderson

0 . L. Krueger

R. E. Lerch

D. E. McKenney

J. L. Scott

G. E. Stegen

L. C. Stegen

M. C. Teats

D. A. Turner

D. D. Wodrich

J. D. Watrous

R. D. Wojtasek

B. D. Wolfe

J. C. Womack
No. of

Copies

47 Pacific Northwest Laboratory

C. R. Alten

W. W. Ballard, Jr.

W. F. Bonner

R. A. Brouns (2)

H. C. Burkholder

J. R. Carrell

C. L. Fow

T. L. Gitbride

D. K. Hilliard

L. K. Holton

W. L. Kuhn

L. T. Lakey

D. E. Larson

J. L. MCE] roy

R. K. Nakaoka

J. M. Perez, Jr.

R. D. Peters

M. E. Peterson (15)

C. M. Ruecker

P. A. Scott

S. C. Slate

J. E. Surma

G. T. Thornton

J. H. Westsik, Jr.

G. A. Whyatt

Publishing Coordination (2)

Technical Report Files (5) 
$:$ 